\title{
Gamma Spectrometry Examination of the AGR-3/4 Irradiation
}

October 2021

\author{
Jason M. Harp \\ John D. Stempien \\ Paul A. Demkowicz
}

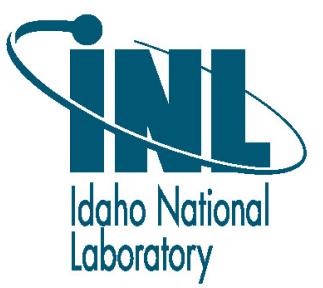




\section{DISCLAIMER}

This information was prepared as an account of work sponsored by an agency of the U.S. Government. Neither the U.S. Government nor any agency thereof, nor any of their employees, makes any warranty, expressed or implied, or assumes any legal liability or responsibility for the accuracy, completeness, or usefulness, of any information, apparatus, product, or process disclosed, or represents that its use would not infringe privately owned rights. References herein to any specific commercial product, process, or service by trade name, trade mark, manufacturer, or otherwise, does not necessarily constitute or imply its endorsement, recommendation, or favoring by the U.S. Government or any agency thereof. The views and opinions of authors expressed herein do not necessarily state or reflect those of the U.S. Government or any agency thereof. 
INL/EXT-20-58254

Revision 1

\title{
Gamma Spectrometry Examination of the AGR-3/4 Irradiation
}

\author{
Jason M. Harp \\ John D. Stempien \\ Paul A. Demkowicz
}

October 2021

\begin{abstract}
Idaho National Laboratory Advanced Reactor Technologies

Idaho Falls, Idaho 83415
\end{abstract}

http://www.art.inl.gov

Prepared for the

U.S. Department of Energy

Office of Nuclear Energy

Under DOE Idaho Operations Office

Contract DE-AC07-05ID14517 



\title{
Gamma Spectrometry Examination of the AGR-3/4 Irradiation
}

\author{
INL/EXT-20-58254
}

Revision 1

October 2021

Technical Reviewer:

$\frac{\text { Ryan of Fronte }}{\text { Rgan Frotk }}$

Gamma Spectroscopist

Approved by:

Travis Mitchell

$10 / 25 / 2021$

Travis Mitchell

Date

ART Program Manager

Michollo sharp

Michelle T. Sharp

\begin{tabular}{c}
$10 / 25 / 2021$ \\
\hline Date \\
$10 / 25 / 2021$ \\
\hline Date
\end{tabular}

ART Quality Engineer 


\section{SUMMARY}

The results from gamma spectrometry of the different components from the third U.S. Advanced Gas Reactor (AGR) tristructural isotropic (TRISO)-coated particle fuel irradiation test (AGR-3/4) have been analyzed. This experiment was designed to provide information about in-pile fission product migration. In each of the 12 capsules, a single stack of four compacts contained roughly 1898 TRISO particles and 20 designed-to-fail (DTF) particles per compact. The stack of compacts was surrounded by inner and outer graphite and/or graphitic matrix rings and a graphite sink ring. These items were irradiated in the Advanced Test Reactor (ATR) at Idaho National Laboratory (INL). Gamma spectrometry has been used to evaluate the gamma-emitting fission product inventory of compacts from the irradiation and evaluate the burnup of these compacts, based on the activity of the radioactive cesium isotopes (i.e., Cs-134 and Cs-137) in the compacts. The burnup from gamma spectrometry compares well with the predicted burnup from simulations.

The inner and outer rings were also examined by gamma spectrometry to evaluate the total fission product inventory of the rings, and gamma emission computed tomography (GECT) was used to investigate the spatial distribution of gamma-emitting fission products within the rings. This report focuses on results obtained via the Precision Gamma Scanner (PGS) for the compacts and inner and outer rings. These nondestructive gamma measurements are currently being compared to recent destructive measurements of spatial fission product distributions in the rings and to predictions made via mathematical modeling. A separate report discusses the entire AGR-3/4 fission product mass balance for all irradiation capsule components, including the inner and outer rings, graphite sink rings, capsule spacers, capsule foils, through tubes, and felts.

This report is similar to a paper presented at the HTR2016 conference (Harp et al. 2016), but it has been updated to reflect all the data that has been collected on AGR-3/4 using PGS. The Appendices contain extensive data from the collected gamma spectra. 


\section{CONTENTS}

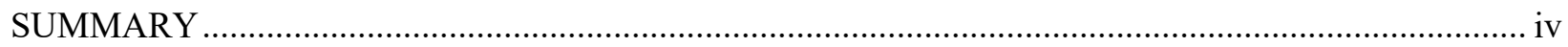

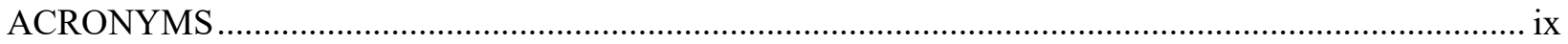

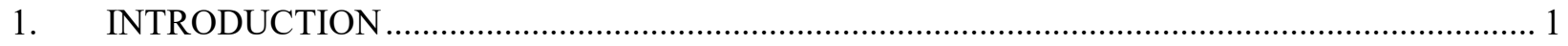

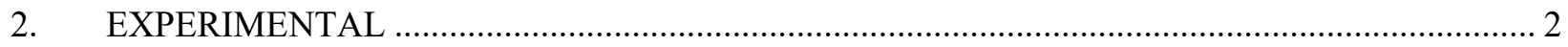

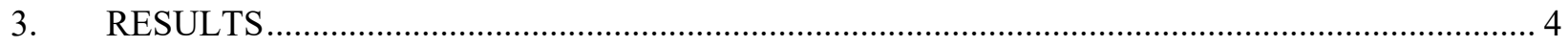

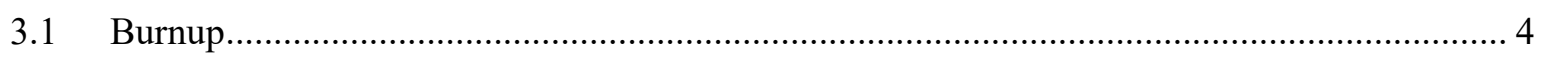

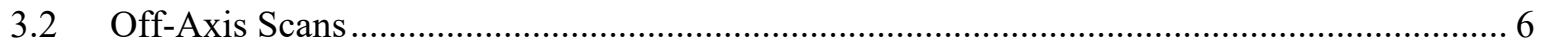

3.3 Gamma Emission Computed Tomography ..................................................................... 9

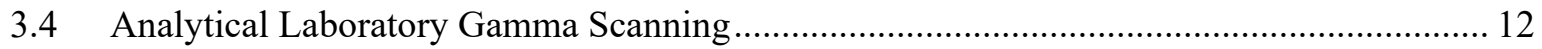

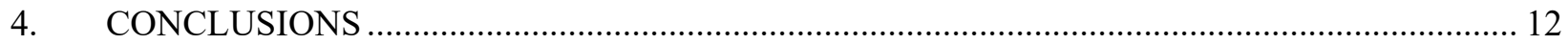

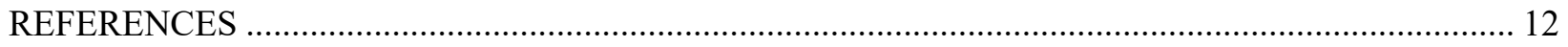

Appendix A Burnup Conversion and Total Compact Activity for Selected Isotopes ............................. 15

Appendix B Measured Versus Calculated Burnup for Each Compact …............................................. 18

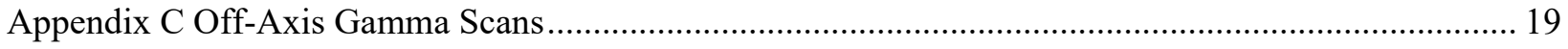

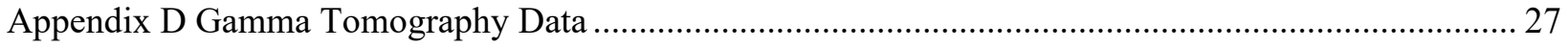

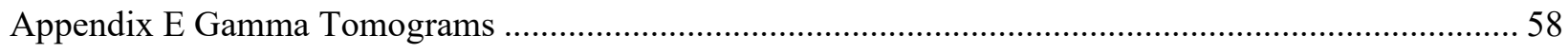

\section{FIGURES}

Figure 1. Major components of a representative AGR-3/4 standard capsule. ......................................... 2

Figure 2. Illustration of the PGS system (left) and the PGS collimator (right) ........................................ 4

Figure 3. Illustration of off-axis scans of inner (left) and outer (right) rings from AGR-3/4 ................... 4

Figure 4. Burnup evaluation of AGR-3/4 based on gamma spectrometry of AGR-3/4 fuel

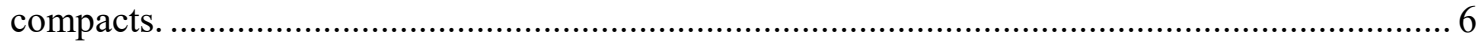

Figure 5. Off-axis scans of the Capsule 3 inner ring (left) and outer ring (right)................................... 8

Figure 6. Off-axis scans of the Capsule 8 inner ring (left) and outer ring (right) ................................. 8

Figure 7. Co-60 tomogram for IR-07 taken at the axial mid-plane of the ring....................................... 11

Figure 8. Cs-134 tomogram for the upper level scans from IR-03....................................................... 11

Figure 9. Ag-110m tomogram for the upper level scan from IR-03 .................................................. 11

Figure 10. Eu-154 tomogram for the IR-07 taken at the axial mid-plane of the ring ............................. 11

Figure 11. Cs-137 tomogram for the upper level scan from IR-10 ...................................................... 11 
Figure 12. Ag-110m tomogram for the mid-plane scan of OR-08

\section{TABLES}

Table 1. List of AGR-3/4 capsules noting capsule type, material for the inner and outer ring, and INL drawing number. 2

Table 2. Total decay-corrected activity measured in AGR-3/4 inner rings. Uncertainties in

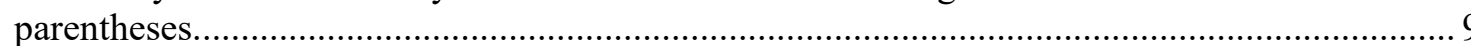

Table 3. Total decay-corrected activity measured in AGR-3/4 outer rings. Uncertainties in

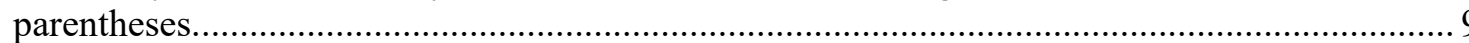




\section{ACRONYMS}

AGR Advanced Gas Reactor

ATR Advanced Test Reactor

DTF designed-to-fail

FIMA fissions per initial heavy-metal atom

GECT gamma emission computed tomography

HFEF Hot Fuel Examination Facility

HPGe high-purity germanium

INL Idaho National Laboratory

IR inner ring

$\mathrm{M} / \mathrm{C}$ measured-to-calculated ratio

OR outer ring

PGS Precision Gamma Scanner

PIE post-irradiation examination

TRISO tristructural isotropic 


\section{Gamma Spectrometry Examination of the AGR-3/4 Irradiation}

\section{INTRODUCTION}

The AGR-3/4 irradiation was the third test in a series of fuel irradiations under the Advanced Gas Reactor (AGR) program, which is qualifying $\mathrm{UCO}$ (a heterogeneous mixture of $\mathrm{UO}_{2}$ and uranium carbide) tristructural isotropic (TRISO) fuel for use in high-temperature reactors. The first irradiation, AGR-1, and its associated post-irradiation examination (PIE), demonstrated the performance of TRISO fuel produced on a laboratory scale (Demkowicz et al. 2016; Morris et al. 2016). The AGR-2 irradiation and its associated PIE were designed to demonstrate the performance of TRISO particles produced on a larger, engineering scale (Demkowicz 2019; Petti et al. 2017). The AGR-3/4 test was performed in the Idaho National Laboratory (INL) Advanced Test Reactor (ATR) for 10 cycles or 369 effective full power days, and fuel compact-average burnups ranged from 4.85 to $15.27 \%$ fissions per initial heavy-metal atom (FIMA). The AGR-3/4 irradiation was designed to provide insight on in-pile fission product behavior and transport.

Gamma spectrometry was used previously in evaluating the burnup of the AGR-1 TRISO fuel compacts (Harp et al. 2014) as well as the inventory of Ag-110m remaining in the AGR-1 compacts (Demkowicz et al. 2013). Additionally, gamma spectrometry of the AGR-1 graphite fuel compact holders was used to identify compacts containing defective SiC TRISO particles (Hunn et al. 2016). Gamma spectrometry was also utilized to measure the total inventory of gamma-emitting fission products outside of the fuel compacts in each of the capsules in AGR-1 (Demkowicz et al. 2013). The same techniques and processes have been applied to the similar compacts and capsule components in AGR-2 (Harp et al. 2020; Stempien and Demkowicz 2020) and to the unique fuel and other test articles from AGR-3/4.

Major components of the AGR-3/4 capsules are shown in Figure 1 and include four fuel compacts stacked at the center of each capsule, an inner ring of graphite (PCEA or IG-110) or graphitic matrix material, an outer ring made of graphite (PCEA or IG-110), a sink ring made of PCEA graphite, through tubes, and the stainless-steel capsule shell. The test train contained 12 capsules of four fuel compacts each, and each capsule was independently controlled for temperature and monitored for release of fission gases (Collin 2015; Collin et al. 2018). Capsules were numbered from the bottom (Capsule 1) to the top (Capsule 12) of the test train, and the compacts were also numbered within each capsule from the bottom to the top. In addition to the "standard" AGR-3/4 capsules depicted in Figure 1, some capsules were "fuel-body" capsules, where the outer ring had a sealed graphite floor and lid. Of the fuel-body-type capsules, only Capsule 4 was disassembled and its contents examined by gamma spectrometry. The configuration of each AGR-3/4 capsule is listed in Table 1.

To provide a source of fission products whose migration through the compacts and other capsule components could be measured, 20 designed-to-fail (DTF) particles were aligned down the center of the cylindrical fuel compacts amid approximately 1898 TRISO-coated driver fuel particles per compact (Hunn et al. 2011). DTF particles consisted of UCO fuel kernels coated in a $20 \mu \mathrm{m}$-thick layer of highly anisotropic pyrocarbon. A summary of kernel, DTF particle, and TRISO-coated driver particle properties, as well as references to fuel characterization reports, can be found in Collin (2015).

This report focuses on the processes and results of gamma scanning the AGR-3/4 fuel compacts and the concentric rings of graphite and/or graphitic matrix material that surrounded the fuel compacts during irradiation. These results have been used as a component of the overall AGR-3/4 irradiation experiment fission product mass balance, which quantified the fraction of fission products that escaped the fuel compacts during irradiation (Stempien et al. 2018a). 


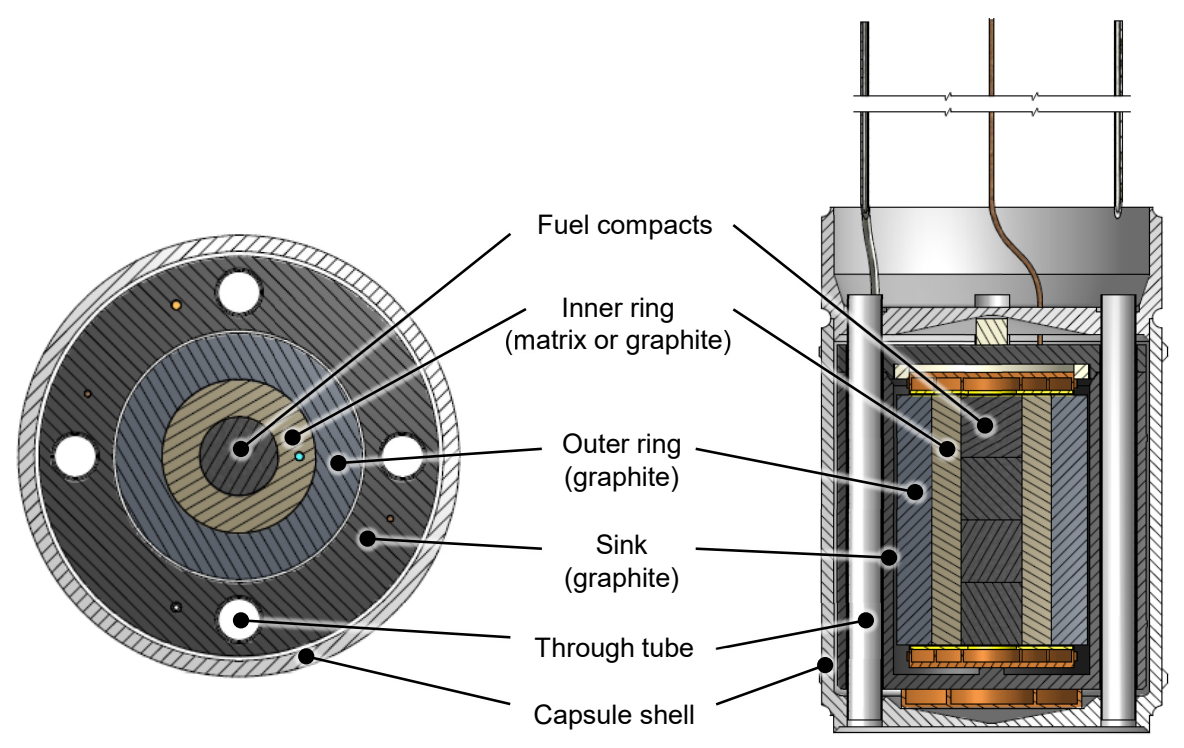

Figure 1. Major components of a representative AGR-3/4 standard capsule.

Table 1. List of AGR-3/4 capsules, noting capsule type, material for the inner and outer rings, and INL drawing number.

\begin{tabular}{|c|c|c|c|c|c|c|c|}
\hline \multirow{2}{*}{ Capsule } & \multirow{2}{*}{ Type } & \multicolumn{3}{|c|}{ Material } & \multicolumn{2}{c|}{ Thickness (mm) } & \multirow{2}{*}{ Drawing ID } \\
\cline { 3 - 7 } & & $\begin{array}{c}\text { Inner } \\
\text { Ring }\end{array}$ & $\begin{array}{c}\text { Outer } \\
\text { Ring }\end{array}$ & $\begin{array}{c}\text { Sink } \\
\text { Ring }\end{array}$ & $\begin{array}{c}\text { Inner } \\
\text { Ring }\end{array}$ & $\begin{array}{c}\text { Outer } \\
\text { Ring }\end{array}$ & \\
\hline 12 & Standard & Matrix & PCEA & PCEA & 5.96 & 5.22 & DWG-602712 \\
\hline 11 & Fuel body & Matrix & PCEA & PCEA & 5.02 & 4.50 & DWG-602711 \\
\hline 10 & Standard & PCEA & PCEA & PCEA & 5.96 & 6.73 & DWG-602710 \\
\hline 9 & Fuel body & Matrix & IG-110 & PCEA & 5.96 & 7.49 & DWG-602709 \\
\hline 8 & Standard & IG-110 & IG-110 & PCEA & 5.96 & 7.23 & DWG-602708 \\
\hline 7 & Standard & Matrix & PCEA & PCEA & 5.96 & 6.72 & DWG-602707 \\
\hline 6 & Fuel body & Matrix & PCEA & PCEA & 5.96 & 7.56 & DWG-602706 \\
\hline 5 & Standard & Matrix & PCEA & PCEA & 5.96 & 7.56 & DWG-602705 \\
\hline 4 & Fuel body & Matrix & PCEA & PCEA & 5.96 & 7.49 & DWG-602704 \\
\hline 3 & Standard & PCEA & PCEA & PCEA & 5.96 & 4.50 & DWG-602703 \\
\hline 2 & Fuel body & Matrix & PCEA & PCEA & 5.96 & 6.22 & DWG-602702 \\
\hline 1 & Standard & Matrix & PCEA & PCEA & 5.65 & 4.51 & DWG-602701 \\
\hline
\end{tabular}

${ }^{a}$ DWG-602716

\section{EXPERIMENTAL}

Gamma scanning was accomplished using the INL Hot Fuel Examination Facility (HFEF) Precision Gamma Scanner (PGS). PGS has three major components: the detector system, collimator, and stage. The detector system consists of a digital multichannel analyzer and a high-purity germanium (HPGe) detector that is surrounded by a Compton suppression detector. The collimator is approximately $2.13 \mathrm{~m}$ long with an aperture that has a fixed width of $2.22 \mathrm{~cm}$ and a variable height of 0.254 to $0.00254 \mathrm{~cm}$. The collimator can also be rotated $90^{\circ}$ so that the fixed width of the aperture is vertical instead of horizontal. The stage suspends items before the collimator and moves them in a plane parallel to the face of the collimator. The stage can also rotate items about a central axis. PGS and its collimator are illustrated in Figure 2.

As in AGR-1 and AGR-2 PIE, AGR-3/4 compacts were individually encapsulated in aluminum containers, and these containers were stacked inside a thin-walled aluminum tube that was suspended in front of the PGS collimator. Eu-152 calibration sources were packaged in equivalent containers and 
placed at the bottom and top of the same aluminum tube containing the compacts. These Eu-152 sources were scanned before the first compact and after the last compact to confirm the performance of the system. Each compact was typically scanned in $0.254 \mathrm{~cm}$ steps, for a live time of 60 minutes per step.

Burnup was determined from the measured Cs-134 to Cs-137 activity ratio, using the technique discussed in (Harp et al. 2014). Using coefficients derived from simulations specific to the AGR-3/4 experiment, the measured burnup can be expressed as a linear function of the measured Cs-134:Cs-137 activity ratio. These coefficients are given in Table A.1 in Appendix A. Similarly, the burnup can be determined from the total Cs-137 activity measured in each compact. The coefficients used to convert Cs137 activity to burnup are also given in Table A.1. The burnup determined by the ratio of Cs-134 to Cs137 can either be determined over the entire compact based on total activities, or locally, for every spectrum collected from each compact. This local measurement of burnup reveals the burnup gradients present in some compacts. The measured burnups were compared to the predicted burnups from (Sterbentz 2015).

The collected spectra were analyzed to evaluate both the gamma-emitting fission product content and burnup of each compact. Measured fission product inventories can be compared to the predicted fission product inventories from (Sterbentz 2015). Several different gamma-emitting fission products were quantified, including Zr-95, Ru-106, Ag-110m, Cs-134, Cs-137, Ce-144, and Eu-154.

In addition to the compacts, the inner and outer rings were also gamma scanned in PGS. Inner and outer rings are referred to by IR or OR and their two-digit capsule number (e.g., IR-07 for the Capsule 7 inner ring). The goal of these scans was to estimate the inventory and spatial distribution of fission products in the rings. The distribution of different fission products can be used to infer their transport behavior during irradiation. The ultimate goal is to use the distribution of fission products in the rings to compare with transport models of the AGR-3/4 experiment (Humrickhouse et al. 2016), to compare to destructive ring analyses (Stempien et al. 2018b), and to improve the understanding of fission product transport in fuel matrix and core graphite materials.

Inner and outer rings have been scanned in two ways. The rings were loaded into thin-walled Al fixtures that fix the ring geometry to the coordinate system of the PGS in a known and repeatable way (see INL Drawings 782064, 782067, and 782068). Then, they were scanned in two off-axis sweeps to identify axial levels of interest and to estimate the total activity of different isotopes in the rings. The offaxis scans are illustrated in Figure 3 for both inner and outer rings. In these scans, half the ring is scanned in one axial sweep followed by the other half in a second axial sweep. If an axial level of interest is identified, a tomographic scan of that level is performed, during which the PGS collimator is rotated to a vertical orientation and the ring is scanned horizontally at several different angles. The scans from multiple angles (up to 16) are used to reconstruct a 2D map of the activity intensity of a particular isotope in the ring. Note that due to the geometry of the collimator opening, these maps provide the average activity intensity over a $2.22 \mathrm{~cm}$ cross-section of the ring. Increasing the number of angles will improve the spatial resolution of the generated map, but the time required to collect spectra limits the number of angles that can be collected. The collimator opening and step size can be reduced to improve spatial resolution, but reducing the collimator opening size reduces the signal, increasing the required count time to collect gamma spectra and the number of spectra that must be collected.

The general process of producing these maps is often called single photon emission computed tomography (Wernick and Aarsvold 2004). However, the AGR program and some other researchers in the nuclear fuels field have utilized this process and referred to it as gamma emission computed tomography (GECT) (Harp and Demkowicz 2014; Harp and Chichester 2016; Dobrin et al. 1997a) or some variation of that name (Barnes et al. 1982; Dobrin et al. 1997b; Caruso and Jatuff 2014). GECT maps can be constructed from gamma scanning data using different mathematical methods. Fission product activity intensity maps for the AGR-3/4 rings shown in this report were produced using the analytic method of filtered backprojection. An iterative numerical method has also been implemented by (Humrickhouse et al. 2018) that resolves some of the artifacts of the filtered backprojection method discussed in Section 3.4. 

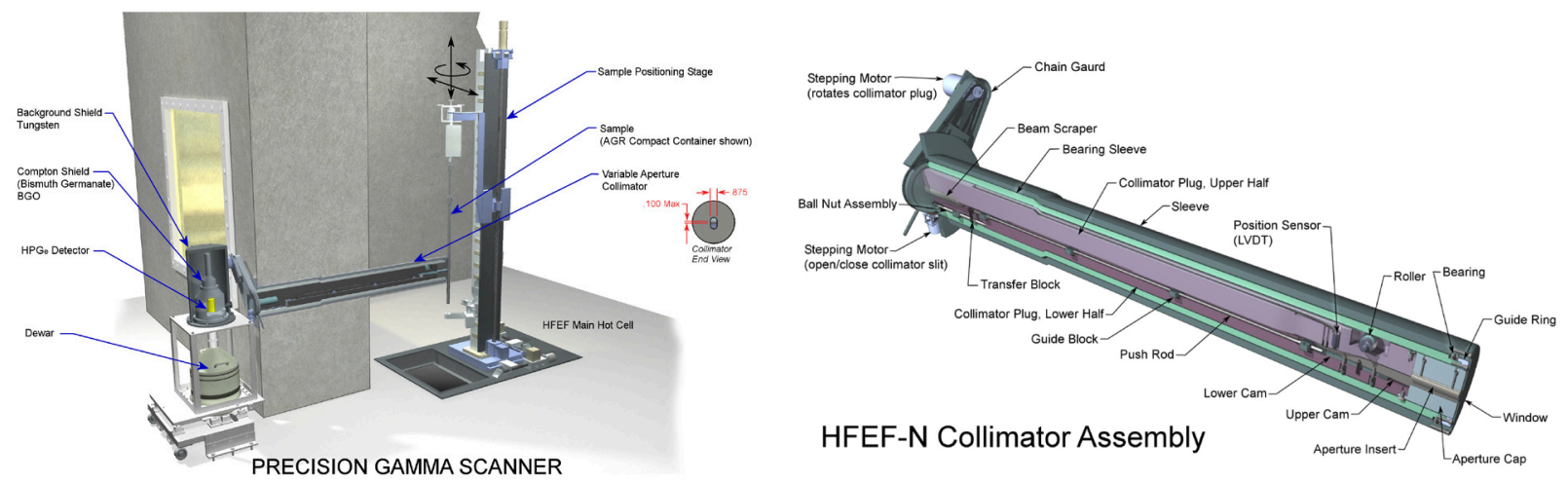

Figure 2. Illustration of the PGS system (left) and the PGS collimator (right).

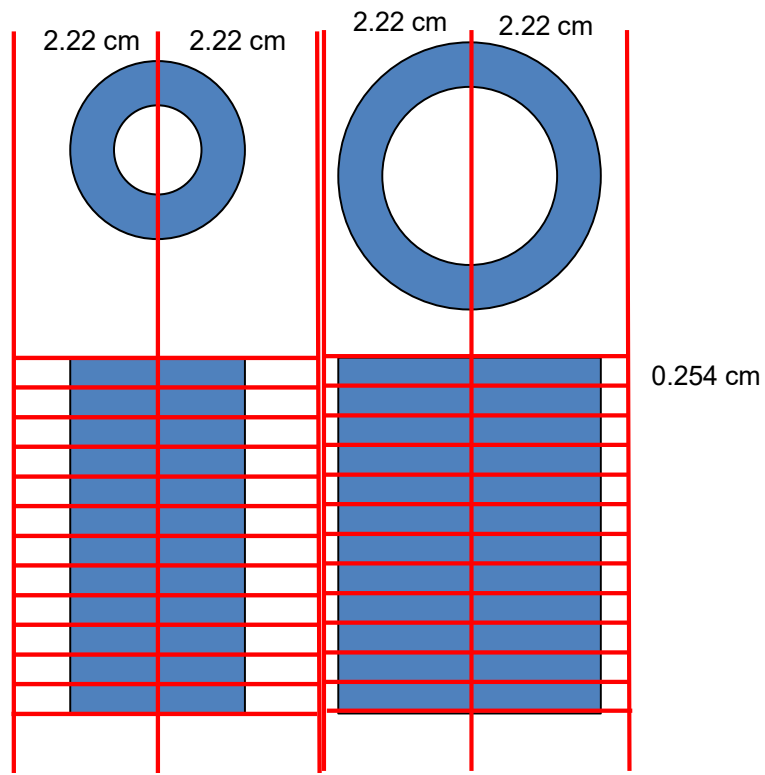

Figure 3. Illustration of off-axis scans of the inner (left) and outer (right) rings from AGR-3/4.

Work continues on collecting and analyzing data from the destructive analysis of AGR-3/4 rings (Stempien et al. 2018b). Destructive sampling has been performed on the inner and outer rings from Capsules 3, 5, 7, 8, 10, and 12 to determine the circumferentially averaged radial fission product profiles within the rings. Each ring was filled with epoxy to stabilize the ring and mounted on a rotating chuck. An end-mill (with a 1-cm wide bit) removed material from the ring in 10 to 15 steps (depending on the thickness of the ring), and a cyclone separator collected the fines from each radial segment of the ring. Most of the rings were sampled at one of their axial ends (either top or bottom) and in the axial center. All samples were sent to the Pacific Northwest National Laboratory for gamma counting and leach-burnleach analysis. These fission product profiles from destructive sampling are being compared to profiles constructed from GECT.

\section{RESULTS}

\subsection{Fuel Compact Gamma-Emitting Fission Product Inventories}

The total measured activities for Zr-95, Ru-106, Ag-110m, Cs-134, Cs-137, Ce-144, and Eu-154 are given for each compact in Table A.2 in Appendix A. These have been referenced to the end of the AGR3/4 irradiation plus one day (04/13/2014 5:00 AM Mountain Time). These activities can be compared to the predicted inventories calculated from physics simulations (Sterbentz 2015). 


\subsection{Burnup}

Data from the gamma spectrometry of compacts have been processed into burnup estimates and compared to the predicted burnup from physics calculations (Sterbentz 2015). The Cs-137 inventories of compacts are underpredicted compared to measurement, and the Cs-134 inventories are overpredicted compared to measurement. Burnup from the relative Cs-134:Cs-137 ratio is compared to simulations in Figure 4, where the burnup is plotted versus the compacts in-core position relative to core midplane $(\mathrm{x}=$ 0 ). These results agree reasonably well with the simulations but not as well as AGR-1 (Harp et al. 2014) and AGR-2 (Harp et al. 2020). The derived burnup for each compact is tabulated in Appendix B. The Capsule 4 "fuel body" was disassembled and the compacts counted; however, the other fuel-body capsules (Capsules 2, 6, 9, and 11) were not disassembled and their compacts were not counted.

The burnups determined from the total Cs-137 activity in each compact are also plotted in Figure 4. There is more scatter in these data than in the burnups from the Cs-134:Cs-137 method. The burnup evaluated using the Cs-137 method for Compacts 3-4, 4-1, 5-1 and 5-4 (x-axis positions in Figure 4: $33.65,-27.31,-17.14$, and -13.34) seems to lie outside the overall burnup trends established by the other Cs-137-based points and the Cs-134:Cs-137-based points. ${ }^{a}$ Since the Cs-137 method requires an accurate measurement of the total Cs-137 activity in the compact, it is subject to biases if portions of the sample are lost. Compacts 3-4, 4-1, and 5-1 were damaged during removal from their inner rings, and substantial pieces were missing from these compacts (Stempien et al. 2016). This explains why those data points return lower burnups and depart from the trends established by the other data points; however, it is not clear why the burnup from Cs-137 for Compact 5-4 is also a bit low. Generally, even for intact compacts, the burnups derived from the Cs-134:Cs-137 are favored over the burnups derived solely from Cs-137, because the ratios are less susceptible to the biases introduced when determining the absolute efficiency of the detector system.

\footnotetext{
${ }^{a}$ AGR-3/4 compacts were given identifiers where the first digit is the capsule and the second digit is the level of the compact in the stack of four compacts in a given capsule. Thus, Compact 3-4 is the topmost compact from Capsule 3.
} 


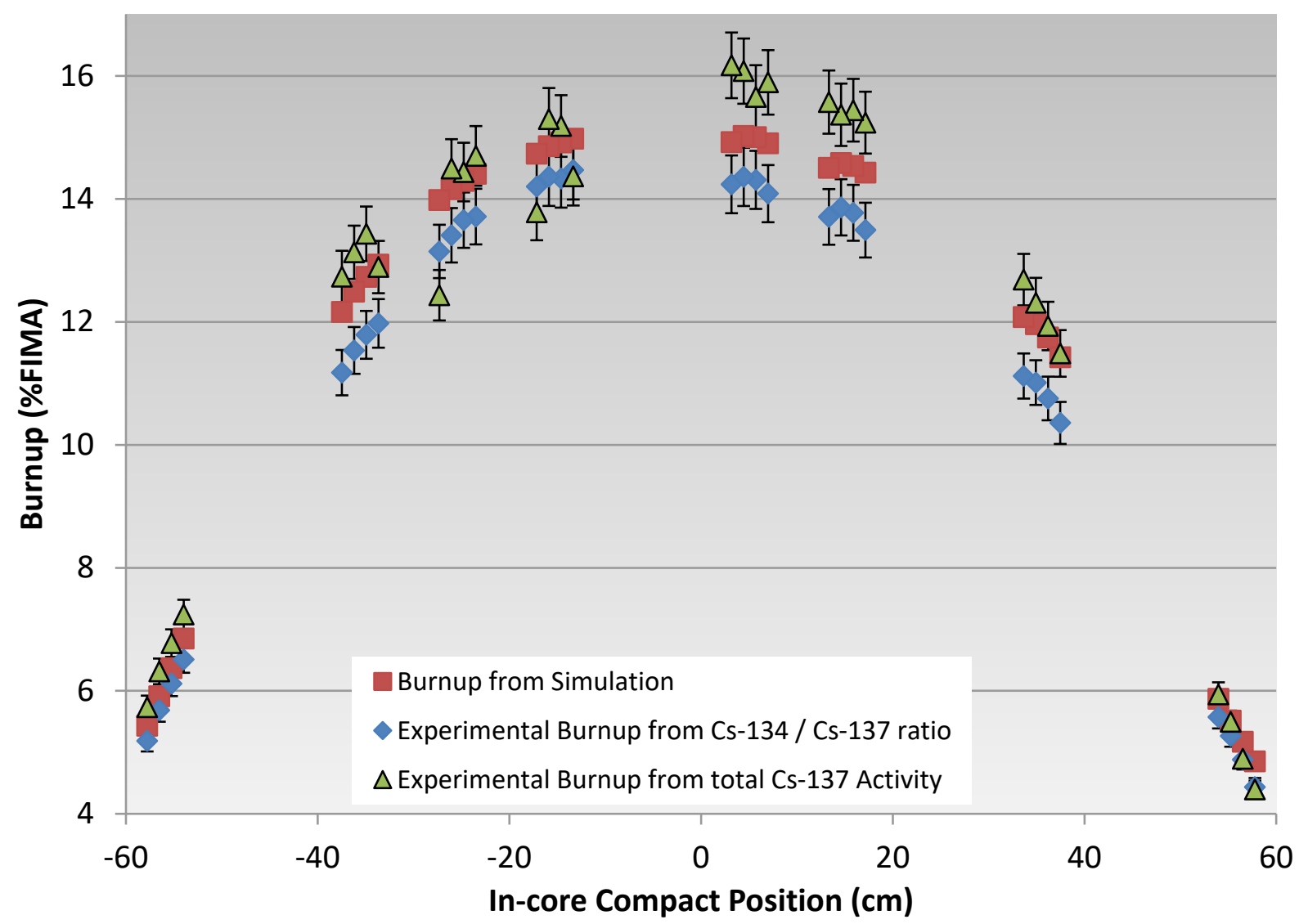

Figure 4. Burnup evaluation of AGR-3/4 based on gamma spectrometry of AGR-3/4 fuel compacts.

\subsection{Rings Off-Axis Scans}

The pair of off-axis (axial) scans for several different fission products observed in the inner and outer ring of Capsule 3 are shown in Figure 5, and similar scans are shown for Capsule 8 in Figure 6 . The scans are plotted so that the left side of the ring is shown on the left side of the plot, and the right side of the ring follows on the right side of the plot. Note that in the right plot in Figure 5, the Ag- $110 \mathrm{~m}$ values are plotted on a secondary vertical axis. In the other plots in Figure 5 and Figure 6, the Ag-110m activities were similar in magnitude to those for the other isotopes, and in those cases, all isotope activities are plotted on the same vertical axis. The scanning pattern was explained in detail in Section 2, see Figure 3. Each point in the figures represents the activity of a particular isotope in a particular location in the ring. The orientation of the rings in relationship to the azimuthal position in the reactor was not retained. Several different factors influence the fission product migration in these rings, including temperature, fission product production and release from the DTF particles (Collin et al. 2018), and type of ring material (e.g., inner rings made of IG-110 or PCEA or graphitic matrix material and outer rings made of PCEA or IG-110 graphite). The activation product, Co-60, is detectable in the Capsule 3 scans. The Co60 spike in the IR-03 plot is from an encapsulated melt wire placed in this ring that could not be removed during capsule disassembly. Both Cs-134 and Cs-137 isotopes have similar distributions in the scans, as expected. A compilation of all the off-axis scans is in Appendix C.

The AGR-3/4 capsules were specifically designed to minimize axial and azimuthal temperature gradients, such that transport of fission products would be primarily in the radial direction, simplifying the computational modeling of transport phenomena. These design characteristics include the single stack of compacts in the capsule center and thermal insulation at the ends of the capsules. However, the gamma 
spectrometry data indicate that fission product distributions exhibited some unexpected axial variations. This includes the different axial profiles of some fission products among the different rings. For example, the axial Cs profiles are peaked in the capsule center in some cases but peaked at the ends in others. These profiles likely indicate nonuniform fission product migration in the rings, possibly due to axial temperature variations or transport in the gas gaps between the compacts and the rings. This increases the complexity of the fission product transport analysis in the AGR-3/4 capsule significantly. Similarly, the Ag-110m axial distribution in the rings is also nonuniform. The significant peaking of the Ag- $110 \mathrm{~m}$ at the axial ends of the rings was unexpected and may be an indication of a non-radial migration pathway.

Table 2 and Table 3 give the total activities of isotopes measured in the inner and outer rings, respectively. The totals in the tables were created by summing the measured activity from each scan of the ring. Activities have been decay-corrected to the end of the AGR-3/4 irradiation plus one day (4/13/2014 5:00 AM MDT). Even before advanced gamma scanning techniques are applied (i.e., GECT), these data are useful to modelers as a first check on the fission product migration models (Humrickhouse et al. 2018). Note that the magnitude of Ag-110m can vary significantly, even among inner and outer rings from the same capsule.

The uncertainties and less-than values in these tables are based on counting statistics and the minimum detectable activity in each scan. The uncertainty may be asymmetrical. The lower uncertainty band is equal to the square root of the sum of the squares of the activity uncertainty for each scan with a detectable amount of a given isotope. The upper uncertainty band is also equal to the square root of the sum of the squares of the activity uncertainty for each scan with a detectable amount of a given isotope, but, in the case of a scan with an undetectable amount of a given isotope, the activity uncertainty is replaced with the minimum detectable activity. 

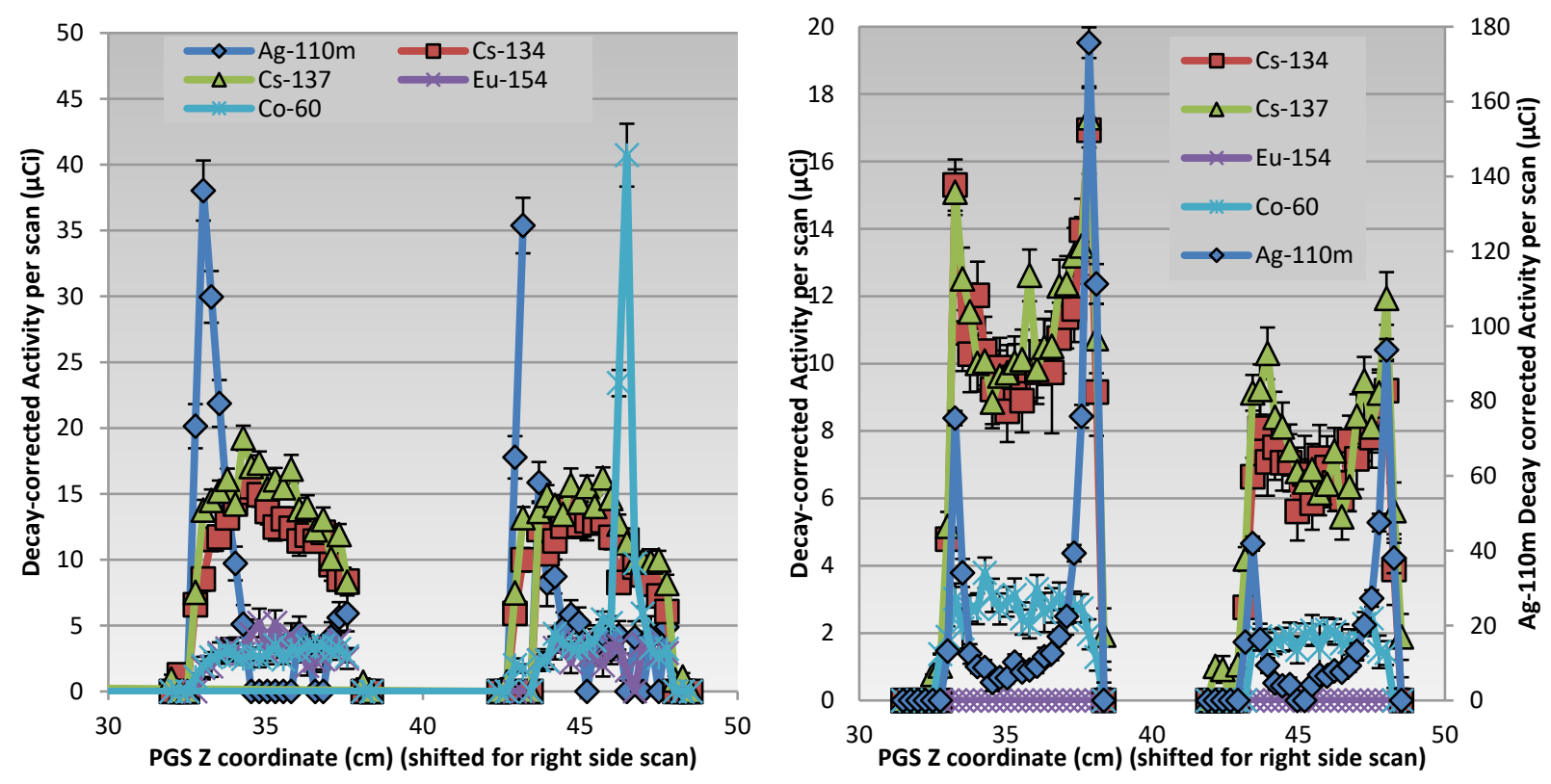

Figure 5. Off-axis scans of the Capsule 3 inner ring (left) and outer ring (right).
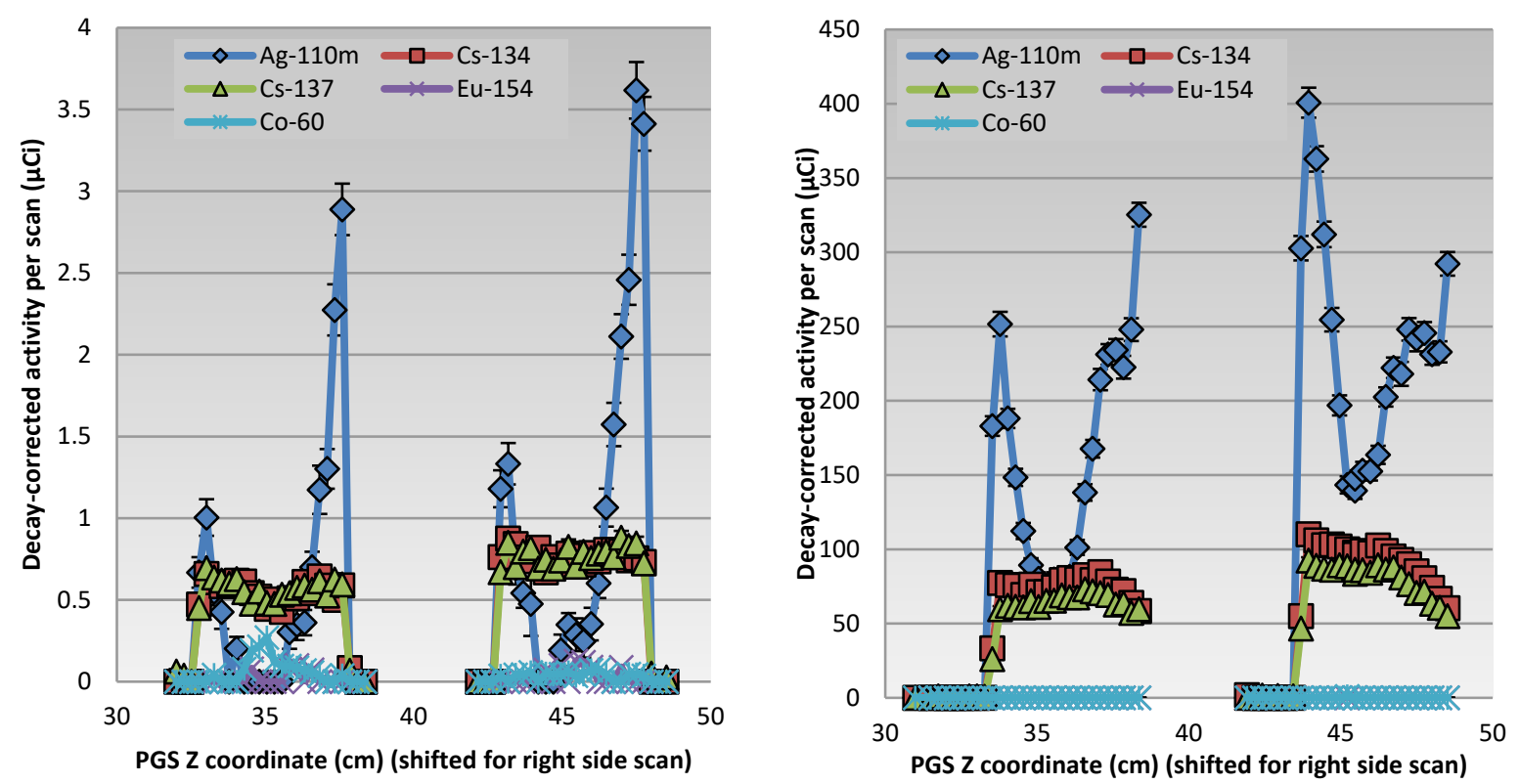

Figure 6. Off-axis scans of the Capsule 8 inner ring (left) and outer ring (right). 
Table 2. Total decay-corrected activity measured in AGR-3/4 inner rings. Uncertainties in parentheses.

\begin{tabular}{c|ccccc|}
\hline & \multicolumn{5}{|c|}{ Inner Ring Activity $(\mu \mathrm{Ci})$} \\
\hline Capsule & $\mathrm{Ag}-110 \mathrm{~m}$ & $\mathrm{Cs}-134$ & $\mathrm{Cs}-137$ & Eu-154 & Co-60 \\
\hline 1 & $0.0(-0.0,+46.7)$ & $75.0(-3.4,+14.8)$ & $242.2(-4.3,+5.6)$ & $4.6(-1.0,+26.0)$ & $7.9(-0.9,+9.8)$ \\
2 & & & & \\
3 & $292.7(-7.6,+27.5)$ & $436.7(-5.7,+6.9)$ & $526.4(-5.4,+5.6)$ & $107.2(-5.0,+10.4)$ & $186.1(-4.0,+4.7)$ \\
4 & $728.6(-31.1,+127.3)$ & $8351.1(-26.5,+27.3)$ & $6459.3(-20.0,+20.1)$ & $0.0(-0.0,+28.1)$ & $4.9(-0.9,+15.1)$ \\
5 & $9.4(-4.1,+112.8)$ & $8309.5(-25.1,+25.4)$ & $5827.4(-19.7,+19.7)$ & $0.0(-0.0,+27.9)$ & $22.3(-1.6,+17.2)$ \\
6 & & & & & \\
7 & $3.6(-1.6,+44.3)$ & $671.7(-8.3,+9.3)$ & $590.3(-6.5,+7.6)$ & $1011.6(-11.5,+14.0)$ & $72.5(-2.4,+7.3)$ \\
8 & $883.7(-17.8,+45.4)$ & $717.6(-8.5,+9.6)$ & $729.3(-7.0,+7.3)$ & $25.3(-3.2,+30.7)$ & $58.8(-2.6,+9.8)$ \\
9 & & & & & \\
10 & $559.6(-13.6,+31.7)$ & $533.5(-7.4,+7.9)$ & $644.0(-6.2,+6.2)$ & $9.4(-1.9,+24.9)$ & $80.1(-2.7,+9.1)$ \\
11 & & & & & $1.8(-0.5,+11.4)$ \\
12 & $0.0(-0.0,+109.9)$ & $29.5(-2.9,+19.9)$ & $185.9(-4.2,+5.2)$ & $0.0(-0.0,+26.9)$ & \\
\hline
\end{tabular}

Table 3. Total decay-corrected activity measured in AGR-3/4 outer rings. Uncertainties in parentheses.

\begin{tabular}{|c|c|c|c|c|c|}
\hline & \multicolumn{5}{|c|}{ Outer Ring $(\mu \mathrm{Ci})$} \\
\hline Capsule & $\mathrm{Ag}-110 \mathrm{~m}$ & Cs-134 & Cs- 137 & Eu-154 & $\mathrm{Co}-60$ \\
\hline 1 & $0.0(-0.0,+46.9)$ & $2.8(-0.7,+14.3)$ & $33.8(-2.1,+6.8)$ & $0.0(-0.0,+25.0)$ & $40.2(-2.1,+13.8)$ \\
\hline \multicolumn{6}{|r|}{ (1) } \\
\hline 3 & $1048.9(-15.9,+24.3)$ & $366.7(-6.3,+7.9)$ & $403.8(-5.1,+5.1)$ & $0.0(-0.0,+26.0)$ & $91.7(-2.8,+4.5)$ \\
\hline 4 & $21.3(-3.0,+73.0)$ & $765.1(-9.1,+9.1)$ & $569.1(-6.6,+6.6)$ & $0.0(-0.0,+27.3)$ & $180.6(-3.5,+3.5)$ \\
\hline 5 & $5.2(-2.5,+68.8)$ & $77.7(-4.1,+11.3)$ & $91.3(-3.1,+11.9)$ & $0.0(-0.0,+25.7)$ & $202.6(-4.5,+11.0)$ \\
\hline \multicolumn{6}{|c|}{ (2) } \\
\hline 7 & $261.0(-9.2,+38.4)$ & $1053.8(-9.5,+10.4)$ & $870.2(-7.6,+8.1)$ & $0.0(-0.0,+26.0)$ & $279.3(-5.1,+6.0)$ \\
\hline 8 & $8107.3(-43.6,+57.2)$ & $3376.5(-16.3,+19.0)$ & $2930.1(-13.5,+14.4)$ & $0.0(-0.0,+30.1)$ & $1.1(-0.4,+11.9)$ \\
\hline \multicolumn{6}{|c|}{ 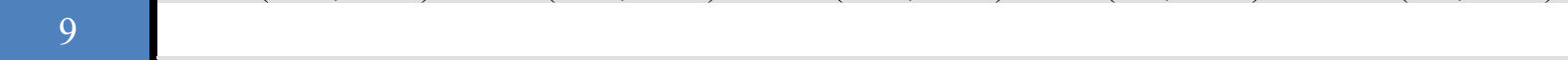 } \\
\hline 10 & $2428.7(-24.9,+27.6)$ & $746.1(-10.0,+10.3)$ & $857.4(-7.3,+7.6)$ & $2.1(-1.0,+25.7)$ & $131.3(-3.5,+18.5)$ \\
\hline \multicolumn{6}{|c|}{ 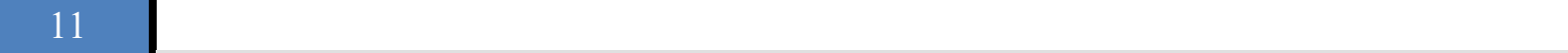 } \\
\hline 12 & $0.0(-0.0,+119.8)$ & $0.0(-0.0,+20.8)$ & $4.3(-0.4,+9.8)$ & $0.0(-0.0,+33.6)$ & $1.3(-0.4,+17.1)$ \\
\hline
\end{tabular}

\subsection{Gamma Emission Computed Tomography}

GECT has been performed on six different inner rings and three outer rings. Of those inner rings, two were scanned at two different axial levels. For the inner rings, scans were performed over 8 to 17 angles with a spatial resolution of $0.127 \mathrm{~cm}$ (the collimator slit size was set to $0.127 \mathrm{~cm}$, and the ring was moved horizontally by the stage by $0.127 \mathrm{~cm}$ each scan), and live times were typically 30 minutes per scan. This results in a scan that lasts from four to nine days, depending on the number of angles scanned. For the outer rings, the scan was performed over eight angles with a spatial resolution of $0.127 \mathrm{~cm}$ and live times of 30 minutes. This resulted in a scan that lasted approximately six days.

A good confirmation of the spatial resolution of the filtered backprojection method employed to produce the GECT maps is shown in Figure 7, which contains the Co-60 tomogram for IR-07. Here the activity is concentrated in a single point corresponding to the location of a melt wire package in IR-07 that had a diameter of $0.137 \mathrm{~cm}$, close to the step size of the scans. The light blue bands that radiate away 
from the point in Figure 7 are artifacts of the filtered backprojection technique called ghosting. These artifacts are a result of the low number of angles (eight in this case) used to create the image. These artifacts are present in all the tomograms presented in Figure 7 through Figure 12. To help distinguish ghosting from a genuine signal, two black circles are also plotted in these images that represent the inner and outer diameter of the scanned rings. Iterative numerical reconstruction methods that identify the ring edge and do not exhibit ghosting are being investigated at INL (Humrickhouse et al. 2018).

The figures following Figure 7 contain a sampling of the observed fission product distributions revealed by GECT. The only fission products observed in any of the rings were Cs-134, Cs-137, Eu-154, and Ag- $110 \mathrm{~m}$. The Cs-134 tomogram from the upper portion of IR-03 in Figure 8 is representative of a somewhat azimuthally uniform Cs-134 distribution. The shading in the tomograms moves from blue to white to red, with blue tones indicating little to no activity and red tones indicating areas of high activity. In these tomograms, the intensity of any pixel is proportional to the amount of activity in the volume represented by that pixel, but the tomograms are not scaled to a quantified activity value. Therefore, the scale is in relative terms specific to that sample only or "Relative Intensity".

The scans that created Figure 8, Figure 9, and Figure 11 had the collimator viewing the upper portion of the ring spanning from the top of the ring to $2.22 \mathrm{~cm}$ below the top of the ring. The Ag- $110 \mathrm{~m}$ tomogram, from the upper portion of IR-07 in Figure 9, shows the generally-observed distribution of Ag$110 \mathrm{~m}$, which does not decrease radially from the ring inner radius to its outer radius. When studying the feasibility of reconstructing AGR-3/4-like profiles using filtered backprojection, a shortcoming that was identified was the difficulty in effectively characterizing flat radial distributions (Harp and Demkowicz 2014). Modeling suggests the Ag-110m may have a radially flat distribution in the inner ring of many capsules. The destructive ring sampling and quantitative analysis should help to better interpret this sort of distribution in the GECT tomograms. A very different distribution is shown for Eu-154 at the midplane of IR-07 in Figure 10. In this ring, the Eu-154 is present at the inner surface of the ring and quickly decreases to nondetectable activities with increasing radius. It should also be noted that the ring is not necessarily perfectly centered in the PGS, which can result in activity appearing partially outside of the expected ring location. The iterative method of reconstruction has also employed corrections for imperfect sample centering in PGS (Humrickhouse et al. 2018).

While both the capsule design and irradiation location in ATR were chosen to minimize azimuthal temperature and flux gradients as much as possible, the GECT results suggest that fission product transport, in some cases, varied around the capsule circumference. Examples of azimuthally nonuniform radial distributions are shown in Figure 11 and Figure 12. Figure 11 shows the Cs-137 distribution in the upper portion of IR-10, and Figure 12 shows the tomogram for Ag-110m at the axial center of OR-08.

In IR-10, there was clearly a greater concentration of Cs-137 activity on one side of the ring, and this can be observed by simply examining the horizontal scans at different angles, indicating that this is a genuine feature and not an artifact of the filtered backprojection method used for this GECT. There is less ghosting in Figure 11 compared to the other tomograms presented here. This set of data was collected over 17 angles (every $11.25^{\circ}$ between 0 and $180^{\circ}$ ), showing the benefit of acquiring data at additional angles, if time allows. As with Figure 11, there are clearly asymmetries in the horizontal scans of OR-08 that were used to create Figure 12. Although Ag-110m was observed in OR-08, it does appear to follow a radially diminishing distribution and azimuthal nonuniformity.

All the data collected in the tomographic scans are tabulated in Appendix D, and the tomograms generated from filtered back projection are shown in 0 . The tomograms shown in this report are reconstructed using a simple application of filtered backprojection. This technique is best used when there are many more angles and much finer spatial resolution; the use of fewer angles results in the ghosting artifacts seen here. An iterative numerical technique that eliminates this is under development at INL (Humrickhouse et al. 2018). 


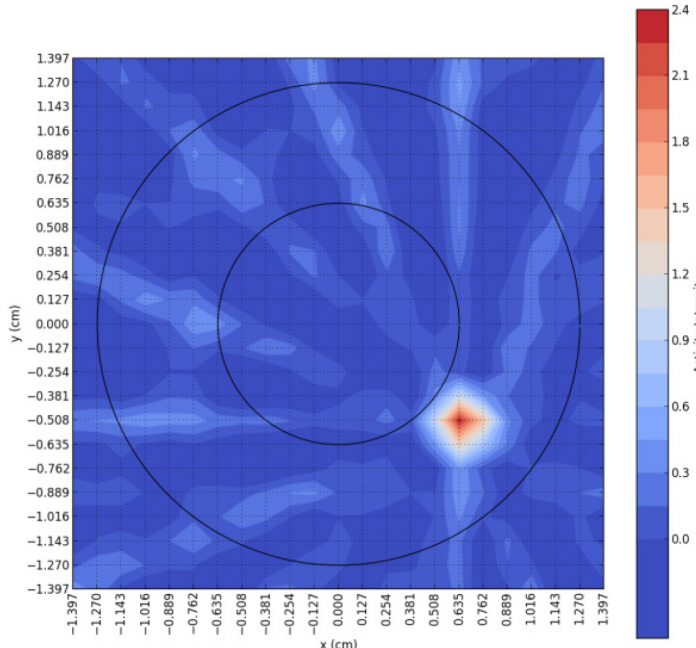

Figure 7. Co-60 tomogram for IR-07 taken at the axial midplane of the ring.

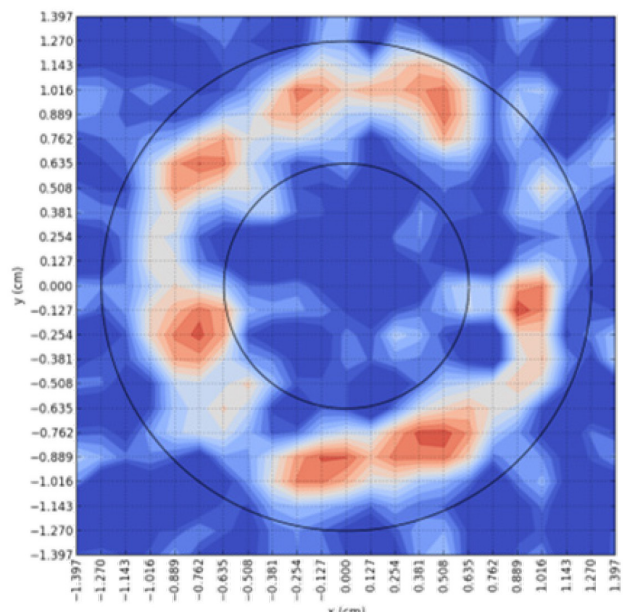

Figure 9. Ag-110m tomogram for the upper level scan from IR-03.

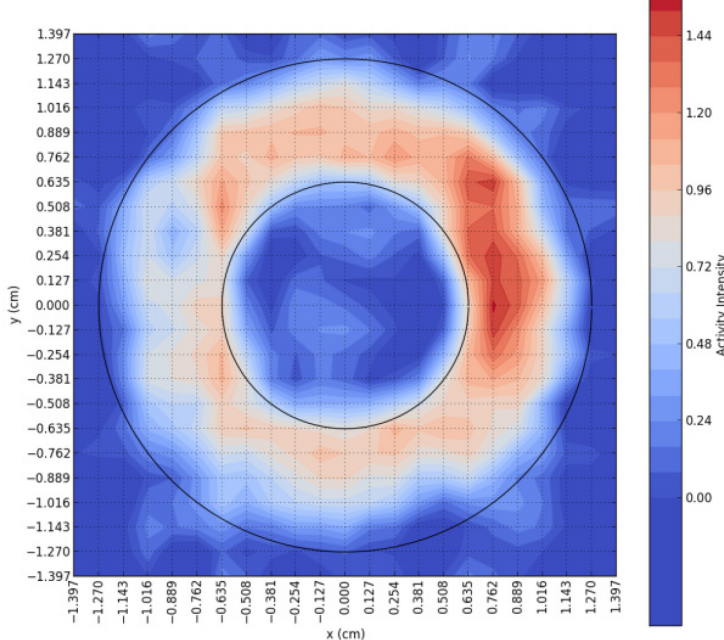

Figure 11. Cs-137 tomogram for the upper level scan from IR-10.

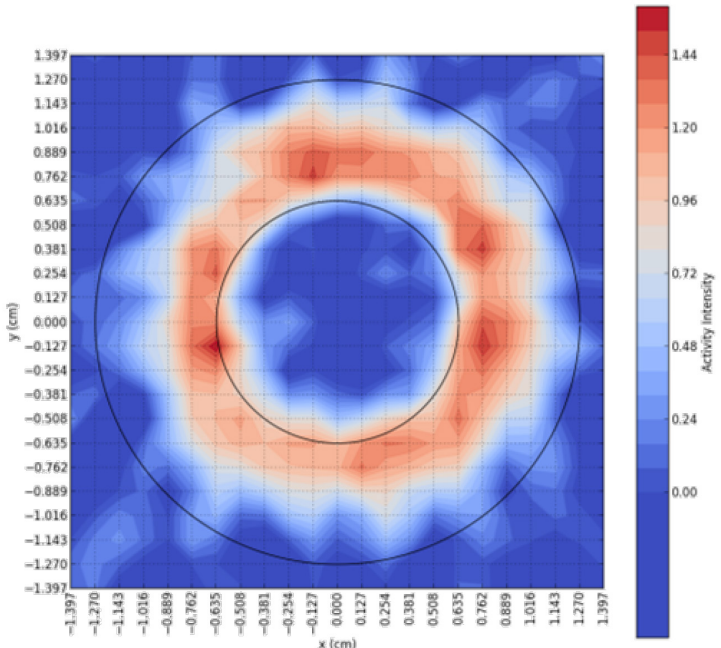

Figure 8. Cs-134 tomogram for the upper level scans from IR-03.

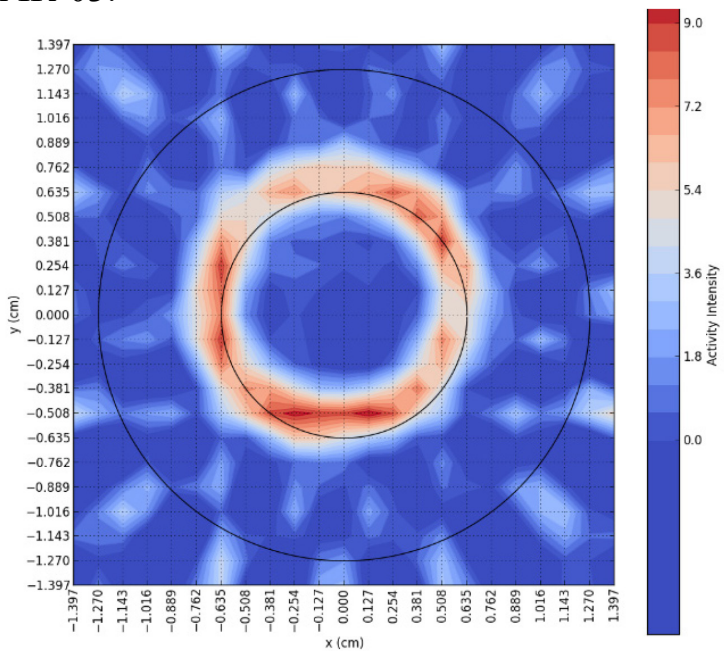

Figure 10. Eu-154 tomogram for the IR-07 taken at the axial midplane of the ring.

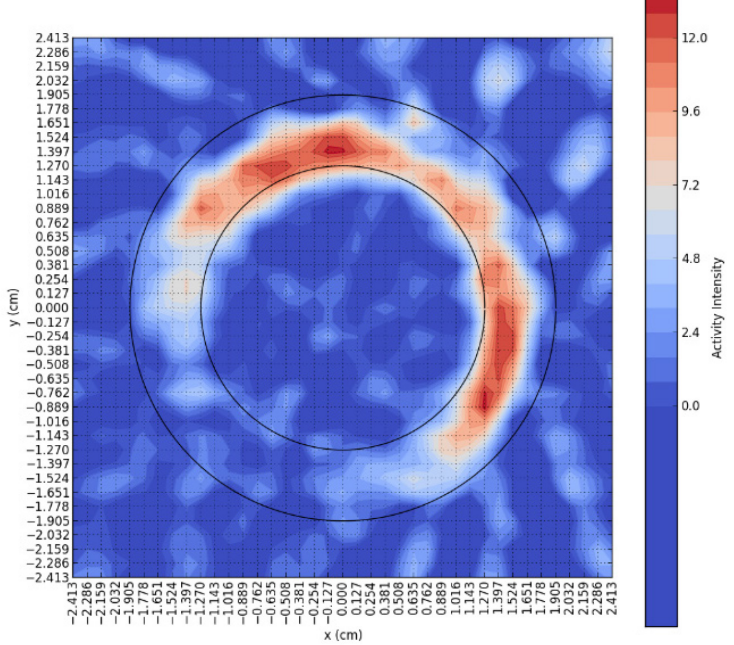

Figure 12. Ag-110m tomogram for the midplane scan of OR-08. 


\subsection{Analytical Laboratory Gamma Scanning}

In addition to scanning with PGS, additional capsule components were sent to the hot cells at the Analytical Laboratory at the INL Materials and Fuels Complex for quantitative gamma spectrometric analysis of fission product inventories outside of the fuel compacts. The Analytical Laboratory also performed analyses to measure Sr-90. These analyses include the graphite sinks from each capsule, which should capture any fission products that have migrated beyond the outer ring of a particular capsule, the capsule through tubes, spacers, felts, and the foils placed at the top and bottom of the stack of compacts in each capsule. These data were used for performing a mass balance on the fission products to be released from the fuel compacts in the capsules (Stempien et al. 2018a). The mass balance is an important parameter for comparing the measured results to those from pre-irradiation simulation predictions of the fission product transport in the AGR-3/4 experiment (Humrickhouse et al. 2016).

\section{CONCLUSIONS}

Gamma spectrometry of the fuel and carbonaceous rings from the AGR-3/4 TRISO fuel irradiation experiment have been completed. Detailed, spatially-resolved scans were performed on fuel compacts and the inner and outer rings using PGS. Gamma spectrometry was used to evaluate the fission product inventory of the fuel compacts from eight of the 12 capsules and also evaluate the burnup of those compacts. (The remaining four fuel-body capsules have been retained intact for future testing.) The agreement between the measured burnup and the burnup predicted from simulations is acceptable. Inner and outer rings from all standard capsules and the Capsule 4 fuel-body have been evaluated by gamma spectrometry as well. Initially, these rings were scanned axially to evaluate the fission product inventory and identify axial locations of interest for additional scanning to evaluate radial distributions. GECT has been applied to evaluate the distribution of fission products at a specified axial level in the rings. This has revealed the distribution of several fission products in these rings, including Ag-110m, Cs-134, Cs-137, and Eu-154. These scans have revealed some axial and azimuthal asymmetries in the migration of fission products through the various rings. The development of a new methodology to transform GECT tomograms into radial fission product distributions is continuing. Destructive sampling of the inner and outer rings from Capsules 3, 5, 7, 8, 10, and 12 is complete. Comparing the results from destructive sampling with results from PGS will allow for the validation of the collected gamma tomography and the creation of radial fission product distributions from non-gamma-emitting fission products (i.e., Sr-90).

\section{REFERENCES}

Barnes, B.K., J.R. Phillips, and M.L. Barnes, 1982, "Reconstruction of Radial Fission Product Distributions in Reactor Fuels from a Small Number of Projections," Journal of Nuclear Materials 106: $147-156$.

Caruso, S. and F. Jatuff, 2014, "Design, Development and Utilisation of a Tomography Station for $\gamma$-Ray Emission and Transmission Analyses of Light Water Reactor Spent Fuel Rods," Progress in Nuclear Energy 72: 49-54.

Collin, B.P., 2015, “AGR-3/4 Irradiation Test Final As-Run Report,” INL/EXT-15-35550, Rev. 0, Idaho National Laboratory.

Collin, B.P., P.A. Demkowicz, D.A. Petti, G.L. Hawkes, J. Palmer, B.T. Pham, D.M. Scates, and J.W. Sterbentz, 2018, "The AGR-3/4 Fission Product Transport Irradiation Experiment," Nuclear Engineering and Design 327: 212-227.

Demkowicz, P.A., J.M. Harp, P.L. Winston, S.A. Ploger, 2013, “Analysis of Fission Products on the AGR-1 Capsule Components," INL/EXT-13-28483, Idaho National Laboratory. 
Demkowicz, P.A., J.D. Hunn, S.A. Ploger, R.N. Morris, C.A. Baldwin, J.M. Harp, P.L. Winston, T.J. Gerczak, I.J. van Rooyen, F.C. Montgomery, C.M. Silva, 2016, "Irradiation Performance of AGR-1 High Temperature Reactor Fuel,” Nuclear Engineering and Design 306: 2-13.

Demkowicz, P.A., 2019, “Technical Program Plan for INL Advanced Reactor Technologies Advanced Gas Reactor Fuel Development and Qualification Program,” PLN-3636, Rev. 8, Idaho National Laboratory.

Dobrin, R., I.L. Tuturici, T. Craciunescu, 1997a, "Gamma Emission Computed Tomography to Identify Defective Nuclear Fuel Rods," in SIEN'97 - International Symposium on Nuclear Energy Radioactive Waste Management, October 24-25, ed. Vasile Plesca (Bucharest, Romania: Energy InfoDocumentation Centre), 284-288.

Dobrin, R., T. Craciunescu, and I.L. Tuturici, 1997b, "The Analysis of Failed Nuclear Fuel Rods by Gamma Computed Tomography,” Journal of Nuclear Materials 246: 37-42.

Harp, J.M. and P.A. Demkowicz, 2014, "Investigation of the Feasibility of Utilizing Gamma Emission Computed Tomography in Evaluating Fission Product Migration in Irradiated TRISO Fuel Experiments," Paper presented at the 7th International Topical Meeting on High Temperature Reactor Technology (HTR2014), Weihai, China, October 2014.

Harp, J.M.,, P.A. Demkowicz, P.L. Winston, and J.W. Sterbentz, 2014, “An Analysis of Nuclear Fuel Burnup in the AGR-1 TRISO Fuel Experiment using Gamma Spectrometry, Mass Spectrometry, and Computational Simulation Techniques," Nuclear Engineering and Design 278: 395-405.

Harp, J.M., P.A. Demkowicz, and J.D. Stempien, 2016, "Initial Gamma Spectrometry Examination of the AGR-3/4 Irradiation," Paper HTR2016-18593, 8th International Topical Meeting on High Temperature Reactor Technology (HTR2016), Las Vegas, NV, USA, November 2016.

Harp, J.M. and H.J.M Chichester, 2016, "Highlights from the Postirradiation Examination of AFC-3A and AFC-3B," in Transactions of the American Nuclear Society, Vol. 114.

Harp, J.M., P.A. Demkowicz, and J.D. Stempien, 2020, "Fission Product Inventory and Burnup Evaluation by Gamma Spectrometry of the AGR-2 Irradiation," INL/EXT-16-39777, Rev. 1, Idaho National Laboratory.

Humrickhouse, P.W., B.P. Collin, G.L. Hawkes, J.M. Harp, P.A. Demkowicz, and D.A. Petti, 2016, "Modeling and Analysis of Fission Product Transport in the AGR-3/4 Experiment," Paper presented at the $8^{\text {th }}$ International Topical Meeting on High Temperature Reactor Technology (HTR 2016), Las Vegas, Nevada, November 2016. Paper HTR2016-18693.

Humrickhouse, P.W., J.D. Stempien, J.M. Harp, P.A. Demkowicz, and D.A. Petti, 2018, "Preliminary Estimation of Fission Product Diffusion Coefficients from AGR-3/4 Data," Paper presented at the $9^{\text {th }}$ International Topical Meeting on High Temperature Reactor Technology (HTR-2018), Warsaw, Poland, October 2018.

Hunn, J.D., M.P. Trammell, and F.C. Montgomery, 2011, "Data Compilation for AGR-3/4 Designed-toFail (DTF) Fuel Compact Lot (LEU03-10T-OP2/LEU03-07DTF-OP1)-Z,” ORNL/TM-2011/124, Oak Ridge National Laboratory.

Hunn, J.D., C.A. Baldwin, T.J. Gerczak, F.C. Montgomery, R.N. Morris, C.M. Silva, P.A. Demkowicz, J.M. Harp, and S.A. Ploger, 2016, "Detection and Analysis of Particles with Failed SiC in AGR-1 Fuel Compacts," Nuclear Engineering and Design 306: 36-46.

Morris, R.N., C.A. Baldwin, P.A. Demkowicz, J.D. Hunn, and E.L. Reber, 2016, "Performance of AGR-1 High Temperature Reactor Fuel During Post Irradiation Heating Tests," Nuclear Engineering and Design 306: 24-35. 
Petti, D.A., B.P. Collin, and D. Marshall, 2017, “A Summary of the Results from the DOE Advanced Gas Reactor (AGR) Fuel Development and Qualification Program,” INL/EXT-16-40784, Idaho National Laboratory.

Stempien, J.D., F.J. Rice, P.L. Winston, and J.M. Harp, 2016, “AGR-3/4 Irradiation Test Train Disassembly and Component Metrology First Look Report,” INL/EXT-16-38005, Rev. 1, Idaho National Laboratory.

Stempien, J.D., P.A. Demkowicz, J.M. Harp, and P.L. Winston, 2018a, “AGR-3/4 Experiment Preliminary Mass Balance,” INL/EXT-18-46049, Idaho National Laboratory.

Stempien, J.D., P.A. Demkowicz, and P.L. Winston, 2018b, "Preliminary Results of Post-Irradiation Examination of the AGR-3/4 Irradiation to Assess Fission Product Mobility in Graphite and Graphitic Matrix Material," Paper presented at the $9^{\text {th }}$ International Topical Meeting on High Temperature Reactor Technology (HTR-2018), Warsaw, Poland, October 2018.

Stempien, J.D. and P.A. Demkowicz, 2020, "AGR-2 Irradiation Experiment Fission Product Mass Balance,” INL/EXT-19-53559, Rev. 1, Idaho National Laboratory.

Sterbentz, J.W., 2015, "JMOCUP As-Run Daily Physics Depletion Calculation for the AGR-3/4 TRISO Particle Experiment in ATR Northeast Flux Trap," ECAR-2753, Idaho National Laboratory, July 2015.

Wernick, M.N, and J.N. Aarsvold, 2004, Emission Tomography: The Fundamentals of PET and SPECT. Amsterdam: Elsevier Academic Press. 
Appendix A Burnup Conversion and Total Compact Activity for Selected Isotopes

Table A.1. Activity and activity ratio to burnup conversion coefficients in \% FIMA.

\begin{tabular}{|c|c|c|}
\hline & Cs-134 : Cs-137 Ratio & Cs-137 Total Compact Activity \\
\hline Slope $(m)$ & $9.98 \mathrm{E}+00$ & $7.33 \mathrm{E}-05\left(\mu \mathrm{Ci}^{-1}\right)$ \\
\hline Intercept $(\mathrm{b})$ & $1.70 \mathrm{E}+00$ & $6.35 \mathrm{E}-02$ \\
\hline $\begin{array}{l}\text { Burnup (\% FIMA) }=\mathrm{mx}+\mathrm{b} \\
\mathrm{x}=\mathrm{Cs}-134: \text { Cs-137 or Cs-137 activity }\end{array}$ \\
\hline
\end{tabular}


Table A.2. Measured decay-corrected activities and uncertainties ( $\Delta$ ) for Ag-110m, Cs-134, Cs-137, Eu-154, Zr-95, Ru-106, and Ce-144.

\begin{tabular}{|c|c|c|c|c|c|c|c|c|c|c|c|c|c|c|c|}
\hline \multirow{2}{*}{ 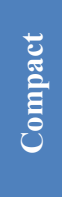 } & \multicolumn{3}{|c|}{ Ag-110m } & \multicolumn{2}{|c|}{ Cs-134 } & \multicolumn{2}{|c|}{ Cs-137 } & \multicolumn{2}{|c|}{ Eu-154 } & \multicolumn{2}{|c|}{$\mathrm{Zr}-95$} & \multicolumn{2}{|c|}{ Ru-106 } & \multicolumn{2}{|c|}{ Ce-144 } \\
\hline & $\begin{array}{c}\text { Activity } \\
(\mu \mathrm{Ci})\end{array}$ & $+\Delta(\mu \mathrm{Ci})$ & $-\Delta(\mu \mathrm{Ci})$ & $\begin{array}{c}\text { Activity } \\
(\mu \mathrm{Ci})\end{array}$ & $\pm \Delta(\mu \mathrm{Ci})$ & $\begin{array}{c}\text { Activity } \\
(\mu \mathrm{Ci})\end{array}$ & $\pm \Delta(\mu \mathrm{Ci})$ & $\begin{array}{c}\text { Activity } \\
(\mu \mathrm{Ci})\end{array}$ & $\pm \Delta(\mu \mathrm{Ci})$ & $\mid \begin{array}{c}\text { Activity } \\
(\mu \mathrm{Ci})\end{array}$ & $\pm \Delta(\mu \mathrm{Ci})$ & $\mid \begin{array}{c}\text { Activity } \\
(\mu \mathrm{Ci})\end{array}$ & $\pm \Delta(\mu \mathrm{Ci})$ & $\begin{array}{c}\text { Activity } \\
(\mu \mathrm{Ci})\end{array}$ & $\pm \Delta(\mu \mathrm{Ci})$ \\
\hline $1-4$ & $2.60 \mathrm{E}+02$ & $2.17 \mathrm{E}+01$ & $2.17 \mathrm{E}+01$ & $4.72 \mathrm{E}+04$ & $7.08 \mathrm{E}+01$ & $9.79 \mathrm{E}+04$ & $1.21 \mathrm{E}+02$ & $1.43 \mathrm{E}+03$ & $1.84 \mathrm{E}+01$ & $2.98 \mathrm{E}+06$ & $1.48 \mathrm{E}+04$ & $2.16 \mathrm{E}+05$ & $4.54 \mathrm{E}+02$ & $1.62 \mathrm{E}+06$ & $6.08 \mathrm{E}+03$ \\
\hline $1-3$ & $2.07 \mathrm{E}+02$ & $2.21 \mathrm{E}+01$ & $2.21 \mathrm{E}+01$ & $4.04 \mathrm{E}+04$ & $5.95 \mathrm{E}+01$ & $9.15 E+04$ & $1.09 \mathrm{E}+02$ & $1.25 \mathrm{E}+03$ & $1.68 \mathrm{E}+01$ & $2.78 \mathrm{E}+06$ & $1.65 \mathrm{E}+04$ & $1.92 \mathrm{E}+05$ & $4.44 \mathrm{E}+02$ & $1.52 \mathrm{E}+06$ & $5.83 \mathrm{E}+03$ \\
\hline $1-2$ & $1.09 \mathrm{E}+02$ & $4.45 \mathrm{E}+01$ & $1.74 \mathrm{E}+01$ & $3.40 \mathrm{E}+04$ & $5.51 \mathrm{E}+01$ & $8.53 \mathrm{E}+04$ & $9.59 \mathrm{E}+01$ & $1.06 \mathrm{E}+03$ & $1.68 \mathrm{E}+01$ & $2.63 \mathrm{E}+06$ & $1.46 \mathrm{E}+04$ & $1.71 \mathrm{E}+05$ & $4.30 \mathrm{E}+02$ & $1.43 \mathrm{E}+06$ & $5.95 \mathrm{E}+03$ \\
\hline $1-1$ & $1.09 \mathrm{E}+02$ & $3.98 \mathrm{E}+01$ & $1.84 \mathrm{E}+01$ & $2.70 \mathrm{E}+04$ & $4.78 \mathrm{E}+01$ & $7.73 E+04$ & $1.10 \mathrm{E}+02$ & $6.74 \mathrm{E}+02$ & $1.33 \mathrm{E}+01$ & $2.42 \mathrm{E}+06$ & $1.64 \mathrm{E}+04$ & $1.47 \mathrm{E}+05$ & $3.84 \mathrm{E}+02$ & $1.29 \mathrm{E}+06$ & $5.85 \mathrm{E}+03$ \\
\hline 3-4 & $9.88 \mathrm{E}+01$ & $1.07 \mathrm{E}+02$ & $2.36 \mathrm{E}+01$ & $1.80 \mathrm{E}+05$ & $1.94 \mathrm{E}+02$ & $1.75 \mathrm{E}+05$ & $2.38 \mathrm{E}+02$ & $5.34 \mathrm{E}+03$ & $3.44 \mathrm{E}+01$ & $4.75 \mathrm{E}+06$ & $2.30 \mathrm{E}+04$ & $5.77 \mathrm{E}+05$ & $9.13 \mathrm{E}+02$ & $2.70 \mathrm{E}+06$ & $8.46 \mathrm{E}+03$ \\
\hline 3-3 & $1.76 \mathrm{E}+02$ & $7.59 \mathrm{E}+01$ & $2.86 \mathrm{E}+01$ & $1.84 \mathrm{E}+05$ & $2.08 \mathrm{E}+02$ & $1.82 \mathrm{E}+05$ & $2.62 \mathrm{E}+02$ & $5.40 \mathrm{E}+03$ & $3.48 \mathrm{E}+01$ & $4.99 \mathrm{E}+06$ & $2.24 \mathrm{E}+04$ & $5.86 \mathrm{E}+05$ & $9.85 \mathrm{E}+02$ & $2.80 \mathrm{E}+06$ & $9.17 \mathrm{E}+03$ \\
\hline $3-2$ & $1.29 \mathrm{E}+02$ & $8.78 \mathrm{E}+01$ & $2.81 \mathrm{E}+01$ & $1.76 \mathrm{E}+05$ & $2.03 \mathrm{E}+02$ & $1.78 \mathrm{E}+05$ & $2.45 \mathrm{E}+02$ & $5.23 \mathrm{E}+03$ & $3.38 \mathrm{E}+01$ & $4.84 \mathrm{E}+06$ & $2.42 \mathrm{E}+04$ & $5.65 \mathrm{E}+05$ & $1.00 \mathrm{E}+03$ & $2.74 \mathrm{E}+06$ & $7.56 \mathrm{E}+03$ \\
\hline $4-4$ & $1.99 \mathrm{E}+03$ & $7.04 \mathrm{E}+01$ & $7.04 \mathrm{E}+01$ & $2.40 \mathrm{E}+05$ & $5.43 \mathrm{E}+02$ & $2.00 \mathrm{E}+05$ & $3.10 \mathrm{E}+02$ & $6.42 \mathrm{E}+03$ & $3.43 \mathrm{E}+01$ & $1.20 \mathrm{E}+07$ & $2.54 \mathrm{E}+05$ & $6.97 \mathrm{E}+05$ & $1.57 \mathrm{E}+03$ & $3.03 \mathrm{E}+06$ & $1.57 \mathrm{E}+04$ \\
\hline $4-3$ & $1.82 \mathrm{E}+03$ & $7.68 \mathrm{E}+01$ & $7.68 \mathrm{E}+01$ & $2.34 \mathrm{E}+05$ & $5.41 \mathrm{E}+02$ & $1.96 \mathrm{E}+05$ & $3.18 \mathrm{E}+02$ & $6.15 \mathrm{E}+03$ & $3.52 \mathrm{E}+01$ & $1.14 \mathrm{E}+07$ & $2.28 \mathrm{E}+05$ & $6.71 \mathrm{E}+05$ & $1.40 \mathrm{E}+03$ & $2.95 \mathrm{E}+06$ & $1.20 \mathrm{E}+04$ \\
\hline $4-2$ & $1.87 \mathrm{E}+03$ & $7.12 \mathrm{E}+01$ & $7.12 \mathrm{E}+01$ & $2.31 \mathrm{E}+05$ & $4.28 \mathrm{E}+02$ & $1.97 \mathrm{E}+05$ & $2.96 \mathrm{E}+02$ & $6.17 \mathrm{E}+03$ & $3.52 \mathrm{E}+01$ & $1.19 \mathrm{E}+07$ & $2.28 \mathrm{E}+05$ & $6.67 \mathrm{E}+05$ & $1.50 \mathrm{E}+03$ & $2.98 \mathrm{E}+06$ & $1.14 \mathrm{E}+04$ \\
\hline $4-1$ & $1.68 \mathrm{E}+03$ & $6.71 \mathrm{E}+01$ & $6.71 \mathrm{E}+01$ & $1.94 \mathrm{E}+05$ & $3.72 \mathrm{E}+02$ & $1.69 \mathrm{E}+05$ & $2.79 \mathrm{E}+02$ & $5.23 \mathrm{E}+03$ & $3.17 \mathrm{E}+01$ & $9.50 \mathrm{E}+06$ & $2.26 \mathrm{E}+05$ & $5.66 \mathrm{E}+05$ & $1.24 \mathrm{E}+03$ & $2.52 \mathrm{E}+06$ & $1.24 \mathrm{E}+04$ \\
\hline $5-4$ & $2.24 \mathrm{E}+03$ & $7.69 \mathrm{E}+01$ & $7.69 \mathrm{E}+01$ & $2.49 \mathrm{E}+05$ & $5.07 \mathrm{E}+02$ & $1.95 \mathrm{E}+05$ & $3.59 \mathrm{E}+02$ & $6.55 \mathrm{E}+03$ & $3.87 \mathrm{E}+01$ & $1.26 \mathrm{E}+07$ & $2.59 \mathrm{E}+05$ & $6.98 \mathrm{E}+05$ & $1.58 \mathrm{E}+03$ & $2.97 \mathrm{E}+06$ & $1.29 \mathrm{E}+04$ \\
\hline $5-3$ & $2.42 \mathrm{E}+03$ & $8.48 \mathrm{E}+01$ & $8.48 \mathrm{E}+01$ & $2.61 \mathrm{E}+05$ & $5.15 \mathrm{E}+02$ & $2.06 \mathrm{E}+05$ & $3.17 \mathrm{E}+02$ & $6.91 \mathrm{E}+03$ & $3.96 \mathrm{E}+01$ & $1.23 \mathrm{E}+07$ & $2.37 \mathrm{E}+05$ & $7.36 \mathrm{E}+05$ & $1.71 \mathrm{E}+03$ & $3.07 \mathrm{E}+06$ & $1.59 \mathrm{E}+04$ \\
\hline $5-2$ & $2.50 \mathrm{E}+03$ & $8.51 \mathrm{E}+01$ & $8.51 \mathrm{E}+01$ & $2.63 \mathrm{E}+05$ & $4.26 \mathrm{E}+02$ & $2.08 \mathrm{E}+05$ & $2.83 \mathrm{E}+02$ & $6.94 \mathrm{E}+03$ & $3.53 \mathrm{E}+01$ & $1.26 \mathrm{E}+07$ & $2.42 \mathrm{E}+05$ & $7.39 \mathrm{E}+05$ & $1.51 \mathrm{E}+03$ & $3.11 \mathrm{E}+06$ & $1.32 \mathrm{E}+04$ \\
\hline $5-1$ & $2.08 \mathrm{E}+03$ & $7.41 \mathrm{E}+01$ & $7.41 \mathrm{E}+01$ & $2.27 \mathrm{E}+05$ & $4.27 \mathrm{E}+02$ & $1.78 \mathrm{E}+05$ & $2.50 \mathrm{E}+02$ & $5.98 \mathrm{E}+03$ & $3.70 \mathrm{E}+01$ & $1.08 \mathrm{E}+07$ & $2.28 \mathrm{E}+05$ & $6.36 \mathrm{E}+05$ & $1.66 \mathrm{E}+03$ & $2.68 \mathrm{E}+06$ & $1.18 \mathrm{E}+04$ \\
\hline $7-4$ & $4.69 \mathrm{E}+02$ & $3.58 \mathrm{E}+01$ & $3.58 \mathrm{E}+01$ & $2.68 \mathrm{E}+05$ & $2.87 \mathrm{E}+02$ & $2.16 \mathrm{E}+05$ & $2.86 \mathrm{E}+02$ & $7.29 \mathrm{E}+03$ & $3.78 \mathrm{E}+01$ & $5.53 \mathrm{E}+06$ & $2.45 \mathrm{E}+04$ & $7.99 \mathrm{E}+05$ & $1.34 \mathrm{E}+03$ & $3.23 \mathrm{E}+06$ & $9.54 \mathrm{E}+03$ \\
\hline $7-3$ & $3.87 \mathrm{E}+02$ & $5.21 \mathrm{E}+01$ & $4.44 \mathrm{E}+01$ & $2.69 \mathrm{E}+05$ & $2.70 \mathrm{E}+02$ & $2.13 \mathrm{E}+05$ & $3.02 \mathrm{E}+02$ & $7.26 \mathrm{E}+03$ & $3.82 \mathrm{E}+01$ & $5.45 \mathrm{E}+06$ & $2.10 \mathrm{E}+04$ & $7.86 \mathrm{E}+05$ & $1.18 \mathrm{E}+03$ & $3.15 \mathrm{E}+06$ & $9.83 \mathrm{E}+03$ \\
\hline
\end{tabular}




\begin{tabular}{|c|c|c|c|c|c|c|c|c|c|c|c|c|c|c|c|}
\hline \multirow{2}{*}{ 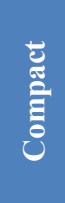 } & \multicolumn{3}{|c|}{ Ag-110m } & \multicolumn{2}{|c|}{ Cs-134 } & \multicolumn{2}{|c|}{ Cs-137 } & \multicolumn{2}{|c|}{ Eu-154 } & \multicolumn{2}{|c|}{ Zr-95 } & \multicolumn{2}{|c|}{ Ru-106 } & \multicolumn{2}{|c|}{ Ce-144 } \\
\hline & $\begin{array}{c}\text { Activity } \\
(\mu \mathrm{Ci})\end{array}$ & $+\Delta(\mu \mathrm{Ci})$ & $-\Delta(\mu \mathrm{Ci})$ & $\begin{array}{c}\text { Activity } \\
(\mu \mathrm{Ci})\end{array}$ & $\pm \Delta(\mu \mathrm{Ci})$ & $\begin{array}{c}\text { Activity } \\
(\mu \mathrm{Ci})\end{array}$ & $\pm \Delta(\mu \mathrm{Ci})$ & $\mid \begin{array}{c}\text { Activity } \\
(\mu \mathrm{C} i)\end{array}$ & $\pm \Delta(\mu \mathrm{Ci})$ & $\mid \begin{array}{c}\text { Activity } \\
(\mu \mathrm{Ci})\end{array}$ & $\pm \Delta(\mu \mathrm{Ci})$ & $\mid \begin{array}{c}\text { Activity } \\
(\mu \mathrm{Ci})\end{array}$ & $\pm \Delta(\mu \mathrm{Ci})$ & $\begin{array}{c}\text { Activity } \\
(\mu \mathrm{C} i)\end{array}$ & $\pm \Delta(\mu \mathrm{Ci})$ \\
\hline $7-1$ & $4.01 \mathrm{E}+02$ & $4.58 \mathrm{E}+01$ & $4.35 \mathrm{E}+01$ & $2.75 \mathrm{E}+05$ & $2.97 \mathrm{E}+02$ & $2.20 \mathrm{E}+05$ & $2.91 \mathrm{E}+02$ & $6.06 \mathrm{E}+03$ & $3.45 \mathrm{E}+01$ & $5.56 \mathrm{E}+06$ & $2.34 \mathrm{E}+04$ & $8.17 \mathrm{E}+05$ & $1.18 \mathrm{E}+03$ & $3.24 \mathrm{E}+06$ & $8.33 \mathrm{E}+03$ \\
\hline $8-4$ & $4.49 \mathrm{E}+02$ & $4.61 \mathrm{E}+01$ & $4.61 \mathrm{E}+01$ & $2.45 \mathrm{E}+05$ & $4.95 \mathrm{E}+02$ & $2.07 \mathrm{E}+05$ & $5.89 \mathrm{E}+02$ & $6.87 \mathrm{E}+03$ & $3.68 \mathrm{E}+01$ & $5.89 \mathrm{E}+06$ & $4.81 \mathrm{E}+04$ & $7.25 \mathrm{E}+05$ & $1.62 \mathrm{E}+03$ & $3.07 \mathrm{E}+06$ & $1.04 \mathrm{E}+04$ \\
\hline $8-3$ & $4.68 \mathrm{E}+02$ & $4.83 \mathrm{E}+01$ & $4.83 \mathrm{E}+01$ & $2.54 \mathrm{E}+05$ & $4.78 \mathrm{E}+02$ & $2.10 \mathrm{E}+05$ & $5.14 \mathrm{E}+02$ & $7.11 \mathrm{E}+03$ & $3.92 \mathrm{E}+01$ & $5.91 \mathrm{E}+06$ & $4.42 \mathrm{E}+04$ & $7.37 \mathrm{E}+05$ & $1.77 \mathrm{E}+03$ & $3.11 \mathrm{E}+06$ & $9.33 \mathrm{E}+03$ \\
\hline $8-2$ & $4.16 \mathrm{E}+02$ & $1.07 \mathrm{E}+02$ & $4.61 \mathrm{E}+01$ & $2.54 \mathrm{E}+05$ & $5.04 \mathrm{E}+02$ & $2.09 \mathrm{E}+05$ & $5.37 \mathrm{E}+02$ & $7.05 \mathrm{E}+03$ & $3.99 \mathrm{E}+01$ & $5.86 \mathrm{E}+06$ & $4.02 \mathrm{E}+04$ & $7.39 \mathrm{E}+05$ & $1.73 \mathrm{E}+03$ & $3.09 \mathrm{E}+06$ & $1.35 \mathrm{E}+04$ \\
\hline 8-1 & $5.18 \mathrm{E}+02$ & $6.95 \mathrm{E}+01$ & $5.83 \mathrm{E}+01$ & $2.54 \mathrm{E}+05$ & $5.02 \mathrm{E}+02$ & $2.11 \mathrm{E}+05$ & $5.60 \mathrm{E}+02$ & $7.11 \mathrm{E}+03$ & $4.21 \mathrm{E}+01$ & $5.93 \mathrm{E}+06$ & $4.39 \mathrm{E}+04$ & $7.54 \mathrm{E}+05$ & $1.72 \mathrm{E}+03$ & $3.16 \mathrm{E}+06$ & $1.40 \mathrm{E}+04$ \\
\hline $10-4$ & $6.93 \mathrm{E}+01$ & $1.49 \mathrm{E}+02$ & $2.30 \mathrm{E}+01$ & $1.35 \mathrm{E}+05$ & $3.01 \mathrm{E}+02$ & $1.56 \mathrm{E}+05$ & $4.31 \mathrm{E}+02$ & $4.02 \mathrm{E}+03$ & $3.08 \mathrm{E}+01$ & $4.67 \mathrm{E}+06$ & $3.38 \mathrm{E}+04$ & $4.67 \mathrm{E}+05$ & $1.25 \mathrm{E}+03$ & $2.44 \mathrm{E}+06$ & $9.38 \mathrm{E}+03$ \\
\hline $10-3$ & $1.28 \mathrm{E}+02$ & $1.23 \mathrm{E}+02$ & $2.12 \mathrm{E}+01$ & $1.47 \mathrm{E}+05$ & $3.18 \mathrm{E}+02$ & $1.62 \mathrm{E}+05$ & $4.35 \mathrm{E}+02$ & $4.34 \mathrm{E}+03$ & $2.96 \mathrm{E}+01$ & $4.78 \mathrm{E}+06$ & $4.12 \mathrm{E}+04$ & $4.92 \mathrm{E}+05$ & $1.34 \mathrm{E}+03$ & $2.50 \mathrm{E}+06$ & $9.67 \mathrm{E}+03$ \\
\hline $10-2$ & $3.13 \mathrm{E}+02$ & $5.27 \mathrm{E}+01$ & $3.80 \mathrm{E}+01$ & $1.56 \mathrm{E}+05$ & $3.20 \mathrm{E}+02$ & $1.67 \mathrm{E}+05$ & $4.35 \mathrm{E}+02$ & $4.63 \mathrm{E}+03$ & $3.04 \mathrm{E}+01$ & $4.93 \mathrm{E}+06$ & $3.93 \mathrm{E}+04$ & $5.16 \mathrm{E}+05$ & $1.29 \mathrm{E}+03$ & $2.57 \mathrm{E}+06$ & $9.22 \mathrm{E}+03$ \\
\hline 10-1 & $2.59 \mathrm{E}+02$ & $8.70 \mathrm{E}+01$ & $3.79 \mathrm{E}+01$ & $1.62 \mathrm{E}+05$ & $3.83 \mathrm{E}+02$ & $1.72 \mathrm{E}+05$ & $4.86 \mathrm{E}+02$ & $4.78 \mathrm{E}+03$ & $3.30 \mathrm{E}+01$ & $5.04 \mathrm{E}+06$ & $4.17 \mathrm{E}+04$ & $5.38 \mathrm{E}+05$ & $1.44 \mathrm{E}+03$ & $2.64 \mathrm{E}+06$ & $1.06 \mathrm{E}+04$ \\
\hline $12-4$ & $2.26 \mathrm{E}+01$ & $7.52 \mathrm{E}+01$ & $9.72 \mathrm{E}+00$ & $1.63 \mathrm{E}+04$ & $4.73 \mathrm{E}+01$ & $5.91 \mathrm{E}+04$ & $1.86 \mathrm{E}+02$ & $5.01 \mathrm{E}+02$ & $1.29 \mathrm{E}+01$ & $1.89 \mathrm{E}+06$ & $2.86 \mathrm{E}+04$ & $1.03 \mathrm{E}+05$ & $3.92 \mathrm{E}+02$ & $9.94 \mathrm{E}+05$ & $5.28 \mathrm{E}+03$ \\
\hline $12-3$ & $2.83 \mathrm{E}+01$ & $8.54 \mathrm{E}+01$ & $1.01 \mathrm{E}+01$ & $2.11 \mathrm{E}+04$ & $5.55 \mathrm{E}+01$ & $6.59 \mathrm{E}+04$ & $1.84 \mathrm{E}+02$ & $6.50 \mathrm{E}+02$ & $1.32 \mathrm{E}+01$ & $2.10 \mathrm{E}+06$ & $2.90 \mathrm{E}+04$ & $1.21 \mathrm{E}+05$ & $4.42 \mathrm{E}+02$ & $1.10 \mathrm{E}+06$ & $5.19 \mathrm{E}+03$ \\
\hline $12-2$ & $7.46 \mathrm{E}+01$ & $6.75 \mathrm{E}+01$ & $1.77 \mathrm{E}+01$ & $2.64 \mathrm{E}+04$ & $6.74 \mathrm{E}+01$ & $7.41 \mathrm{E}+04$ & $2.08 \mathrm{E}+02$ & $8.22 \mathrm{E}+02$ & $1.37 \mathrm{E}+01$ & $2.35 \mathrm{E}+06$ & $2.34 \mathrm{E}+04$ & $1.42 \mathrm{E}+05$ & $4.83 \mathrm{E}+02$ & $1.24 \mathrm{E}+06$ & $6.82 \mathrm{E}+03$ \\
\hline $12-1$ & $1.41 \mathrm{E}+02$ & $5.41 \mathrm{E}+01$ & $2.40 \mathrm{E}+01$ & $3.11 \mathrm{E}+04$ & $7.72 \mathrm{E}+01$ & $8.02 \mathrm{E}+04$ & $2.25 \mathrm{E}+02$ & $9.66 \mathrm{E}+02$ & $1.65 \mathrm{E}+01$ & $2.53 \mathrm{E}+06$ & $2.43 \mathrm{E}+04$ & $1.62 \mathrm{E}+05$ & $5.61 \mathrm{E}+02$ & $1.32 \mathrm{E}+06$ & $6.67 \mathrm{E}+03$ \\
\hline
\end{tabular}




\section{Appendix B Measured Versus Calculated Burnup for Each Compact}

\begin{tabular}{|c|c|c|c|}
\hline Compact & $\begin{array}{c}\text { Compact Average Experimental } \\
\text { Burnup from Cs-134:Cs-137 } \\
\text { Ratio Method (\% FIMA) }\end{array}$ & $\begin{array}{l}\text { Compact Experimental } \\
\text { Burnup from Total Cs- } \\
137 \text { Activity (\% FIMA) }\end{array}$ & $\begin{array}{c}\text { Calculated Burnup from } \\
\text { Simulation (\%FIMA) } \\
\text { (Sterbentz 2015) }\end{array}$ \\
\hline $1-4$ & 6.5 & 7.2 & 6.85 \\
\hline $1-3$ & 6.1 & 6.8 & 6.37 \\
\hline $1-2$ & 5.7 & 6.3 & 5.91 \\
\hline $1-1$ & 5.2 & 5.7 & 5.43 \\
\hline $3-4$ & 12.0 & 12.9 & 12.93 \\
\hline 3-3 & 11.8 & 13.4 & 12.73 \\
\hline $3-2$ & 11.5 & 13.1 & 12.49 \\
\hline $3-1$ & 11.2 & 12.7 & 12.16 \\
\hline 4-4 & 13.7 & 14.7 & 14.41 \\
\hline 4-3 & 13.7 & 14.4 & 14.29 \\
\hline 4-2 & 13.4 & 14.5 & 14.16 \\
\hline 4-1 & 13.1 & 12.4 & 13.98 \\
\hline $5-4$ & 14.5 & 14.4 & 14.98 \\
\hline $5-3$ & 14.3 & 15.2 & 14.92 \\
\hline $5-2$ & 14.4 & 15.3 & 14.86 \\
\hline $5-1$ & 14.2 & 13.8 & 14.74 \\
\hline $7-4$ & 14.1 & 15.9 & 14.90 \\
\hline $7-3$ & 14.3 & 15.7 & 15.00 \\
\hline $7-2$ & 14.4 & 16.1 & 15.02 \\
\hline $7-1$ & 14.2 & 16.2 & 14.92 \\
\hline $8-4$ & 13.5 & 15.2 & 14.43 \\
\hline $8-3$ & 13.8 & 15.4 & 14.54 \\
\hline $8-2$ & 13.9 & 15.4 & 14.58 \\
\hline $8-1$ & 13.7 & 15.6 & 14.51 \\
\hline $10-4$ & 10.4 & 11.5 & 11.43 \\
\hline $10-3$ & 10.8 & 11.9 & 11.75 \\
\hline $10-2$ & 11.0 & 12.3 & 11.96 \\
\hline $10-1$ & 11.1 & 12.7 & 12.08 \\
\hline $12-4$ & 4.4 & 4.4 & 4.85 \\
\hline $12-3$ & 4.9 & 4.9 & 5.17 \\
\hline $12-2$ & 5.3 & 5.5 & 5.52 \\
\hline $12-1$ & 5.6 & 5.9 & 5.87 \\
\hline
\end{tabular}




\section{Appendix C Off-Axis Gamma Scans}

Inner Ring 1

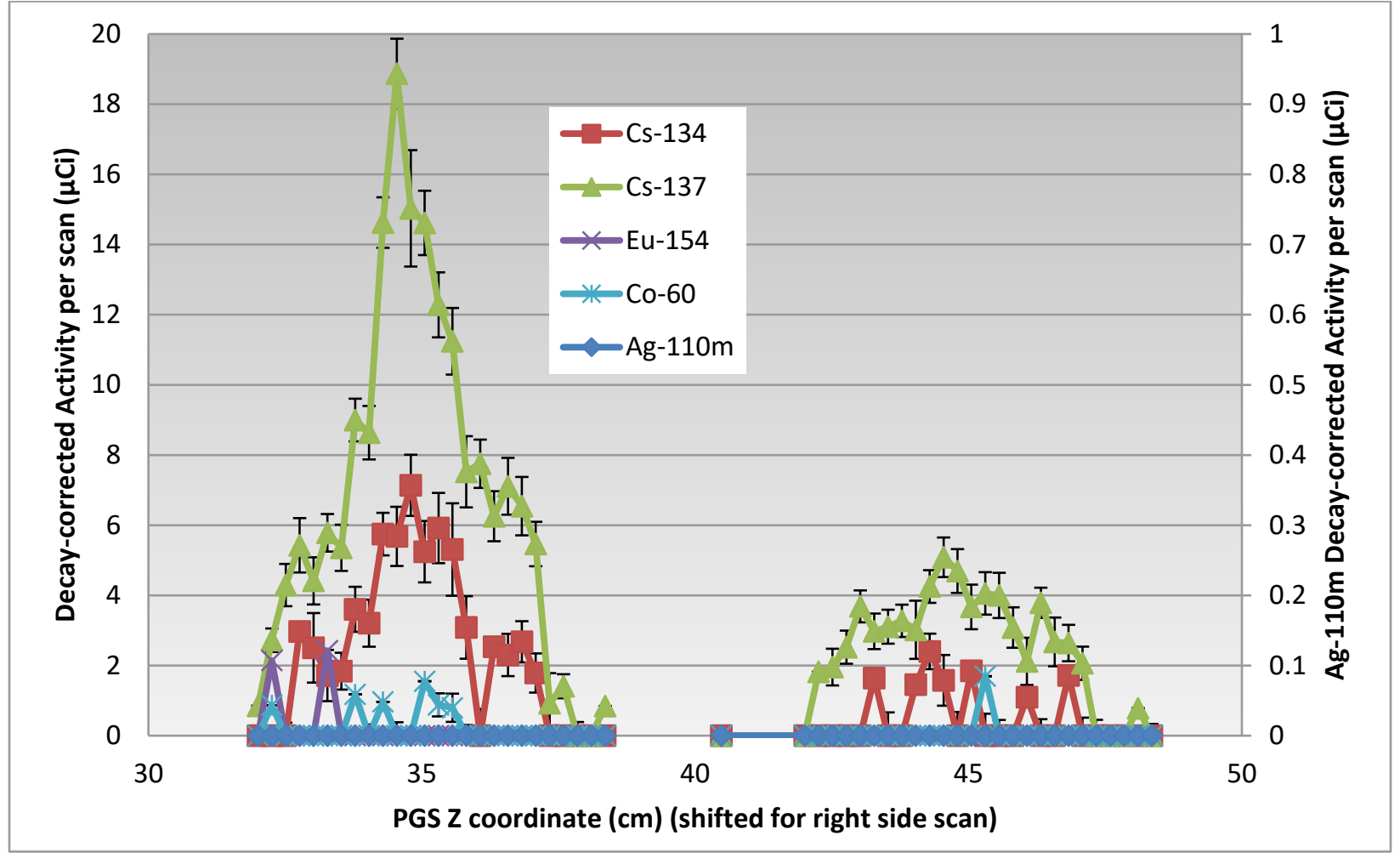

Outer Ring 1

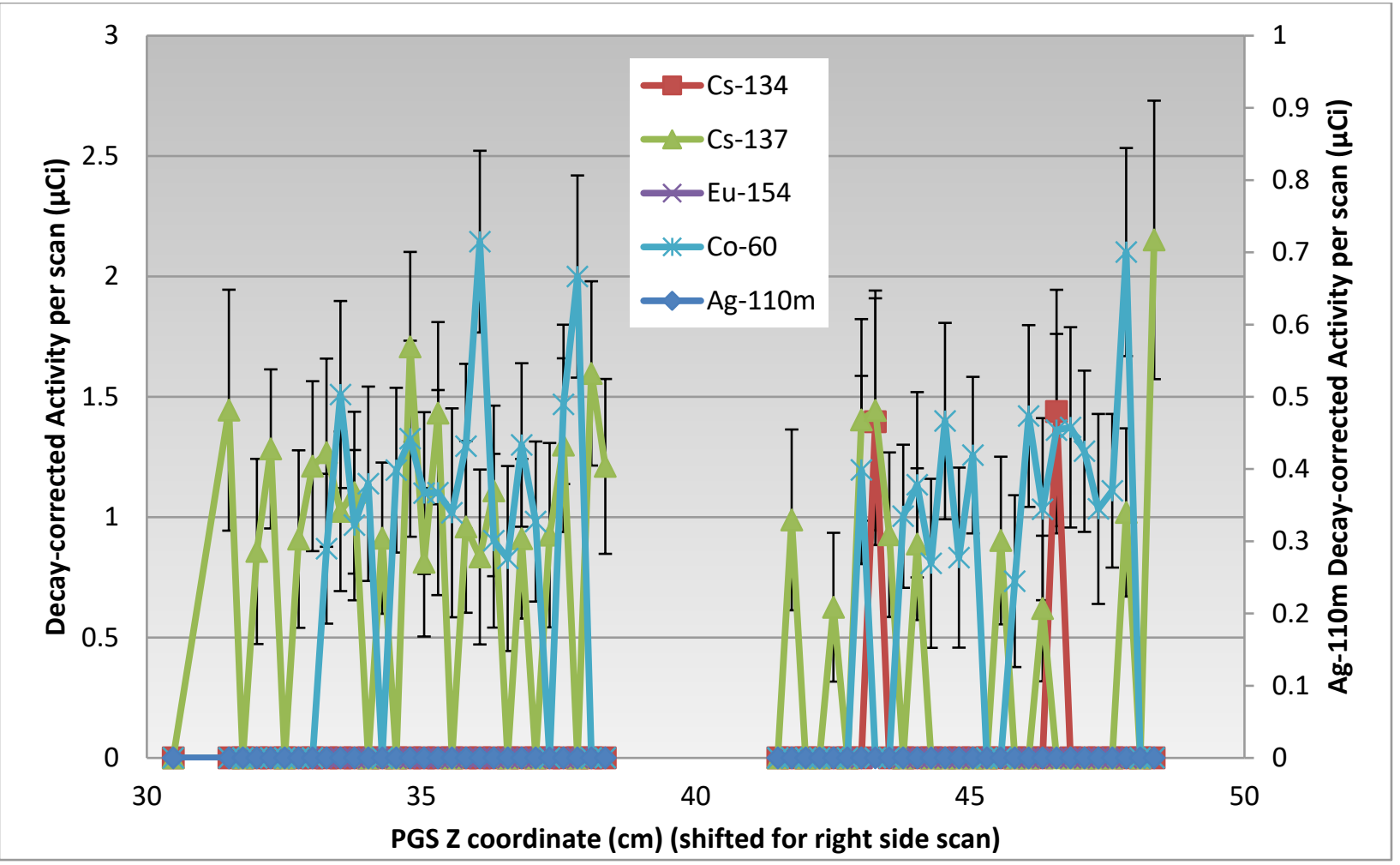


Inner Ring 3

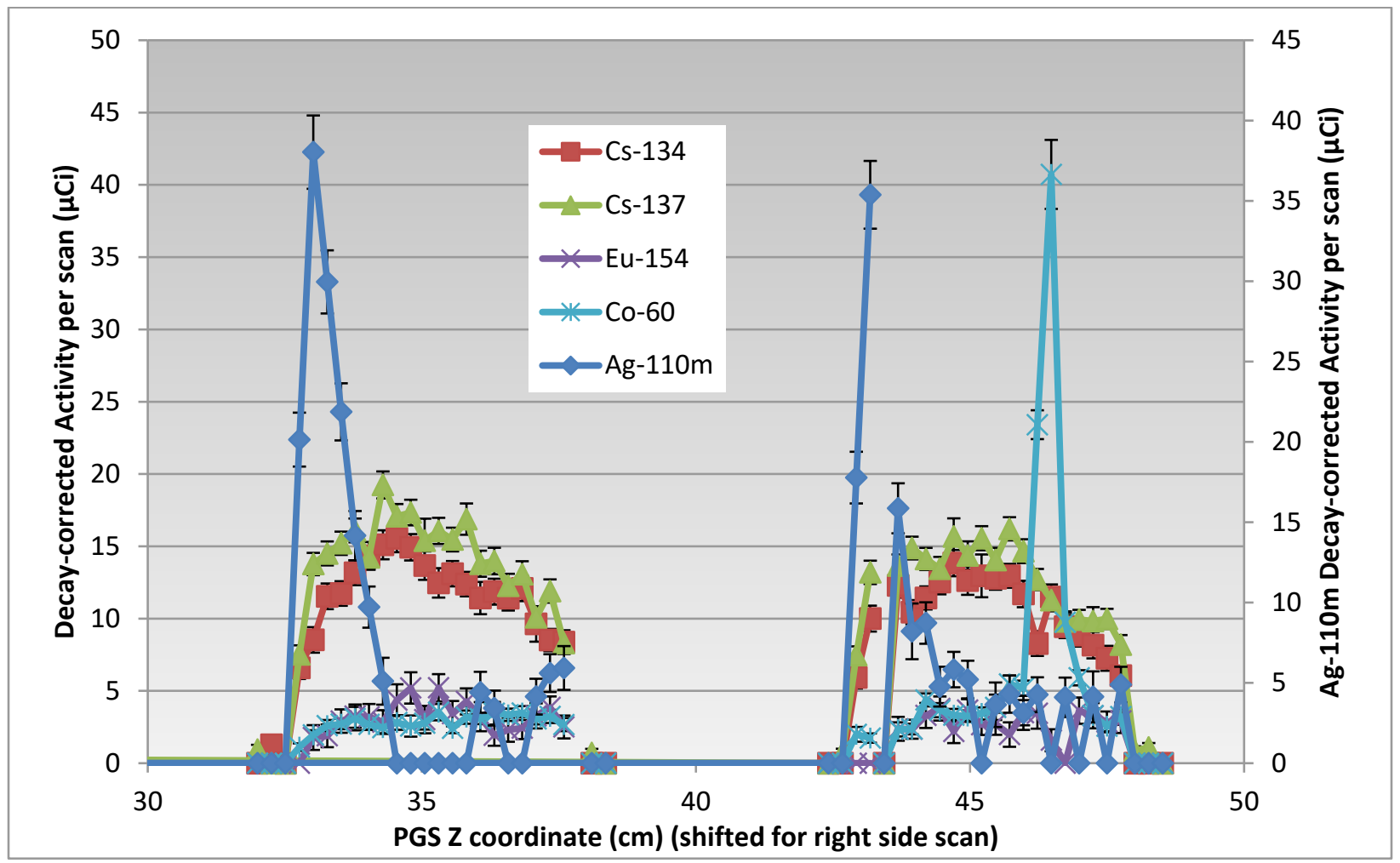

Outer Ring 3

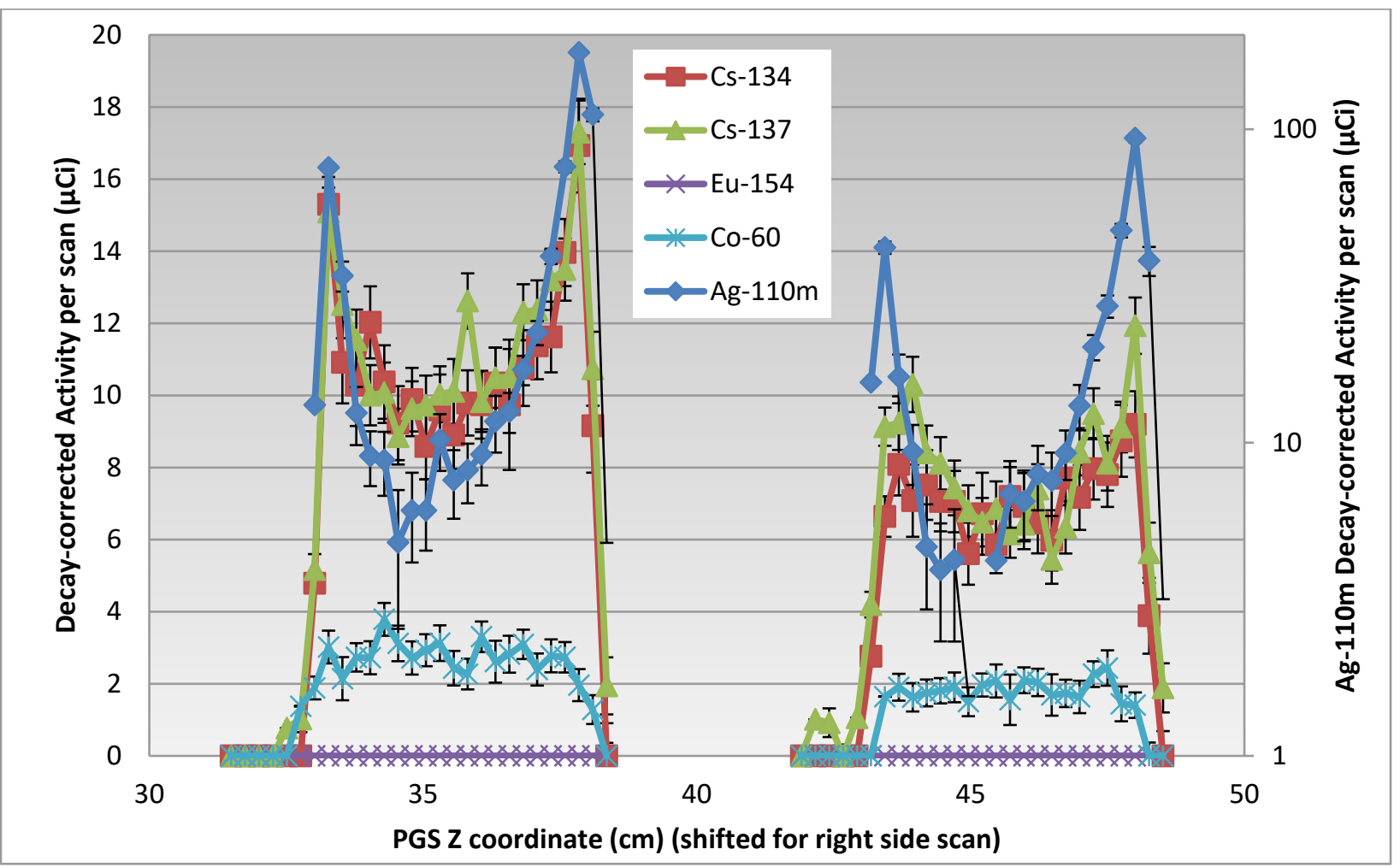


Inner Ring 4

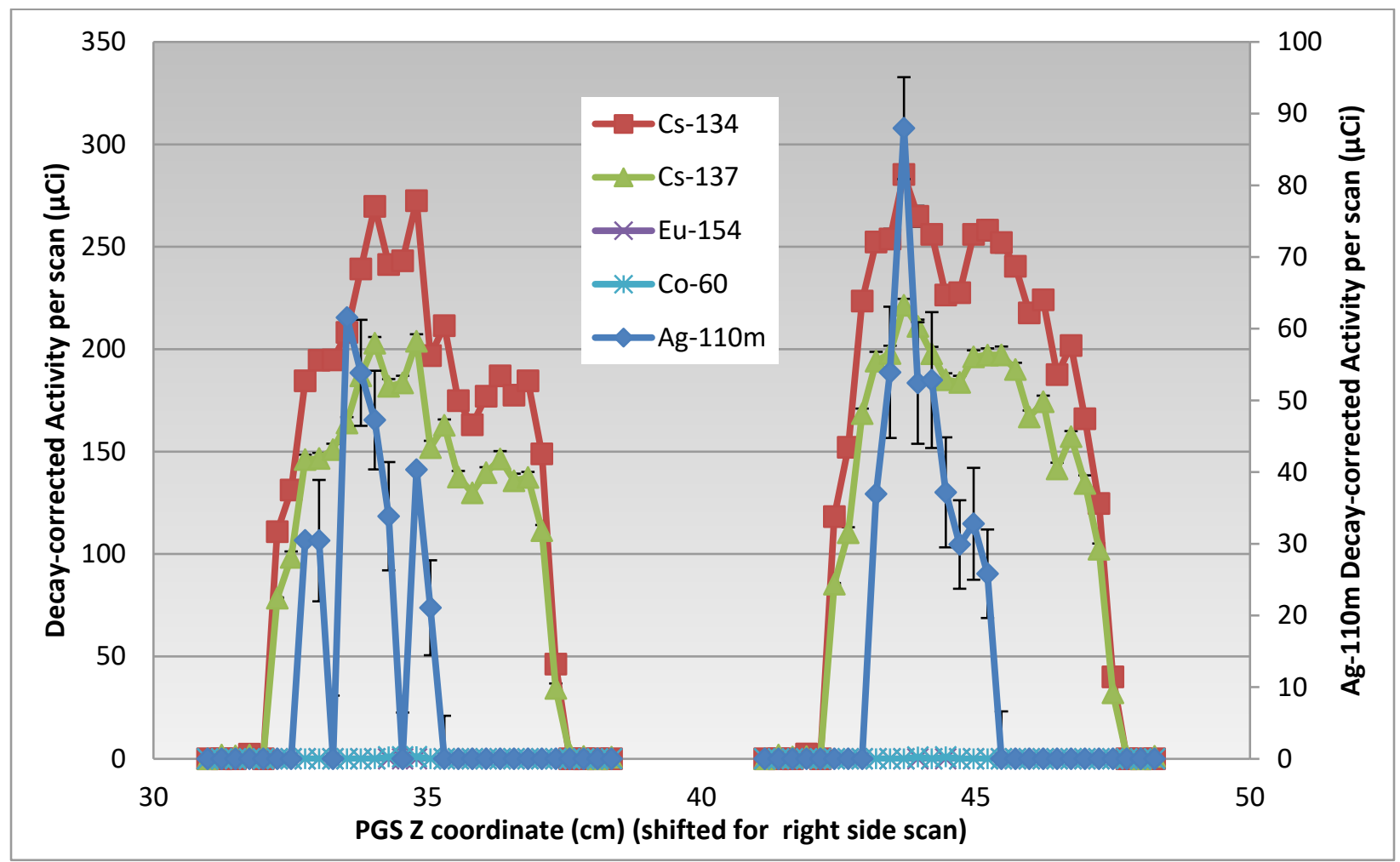

Outer Ring 4

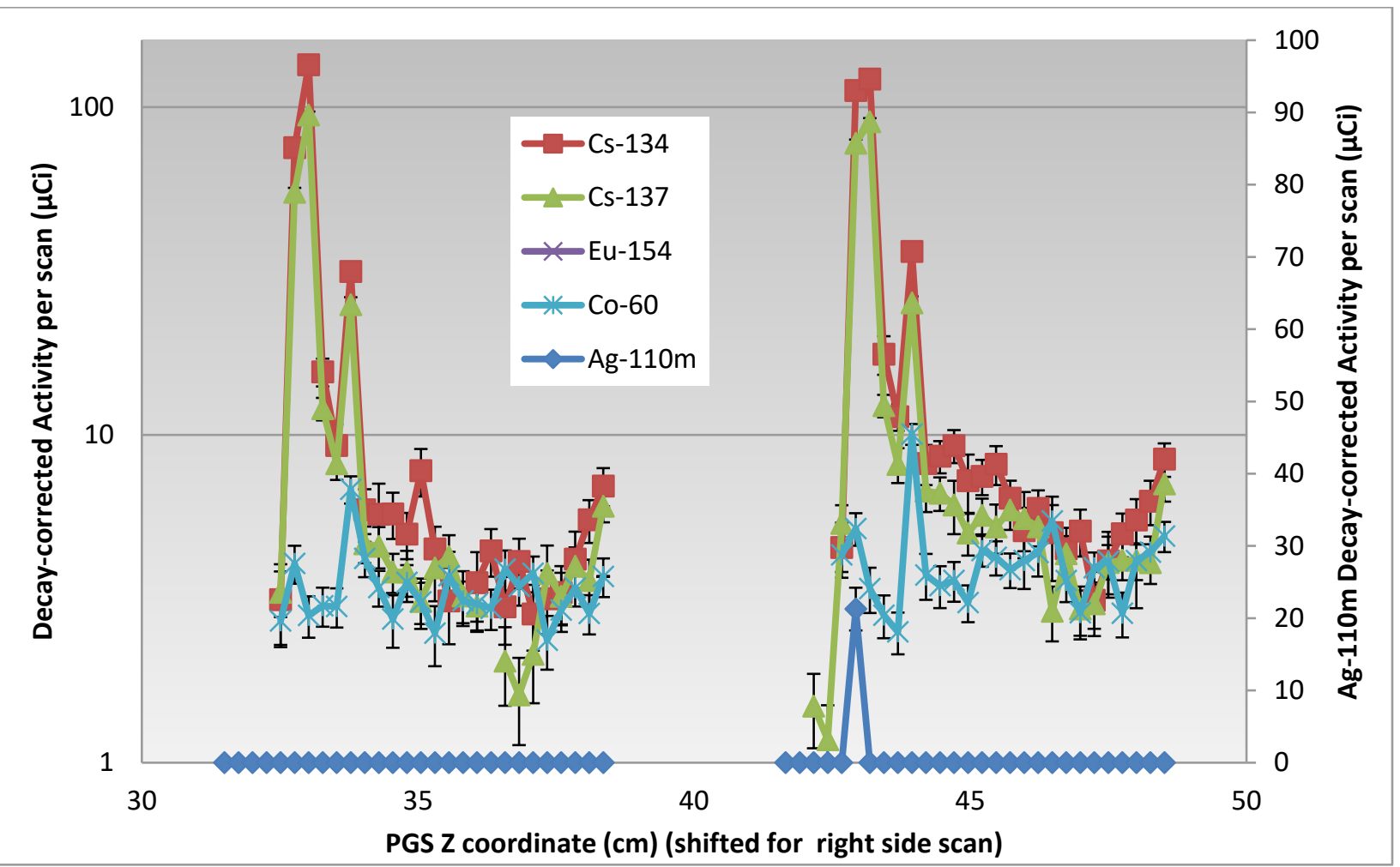


Inner Ring 5

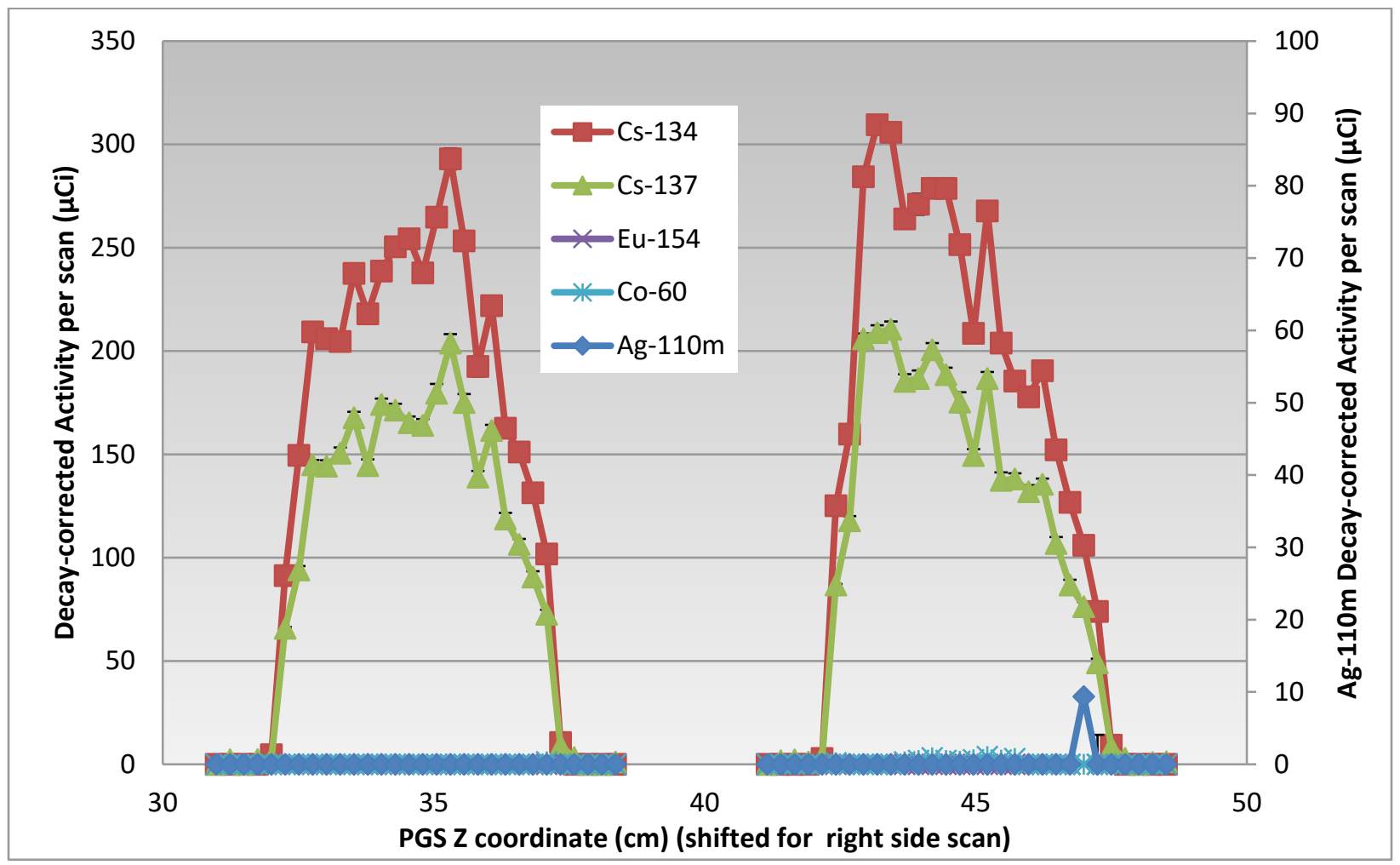

Outer Ring 5

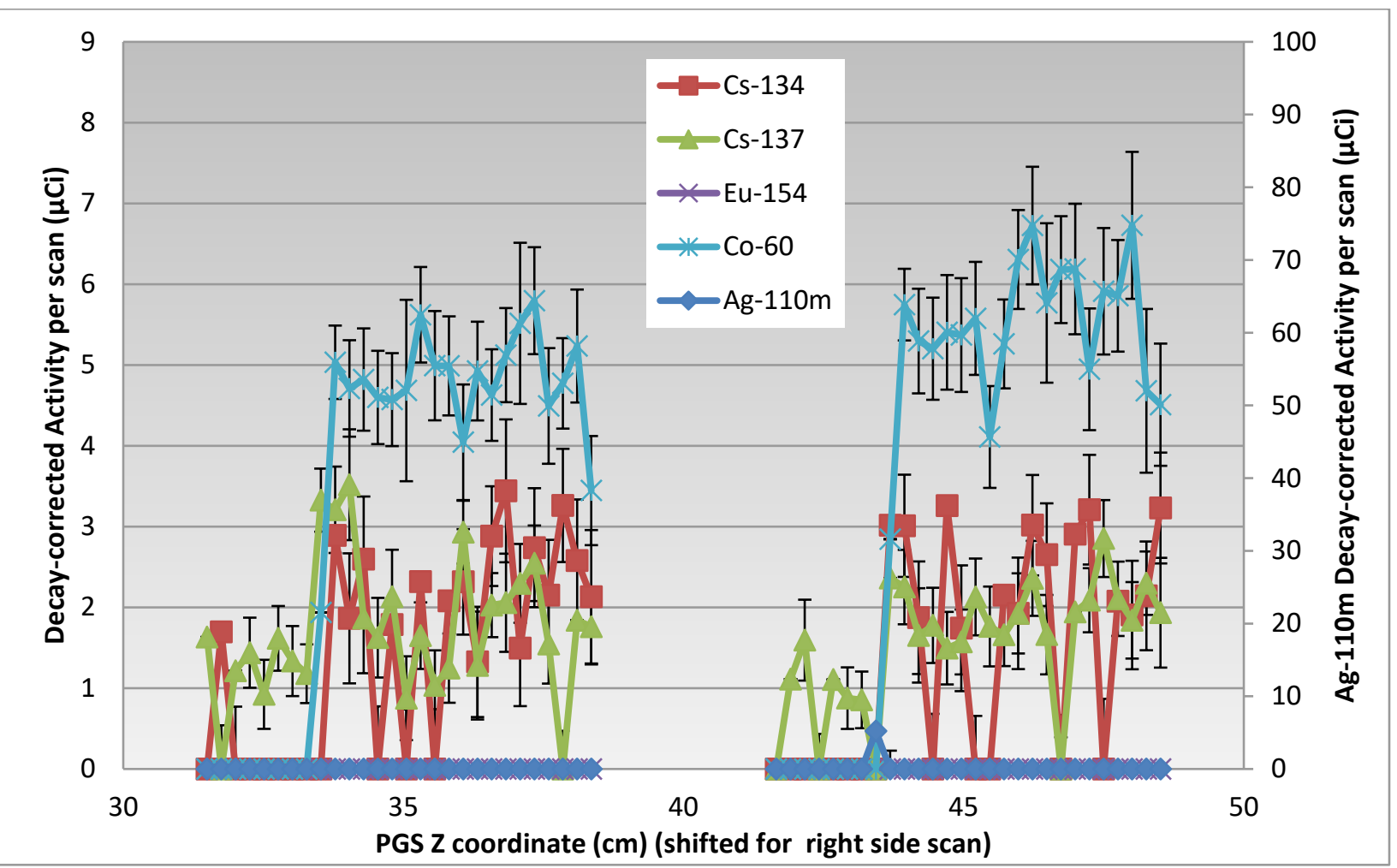


Inner Ring 7

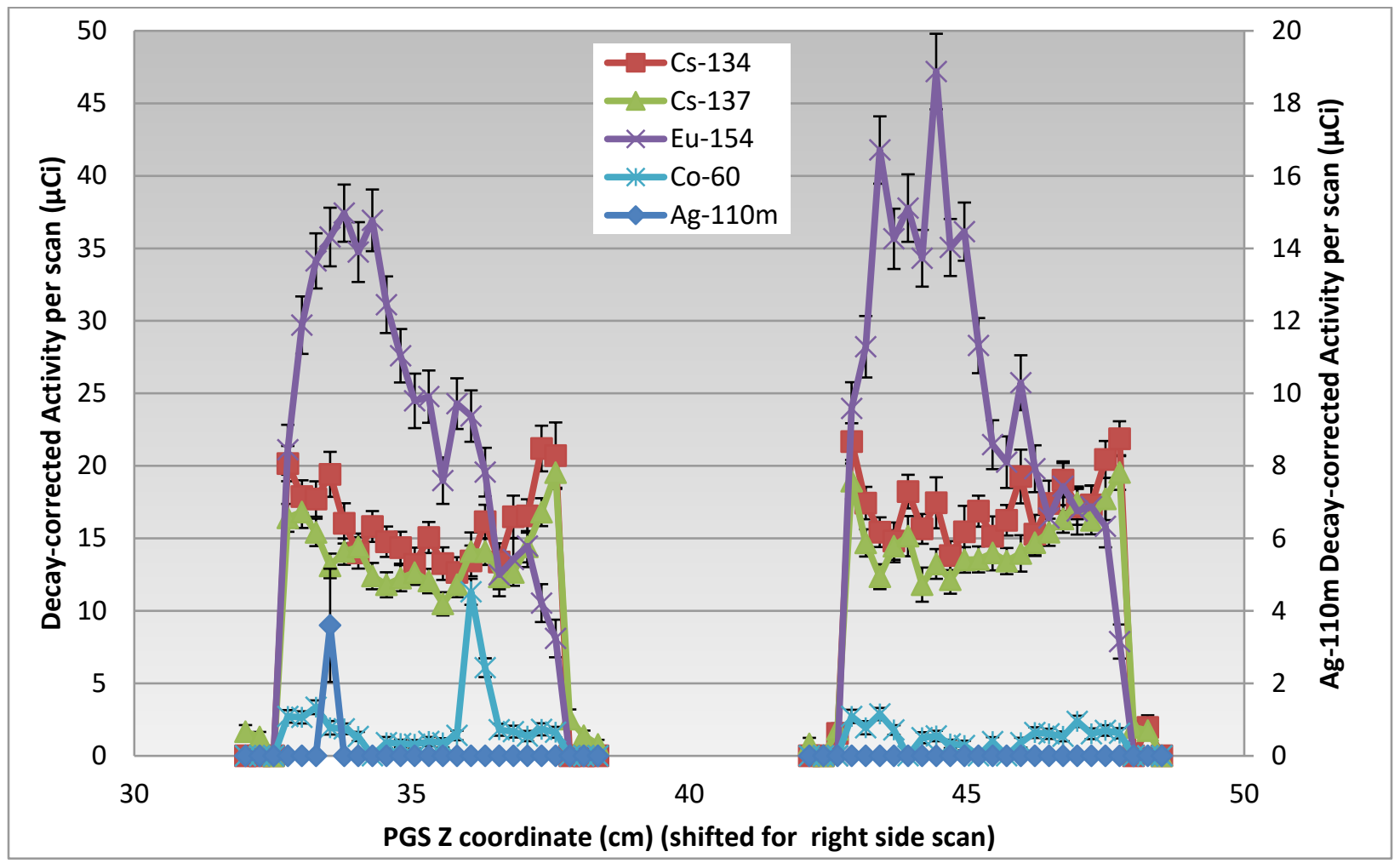

Outer Ring 7

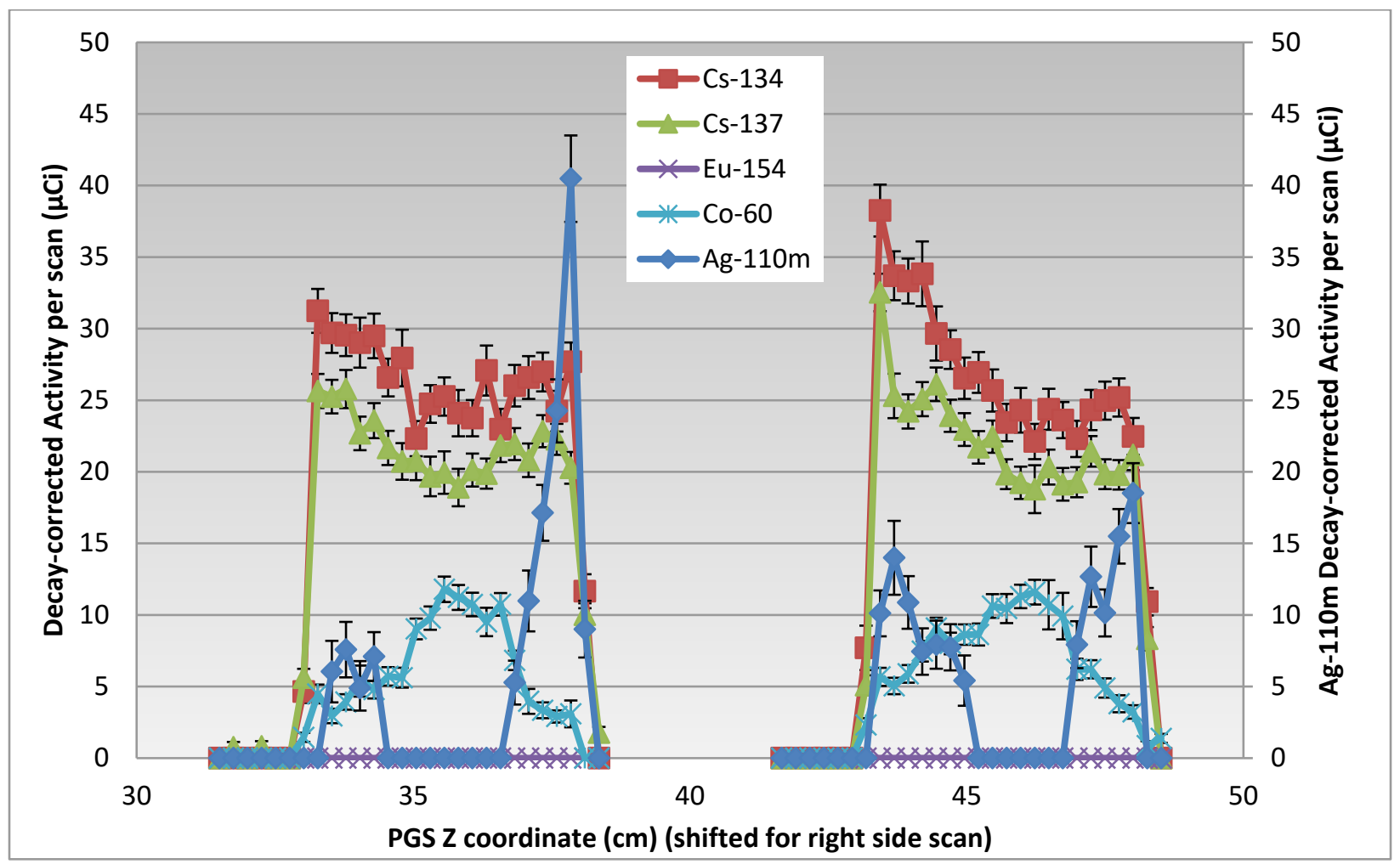


Inner Ring 8

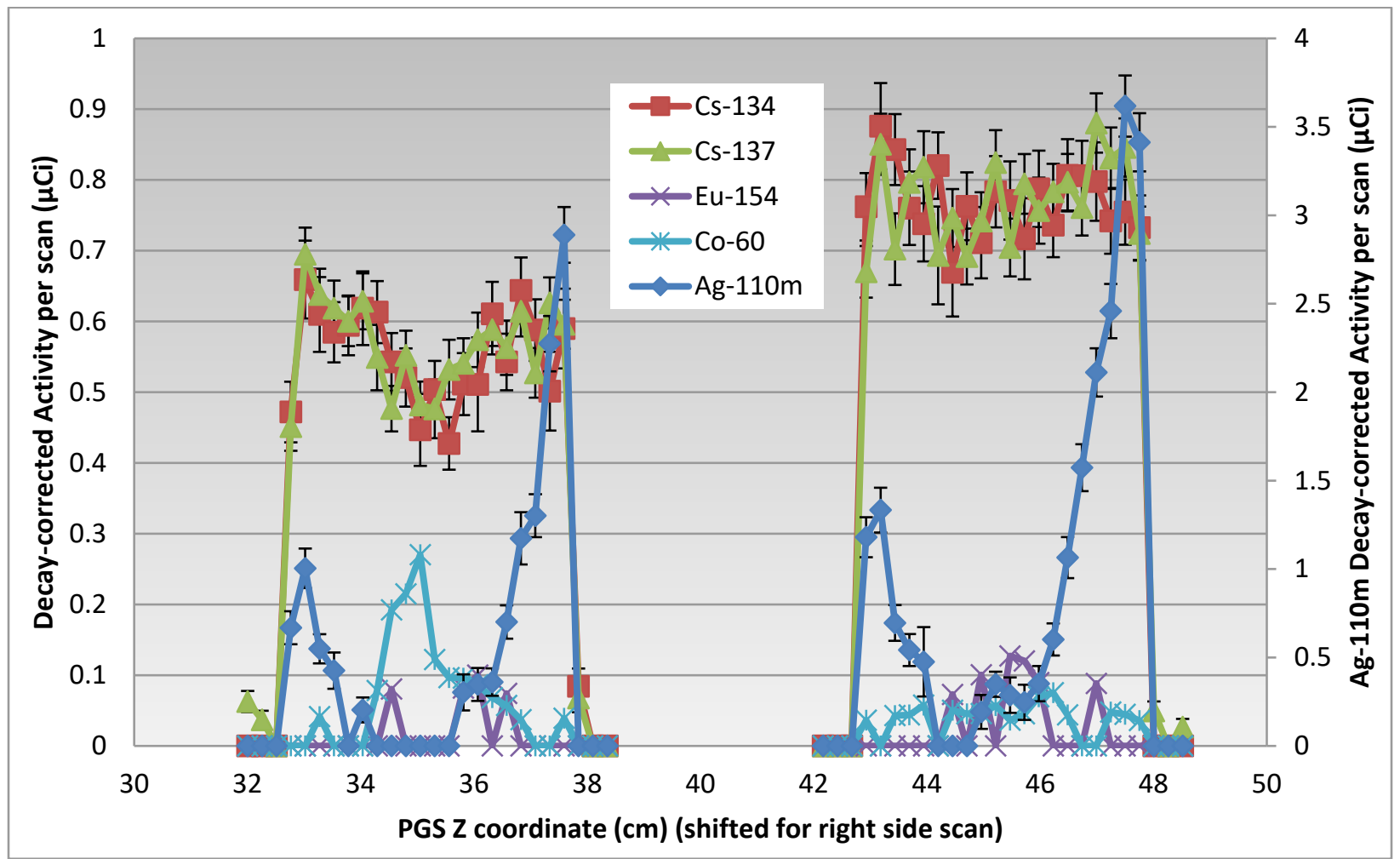

Outer Ring 8

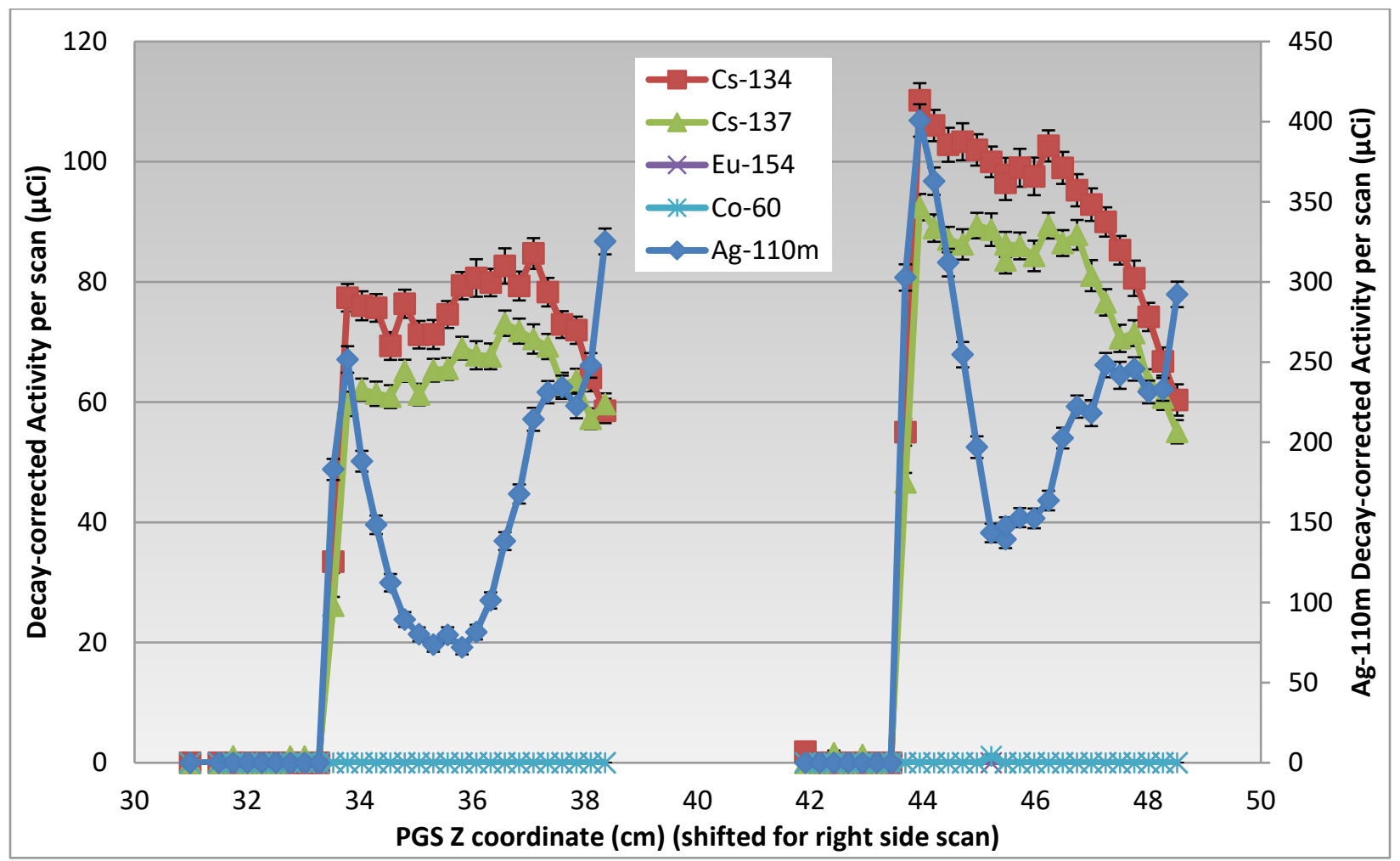


Inner Ring 10

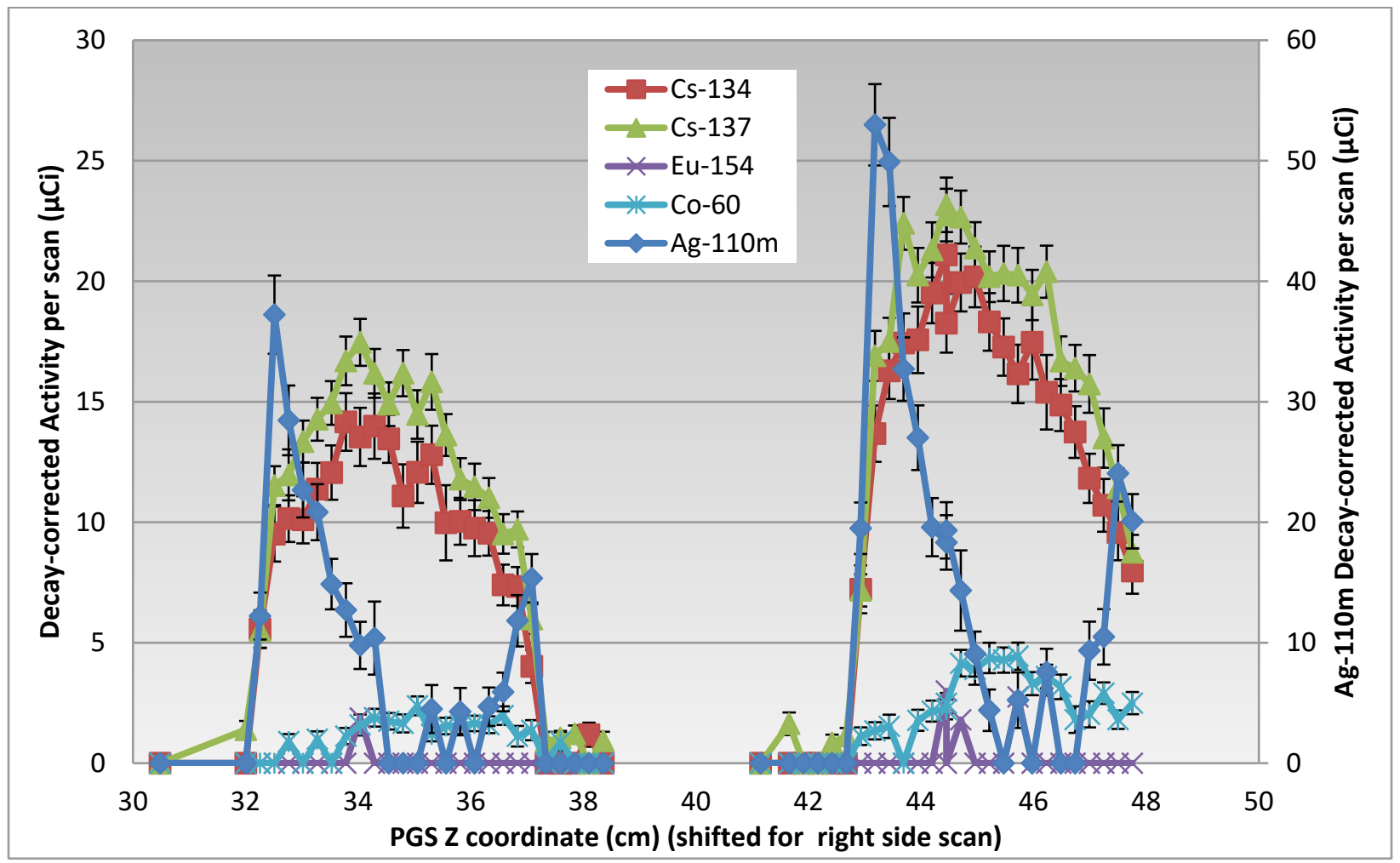

Outer Ring 10

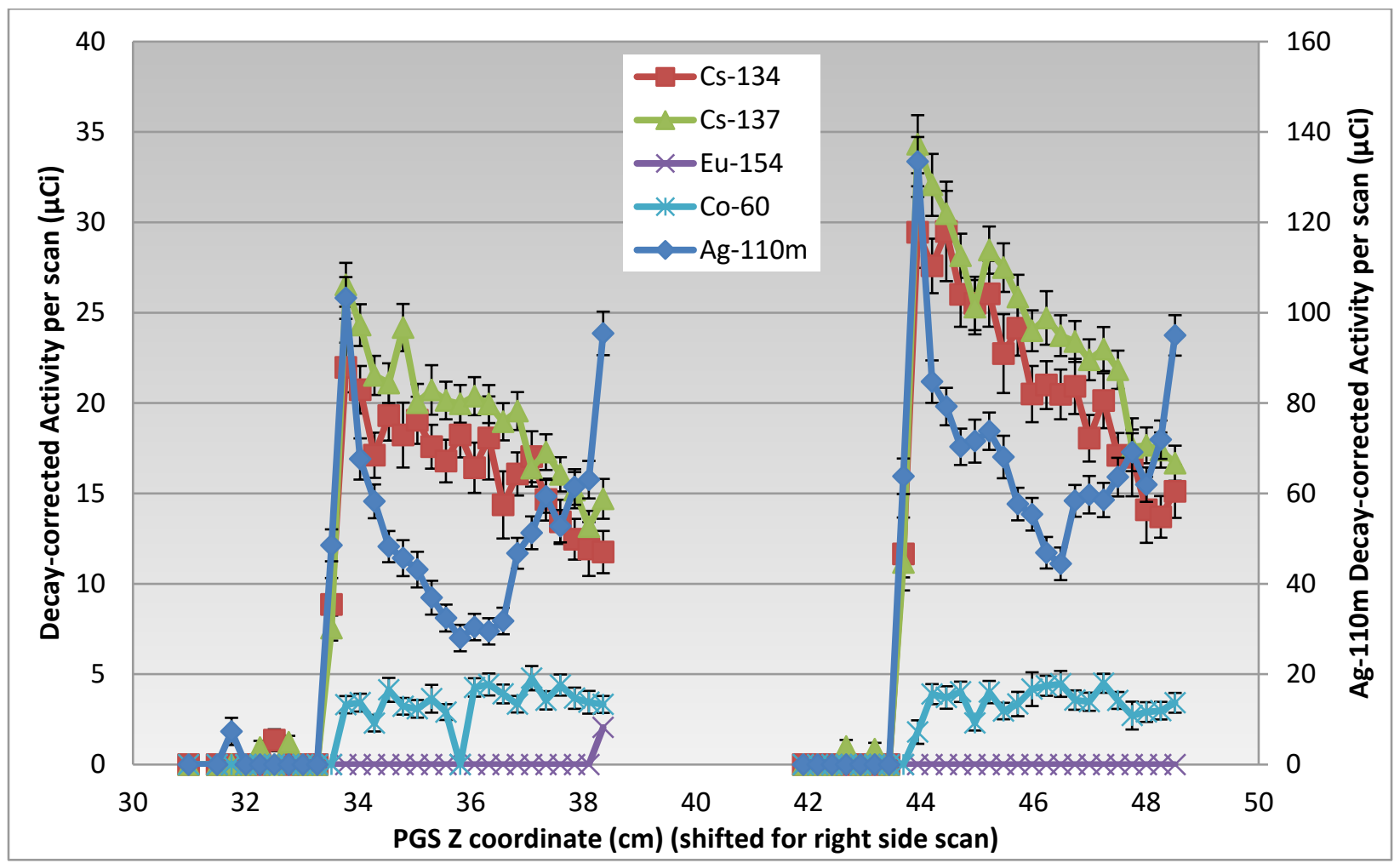


Inner Ring 12

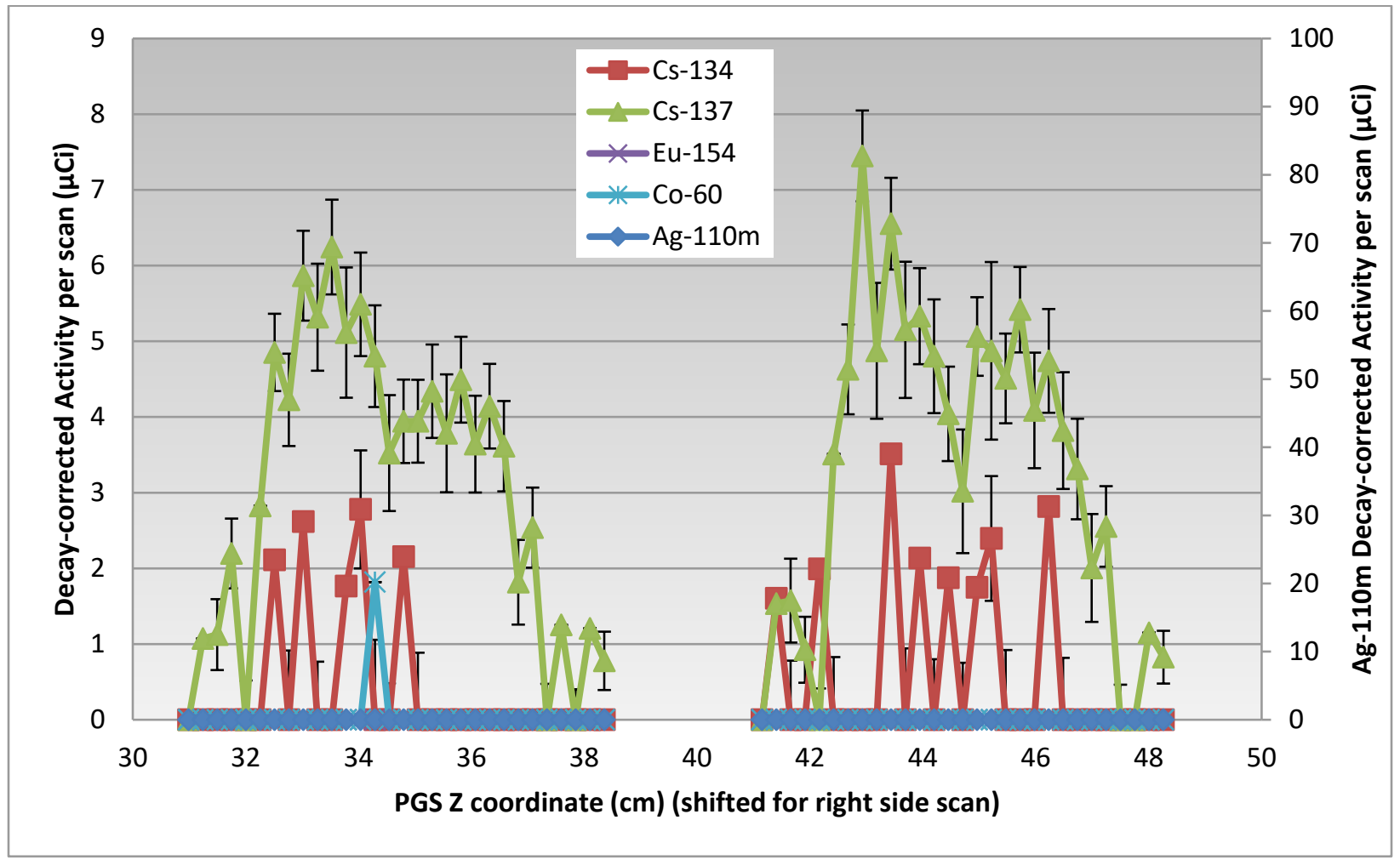

Outer Ring 12

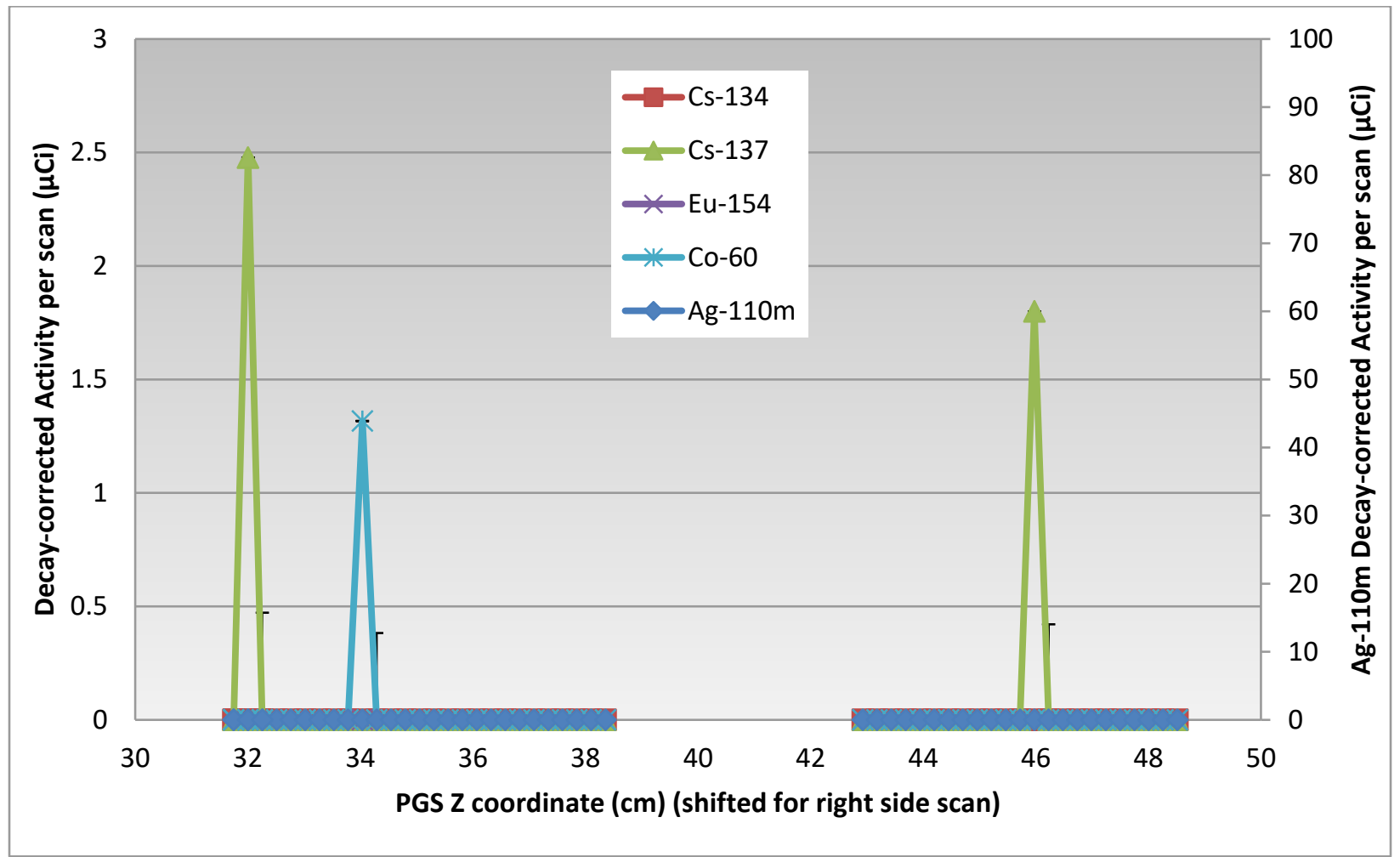




\section{Appendix D Gamma Tomography Data}

The distance between each scan was always 0.050 inches $(0.127 \mathrm{~cm})$.

\section{Inner Ring 3 Axial Middle}

Angles for MID IR-03 Scans

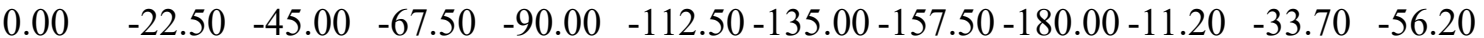

Cs-137 Input R matrix

\begin{tabular}{|c|c|c|c|c|c|c|c|c|c|c|c|}
\hline 0.00 & 2.53 & 1.97 & 2.60 & 3.35 & 2.11 & 0.00 & 0.00 & 1.44 & 1.78 & 3.37 & 2.98 \\
\hline 5.47 & 8.13 & 7.62 & 5.94 & 5.65 & 4.48 & 3.01 & 0.00 & 0.00 & 6.20 & 5.61 & 6.89 \\
\hline 9.65 & 9.76 & 12.47 & 11.47 & 9.60 & 9.88 & 9.30 & 8.01 & 6.42 & 8.52 & 10.27 & 10.84 \\
\hline 14.88 & 14.76 & 15.95 & 14.10 & 17.04 & 14.47 & 14.57 & 10.94 & 8.82 & 13.20 & 13.69 & 14.19 \\
\hline 19.05 & 19.72 & 20.81 & 22.87 & 20.74 & 19.99 & 20.32 & 17.35 & 15.43 & 19.45 & 20.49 & 20.15 \\
\hline 18.76 & 17.86 & 18.30 & 14.98 & 16.49 & 18.73 & 21.12 & 25.73 & 23.53 & 15.03 & 18.83 & 19.57 \\
\hline 15.15 & 11.83 & 13.28 & 12.41 & 12.46 & 15.22 & 14.18 & 16.03 & 15.56 & 14.64 & 13.63 & 12.63 \\
\hline 13.32 & 12.79 & 12.51 & 12.26 & 13.33 & 12.67 & 12.88 & 12.80 & 11.51 & 11.08 & 13.57 & 14.52 \\
\hline 14.01 & 13.08 & 11.47 & 14.70 & 13.00 & 9.66 & 14.19 & 11.25 & 10.63 & 12.98 & 10.50 & 12.03 \\
\hline 11.75 & 11.30 & 13.55 & 13.21 & 10.83 & 10.43 & 11.79 & 11.38 & 13.10 & 9.72 & 11.87 & 11.96 \\
\hline 11.28 & 10.81 & 11.94 & 11.02 & 14.72 & 10.31 & 13.64 & 12.15 & 11.55 & 13.57 & 12.91 & 11.34 \\
\hline 12.72 & 10.42 & 12.74 & 12.42 & 12.23 & 11.83 & 11.91 & 12.33 & 10.42 & 12.29 & 10.70 & 12.37 \\
\hline 13.02 & 10.15 & 13.36 & 15.11 & 12.43 & 13.82 & 13.49 & 15.03 & 13.41 & 10.67 & 13.29 & 13.46 \\
\hline 14.57 & 13.89 & 15.40 & 16.55 & 14.49 & 14.20 & 11.18 & 11.97 & 11.30 & 15.26 & 16.81 & 16.59 \\
\hline 18.60 & 19.43 & 20.30 & 20.49 & 24.54 & 21.30 & 17.29 & 14.80 & 12.89 & 20.02 & 17.36 & 19.71 \\
\hline 20.73 & 19.88 & 20.08 & 18.07 & 19.16 & 21.38 & 20.20 & 17.33 & 17.34 & 20.62 & 18.60 & 17.03 \\
\hline 17.33 & 13.22 & 13.96 & 15.24 & 14.83 & 14.90 & 15.82 & 19.77 & 19.30 & 12.83 & 10.08 & 9.87 \\
\hline 8.48 & 7.60 & 9.88 & 7.79 & 9.50 & 9.21 & 10.86 & 12.86 & 14.70 & 6.50 & 7.29 & 8.73 \\
\hline 4.24 & 3.66 & 3.28 & 3.53 & 2.63 & 5.12 & 5.59 & 6.64 & 10.68 & 5.37 & 3.60 & 3.09 \\
\hline 0.00 & 0.00 & 1.27 & 0.00 & 0.00 & 1.80 & 1.79 & 2.03 & 8.45 & 0.00 & 0.00 & 0.00 \\
\hline 0.00 & 0.00 & 0.00 & 0.00 & 0.00 & 0.00 & 0.00 & 0.00 & 2.08 & 0.00 & 0.00 & 0.00 \\
\hline 0.00 & 0.00 & 0.00 & 0.00 & 0.00 & 0.00 & 0.00 & 0.00 & 0.00 & 0.00 & 0.00 & 0.00 \\
\hline 0.00 & 1.46 & 0.00 & 0.00 & 0.00 & 0.00 & 0.00 & 0.00 & 0.00 & 0.00 & 0.00 & 0.00 \\
\hline
\end{tabular}




\section{Cs-134 Input R matrix}

\begin{tabular}{|c|c|c|c|c|c|c|c|c|c|c|c|}
\hline 0.00 & 2.98 & 2.23 & 0.00 & 3.11 & 0.00 & 0.00 & 0.00 & 0.00 & 0.00 & 4.50 & 2.70 \\
\hline 4.57 & 4.96 & 7.05 & 6.74 & 6.04 & 3.79 & 4.73 & 0.00 & 0.00 & 4.80 & 7.28 & 7.29 \\
\hline 9.08 & 7.68 & 12.76 & 9.76 & 11.83 & 9.06 & 7.72 & 6.22 & 4.71 & 8.34 & 8.82 & 10.90 \\
\hline 10.86 & 13.88 & 14.78 & 16.23 & 11.16 & 15.46 & 12.66 & 9.82 & 6.94 & 16.36 & 14.28 & 13.21 \\
\hline 19.32 & 19.86 & 17.23 & 17.80 & 18.08 & 18.00 & 17.58 & 13.36 & 14.50 & 17.98 & 19.09 & 20.05 \\
\hline 15.12 & 15.16 & 14.05 & 13.12 & 14.93 & 19.95 & 20.57 & 18.15 & 16.75 & 17.74 & 13.08 & 15.66 \\
\hline 15.83 & 12.20 & 10.73 & 11.93 & 12.36 & 10.42 & 12.36 & 14.81 & 20.28 & 12.25 & 12.17 & 11.51 \\
\hline 12.24 & 10.63 & 9.72 & 11.91 & 10.55 & 10.91 & 12.93 & 11.19 & 11.87 & 10.52 & 12.94 & 11.17 \\
\hline 10.89 & 9.85 & 10.04 & 11.92 & 10.90 & 9.83 & 10.72 & 13.01 & 11.59 & 9.31 & 12.76 & 10.31 \\
\hline 10.20 & 8.09 & 9.60 & 12.02 & 10.90 & 8.23 & 8.46 & 9.61 & 8.98 & 9.07 & 9.14 & 10.29 \\
\hline 12.09 & 11.46 & 11.26 & 10.66 & 9.26 & 7.93 & 7.31 & 8.74 & 11.50 & 11.30 & 11.35 & 8.56 \\
\hline 12.40 & 13.16 & 13.42 & 10.27 & 9.81 & 12.32 & 10.38 & 10.38 & 18.39 & 11.04 & 10.34 & 12.28 \\
\hline 9.53 & 12.97 & 13.01 & 11.55 & 11.80 & 11.79 & 11.72 & 13.20 & 12.03 & 11.48 & 14.70 & 11.45 \\
\hline 12.44 & 14.77 & 14.23 & 12.97 & 15.98 & 12.75 & 13.24 & 10.89 & 11.81 & 13.38 & 14.38 & 14.02 \\
\hline 20.39 & 19.77 & 16.21 & 18.50 & 19.22 & 19.37 & 13.67 & 15.19 & 12.57 & 18.28 & 15.90 & 15.77 \\
\hline 15.58 & 17.19 & 14.41 & 16.94 & 18.50 & 22.05 & 16.96 & 17.80 & 14.00 & 16.86 & 12.85 & 18.41 \\
\hline 15.70 & 11.92 & 10.11 & 13.07 & 13.62 & 11.06 & 14.78 & 13.84 & 19.37 & 15.40 & 10.82 & 11.44 \\
\hline 8.84 & 6.08 & 6.19 & 2.37 & 9.11 & 5.43 & 10.16 & 12.28 & 12.38 & 5.89 & 6.36 & 6.00 \\
\hline 4.40 & 3.91 & 2.51 & 5.64 & 3.31 & 3.93 & 5.58 & 5.60 & 6.64 & 0.00 & 0.00 & 4.95 \\
\hline 0.00 & 0.00 & 0.00 & 0.00 & 0.00 & 0.00 & 0.00 & 3.18 & 3.78 & 0.00 & 0.00 & 0.00 \\
\hline 0.00 & 0.00 & 0.00 & 0.00 & 0.00 & 0.00 & 0.00 & 0.00 & 0.00 & 0.00 & 0.00 & 0.00 \\
\hline 0.00 & 0.00 & 0.00 & 0.00 & 0.00 & 0.00 & 0.00 & 0.00 & 0.00 & 0.00 & 0.00 & 0.00 \\
\hline 0.00 & 0.00 & 0.00 & 0.00 & 0.00 & 0.00 & 0.00 & 0.00 & 0.00 & 0.00 & 0.00 & 0.00 \\
\hline
\end{tabular}




\section{Co-60 Input R matrix}

\begin{tabular}{|c|c|c|c|c|c|c|c|c|c|c|c|}
\hline 0.00 & 0.00 & 0.00 & 0.00 & 0.00 & 0.00 & 0.00 & 0.00 & 0.00 & 0.00 & 0.00 & 0.00 \\
\hline 0.00 & 11.52 & 31.02 & 0.00 & 0.00 & 0.00 & 0.00 & 0.00 & 0.00 & 2.02 & 27.73 & 4.32 \\
\hline 3.13 & 44.17 & 26.15 & 21.34 & 0.00 & 2.26 & 2.04 & 0.00 & 0.00 & 39.90 & 30.92 & 49.83 \\
\hline 40.48 & 3.98 & 2.31 & 44.25 & 3.99 & 1.96 & 0.00 & 0.00 & 2.83 & 17.96 & 3.06 & 9.13 \\
\hline 8.68 & 3.57 & 2.87 & 5.80 & 10.51 & 2.66 & 3.90 & 0.00 & 2.79 & 3.72 & 3.32 & 4.06 \\
\hline 4.01 & 2.75 & 0.00 & 2.52 & 47.03 & 3.01 & 3.64 & 5.42 & 4.45 & 4.29 & 3.18 & 3.74 \\
\hline 3.24 & 2.44 & 2.20 & 0.00 & 4.01 & 0.00 & 2.30 & 3.77 & 4.98 & 3.11 & 3.19 & 3.37 \\
\hline 3.15 & 2.17 & 2.33 & 0.00 & 2.18 & 6.13 & 3.06 & 3.67 & 0.00 & 2.81 & 0.00 & 2.77 \\
\hline 3.71 & 0.00 & 0.00 & 0.00 & 0.00 & 56.65 & 2.46 & 3.04 & 2.16 & 2.13 & 0.00 & 0.00 \\
\hline 2.51 & 2.96 & 2.66 & 0.00 & 0.00 & 3.72 & 0.00 & 2.90 & 0.00 & 2.28 & 2.85 & 2.16 \\
\hline 0.00 & 1.82 & 3.23 & 2.17 & 0.00 & 0.00 & 4.93 & 0.00 & 0.00 & 3.62 & 2.35 & 0.00 \\
\hline 3.23 & 2.83 & 2.06 & 1.96 & 0.00 & 2.81 & 56.29 & 3.11 & 2.75 & 2.98 & 0.00 & 2.71 \\
\hline 0.00 & 3.81 & 2.49 & 3.00 & 2.48 & 2.45 & 2.84 & 0.00 & 0.00 & 2.48 & 2.63 & 2.69 \\
\hline 3.08 & 2.52 & 0.00 & 2.57 & 3.48 & 2.63 & 2.33 & 2.87 & 2.88 & 2.90 & 2.05 & 0.00 \\
\hline 4.34 & 0.00 & 4.42 & 4.86 & 5.10 & 3.12 & 3.57 & 56.68 & 3.77 & 3.88 & 0.00 & 5.33 \\
\hline 3.71 & 2.97 & 0.00 & 2.29 & 2.99 & 3.77 & 3.06 & 10.18 & 4.12 & 2.82 & 2.34 & 1.93 \\
\hline 0.00 & 0.00 & 2.11 & 0.00 & 2.40 & 0.00 & 0.00 & 3.23 & 21.72 & 2.54 & 0.00 & 0.00 \\
\hline 0.00 & 0.00 & 2.16 & 2.23 & 0.00 & 0.00 & 3.01 & 0.00 & 40.37 & 2.73 & 0.00 & 0.00 \\
\hline 0.00 & 0.00 & 0.00 & 0.00 & 0.00 & 0.00 & 0.00 & 0.00 & 2.21 & 0.00 & 0.00 & 0.00 \\
\hline 0.00 & 0.00 & 0.00 & 0.00 & 0.00 & 0.00 & 0.00 & 0.00 & 0.00 & 0.00 & 0.00 & 0.00 \\
\hline 0.00 & 0.00 & 0.00 & 0.00 & 0.00 & 0.00 & 0.00 & 0.00 & 0.00 & 0.00 & 0.00 & 0.00 \\
\hline 0.00 & 0.00 & 0.00 & 0.00 & 0.00 & 0.00 & 0.00 & 0.00 & 0.00 & 0.00 & 0.00 & 0.00 \\
\hline 0.00 & 0.00 & 0.00 & 0.00 & 0.00 & 0.00 & 0.00 & 0.00 & 0.00 & 0.00 & 0.00 & 0.00 \\
\hline
\end{tabular}




\section{Inner Ring 3 Axial Top}

Angles for Upper IR-03 Scan

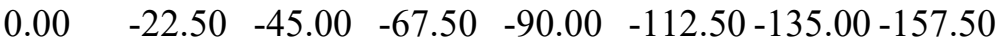

Cs-137 Input R matrix

\begin{tabular}{|c|c|c|c|c|c|c|c|}
\hline 0.00 & 0.00 & 0.00 & 0.00 & 0.00 & 0.00 & 0.00 & 0.00 \\
\hline 0.00 & 2.00 & 3.24 & 1.71 & 0.00 & 0.00 & 0.00 & 0.00 \\
\hline 5.70 & 5.54 & 6.33 & 4.11 & 5.74 & 0.00 & 2.14 & 2.32 \\
\hline 11.18 & 10.34 & 8.97 & 10.19 & 8.87 & 8.70 & 8.66 & 4.47 \\
\hline 13.75 & 13.50 & 11.08 & 14.77 & 15.18 & 12.84 & 11.41 & 9.21 \\
\hline 15.33 & 23.85 & 18.80 & 20.89 & 23.08 & 17.63 & 18.30 & 16.86 \\
\hline 16.56 & 17.56 & 16.89 & 18.12 & 18.92 & 19.69 & 19.72 & 16.25 \\
\hline 13.05 & 13.42 & 13.81 & 10.99 & 12.66 & 15.30 & 15.60 & 15.23 \\
\hline 10.31 & 13.11 & 13.92 & 10.21 & 11.37 & 12.98 & 13.12 & 12.13 \\
\hline 10.20 & 10.66 & 9.98 & 12.60 & 11.62 & 12.83 & 14.17 & 12.75 \\
\hline 10.84 & 10.93 & 10.34 & 10.17 & 13.15 & 11.72 & 11.77 & 9.65 \\
\hline 13.21 & 10.99 & 10.11 & 11.44 & 11.48 & 7.87 & 12.42 & 11.20 \\
\hline 11.37 & 8.66 & 13.16 & 9.72 & 13.37 & 10.34 & 10.61 & 11.17 \\
\hline 13.83 & 12.04 & 10.15 & 12.31 & 11.25 & 11.91 & 8.55 & 9.87 \\
\hline 9.61 & 12.53 & 15.28 & 15.89 & 12.98 & 9.60 & 11.19 & 11.25 \\
\hline 16.91 & 21.01 & 18.78 & 19.03 & 18.04 & 16.98 & 15.41 & 11.01 \\
\hline 21.25 & 18.13 & 20.27 & 19.56 & 18.81 & 20.34 & 18.03 & 21.63 \\
\hline 16.18 & 10.75 & 16.20 & 12.73 & 14.80 & 16.55 & 14.77 & 17.28 \\
\hline 9.52 & 6.39 & 9.10 & 10.22 & 10.06 & 11.07 & 11.77 & 12.78 \\
\hline 7.11 & 2.98 & 2.94 & 5.12 & 5.46 & 5.19 & 7.16 & 8.64 \\
\hline 0.00 & 0.00 & 0.00 & 1.89 & 0.00 & 1.41 & 3.67 & 5.14 \\
\hline 0.00 & 0.00 & 0.00 & 0.00 & 0.00 & 0.00 & 0.00 & 0.00 \\
\hline 0.00 & 0.00 & 0.00 & 0.00 & 0.00 & 0.00 & 0.00 & 0.00 \\
\hline
\end{tabular}




\section{Cs-134 Input R matrix}

\begin{tabular}{|c|c|c|c|c|c|c|c|}
\hline 0.00 & 0.00 & 0.00 & 0.00 & 0.00 & 0.00 & 0.00 & 0.00 \\
\hline 0.00 & 0.00 & 0.00 & 0.00 & 0.00 & 0.00 & 0.00 & 0.00 \\
\hline 4.92 & 7.00 & 6.03 & 3.54 & 4.08 & 5.21 & 0.00 & 3.43 \\
\hline 10.67 & 7.75 & 8.44 & 10.00 & 9.04 & 10.84 & 6.95 & 4.90 \\
\hline 13.46 & 15.39 & 14.51 & 11.82 & 14.70 & 9.65 & 10.14 & 8.17 \\
\hline 17.69 & 17.84 & 18.33 & 18.80 & 15.45 & 17.59 & 14.63 & 13.94 \\
\hline 18.49 & 17.07 & 14.84 & 13.08 & 13.39 & 17.50 & 18.46 & 18.23 \\
\hline 10.56 & 10.37 & 13.26 & 8.44 & 8.58 & 10.08 & 11.07 & 16.50 \\
\hline 11.15 & 13.71 & 13.18 & 9.50 & 9.54 & 11.77 & 10.52 & 10.55 \\
\hline 9.59 & 9.04 & 10.28 & 8.86 & 9.69 & 6.91 & 11.44 & 13.72 \\
\hline 9.85 & 10.82 & 10.81 & 8.45 & 7.99 & 13.90 & 11.26 & 7.20 \\
\hline 6.14 & 7.35 & 7.50 & 10.32 & 10.39 & 7.26 & 9.96 & 9.19 \\
\hline 11.32 & 10.62 & 10.91 & 9.09 & 10.91 & 8.44 & 9.72 & 7.97 \\
\hline 8.43 & 10.20 & 11.93 & 12.02 & 9.02 & 10.44 & 12.23 & 7.94 \\
\hline 11.27 & 10.33 & 11.73 & 13.77 & 10.03 & 13.43 & 10.81 & 12.04 \\
\hline 13.38 & 14.01 & 17.17 & 16.83 & 12.95 & 15.60 & 14.24 & 12.44 \\
\hline 17.71 & 15.66 & 13.53 & 16.18 & 15.22 & 18.55 & 15.65 & 18.29 \\
\hline 14.52 & 9.91 & 12.12 & 13.12 & 12.32 & 15.06 & 13.17 & 15.99 \\
\hline 7.26 & 7.58 & 8.36 & 7.64 & 8.44 & 9.50 & 9.45 & 11.85 \\
\hline 3.39 & 3.78 & 2.80 & 4.54 & 5.04 & 4.50 & 6.37 & 4.70 \\
\hline 0.00 & 0.00 & 0.00 & 0.00 & 2.74 & 2.65 & 0.00 & 3.20 \\
\hline 0.00 & 0.00 & 0.00 & 0.00 & 0.00 & 0.00 & 0.00 & 0.00 \\
\hline 0.00 & 0.00 & 0.00 & 0.00 & 0.00 & 0.00 & 0.00 & 0.00 \\
\hline
\end{tabular}


Co-60 Input R matrix

\begin{tabular}{|c|c|c|c|c|c|c|c|}
\hline 0.00 & 0.00 & 0.00 & 0.00 & 0.00 & 0.00 & 0.00 & 0.00 \\
\hline 0.00 & 0.00 & 0.00 & 0.00 & 0.00 & 0.00 & 0.00 & 0.00 \\
\hline 0.00 & 2.63 & 3.16 & 0.00 & 0.00 & 0.00 & 0.00 & 0.00 \\
\hline 0.00 & 9.51 & 10.82 & 0.00 & 0.00 & 0.00 & 2.21 & 2.02 \\
\hline 4.74 & 0.00 & 2.55 & 7.84 & 0.00 & 0.00 & 0.00 & 1.88 \\
\hline 4.92 & 0.00 & 0.00 & 3.69 & 2.62 & 2.05 & 2.85 & 0.00 \\
\hline 3.35 & 2.70 & 3.45 & 2.50 & 5.93 & 4.41 & 3.83 & 4.60 \\
\hline 0.00 & 0.00 & 2.46 & 0.00 & 7.21 & 0.00 & 1.93 & 0.00 \\
\hline 2.74 & 0.00 & 3.29 & 2.05 & 0.00 & 3.23 & 2.79 & 3.84 \\
\hline 4.26 & 0.00 & 2.79 & 2.30 & 0.00 & 6.35 & 3.55 & 2.79 \\
\hline 0.00 & 2.48 & 2.44 & 0.00 & 3.87 & 5.43 & 0.00 & 0.00 \\
\hline 2.74 & 2.89 & 0.00 & 2.16 & 1.93 & 3.00 & 2.27 & 2.11 \\
\hline 2.10 & 2.02 & 0.00 & 2.76 & 2.42 & 0.00 & 5.96 & 0.00 \\
\hline 0.00 & 0.00 & 2.53 & 2.75 & 0.00 & 0.00 & 4.92 & 3.99 \\
\hline 3.15 & 0.00 & 0.00 & 3.23 & 2.49 & 2.95 & 2.43 & 2.75 \\
\hline 4.03 & 4.98 & 4.14 & 3.88 & 3.36 & 3.03 & 2.77 & 4.63 \\
\hline 2.82 & 2.51 & 2.40 & 3.64 & 3.42 & 4.23 & 4.17 & 8.03 \\
\hline 2.77 & 0.00 & 0.00 & 0.00 & 0.00 & 2.48 & 2.97 & 0.00 \\
\hline 0.00 & 0.00 & 0.00 & 2.00 & 2.13 & 0.00 & 2.66 & 0.00 \\
\hline 0.00 & 2.02 & 0.00 & 0.00 & 0.00 & 0.00 & 0.00 & 0.00 \\
\hline 0.00 & 0.00 & 0.00 & 0.00 & 0.00 & 0.00 & 0.00 & 0.00 \\
\hline 0.00 & 0.00 & 0.00 & 0.00 & 0.00 & 0.00 & 0.00 & 0.00 \\
\hline 0.00 & 0.00 & 0.00 & 0.00 & 0.00 & 0.00 & 0.00 & 0.00 \\
\hline
\end{tabular}


Ag-110m Input R matrix

\begin{tabular}{|c|c|c|c|c|c|c|c|}
\hline 0.00 & 0.00 & 0.00 & 0.00 & 0.00 & 0.00 & 0.00 & 0.00 \\
\hline 0.00 & 0.00 & 0.00 & 9.35 & 0.00 & 0.00 & 0.00 & 0.00 \\
\hline 0.00 & 10.83 & 12.59 & 12.13 & 10.23 & 0.00 & 0.00 & 0.00 \\
\hline 13.50 & 0.00 & 17.07 & 12.76 & 22.88 & 16.93 & 16.90 & 10.74 \\
\hline 15.58 & 15.51 & 16.86 & 14.74 & 21.31 & 22.48 & 21.40 & 12.57 \\
\hline 0.00 & 15.04 & 16.39 & 0.00 & 20.56 & 17.30 & 15.54 & 9.16 \\
\hline 10.87 & 9.00 & 12.72 & 8.95 & 16.31 & 9.47 & 15.99 & 14.35 \\
\hline 14.46 & 13.00 & 14.70 & 0.00 & 11.52 & 11.34 & 14.97 & 13.67 \\
\hline 13.21 & 10.40 & 14.18 & 9.53 & 8.55 & 0.00 & 10.55 & 0.00 \\
\hline 12.37 & 10.47 & 0.00 & 13.00 & 0.00 & 0.00 & 13.44 & 9.96 \\
\hline 0.00 & 13.83 & 9.09 & 10.13 & 0.00 & 10.05 & 0.00 & 0.00 \\
\hline 11.37 & 11.92 & 11.67 & 0.00 & 10.87 & 10.97 & 9.42 & 0.00 \\
\hline 10.08 & 13.70 & 10.32 & 11.74 & 17.66 & 0.00 & 0.00 & 10.90 \\
\hline 11.42 & 13.63 & 9.92 & 12.97 & 13.34 & 12.45 & 0.00 & 10.80 \\
\hline 11.86 & 12.52 & 10.06 & 9.90 & 14.04 & 11.97 & 9.10 & 9.32 \\
\hline 13.62 & 12.46 & 13.39 & 14.87 & 14.01 & 0.00 & 10.65 & 9.06 \\
\hline 19.70 & 20.29 & 20.92 & 18.19 & 11.98 & 0.00 & 15.97 & 12.69 \\
\hline 19.17 & 24.68 & 18.93 & 11.41 & 21.08 & 21.13 & 21.68 & 11.54 \\
\hline 19.88 & 18.47 & 17.38 & 14.12 & 22.59 & 22.03 & 18.87 & 19.69 \\
\hline 12.62 & 12.75 & 10.24 & 12.25 & 13.29 & 11.79 & 16.03 & 17.20 \\
\hline 0.00 & 0.00 & 0.00 & 0.00 & 0.00 & 0.00 & 0.00 & 0.00 \\
\hline 0.00 & 0.00 & 0.00 & 0.00 & 0.00 & 0.00 & 0.00 & 0.00 \\
\hline 0.00 & 0.00 & 0.00 & 0.00 & 0.00 & 0.00 & 0.00 & 0.00 \\
\hline
\end{tabular}




\section{Inner Ring 4 Axial Middle}

Angles for Middle IR-04 Scan

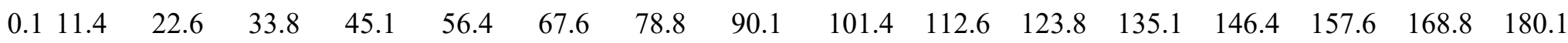

Cs-137 Input R matrix

\begin{tabular}{|c|c|c|c|c|c|c|c|c|c|c|c|c|c|c|c|c|}
\hline 2.35 & 0.00 & 0.00 & 1.44 & 0.00 & 0.00 & 0.00 & 0.00 & 0.00 & 0.00 & 0.00 & 0.00 & 0.00 & 0.00 & 0.00 & 1.51 & 1.48 \\
\hline 25.59 & 19.43 & 16.61 & 14.25 & 10.41 & 10.86 & 8.74 & 7.18 & 4.61 & 5.53 & 4.86 & 3.88 & & 12.08 & 15.69 & 27.39 & 32.91 \\
\hline 41.86 & 38.66 & 42.14 & 39.51 & 30.71 & 29.72 & 28.88 & 21.24 & 22.50 & 25.79 & 27.55 & 27.56 & 34.99 & 42.46 & 44.18 & 41.88 & 68.32 \\
\hline 90.03 & 80.94 & 78.54 & 4.59 & 60.93 & 66.00 & 55.60 & 51.40 & 47.38 & 49.26 & 53.56 & 66.25 & 82.10 & 92.73 & 77.67 & 104.35 & 116.02 \\
\hline 181.10 & 170.45 & 161.59 & 161.80 & 142.60 & 140.60 & 138.07 & 125.94 & 113.28 & 126.36 & 135.22 & 150.20 & 159.27 & 160.53 & 166.55 & 178.36 & 195.66 \\
\hline 234.16 & 73.92 & 5.92 & 2.02 & 266.54 & 254.83 & 236.90 & 245.34 & 240.80 & 259.15 & 245.76 & 245.55 & 265.38 & 279.51 & 280.88 & 275.71 & 282.04 \\
\hline 184.06 & 188.91 & 93.13 & 192.70 & 226.03 & 234.79 & 241.01 & 264.64 & 278.88 & 285.42 & 266.75 & 263.06 & 233.52 & 230.78 & 232.15 & 210.64 & 198.19 \\
\hline 172.77 & 157.69 & 170.13 & 54.86 & 173.93 & 174.88 & 195.45 & 89.12 & 187.54 & 193.97 & 201.25 & 192.07 & 177.83 & 168.34 & 175.72 & 183.53 & 186.48 \\
\hline 151.25 & 166.12 & 144.92 & 154.21 & 154.52 & 160.01 & 156.10 & 157.79 & 159.90 & 163.80 & 162.64 & 164.65 & 160.96 & 159.48 & 160.32 & 153.99 & 149.67 \\
\hline 139.44 & 139.97 & 48.83 & 43.34 & 148.30 & 147.77 & 147.56 & 154.10 & 155.47 & 139.97 & 147.03 & 149.04 & 144.71 & 150.83 & 159.58 & 137.75 & 140.07 \\
\hline 141.55 & 140.28 & 45.98 & 149.14 & 139.86 & 146.29 & 151.25 & 146.08 & 151.46 & 148.19 & 137.12 & 137.22 & 145.56 & 148.72 & 154.42 & 143.24 & 148.09 \\
\hline 153.57 & 149.14 & 145.98 & 157.79 & 151.04 & 148.72 & 155.15 & 145.98 & 136.38 & 140.28 & 135.11 & 140.28 & 142.50 & 163.38 & 149.99 & 149.88 & 141.65 \\
\hline 167.92 & 160.11 & 141.23 & 160.85 & 163.07 & 149.56 & 154.42 & 139.65 & 143.66 & 147.77 & 153.05 & 143.66 & 147.14 & 150.94 & 160.96 & 145.03 & 154.31 \\
\hline 183.21 & 168.23 & 185.64 & 173.93 & 171.61 & 170.55 & 153.78 & 152.83 & 147.98 & 153.89 & 164.65 & 163.07 & 162.01 & 152.41 & 147.56 & 167.07 & 179.63 \\
\hline 226.14 & 31.73 & 221.39 & 197.66 & 194.08 & 191.44 & 191.97 & 189.75 & 178.15 & 183.42 & 188.06 & 196.08 & 182.05 & 171.40 & 187.43 & 195.02 & 209.05 \\
\hline 278.35 & 271.60 & 281.30 & 271.07 & 260.42 & 264.74 & 252.72 & 247.34 & 260.74 & 254.41 & 256.10 & 242.81 & 251.35 & 260.10 & 256.52 & 246.81 & 251.35 \\
\hline 165.91 & 179.41 & 186.27 & 186.06 & 205.78 & 216.75 & 226.88 & 235.63 & 240.70 & 243.54 & 228.35 & 231.31 & 203.78 & 211.16 & 179.73 & 174.88 & 153.57 \\
\hline 97.48 & 106.64 & 113.70 & 130.37 & 117.61 & 119.08 & 126.99 & 115.28 & 134.27 & 127.73 & 122.77 & 115.92 & 101.23 & 100.35 & 101.09 & 75.64 & 75.40 \\
\hline 47.98 & 51.24 & 52.44 & 4.81 & 72.51 & 65.21 & 69.78 & 2.89 & 63.90 & 43.88 & 44.50 & 45.63 & 38.40 & 41.82 & 45.61 & 44.33 & 36.34 \\
\hline 15.03 & 13.90 & 17.04 & 27.36 & 33.09 & 38.36 & 37.58 & 31.23 & 26.25 & 23.18 & 23.72 & 21.83 & 18.82 & 18.11 & 18.47 & 13.37 & 12.86 \\
\hline 1.72 & 0.00 & 0.00 & 0.00 & 1.42 & 2.06 & 5.43 & 6.25 & 5.21 & 5.18 & 5.81 & 3.06 & 1.88 & 2.95 & 2.25 & 0.00 & 0.00 \\
\hline
\end{tabular}




\section{Cs-134 Input R matrix}

\begin{tabular}{|c|c|c|c|c|c|c|c|c|c|c|c|c|c|c|c|c|}
\hline 3.11 & 0.00 & 0.00 & 0.00 & 0.00 & 0.00 & 0.00 & 0.00 & 0.00 & 0.00 & 0.00 & 0.00 & 0.00 & 0.00 & 0.00 & 0.00 & 0.00 \\
\hline 22.39 & 18.66 & 17.94 & 15.88 & 7.76 & .98 & 42 & 5.44 & & & & & 7.51 & 13.22 & & & \\
\hline 47.00 & 49.52 & 41.48 & 40.54 & 28.67 & 30.87 & 26.39 & 21.85 & 23.85 & 26.24 & 21.00 & 27.93 & 33.39 & 43.00 & 44.39 & 50.30 & 63.82 \\
\hline 92.00 & 89.58 & 82.30 & 83.43 & 60.43 & 66.54 & 56.65 & 55.82 & 48.39 & 51.52 & 49.04 & 68.26 & 88.50 & 92.78 & 91.58 & 102.99 & 122.52 \\
\hline 184.01 & 164.12 & 160.36 & 162.64 & 151.19 & 141.91 & 129.88 & 118.78 & 122.52 & 132.80 & 140.91 & 161.49 & 172.93 & 160.38 & 164.64 & 183.86 & 202.79 \\
\hline 231.94 & 59.33 & 251.94 & 253.68 & 258.90 & 257.38 & 237.60 & 239.55 & 222.60 & 246.07 & 222.81 & 241.94 & 262.59 & 273.03 & 270.42 & 264.55 & 66.29 \\
\hline 184.27 & 84.36 & 193.25 & 79.58 & 208.90 & 219.77 & 236.07 & 256.51 & 266.94 & 263.46 & 259.77 & 247.16 & 220.21 & 206.25 & 206.51 & 211.68 & 203.14 \\
\hline 163.40 & 3.93 & 3.06 & 17.45 & 9.12 & 163.14 & 175.84 & 173.34 & 181.03 & 181.40 & 186.97 & 180.82 & 175.23 & 161.23 & 169.58 & 188.51 & 72.45 \\
\hline 153.95 & 55.27 & 46.73 & 141.04 & 138.54 & 50.75 & 153.47 & 152.19 & 153.56 & 160.19 & 161.34 & 151.91 & 145.47 & 147.71 & 163.73 & 156.80 & 147.23 \\
\hline 140.86 & 28.82 & 153.36 & 140.58 & 44.41 & 142.78 & 145.91 & 155.62 & 158.34 & 144.38 & 138.82 & 146.64 & 138.43 & 157.56 & 141.21 & 141.86 & 142.12 \\
\hline 140.12 & 7.86 & 50.62 & 139.19 & 139.19 & 142.64 & 144.58 & 144.60 & 133.97 & 141.12 & 137.73 & 131.78 & 145.30 & 139.64 & 145.06 & 151.10 & 132.45 \\
\hline 143.12 & 13.82 & 144.06 & 149.91 & 140.84 & 140.60 & 142.60 & 141.30 & 138.17 & 137.84 & 129.12 & 137.34 & 133.25 & 147.58 & 146.04 & 141.14 & 138.64 \\
\hline 158.08 & 52.99 & 146.25 & 54.54 & 154.95 & 142.80 & 145.10 & 145.56 & 141.75 & 130.17 & 146.54 & 132.75 & 146.95 & 135.32 & 141.49 & 137.30 & 157.43 \\
\hline 184.77 & 161.84 & 176.14 & 159.32 & 158.51 & 160.12 & 144.25 & 151.12 & 146.69 & 145.91 & 147.49 & 148.45 & 152.32 & 147.45 & 144.99 & 165.08 & 168.43 \\
\hline 219.55 & 20.64 & 207.32 & 201.88 & 176.86 & 170.19 & 185.75 & 172.03 & 169.86 & 178.29 & 169.51 & 170.97 & 168.51 & 168.17 & 176.56 & 188.51 & 186.32 \\
\hline 264.12 & 255.42 & 263.46 & 259.99 & 242.60 & 257.81 & 239.77 & 246.07 & 241.94 & 241.29 & 240.86 & 238.03 & 240.64 & 241.94 & 254.99 & 243.47 & 226.73 \\
\hline 170.43 & 192.71 & 182.73 & 198.27 & 202.95 & 215.99 & 219.99 & 221.08 & 223.68 & 224.99 & 239.12 & 220.64 & 198.29 & 199.88 & 182.03 & 170.60 & 132.32 \\
\hline 92.86 & 97.67 & 115.10 & 123.23 & 120.82 & 126.93 & 116.52 & 120.32 & 129.71 & 131.30 & 122.32 & 117.47 & 101.67 & 100.65 & 99.39 & 82.93 & 75.43 \\
\hline 50.56 & 48.26 & 4.82 & 69.67 & 9.58 & 6.34 & 72.50 & 66.28 & 1.02 & 49.17 & 49.19 & 49.5 & 40.56 & 49.45 & 44.89 & 1.50 & 34.32 \\
\hline 14.26 & 17.54 & 16.55 & 26.02 & 35.69 & 40.45 & 35.87 & 31.63 & 27.67 & 25.22 & 18.29 & 24.87 & 23.82 & 21.09 & 22.22 & 16.24 & 11.12 \\
\hline 0.00 & 00 & 0.00 & 0.00 & .01 & 3.17 & 6.00 & 3.71 & 5.57 & 3.94 & 4.10 & 0.00 & 0.00 & 0.00 & 0.00 & 0.00 & 0.00 \\
\hline
\end{tabular}




\section{Inner Ring 5 Axial Middle}

Angles for Middle IR-05 Scan

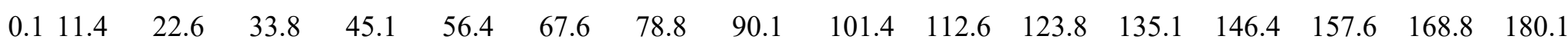

\section{Cs-137 Input R matrix}

\begin{tabular}{|c|c|c|c|c|c|c|c|c|c|c|c|c|c|c|c|c|}
\hline 2.06 & 0.00 & 0.00 & 0.00 & 1.24 & 0.00 & 0.00 & 0.00 & 0.00 & 0.00 & 0.00 & 0.00 & 0.00 & 0.00 & 0.00 & 1.63 & 0.00 \\
\hline 0.00 & 0.00 & 0.00 & 1.04 & 1.21 & 0.00 & 1.15 & 0.00 & 0.00 & 0.00 & 0.00 & 0.00 & 1.49 & 0.00 & 0.00 & 0.00 & 0.00 \\
\hline 11.43 & 5.42 & 7.17 & 4.69 & 4.67 & 1.85 & 0.00 & 0.00 & 0.00 & 0.00 & 0.00 & 0.00 & 0.00 & 0.00 & 0.00 & 0.00 & 0.00 \\
\hline 31.05 & 30.13 & 26.69 & 5.15 & 20.18 & 18.65 & 10.97 & 7.14 & 4.48 & 2.22 & 1.00 & 0.00 & 0.00 & 0.00 & 0.00 & 0.00 & 0.00 \\
\hline 87.90 & 75.52 & 74.58 & 72.18 & 63.78 & 54.77 & 36.80 & 28.07 & 21.98 & 17.79 & 14.51 & 13.21 & 9.35 & 7.93 & 6.17 & 9.94 & 10.30 \\
\hline 177.94 & 174.14 & 163.28 & 142.08 & 133.11 & 106.21 & 85.40 & 79.15 & 58.25 & 53.44 & 44.75 & 38.94 & 38.35 & 37.84 & 29.36 & 34.40 & 33.76 \\
\hline 289.43 & 269.81 & 267.38 & 272.76 & 243.65 & 217.70 & 185.53 & 160.53 & 136.06 & 112.12 & 101.57 & 90.99 & 87.02 & 92.12 & 80.57 & 83.21 & 83.19 \\
\hline 213.69 & 08.31 & 08.95 & 23.08 & 243.33 & 264.74 & 277.08 & 264.11 & 247.66 & 218.76 & 192.28 & 177.73 & 175.30 & 159.90 & 162.54 & 156.00 & 165.18 \\
\hline 183.32 & 172.35 & 71.50 & 175.20 & 169.50 & 172.24 & 197.45 & 212.64 & 245.02 & 267.28 & 274.34 & 266.01 & 258.63 & 264.11 & 252.40 & 262.63 & 256.31 \\
\hline 165.91 & 170.87 & 156.32 & 152.10 & 149.35 & 154.31 & 176.36 & 175.83 & 189.01 & 179.73 & 185.22 & 192.28 & 195.66 & 197.03 & 212.43 & 196.82 & 214.12 \\
\hline 144.71 & 151.36 & 151.36 & 145.56 & 153.26 & 151.25 & 151.15 & 159.48 & 164.54 & 162.75 & 156.21 & 165.18 & 161.17 & 170.87 & 174.03 & 153.36 & 167.50 \\
\hline 132.48 & 136.38 & 153.57 & 155.89 & 147.77 & 139.76 & 154.31 & 149.35 & 149.99 & 148.62 & 155.15 & 146.93 & 160.53 & 162.22 & 160.96 & 141.65 & 146.93 \\
\hline 137.54 & 128.26 & 155.37 & 167.07 & 153.68 & 142.39 & 144.40 & 153.99 & 133.74 & 134.59 & 152.73 & 142.18 & 147.46 & 151.89 & 154.52 & 148.83 & 133.00 \\
\hline 154.84 & 141.23 & 147.35 & 163.28 & 170.24 & 153.89 & 149.04 & 141.44 & 142.50 & 145.77 & 145.56 & 149.67 & 142.08 & 153.05 & 148.93 & 140.18 & 126.99 \\
\hline 161.59 & 178.04 & 163.17 & 159.06 & 167.92 & 170.34 & 143.55 & 150.30 & 154.42 & 144.82 & 148.51 & 156.42 & 163.59 & 151.25 & 140.81 & 122.67 & 138.28 \\
\hline 203.78 & 204.52 & 208.95 & 187.22 & 185.64 & 186.27 & 176.67 & 168.44 & 148.40 & 173.09 & 163.91 & 167.28 & 160.11 & 154.21 & 134.59 & 141.65 & \\
\hline 270.44 & 280.04 & 276.66 & 286.89 & 259.89 & 241.86 & 215.59 & 205.68 & 185.95 & 177.20 & 187.85 & 171.93 & 172.87 & 161.69 & 164.96 & 176.36 & 176.88 \\
\hline 175.51 & 164.54 & 179.20 & 182.68 & 213.38 & 239.96 & 279.09 & 287.00 & 275.82 & 258.94 & 231.84 & 214.54 & 182.68 & 189.43 & 208.00 & 203.88 & \\
\hline 90.07 & 97.58 & 88.38 & 86.29 & 105.90 & 134.90 & 155.58 & 180.15 & 217.17 & 237.32 & 261.05 & 270.86 & 291.22 & 289.85 & 292.91 & 282.57 & 280.99 \\
\hline 39.09 & 42.97 & 56.90 & 56.93 & 58.97 & 64.96 & 82.22 & 92.88 & 115.92 & 134.69 & 147.14 & 168.34 & 186.59 & 196.92 & 187.11 & 185.95 & 181.84 \\
\hline 12.32 & 17.53 & 19.45 & 23.66 & 32.00 & 32.01 & 33.01 & 37.80 & 47.13 & 66.83 & 74.31 & 87.78 & 87.26 & 92.92 & 82.24 & 85.28 & 86.14 \\
\hline 0.00 & 0.00 & 1.04 & 1.52 & 3.08 & 4.36 & 8.81 & 12.55 & 14.88 & 24.69 & 27.51 & 39.69 & 37.74 & 34.86 & 33.24 & 30.22 & 32.32 \\
\hline 0.00 & 0.00 & 0.00 & 0.00 & 0.00 & 0.00 & 0.00 & 0.00 & 2.24 & 4.50 & 6.23 & 8.61 & 9.61 & 10.94 & 11.17 & 9.39 & 11.93 \\
\hline
\end{tabular}




\section{Cs-134 Input R matrix}

\begin{tabular}{|c|c|c|c|c|c|c|c|c|c|c|c|c|c|c|c|c|}
\hline 0.00 & 0.00 & 0.00 & 0.00 & 0.00 & 0.00 & 0.00 & 0.00 & 0.00 & 0.00 & 0.00 & 0.00 & 0.00 & 0.00 & 0.00 & 0.00 & 0.00 \\
\hline 0.00 & 0.00 & 0.00 & 0.00 & 0.00 & 0.00 & 0.00 & 0.00 & 0.00 & 0.00 & 0.00 & 0.00 & 0.00 & 0.00 & 0.00 & 0.00 & 0.00 \\
\hline 13.48 & 6.84 & 7.22 & 6.27 & 3.69 & 0.00 & 0.00 & 0.00 & 0.00 & 0.00 & 0.00 & 0.00 & 0.00 & 0.00 & 0.00 & 0.00 & 0.00 \\
\hline 33.30 & 33.61 & 31.11 & 28.26 & 23.09 & 19.10 & 11.98 & 9.93 & 4.50 & 2.90 & 0.00 & 0.00 & 0.00 & 0.00 & 0.00 & 0.00 & 0.00 \\
\hline 99.80 & 85.69 & 83.67 & 92.19 & 74.56 & 62.58 & 45.35 & 35.72 & 26.02 & 22.82 & 17.01 & 14.53 & 9.85 & 9.45 & 8.44 & 9.05 & 11.50 \\
\hline 202.90 & 197.79 & 182.40 & 169.62 & 145.75 & 132.54 & 97.60 & 83.89 & 72.97 & 58.32 & 52.04 & 42.82 & 41.78 & 41.28 & 39.54 & 38.91 & 45.80 \\
\hline 326.29 & 309.77 & 295.42 & 294.98 & 279.12 & 242.81 & 195.75 & 178.71 & 154.64 & 133.95 & 115.32 & 98.39 & 110.04 & 102.97 & 92.69 & 97.30 & 95.41 \\
\hline 235.21 & 217.60 & 232.38 & 249.77 & 264.33 & 276.29 & 294.98 & 283.90 & 264.99 & 243.47 & 216.05 & 197.66 & 188.86 & 182.10 & 178.86 & 173.82 & 177.86 \\
\hline 204.45 & 191.64 & 181.51 & 190.71 & 185.77 & 183.10 & 212.29 & 225.64 & 251.94 & 280.64 & 295.85 & 287.81 & 283.46 & 281.51 & 266.73 & 276.29 & 278.46 \\
\hline 174.21 & 180.73 & 169.77 & 162.25 & 156.84 & 178.60 & 181.62 & 188.12 & 205.45 & 194.38 & 207.66 & 208.14 & 213.75 & 207.01 & 232.81 & 208.47 & 209.60 \\
\hline 164.23 & 171.47 & 163.95 & 155.97 & 156.30 & 163.25 & 161.58 & 174.66 & 177.53 & 181.93 & 162.75 & 179.73 & 176.95 & 178.71 & 186.66 & 170.71 & 173.51 \\
\hline 143.86 & 152.88 & 163.82 & 160.19 & 158.40 & 156.27 & 159.45 & 168.45 & 163.60 & 154.93 & 160.69 & 152.23 & 156.60 & 163.97 & 172.27 & 165.47 & 155.73 \\
\hline 147.91 & 145.17 & 165.64 & 173.69 & 168.71 & 162.14 & 154.69 & 167.49 & 155.08 & 156.75 & 164.73 & 155.88 & 157.12 & 162.53 & 164.38 & 155.77 & 142.04 \\
\hline 166.84 & 158.77 & 165.19 & 170.10 & 182.75 & 167.21 & 153.60 & 148.10 & 158.64 & 148.01 & 156.06 & 169.23 & 158.88 & 165.88 & 160.86 & 154.04 & 140.06 \\
\hline 181.23 & 187.56 & 178.64 & 172.69 & 194.51 & 181.86 & 165.38 & 155.51 & 164.69 & 163.25 & 158.01 & 167.51 & 178.29 & 157.86 & 160.71 & 137.19 & 156.90 \\
\hline 224.34 & 221.94 & 219.12 & 206.79 & 199.82 & 204.45 & 197.62 & 173.64 & 170.40 & 178.03 & 174.32 & 171.27 & 176.14 & 175.64 & 149.08 & 165.56 & 169.80 \\
\hline 303.46 & 295.20 & 285.20 & 296.94 & 270.86 & 270.20 & 241.94 & 209.47 & 199.71 & 197.55 & 209.36 & 185.77 & 174.38 & 178.58 & 179.43 & 182.53 & 195.69 \\
\hline 190.86 & 188.14 & 188.66 & 210.66 & 240.20 & 258.46 & 310.42 & 306.51 & 290.42 & 278.03 & 245.86 & 225.64 & 207.66 & 224.55 & 218.03 & 218.25 & 217.60 \\
\hline 101.71 & 108.10 & 99.32 & 98.23 & 118.78 & 140.86 & 179.23 & 214.40 & 244.55 & 271.94 & 280.42 & 296.51 & 302.59 & 322.16 & 313.46 & 312.59 & 309.33 \\
\hline 51.50 & 54.71 & 63.82 & 72.15 & 69.84 & 75.80 & 95.86 & 109.17 & 137.25 & 140.36 & 169.36 & 188.32 & 208.27 & 225.42 & 205.27 & 214.90 & 203.71 \\
\hline 14.74 & 16.88 & 20.89 & 26.17 & 34.76 & 42.11 & 47.58 & 47.35 & 52.02 & 76.24 & 83.63 & 98.84 & 109.78 & 108.99 & 96.10 & 93.00 & 99.21 \\
\hline 0.00 & 0.00 & 0.00 & 2.95 & 0.00 & 7.00 & 7.43 & 11.20 & 16.85 & 22.02 & 35.32 & 38.39 & 40.26 & 42.82 & 41.74 & 32.50 & 36.78 \\
\hline 0.00 & 0.00 & 0.00 & 0.00 & 0.00 & 0.00 & 0.00 & 0.00 & 0.00 & 2.91 & 6.84 & 11.04 & 15.32 & 11.26 & 8.19 & 13.49 & 9.73 \\
\hline
\end{tabular}




\section{Inner Ring 7 Axial Middle}

Angles for Middle IR-07 Scan

$0,22.5,45,67.5,90,112.5,135,157.5$

\section{Cs-137 Input R matrix}

\begin{tabular}{|c|c|c|c|c|c|c|c|}
\hline 0.00 & 0.00 & 0.00 & 0.00 & 0.00 & 0.00 & 0.00 & 0.00 \\
\hline 0.00 & 0.00 & 0.00 & 0.00 & 0.00 & 0.00 & 2.11 & 0.00 \\
\hline 1.33 & 1.95 & 4.96 & 5.78 & 8.48 & 7.24 & 6.91 & 5.41 \\
\hline 7.65 & 7.74 & 9.02 & 11.35 & 10.90 & 13.36 & 13.39 & 9.27 \\
\hline 12.06 & 9.96 & 11.86 & 12.48 & 15.76 & 14.96 & 12.13 & 18.00 \\
\hline 11.53 & 16.34 & 16.17 & 19.20 & 15.58 & 14.79 & 19.06 & 18.09 \\
\hline 16.46 & 16.62 & 14.65 & 16.39 & 15.06 & 13.81 & 13.44 & 21.40 \\
\hline 13.48 & 13.53 & 13.45 & 12.54 & 13.02 & 13.26 & 10.59 & 13.91 \\
\hline 13.33 & 9.00 & 7.42 & 12.25 & 12.35 & 10.57 & 13.43 & 13.34 \\
\hline 9.50 & 11.83 & 14.53 & 12.42 & 11.11 & 9.68 & 9.47 & 7.97 \\
\hline 9.40 & 10.00 & 10.11 & 12.63 & 10.29 & 14.07 & 10.90 & 10.53 \\
\hline 9.27 & 6.34 & 9.78 & 11.80 & 10.89 & 11.05 & 10.11 & 11.33 \\
\hline 9.77 & 10.11 & 6.49 & 9.14 & 11.41 & 10.87 & 10.19 & 9.51 \\
\hline 10.31 & 10.25 & 11.49 & 9.43 & 11.35 & 10.82 & 11.80 & 13.88 \\
\hline 9.07 & 12.80 & 14.21 & 12.79 & 13.35 & 11.07 & 13.25 & 12.69 \\
\hline 15.17 & 13.31 & 14.78 & 15.40 & 14.59 & 16.46 & 15.79 & 13.19 \\
\hline 18.16 & 18.82 & 18.85 & 15.72 & 14.61 & 14.74 & 12.89 & 18.00 \\
\hline 21.06 & 19.65 & 15.66 & 15.66 & 13.46 & 10.93 & 8.45 & 10.09 \\
\hline 14.03 & 17.52 & 11.67 & 12.31 & 8.74 & 9.57 & 9.08 & 8.60 \\
\hline 11.97 & 7.45 & 7.14 & 6.77 & 4.53 & 4.44 & 3.70 & 6.51 \\
\hline 5.09 & 2.77 & 2.34 & 1.61 & 0.00 & 0.00 & 0.00 & 0.00 \\
\hline 0.00 & 0.00 & 0.00 & 1.15 & 0.00 & 0.00 & 0.00 & 0.00 \\
\hline 0.00 & 0.00 & 0.00 & 0.00 & 0.00 & 0.00 & 0.00 & 1.95 \\
\hline
\end{tabular}




\section{Cs-134 Input R matrix}

\begin{tabular}{|c|c|c|c|c|c|c|c|}
\hline 0.00 & 0.00 & 0.00 & 0.00 & 0.00 & 0.00 & 0.00 & 0.00 \\
\hline 0.00 & 0.00 & 0.00 & 0.00 & 0.00 & 0.00 & 3.03 & 0.00 \\
\hline 0.00 & 3.42 & 5.79 & 6.93 & 7.28 & 8.24 & 9.27 & 8.66 \\
\hline 6.20 & 7.87 & 10.24 & 12.30 & 12.41 & 12.27 & 14.28 & 11.43 \\
\hline 14.02 & 13.21 & 14.80 & 14.49 & 17.42 & 17.32 & 16.43 & 15.16 \\
\hline 17.39 & 16.20 & 18.01 & 18.42 & 19.76 & 19.38 & 20.92 & 20.31 \\
\hline 16.39 & 17.85 & 17.09 & 17.78 & 13.26 & 16.61 & 18.21 & 19.56 \\
\hline 15.14 & 14.38 & 16.18 & 15.49 & 12.82 & 13.59 & 15.12 & 16.91 \\
\hline 13.87 & 8.89 & 12.36 & 10.63 & 10.97 & 12.12 & 13.22 & 14.82 \\
\hline 10.50 & 9.92 & 12.05 & 11.64 & 12.24 & 11.25 & 10.72 & 9.38 \\
\hline 11.47 & 8.62 & 12.76 & 13.06 & 8.00 & 13.13 & 11.69 & 9.84 \\
\hline 15.39 & 13.91 & 10.46 & 11.30 & 11.91 & 11.68 & 10.29 & 11.58 \\
\hline 12.13 & 10.39 & 11.84 & 12.25 & 9.51 & 10.64 & 13.64 & 11.92 \\
\hline 10.64 & 12.69 & 13.69 & 13.67 & 14.72 & 11.56 & 13.58 & 12.87 \\
\hline 11.99 & 14.24 & 13.99 & 12.58 & 15.27 & 14.37 & 10.12 & 11.89 \\
\hline 13.83 & 18.52 & 17.53 & 15.74 & 18.49 & 16.99 & 16.27 & 16.28 \\
\hline 21.94 & 20.27 & 19.53 & 20.68 & 21.33 & 16.91 & 15.58 & 16.71 \\
\hline 19.41 & 22.23 & 19.78 & 14.21 & 19.13 & 14.34 & 16.74 & 13.59 \\
\hline 17.80 & 13.76 & 14.36 & 9.19 & 10.57 & 6.50 & 10.27 & 12.09 \\
\hline 12.59 & 9.82 & 8.65 & 7.43 & 4.05 & 4.16 & 5.29 & 4.90 \\
\hline 7.36 & 4.20 & 2.43 & 0.00 & 0.00 & 0.00 & 0.00 & 0.00 \\
\hline 0.00 & 0.00 & 0.00 & 0.00 & 0.00 & 0.00 & 0.00 & 0.00 \\
\hline 0.00 & 0.00 & 0.00 & 0.00 & 0.00 & 0.00 & 0.00 & 0.00 \\
\hline
\end{tabular}


Eu-154 Input R matrix

\begin{tabular}{|c|c|c|c|c|c|c|c|}
\hline 0.00 & 0.00 & 0.00 & 0.00 & 0.00 & 0.00 & 0.00 & 0.00 \\
\hline 0.00 & 0.00 & 0.00 & 0.00 & 0.00 & 0.00 & 0.00 & 0.00 \\
\hline 0.00 & 0.00 & 0.00 & 0.00 & 0.00 & 0.00 & 0.00 & 0.00 \\
\hline 0.00 & 0.00 & 0.00 & 0.00 & 0.00 & 0.00 & 0.00 & 0.00 \\
\hline 0.00 & 0.00 & 0.00 & 0.00 & 0.00 & 0.00 & 0.00 & 0.00 \\
\hline 0.00 & 0.00 & 0.00 & 25.40 & 31.53 & 42.52 & 39.28 & 23.75 \\
\hline 24.28 & 70.11 & 87.46 & 79.31 & 73.93 & 58.36 & 69.42 & 76.74 \\
\hline 70.23 & 51.64 & 40.27 & 44.34 & 39.40 & 40.87 & 41.21 & 36.98 \\
\hline 37.54 & 32.65 & 32.41 & 33.04 & 35.17 & 47.48 & 35.76 & 34.23 \\
\hline 35.97 & 30.63 & 32.88 & 28.18 & 35.57 & 34.02 & 32.27 & 38.54 \\
\hline 33.67 & 26.84 & 28.00 & 31.05 & 25.15 & 32.67 & 32.21 & 34.34 \\
\hline 31.82 & 28.74 & 29.40 & 32.71 & 27.46 & 29.88 & 33.36 & 32.80 \\
\hline 36.01 & 30.75 & 28.01 & 23.82 & 29.60 & 28.40 & 38.56 & 40.66 \\
\hline 37.96 & 32.84 & 38.72 & 34.35 & 33.19 & 41.06 & 34.55 & 34.94 \\
\hline 40.77 & 47.70 & 35.15 & 46.19 & 42.57 & 47.10 & 47.77 & 47.05 \\
\hline 34.24 & 58.14 & 68.20 & 79.67 & 97.71 & 89.83 & 81.54 & 74.12 \\
\hline 92.63 & 77.98 & 62.90 & 30.03 & 11.75 & 0.00 & 4.81 & 23.56 \\
\hline 14.74 & 0.00 & 0.00 & 0.00 & 0.00 & 0.00 & 0.00 & 0.00 \\
\hline 0.00 & 0.00 & 0.00 & 0.00 & 0.00 & 0.00 & 0.00 & 0.00 \\
\hline 0.00 & 0.00 & 0.00 & 0.00 & 0.00 & 0.00 & 0.00 & 0.00 \\
\hline 0.00 & 0.00 & 0.00 & 0.00 & 0.00 & 0.00 & 0.00 & 0.00 \\
\hline 0.00 & 0.00 & 0.00 & 0.00 & 0.00 & 0.00 & 0.00 & 0.00 \\
\hline 0.00 & 0.00 & 0.00 & 0.00 & 0.00 & 0.00 & 0.00 & 0.00 \\
\hline
\end{tabular}


Co-60 Input R matrix

\begin{tabular}{|c|c|c|c|c|c|c|c|}
\hline 0.00 & 0.00 & 0.00 & 0.00 & 0.00 & 0.00 & 0.00 & 0.00 \\
\hline 0.00 & 0.00 & 0.00 & 0.00 & 0.00 & 0.00 & 0.00 & 0.00 \\
\hline 0.00 & 0.00 & 0.00 & 0.00 & 0.00 & 0.00 & 0.00 & 0.00 \\
\hline 0.00 & 0.00 & 0.00 & 0.00 & 0.00 & 2.34 & 0.00 & 2.30 \\
\hline 2.46 & 0.00 & 0.00 & 0.00 & 0.00 & 2.36 & 0.00 & 0.00 \\
\hline 0.00 & 0.00 & 0.00 & 0.00 & 0.00 & 0.00 & 0.00 & 3.39 \\
\hline 10.60 & 0.00 & 0.00 & 0.00 & 0.00 & 0.00 & 0.00 & 1.98 \\
\hline 0.00 & 0.00 & 0.00 & 0.00 & 0.00 & 0.00 & 0.00 & 0.00 \\
\hline 0.00 & 10.63 & 0.00 & 0.00 & 0.00 & 0.00 & 2.05 & 0.00 \\
\hline 0.00 & 0.00 & 0.00 & 0.00 & 0.00 & 0.00 & 0.00 & 0.00 \\
\hline 0.00 & 0.00 & 8.21 & 0.00 & 0.00 & 0.00 & 0.00 & 0.00 \\
\hline 0.00 & 0.00 & 0.00 & 0.00 & 0.00 & 0.00 & 0.00 & 0.00 \\
\hline 0.00 & 0.00 & 0.00 & 0.00 & 0.00 & 0.00 & 0.00 & 0.00 \\
\hline 0.00 & 0.00 & 0.00 & 10.31 & 0.00 & 0.00 & 0.00 & 0.00 \\
\hline 0.00 & 0.00 & 0.00 & 0.00 & 0.00 & 0.00 & 0.00 & 0.00 \\
\hline 0.00 & 0.00 & 0.00 & 0.00 & 8.28 & 0.00 & 2.69 & 0.00 \\
\hline 0.00 & 0.00 & 0.00 & 0.00 & 0.00 & 2.46 & 0.00 & 0.00 \\
\hline 2.32 & 0.00 & 0.00 & 0.00 & 0.00 & 8.43 & 7.06 & 5.27 \\
\hline 0.00 & 1.88 & 0.00 & 0.00 & 2.18 & 0.00 & 5.02 & 7.62 \\
\hline 0.00 & 0.00 & 0.00 & 0.00 & 0.00 & 0.00 & 0.00 & 2.45 \\
\hline 0.00 & 0.00 & 0.00 & 0.00 & 0.00 & 0.00 & 0.00 & 0.00 \\
\hline 0.00 & 0.00 & 0.00 & 0.00 & 0.00 & 0.00 & 0.00 & 0.00 \\
\hline 0.00 & 0.00 & 0.00 & 0.00 & 0.00 & 0.00 & 0.00 & 0.00 \\
\hline
\end{tabular}




\section{Inner Ring 8 Axial Middle}

Angles for Middle IR-08 Scan

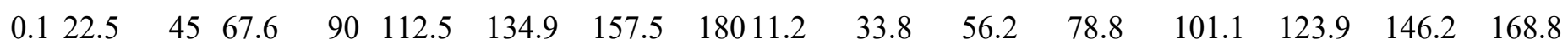

Cs-137 Input R matrix

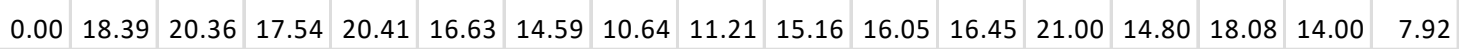
\begin{tabular}{|l|l|l|l|l|l|l|l|l|l|l|l|l|l|l|l|l|}
2.03 & 19.63 & 25.77 & 21.86 & 18.05 & 17.61 & 26.42 & 14.42 & 15.49 & 22.82 & 21.84 & 22.40 & 23.73 & 21.37 & 19.19 & 17.05 & 15.10 \\
\hline
\end{tabular} \begin{tabular}{|l|l|l|l|l|l|l|l|l|l|l|l|l|l|l|l|l|}
13.51 & 26.04 & 24.56 & 24.64 & 22.63 & 25.01 & 21.44 & 17.11 & 18.59 & 23.55 & 25.12 & 26.23 & 24.51 & 24.23 & 20.91 & 24.42 & 19.61 \\
\hline
\end{tabular} \begin{tabular}{|l|l|l|l|l|l|l|l|l|l|l|l|l|l|l|l|l|}
19.39 & 23.13 & 22.90 & 23.74 & 18.86 & 18.82 & 20.61 & 21.72 & 20.92 & 21.09 & 23.87 & 20.80 & 24.69 & 24.70 & 23.11 & 24.56 & 19.73 \\
\hline
\end{tabular} \begin{tabular}{|l|l|l|l|l|l|l|l|l|l|l|l|l|l|l|l|l|}
\hline 20.06 & 21.44 & 18.19 & 15.37 & 22.70 & 15.46 & 16.00 & 20.54 & 15.39 & 20.80 & 19.42 & 19.34 & 18.24 & 21.84 & 18.04 & 19.15 & 20.48 \\
\hline
\end{tabular} \begin{tabular}{|l|l|l|l|l|l|l|l|l|l|l|l|l|l|l|l|l|}
\hline 23.76 & 17.65 & 12.70 & 15.86 & 21.45 & 16.23 & 15.60 & 15.67 & 16.69 & 19.83 & 18.13 & 13.33 & 16.63 & 20.92 & 16.20 & 14.92 & 14.48 \\
\hline
\end{tabular} \begin{tabular}{|l|l|l|l|l|l|l|l|l|l|l|l|l|l|l|l|l|l|l|}
\hline 22.66 & 15.17 & 15.06 & 16.25 & 18.96 & 14.21 & 13.50 & 15.11 & 16.08 & 15.62 & 13.10 & 14.49 & 15.21 & 15.95 & 16.48 & 15.64 & 12.58 \\
\hline
\end{tabular} \begin{tabular}{|l|l|l|l|l|l|l|l|l|l|l|l|l|l|l|l|l|}
\hline 16.46 & 14.38 & 14.89 & 15.13 & 13.32 & 12.87 & 11.62 & 13.07 & 13.52 & 13.86 & 13.07 & 15.27 & 15.60 & 12.99 & 18.40 & 15.58 & 11.51 \\
\hline
\end{tabular}

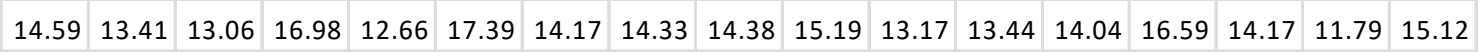
\begin{tabular}{|l|l|l|l|l|l|l|l|l|l|l|l|l|l|l|l|l|}
13.96 & 15.38 & 13.26 & 13.39 & 11.71 & 13.19 & 14.58 & 15.41 & 18.38 & 16.58 & 11.78 & 15.68 & 12.62 & 13.06 & 12.48 & 15.41 & 14.37 \\
\hline
\end{tabular}

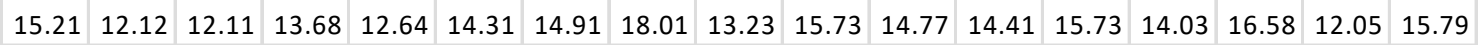

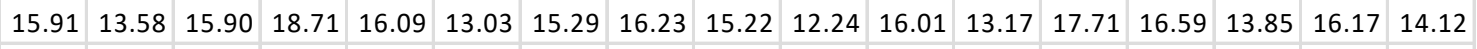
\begin{tabular}{|l|l|l|l|l|l|l|l|l|l|l|l|l|l|l|l|l|}
15.87 & 17.79 & 15.80 & 21.88 & 16.20 & 19.21 & 20.22 & 15.74 & 15.75 & 13.10 & 15.95 & 18.16 & 15.79 & 17.06 & 16.79 & 16.82 & 18.15 \\
\hline
\end{tabular}

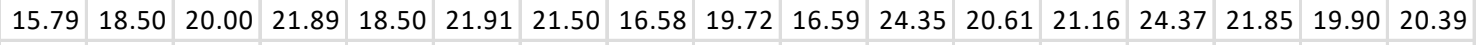
\begin{tabular}{|l|l|l|l|l|l|l|l|l|l|l|l|l|l|l|l|l|}
\hline 13.78 & 20.31 & 22.61 & 14.27 & 19.46 & 22.43 & 20.48 & 23.04 & 27.07 & 17.82 & 18.58 & 17.04 & 20.28 & 16.85 & 22.51 & 22.19 & 25.23 \\
\hline
\end{tabular} \begin{tabular}{|l|l|l|l|l|l|l|l|l|l|l|l|l|l|l|l|l|}
17.95 & 15.87 & 17.55 & 14.39 & 16.00 & 16.75 & 19.69 & 22.71 & 22.61 & 19.24 & 18.78 & 14.85 & 17.44 & 18.79 & 15.08 & 17.77 & 24.64 \\
\hline
\end{tabular}

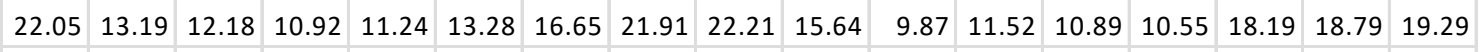

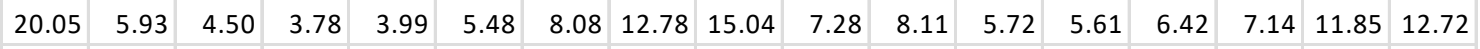
\begin{tabular}{|l|l|l|l|l|l|l|l|l|l|l|l|l|l|l|l|l|}
\hline 17.19 & 0.00 & 0.00 & 0.00 & 0.00 & 0.00 & 0.00 & 2.39 & 7.08 & 0.00 & 0.00 & 0.00 & 0.00 & 0.00 & 0.00 & 0.00 & 6.51 \\
\hline
\end{tabular} \begin{tabular}{|l|l|l|l|l|l|l|l|l|l|l|l|l|l|l|l|l|}
\hline 10.53 & 0.00 & 0.00 & 0.00 & 0.00 & 0.00 & 0.00 & 0.00 & 1.53 & 0.00 & 0.00 & 0.00 & 0.00 & 0.00 & 0.00 & 0.00 & 0.00 \\
\hline
\end{tabular} \begin{tabular}{|l|l|l|l|l|l|l|l|l|l|l|l|l|l|l|l|l|}
\hline 3.59 & 0.00 & 0.00 & 0.00 & 0.00 & 0.00 & 0.00 & 0.00 & 0.00 & 1.86 & 0.00 & 0.00 & 0.00 & 0.00 & 0.00 & 1.41 & 0.00 \\
\hline
\end{tabular}

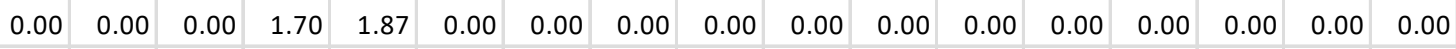

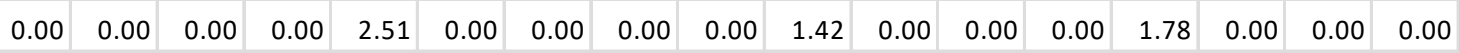




\section{Cs-134 Input R matrix}

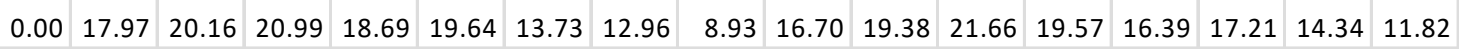
\begin{tabular}{|l|l|l|l|l|l|l|l|l|l|l|l|l|l|l|l|l|}
0.00 & 25.58 & 25.22 & 22.63 & 24.08 & 26.17 & 20.42 & 15.21 & 13.50 & 18.27 & 21.89 & 23.58 & 25.24 & 22.48 & 21.79 & 14.93 & 15.93 \\
\hline
\end{tabular} \begin{tabular}{|l|l|l|l|l|l|l|l|l|l|l|l|l|l|l|l|l|}
17.76 & 20.91 & 22.63 & 26.10 & 22.14 & 20.75 & 22.84 & 21.51 & 15.31 & 18.04 & 25.77 & 27.66 & 22.78 & 19.18 & 24.69 & 21.64 & 17.14 \\
\hline
\end{tabular}

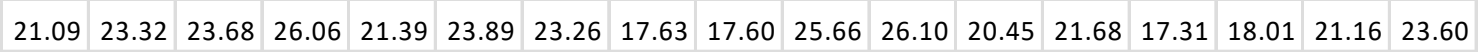
\begin{tabular}{|l|l|l|l|l|l|l|l|l|l|l|l|l|l|l|l|l|l|}
26.00 & 18.11 & 18.08 & 20.56 & 16.10 & 17.82 & 17.25 & 13.89 & 18.43 & 22.80 & 17.67 & 16.84 & 21.30 & 19.58 & 18.23 & 20.60 & 15.59 \\
\hline
\end{tabular} \begin{tabular}{|l|l|l|l|l|l|l|l|l|l|l|l|l|l|l|l|l|l|}
\hline 28.48 & 20.10 & 17.05 & 17.96 & 16.49 & 15.76 & 12.22 & 15.07 & 18.77 & 16.38 & 13.28 & 16.23 & 16.00 & 14.53 & 16.36 & 16.66 & 17.81 \\
\hline
\end{tabular}

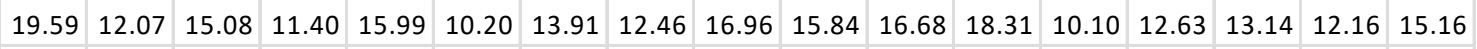
\begin{tabular}{|l|l|l|l|l|l|l|l|l|l|l|l|l|l|l|l|l|}
\hline 22.40 & 15.45 & 14.50 & 16.42 & 11.84 & 13.32 & 11.82 & 12.06 & 13.77 & 16.33 & 12.82 & 11.55 & 12.10 & 14.65 & 15.20 & 12.18 & 14.72 \\
\hline
\end{tabular}

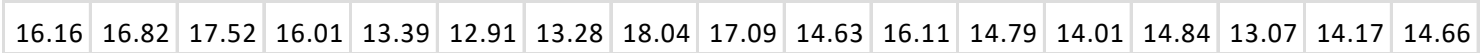
\begin{tabular}{|l|l|l|l|l|l|l|l|l|l|l|l|l|l|l|l|l|}
\hline 14.67 & 14.05 & 15.49 & 15.22 & 12.05 & 14.77 & 12.56 & 15.98 & 16.81 & 10.82 & 12.62 & 18.24 & 13.34 & 13.22 & 14.38 & 15.89 & 13.80 \\
\hline
\end{tabular}

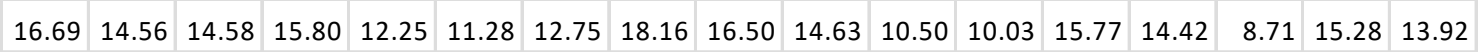
\begin{tabular}{|l|l|l|l|l|l|l|l|l|l|l|l|l|l|l|l|l|l|}
13.87 & 15.56 & 12.45 & 17.23 & 13.90 & 15.61 & 12.99 & 13.08 & 13.92 & 15.91 & 13.27 & 14.78 & 12.00 & 14.55 & 15.76 & 11.67 & 12.35 \\
\hline
\end{tabular} \begin{tabular}{|l|l|l|l|l|l|l|l|l|l|l|l|l|l|l|l|l|}
\hline 16.10 & 17.62 & 15.06 & 18.71 & 18.48 & 20.00 & 20.77 & 19.06 & 17.21 & 18.40 & 16.02 & 19.87 & 19.22 & 14.89 & 16.86 & 15.46 & 16.78 \\
\hline
\end{tabular} \begin{tabular}{|l|l|l|l|l|l|l|l|l|l|l|l|l|l|l|l|l|}
\hline 13.95 & 16.35 & 16.80 & 20.92 & 20.34 & 26.21 & 18.25 & 19.73 & 21.43 & 18.72 & 19.81 & 23.41 & 18.01 & 22.44 & 22.12 & 20.28 & 20.57 \\
\hline
\end{tabular} \begin{tabular}{|l|l|l|l|l|l|l|l|l|l|l|l|l|l|l|l|l|}
15.84 & 18.59 & 15.62 & 13.06 & 20.82 & 23.49 & 19.72 & 23.01 & 24.14 & 15.47 & 19.62 & 20.74 & 17.89 & 17.43 & 17.41 & 23.37 & 27.01 \\
\hline
\end{tabular}

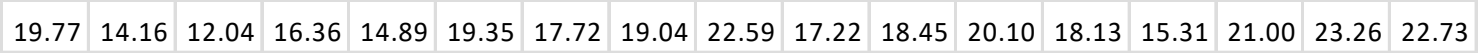

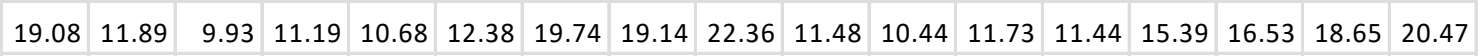

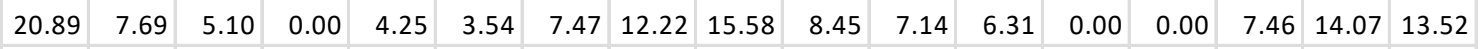

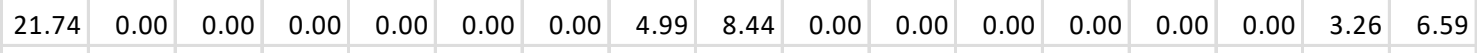

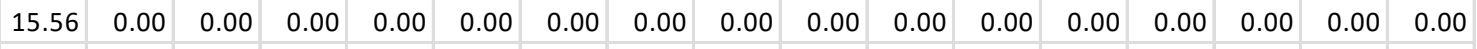

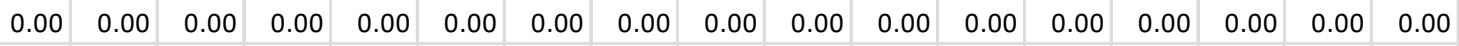

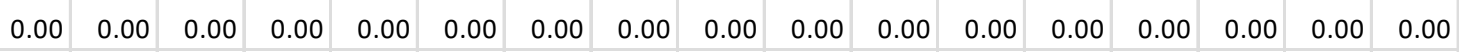

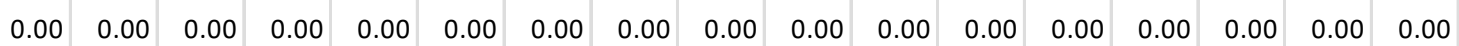




\section{Inner Ring 8 Axial Top}

Angles for Middle IR-08 Scan

\section{$\begin{array}{lllllllll}0.1 & 22.5 & 45 & 67.6 & 90 & 112.5 & 134.9 & 157.5 & 180\end{array}$}

Cs-137 Input R matrix

\begin{tabular}{|c|c|c|c|c|c|c|c|c|}
\hline 0.00 & 20.84 & 21.00 & 16.58 & 18.79 & 16.15 & 17.20 & 8.71 & 4.97 \\
\hline 2.21 & 19.09 & 21.68 & 22.33 & 26.13 & 18.65 & 19.96 & 21.19 & 15.58 \\
\hline 13.79 & 23.95 & 23.12 & 25.55 & 25.03 & 24.88 & 22.35 & 20.50 & 16.75 \\
\hline 17.64 & 27.07 & 24.37 & 22.02 & 22.97 & 26.39 & 25.50 & 23.50 & 22.72 \\
\hline 17.02 & 23.53 & 20.00 & 19.47 & 15.90 & 15.48 & 20.05 & 22.13 & 20.46 \\
\hline 25.18 & 16.71 & 21.27 & 15.82 & 16.75 & 14.47 & 18.68 & 17.55 & 18.67 \\
\hline 20.48 & 17.28 & 16.00 & 12.62 & 13.89 & 13.02 & 15.83 & 11.63 & 18.34 \\
\hline 16.52 & 18.82 & 13.61 & 14.94 & 12.90 & 13.89 & 12.99 & 14.07 & 10.29 \\
\hline 19.45 & 12.38 & 14.78 & 16.25 & 12.66 & 11.82 & 14.67 & 14.64 & 14.24 \\
\hline 14.91 & 15.39 & 15.55 & 13.12 & 10.01 & 12.50 & 14.62 & 12.37 & 14.19 \\
\hline 15.11 & 11.62 & 12.05 & 16.70 & 12.01 & 13.37 & 13.87 & 13.05 & 15.41 \\
\hline 12.38 & 13.23 & 14.27 & 17.20 & 14.44 & 11.91 & 16.79 & 18.13 & 14.84 \\
\hline 12.76 & 15.98 & 14.71 & 18.65 & 21.82 & 23.33 & 17.03 & 18.63 & 18.87 \\
\hline 12.22 & 17.46 & 17.51 & 20.32 & 18.64 & 22.67 & 17.64 & 19.33 & 19.64 \\
\hline 15.48 & 23.29 & 21.67 & 17.66 & 21.45 & 23.20 & 25.50 & 20.23 & 16.24 \\
\hline 21.21 & 21.19 & 14.49 & 15.98 & 20.60 & 21.37 & 21.12 & 19.34 & 26.25 \\
\hline 21.89 & 17.28 & 13.60 & 15.21 & 15.06 & 15.52 & 16.33 & 15.68 & 19.04 \\
\hline 21.37 & 9.50 & 5.01 & 4.93 & 5.54 & 11.07 & 9.69 & 13.25 & 15.87 \\
\hline 16.34 & 1.96 & 0.00 & 0.00 & 0.00 & 0.00 & 0.00 & 4.31 & 7.67 \\
\hline 11.91 & 0.00 & 0.00 & 0.00 & 0.00 & 1.65 & 0.00 & 0.00 & 0.00 \\
\hline 4.68 & 0.00 & 0.00 & 0.00 & 0.00 & 0.00 & 0.00 & 0.00 & 0.00 \\
\hline 0.00 & 0.00 & 0.00 & 0.00 & 0.00 & 0.00 & 0.00 & 0.00 & 0.00 \\
\hline 0.00 & 0.00 & 0.00 & 0.00 & 0.00 & 0.00 & 0.00 & 0.00 & 0.00 \\
\hline
\end{tabular}




\section{Cs-134 Input R matrix}

\begin{tabular}{|c|c|c|c|c|c|c|c|c|}
\hline 0.00 & 13.20 & 16.83 & 17.76 & 22.78 & 17.33 & 17.50 & 14.90 & 7.01 \\
\hline 0.00 & 21.39 & 19.84 & 23.60 & 21.47 & 23.13 & 24.08 & 15.85 & 16.18 \\
\hline 11.42 & 23.34 & 25.70 & 27.26 & 26.52 & 27.28 & 20.74 & 23.72 & 20.09 \\
\hline 17.15 & 26.25 & 24.71 & 24.31 & 20.29 & 25.95 & 22.10 & 23.05 & 22.84 \\
\hline 19.72 & 23.18 & 20.23 & 16.42 & 19.09 & 17.90 & 17.80 & 25.79 & 16.86 \\
\hline 25.11 & 21.20 & 13.12 & 16.85 & 18.89 & 14.95 & 16.92 & 16.19 & 14.85 \\
\hline 19.93 & 18.66 & 14.12 & 15.55 & 15.77 & 15.27 & 16.13 & 14.73 & 15.90 \\
\hline 13.63 & 18.94 & 12.85 & 18.08 & 15.92 & 13.19 & 15.47 & 14.80 & 19.02 \\
\hline 16.52 & 18.37 & 12.92 & 15.81 & 11.44 & 15.97 & 12.50 & 15.48 & 15.78 \\
\hline 15.70 & 16.11 & 13.34 & 17.59 & 17.22 & 10.18 & 16.46 & 15.08 & 16.17 \\
\hline 18.82 & 13.96 & 11.76 & 13.58 & 13.30 & 12.92 & 12.24 & 13.50 & 14.54 \\
\hline 16.66 & 16.30 & 18.77 & 15.40 & 16.50 & 14.31 & 17.55 & 15.58 & 16.64 \\
\hline 13.20 & 15.32 & 15.12 & 13.70 & 20.12 & 17.93 & 15.05 & 15.61 & 14.71 \\
\hline 10.27 & 19.71 & 23.74 & 24.14 & 22.50 & 23.39 & 20.08 & 20.21 & 18.49 \\
\hline 12.30 & 17.65 & 21.89 & 23.41 & 21.49 & 22.14 & 22.38 & 22.84 & 21.51 \\
\hline 16.26 & 17.45 & 18.58 & 17.22 & 21.05 & 19.50 & 23.09 & 19.41 & 28.82 \\
\hline 22.76 & 15.65 & 11.93 & 17.69 & 14.12 & 16.14 & 15.46 & 18.88 & 21.26 \\
\hline 18.25 & 10.11 & 8.86 & 3.84 & 8.93 & 10.10 & 13.34 & 16.44 & 15.03 \\
\hline 16.09 & 0.00 & 0.00 & 0.00 & 0.00 & 0.00 & 0.00 & 6.70 & 8.23 \\
\hline 13.58 & 0.00 & 0.00 & 0.00 & 0.00 & 0.00 & 0.00 & 0.00 & 0.00 \\
\hline 0.00 & 0.00 & 0.00 & 0.00 & 0.00 & 0.00 & 0.00 & 0.00 & 0.00 \\
\hline 0.00 & 0.00 & 0.00 & 0.00 & 0.00 & 0.00 & 0.00 & 0.00 & 0.00 \\
\hline 0.00 & 0.00 & 0.00 & 0.00 & 0.00 & 0.00 & 0.00 & 0.00 & 0.00 \\
\hline
\end{tabular}




\section{Inner Ring 10 Axial Middle}

Angles for Middle IR-10 Scan

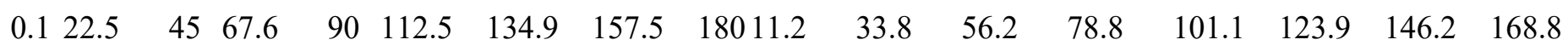

Cs-137 Input R matrix

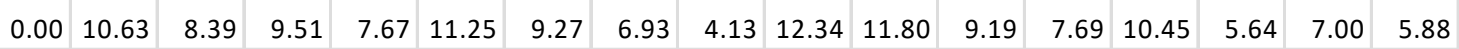
\begin{tabular}{|l|l|l|l|l|l|l|l|l|l|l|l|l|l|l|l|l|l|l|}
\hline 2.86 & 16.31 & 16.50 & 19.38 & 16.48 & 16.50 & 13.46 & 10.83 & 8.75 & 13.29 & 16.77 & 16.38 & 16.61 & 16.24 & 9.61 & 11.65 & 7.53 \\
\hline
\end{tabular} \begin{tabular}{|l|l|l|l|l|l|l|l|l|l|l|l|l|l|l|l|l|}
8.07 & 22.26 & 21.27 & 22.06 & 22.43 & 22.05 & 18.15 & 11.37 & 10.42 & 17.16 & 20.98 & 20.22 & 20.55 & 22.15 & 19.92 & 15.30 & 13.46 \\
\hline
\end{tabular}

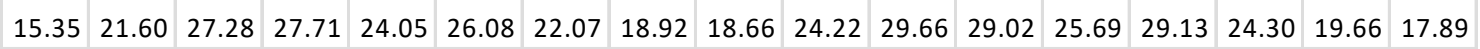

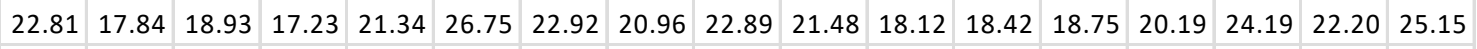
\begin{tabular}{|l|l|l|l|l|l|l|l|l|l|l|l|l|l|l|l|l|l|}
28.61 & 16.11 & 16.16 & 14.75 & 19.14 & 16.95 & 15.76 & 20.10 & 18.73 & 16.35 & 13.95 & 14.75 & 15.92 & 15.11 & 16.22 & 17.08 & 18.44 \\
\hline
\end{tabular} \begin{tabular}{|l|l|l|l|l|l|l|l|l|l|l|l|l|l|l|l|l|}
\hline 16.95 & 11.90 & 11.55 & 14.46 & 15.76 & 16.05 & 16.53 & 14.78 & 12.16 & 15.94 & 14.38 & 13.65 & 15.81 & 15.43 & 17.30 & 12.20 & 16.03 \\
\hline
\end{tabular} \begin{tabular}{|l|l|l|l|l|l|l|l|l|l|l|l|l|l|l|l|l|}
\hline 15.14 & 13.99 & 13.76 & 13.04 & 12.05 & 14.55 & 14.40 & 15.00 & 16.92 & 17.62 & 15.44 & 14.72 & 11.66 & 10.93 & 12.47 & 14.53 & 14.62 \\
\hline
\end{tabular}

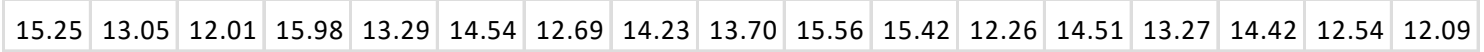

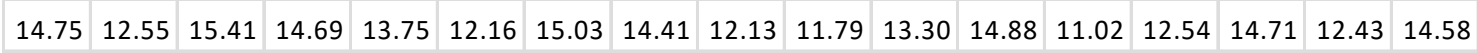

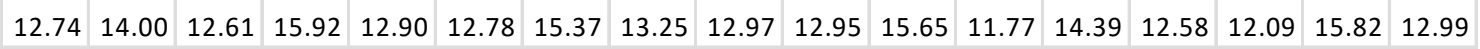
\begin{tabular}{|l|l|l|l|l|l|l|l|l|l|l|l|l|l|l|l|l|}
\hline 14.04 & 16.86 & 13.18 & 15.17 & 12.12 & 14.80 & 15.58 & 17.44 & 16.42 & 12.70 & 13.88 & 11.75 & 13.81 & 13.25 & 12.09 & 15.19 & 16.46 \\
\hline
\end{tabular}

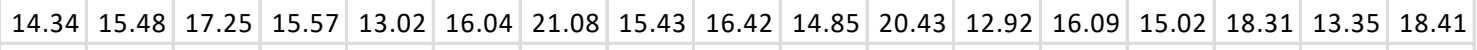

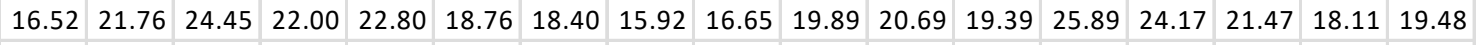
\begin{tabular}{|l|l|l|l|l|l|l|l|l|l|l|l|l|l|l|l|l|}
\hline 16.18 & 19.99 & 13.62 & 20.36 & 15.66 & 18.76 & 22.53 & 22.81 & 25.89 & 22.05 & 19.51 & 20.71 & 22.13 & 21.44 & 23.33 & 28.94 & 28.43 \\
\hline
\end{tabular} \begin{tabular}{|l|l|l|l|l|l|l|l|l|l|l|l|l|l|l|l|l|}
\hline 25.46 & 12.87 & 13.88 & 16.66 & 14.03 & 18.05 & 18.72 & 23.11 & 22.61 & 15.67 & 14.35 & 15.87 & 12.24 & 15.63 & 17.34 & 21.87 & 25.04 \\
\hline
\end{tabular}

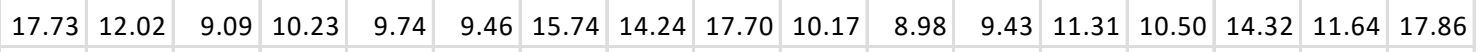

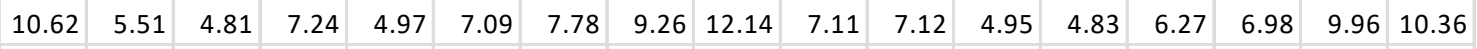

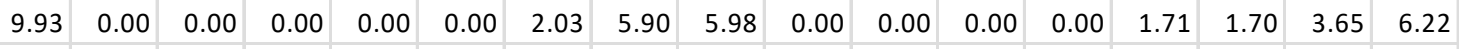

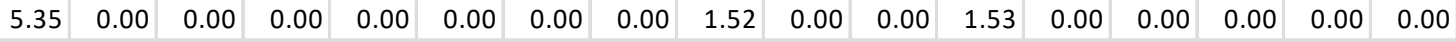

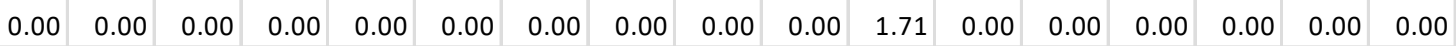

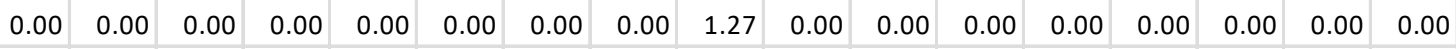

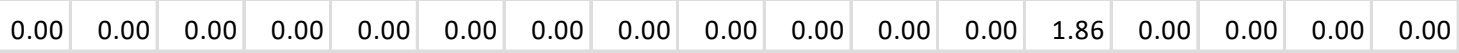




\section{Cs-134 Input R matrix}

\begin{tabular}{|l|l|l|l|l|l|l|l|l|l|l|l|l|l|l|l|l|}
\hline 0.00 & 8.84 & 9.62 & 9.83 & 9.11 & 10.21 & 7.68 & 5.04 & 4.98 & 6.79 & 11.54 & 10.62 & 5.39 & 6.93 & 5.61 & 4.41 & 5.05 \\
\hline
\end{tabular} \begin{tabular}{|l|l|l|l|l|l|l|l|l|l|l|l|l|l|l|l|l|}
0.00 & 18.15 & 14.68 & 13.30 & 17.27 & 11.85 & 9.76 & 7.89 & 10.51 & 13.59 & 17.02 & 16.01 & 12.35 & 12.27 & 10.52 & 7.59 & 0.00 \\
\hline
\end{tabular} \begin{tabular}{|l|l|l|l|l|l|l|l|l|l|l|l|l|l|l|l|l|l|l|}
6.92 & 20.79 & 23.98 & 17.40 & 20.25 & 13.44 & 17.22 & 9.00 & 8.62 & 17.61 & 20.28 & 22.01 & 19.77 & 15.80 & 15.87 & 12.73 & 12.77 \\
\hline
\end{tabular} \begin{tabular}{|l|l|l|l|l|l|l|l|l|l|l|l|l|l|l|l|l|}
13.60 & 22.32 & 23.84 & 22.50 & 21.55 & 21.20 & 17.89 & 13.25 & 14.74 & 22.69 & 20.53 & 21.27 & 25.17 & 27.76 & 21.07 & 17.04 & 16.69 \\
\hline
\end{tabular} \begin{tabular}{|l|l|l|l|l|l|l|l|l|l|l|l|l|l|l|l|l|l|l|l|l|l|}
19.49 & 17.70 & 16.72 & 17.77 & 18.58 & 19.51 & 18.08 & 17.89 & 19.56 & 19.75 & 14.02 & 18.17 & 18.63 & 19.51 & 19.00 & 18.66 & 19.60 \\
\hline
\end{tabular} \begin{tabular}{|l|l|l|l|l|l|l|l|l|l|l|l|l|l|l|l|l|}
\hline 22.55 & 12.48 & 14.81 & 14.96 & 15.90 & 14.64 & 15.94 & 14.53 & 16.67 & 14.46 & 15.37 & 12.52 & 16.80 & 14.34 & 15.34 & 22.01 & 19.61 \\
\hline
\end{tabular} \begin{tabular}{|l|l|l|l|l|l|l|l|l|l|l|l|l|l|l|l|l|}
15.68 & 13.97 & 12.45 & 12.96 & 14.73 & 15.34 & 11.25 & 15.09 & 21.51 & 13.58 & 13.72 & 12.96 & 14.26 & 13.81 & 14.04 & 13.48 & 12.07 \\
\hline
\end{tabular} \begin{tabular}{|l|l|l|l|l|l|l|l|l|l|l|l|l|l|l|l|l|}
12.11 & 10.22 & 10.91 & 10.18 & 14.79 & 10.21 & 13.18 & 11.91 & 11.94 & 13.25 & 11.46 & 12.86 & 10.24 & 12.49 & 9.82 & 10.57 & 13.51 \\
\hline
\end{tabular} \begin{tabular}{|l|l|l|l|l|l|l|l|l|l|l|l|l|l|l|l|l|}
\hline 12.27 & 12.63 & 12.37 & 12.37 & 13.67 & 10.96 & 15.62 & 8.55 & 11.70 & 12.91 & 12.21 & 11.92 & 10.22 & 10.95 & 15.56 & 10.64 & 12.70 \\
\hline
\end{tabular}

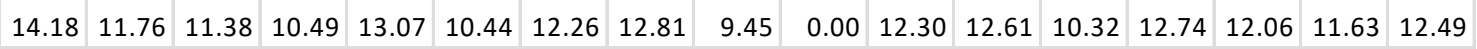
\begin{tabular}{|l|l|l|l|l|l|l|l|l|l|l|l|l|l|l|l|l|l|}
\hline 9.15 & 11.59 & 10.65 & 12.03 & 14.01 & 10.87 & 10.93 & 13.40 & 12.74 & 8.72 & 13.91 & 10.42 & 10.71 & 11.36 & 10.46 & 12.54 & 15.72 \\
\hline
\end{tabular} \begin{tabular}{|l|l|l|l|l|l|l|l|l|l|l|l|l|l|l|l|l|}
\hline 10.95 & 13.38 & 14.06 & 12.51 & 12.78 & 13.09 & 13.10 & 13.21 & 12.85 & 8.66 & 14.52 & 6.81 & 13.40 & 13.71 & 12.23 & 13.89 & 12.57 \\
\hline
\end{tabular} \begin{tabular}{|l|l|l|l|l|l|l|l|l|l|l|l|l|l|l|l|l|}
\hline 11.06 & 14.49 & 12.51 & 13.80 & 12.24 & 15.36 & 10.42 & 14.47 & 15.76 & 15.72 & 12.00 & 13.44 & 13.74 & 10.34 & 16.65 & 14.12 & 15.54 \\
\hline
\end{tabular}

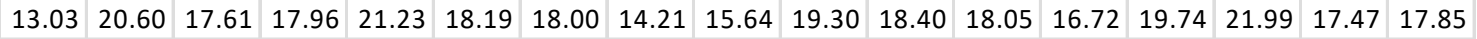

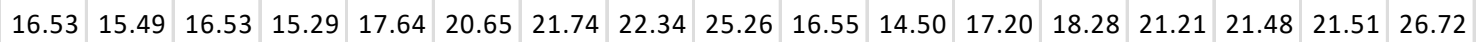
\begin{tabular}{|l|l|l|l|l|l|l|l|l|l|l|l|l|l|l|l|l|}
\hline 20.70 & 10.69 & 8.21 & 12.96 & 10.73 & 17.92 & 17.82 & 16.50 & 23.50 & 11.27 & 14.13 & 12.54 & 12.78 & 14.60 & 16.05 & 18.89 & 17.27 \\
\hline
\end{tabular}

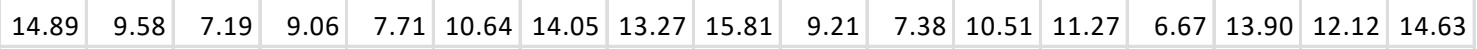

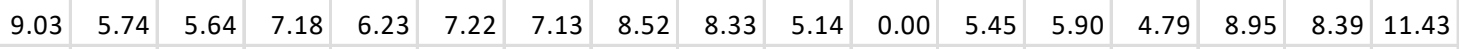
\begin{tabular}{|l|l|l|l|l|l|l|l|l|l|l|l|l|l|l|l|l|}
\hline 7.97 & 0.00 & 0.00 & 0.00 & 0.00 & 3.79 & 3.55 & 3.70 & 4.83 & 0.00 & 0.00 & 0.00 & 0.00 & 0.00 & 0.00 & 4.38 & 6.76 \\
\hline
\end{tabular} \begin{tabular}{|l|l|l|l|l|l|l|l|l|l|l|l|l|l|l|l|l|}
\hline 4.41 & 0.00 & 0.00 & 0.00 & 0.00 & 0.00 & 0.00 & 0.00 & 0.00 & 0.00 & 0.00 & 0.00 & 0.00 & 0.00 & 0.00 & 0.00 & 0.00 \\
\hline
\end{tabular}

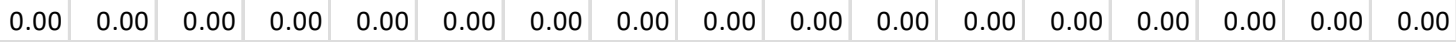

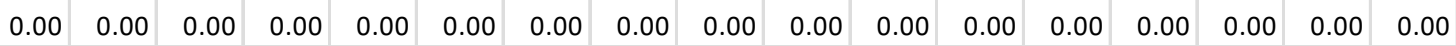

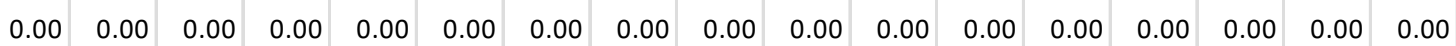




\section{Ag-110m Input R matrix}

\begin{tabular}{|c|c|c|c|c|c|c|c|c|c|c|c|c|c|c|c|c|}
\hline 0.00 & .32 & 5.34 & 3.82 & 4.97 & 2.91 & & & & & 0.00 & 17.16 & 18.67 & 6.33 & חم & .00 & \\
\hline & & & & & & & & & & & & & & & & \\
\hline & & & & & & & & & & & & & & & & 0.18 \\
\hline & & & & & & & & & & 1.05 & & & & .00 & & 16.25 \\
\hline & & & & & & 10.08 & & & & & & & & 0.00 & & 12.12 \\
\hline & & & & & & & & & & & & .00 & & 00 & & .00 \\
\hline & & & & & & & & & & & & & & & & 0.00 \\
\hline & & & & & & 1 & 0.00 & & & 9.16 & & 0.00 & & & & 174 \\
\hline & & & & & & & & & & & & 0.00 & 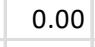 & & & 2.26 \\
\hline & & & & & & & & & & & & & & & & $0 \Omega$ \\
\hline & & & & & & & & & & & & & & & & \\
\hline 5.22 & & & & & & & & & & & & & & & & \\
\hline 2.82 & & & & & & 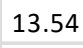 & & & & & & & & & & .00 \\
\hline & & & & & & & & & & & & & 0.95 & & & \\
\hline 4.67 & 3.11 & & & & & & 3.24 & & & 00 & & & 0 & .00 & & 0.44 \\
\hline & & & & & & & & & & & & & & & & \\
\hline 16.40 & 3.70 & & & & & 20.14 & 11.33 & & & & & 0.00 & 5.95 & & & 6.25 \\
\hline & & & & & & & & & & & & & & & & \\
\hline 1.96 & & & & & & & & & & & & & & & & .00 \\
\hline & & & & & & & & & & & & & & & & 0.00 \\
\hline & & & & & & & & & & & & & & 00 & & . \\
\hline 0.00 & & & & & & & & & & & & & . & & 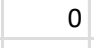 & \\
\hline 0.00 & 0.00 & 0.00 & 0.00 & 0.00 & 0.00 & 0.00 & 0.00 & & 0.00 & 0.00 & 0.00 & & & & & \\
\hline
\end{tabular}




\section{Inner Ring 10 Axial Top}

Angles for Upper IR-10 Scan

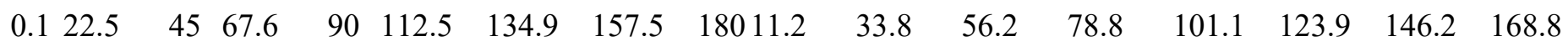

\section{Cs-137 Input R matrix}

\begin{tabular}{|c|c|c|c|c|c|c|c|c|c|c|c|c|c|c|c|c|}
\hline .00 & & 67 & & & .79 & & .81 & 3.26 & 60 & 1 & & .25 & .40 & 5.19 & .13 & \\
\hline & & & & & & & & & & & & & & & & \\
\hline 3.98 & & & & & & & & & & & & & & & & \\
\hline & & & & & & & & & & .32 & & & 8.65 & & & 3.8 \\
\hline & & & & & & & & 7.03 & & & & & & & & 637 \\
\hline .2 & & & & & & & & 0.31 & & & & & & & 38 & 2.1 \\
\hline 2.18 & & & & & & & & 3 & & 9.85 & & & & & & \\
\hline & & & & & & & & & & & & & & & & 98 \\
\hline 9.38 & & & & & & & .23 & 10.66 & & 9.01 & & & 9.83 & & & 80 \\
\hline & & & & & & & & & & .94 & & & & & .92 & 0.51 \\
\hline 1.01 & & & & & & & & & & & & & & & & \\
\hline .39 & & & & & & & & & & 26 & & & & & & 75 \\
\hline .94 & & & & & & & & & & & & & & & & \\
\hline & & & & & & & & & & & & & & & & 178 \\
\hline 1.02 & & & & & & & & 16.94 & & & & & & & & \\
\hline .63 & & & & & 5.89 & & 5.97 & & & 0.80 & & & 70 & & & \\
\hline .56 & & & & & & & 1.88 & 12.06 & & & & & .79 & & 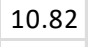 & 1.42 \\
\hline & & & & & & & & & & & & & & & & \\
\hline 0.53 & & & & & & & & & & & & & & & & . \\
\hline 4.40 & & & & & & & & & & & & & & & & \\
\hline & & & & & & & & & & & & & & & & .00 \\
\hline 0.00 & & & & & & & & & & & & & & & & 0.00 \\
\hline 0.00 & 00 & .00 & .00 & 1.61 & .00 & 0.00 & .00 & 0.00 & .00 & 0.00 & .65 & 0.00 & 0.00 & 1.47 & 0.00 & 1.34 \\
\hline
\end{tabular}




\section{Cs-134 Input R matrix}

\begin{tabular}{|c|c|c|c|c|c|c|c|c|c|c|c|c|c|c|c|c|}
\hline 00 & & & & & & & & & & & & & 8.81 & & .00 & \\
\hline & & & & & & & & & & & & & & & & \\
\hline & & & & & & & & & & & & & & & & \\
\hline & & & & & & & & & & & & & & & & \\
\hline & & & & & & & & & & & & & & & & 10.95 \\
\hline & & & & & & & & & & & & & & & & 8 \\
\hline & & & & & & & & & & & & & & & & f \\
\hline & & & & & & & & & & & & & & & & 15 \\
\hline & & & & & & & & & & & & & & & & \\
\hline & & & & & & & & & & & & & & & & \\
\hline & & & & & & & & & & & & & & & & \\
\hline & & & & & & & & & & & & & & & & \\
\hline & & & & & & & & & & & & & & & & \\
\hline & & & & & & & & & & & & & & & & 77 \\
\hline & & & & .16 & & & & & & & 0.89 & & & & & 0.1 \\
\hline & & & & & & & & & & & & & & & & \\
\hline & & & & & & & & & & & & & & & & 11.75 \\
\hline & & & & & & & & & & & & & & & & \\
\hline & & & & & & & & & & & & & & & & \\
\hline & & & & & & & & & & & & & & & & \\
\hline & & & & & & & & & & & & & & & & \\
\hline 0.00 & & & & & & & & & & & 0.00 & & & 0.00 & & 0.00 \\
\hline & & 00 & & 00 & .00 & .00 & .00 & .00 & & 0.00 & 0.00 & $0.0 c$ & 0.00 & 0.00 & 0.00 & 0.0 \\
\hline
\end{tabular}




\section{Ag-110m Input R matrix}

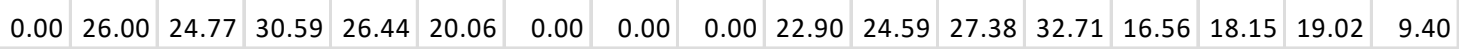
\begin{tabular}{|l|l|l|l|l|l|l|l|l|l|l|l|l|l|l|l|l|l|}
0.00 & 39.36 & 34.95 & 30.37 & 28.25 & 31.84 & 30.83 & 23.30 & 15.20 & 30.71 & 31.26 & 31.81 & 24.01 & 30.34 & 30.03 & 22.59 & 17.34 \\
\hline
\end{tabular} \begin{tabular}{|l|l|l|l|l|l|l|l|l|l|l|l|l|l|l|l|l|}
23.12 & 18.38 & 29.96 & 22.73 & 31.57 & 34.22 & 35.53 & 25.11 & 20.69 & 21.52 & 29.21 & 28.57 & 26.57 & 25.60 & 33.51 & 35.15 & 19.91 \\
\hline
\end{tabular} \begin{tabular}{|l|l|l|l|l|l|l|l|l|l|l|l|l|l|l|l|l|}
\hline 23.84 & 0.00 & 19.20 & 18.72 & 22.17 & 20.05 & 20.90 & 18.75 & 33.41 & 20.95 & 20.63 & 15.45 & 28.01 & 16.70 & 17.45 & 34.56 & 17.51 \\
\hline
\end{tabular} \begin{tabular}{|l|l|l|l|l|l|l|l|l|l|l|l|l|l|l|l|l|l|}
\hline 22.51 & 12.82 & 12.71 & 15.26 & 12.74 & 13.69 & 19.21 & 19.87 & 22.05 & 16.59 & 18.14 & 15.21 & 15.07 & 17.38 & 13.62 & 0.00 & 0.00 \\
\hline
\end{tabular} \begin{tabular}{|l|l|l|l|l|l|l|l|l|l|l|l|l|l|l|l|l|}
\hline 18.91 & 14.04 & 25.50 & 16.64 & 13.97 & 12.60 & 15.22 & 13.93 & 19.23 & 0.00 & 14.53 & 13.95 & 22.67 & 11.89 & 11.36 & 15.12 & 18.20 \\
\hline
\end{tabular} \begin{tabular}{|l|l|l|l|l|l|l|l|l|l|l|l|l|l|l|l|l|}
\hline 17.67 & 0.00 & 0.00 & 0.00 & 16.39 & 17.38 & 20.56 & 13.68 & 0.00 & 16.78 & 13.34 & 13.25 & 13.44 & 0.00 & 16.13 & 16.18 & 18.53 \\
\hline
\end{tabular} \begin{tabular}{|l|l|l|l|l|l|l|l|l|l|l|l|l|l|l|l|l|}
\hline 21.57 & 14.41 & 15.44 & 19.86 & 0.00 & 15.84 & 18.75 & 18.09 & 19.32 & 0.00 & 13.63 & 0.00 & 12.37 & 12.73 & 16.98 & 0.00 & 13.51 \\
\hline
\end{tabular}

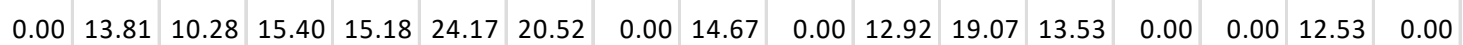
\begin{tabular}{|l|l|l|l|l|l|l|l|l|l|l|l|l|l|l|l|l|}
10.75 & 15.84 & 10.15 & 12.22 & 17.06 & 16.33 & 14.40 & 13.57 & 0.00 & 15.36 & 0.00 & 15.42 & 11.25 & 13.44 & 0.00 & 13.41 & 14.66 \\
\hline
\end{tabular} \begin{tabular}{|l|l|l|l|l|l|l|l|l|l|l|l|l|l|l|l|l|l|l|l|l|l|l|}
14.77 & 12.51 & 15.69 & 0.00 & 10.34 & 16.20 & 11.68 & 13.57 & 0.00 & 19.45 & 12.70 & 0.00 & 11.04 & 12.29 & 15.25 & 15.86 & 15.65 \\
\hline
\end{tabular}

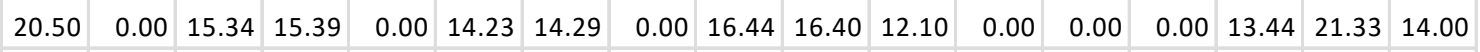
\begin{tabular}{|l|l|l|l|l|l|l|l|l|l|l|l|l|l|l|l|l|}
19.71 & 15.07 & 8.97 & 13.57 & 14.03 & 13.75 & 11.82 & 14.07 & 0.00 & 18.01 & 11.88 & 15.65 & 13.49 & 13.47 & 0.00 & 18.31 & 17.93 \\
\hline
\end{tabular} \begin{tabular}{|l|l|l|l|l|l|l|l|l|l|l|l|l|l|l|l|l|l|}
\hline 14.56 & 22.62 & 18.61 & 16.68 & 16.29 & 14.49 & 22.44 & 17.12 & 19.20 & 19.34 & 16.38 & 17.97 & 14.14 & 11.99 & 0.00 & 12.43 & 17.15 \\
\hline
\end{tabular}

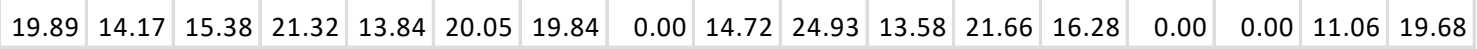

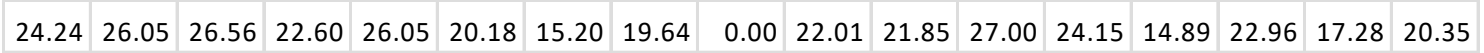

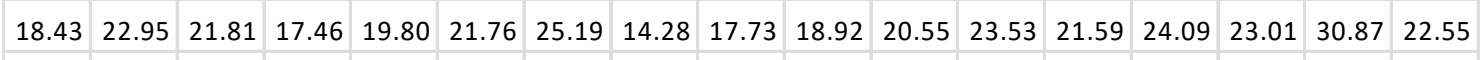

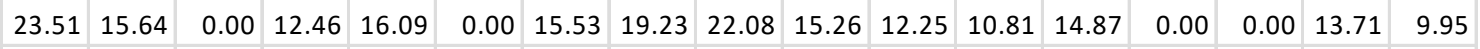

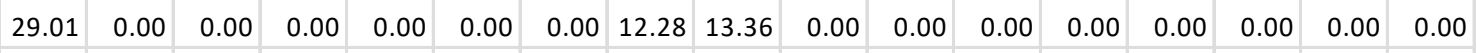

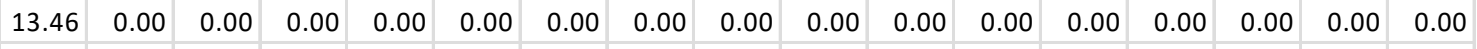

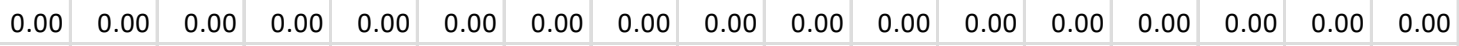

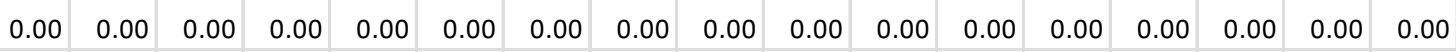

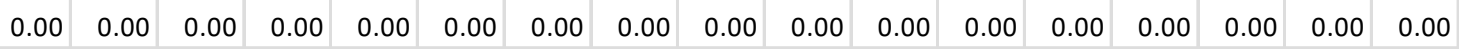




\section{Outer Ring 03 Axial Middle}

Angles for Middle OR-03 Scan

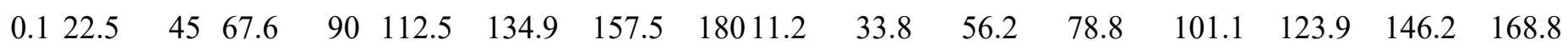

\section{Cs-137 Input R matrix}

\begin{tabular}{|c|c|c|c|c|c|c|c|c|c|c|c|c|c|c|c|c|}
\hline 0.00 & 0.00 & 0.00 & 0.00 & 0.00 & 0.00 & 0.00 & 0.00 & 0.00 & 0.00 & 0.00 & 0.00 & 0.00 & 0.00 & 0.00 & 0.00 & 0.00 \\
\hline 0.00 & 0.00 & 0.00 & 0.00 & 0.00 & 0.00 & 0.00 & 0.00 & 0.00 & 0.00 & 0.00 & 0.00 & 0.00 & 0.00 & 0.00 & 1.21 & 0.00 \\
\hline 0.00 & 0.00 & 0.00 & 0.00 & 0.00 & 0.00 & 0.00 & 0.00 & 0.00 & 0.00 & 0.00 & 0.00 & 0.00 & 0.00 & 0.00 & 0.00 & 0.00 \\
\hline 0.00 & 0.00 & 0.00 & 0.00 & 0.00 & .00 & 0.00 & 0.00 & 0.00 & 0.00 & 0.00 & 0.00 & 0.00 & 0.00 & 0.00 & 0.00 & 0.00 \\
\hline 0.00 & 1.14 & 0.00 & 0.00 & 0.00 & .00 & 0.00 & 0.00 & 0.00 & 0.00 & 0.00 & 0.00 & 0.00 & .00 & 0.00 & .00 & 0.00 \\
\hline 3.60 & 3.49 & 2.88 & 2.71 & 3.16 & 55 & 1.31 & 0.00 & 2.17 & 1.56 & 0.00 & 0.00 & 0. & .00 & 0.00 & & 1.91 \\
\hline 6.37 & 4.61 & 5.05 & 3.58 & 4.54 & .34 & 2.90 & 4.05 & 3.26 & 2.81 & 4.09 & 3.81 & 3.18 & 3.22 & 3.40 & 3.32 & 3.51 \\
\hline 8.90 & 6.68 & 8.97 & 6.64 & 8.17 & 3.41 & 7.52 & 4.71 & 6.33 & 8.16 & 5.67 & 7.72 & 5.20 & 5.03 & 4.77 & 5.25 & 8.58 \\
\hline 11.25 & 8.17 & 11.10 & 0.08 & 8.36 & 6.45 & 9.96 & 7.34 & 9.24 & 7.84 & 5.63 & 6.63 & 8.21 & 8.39 & 7.18 & 6.65 & 8.94 \\
\hline 6.45 & 9.13 & 6.26 & 6.85 & 7.98 & 8.40 & 8.63 & 13.25 & 8.50 & 9.75 & 8.40 & 7.77 & 10.24 & 9.93 & 13.31 & 8.74 & 8.48 \\
\hline 6.75 & 6.99 & 6.38 & 6.45 & 5.65 & 7.02 & 7.46 & 7.54 & 5.31 & 6.99 & 8.08 & 7.91 & 7.04 & 6.29 & 6.95 & 5.69 & 7.32 \\
\hline 6.41 & 5.06 & 6.41 & 6.00 & 6.11 & 6.41 & 4.58 & 5.81 & 5.73 & 6.26 & 5.22 & 7.24 & 6.32 & 5.27 & 6.76 & 6.58 & 6.87 \\
\hline 4.97 & 6.09 & 4.39 & 6.25 & 5.28 & 5.08 & 4.71 & 6.67 & 5.23 & 4.59 & 7.60 & 2.84 & 6.10 & 5.00 & 5.20 & 4.99 & 5.45 \\
\hline 4.77 & 3.80 & 5.23 & 5.59 & 5.62 & 4.09 & 4.55 & 6.09 & 5.95 & 3.87 & 6.03 & 5.73 & 5.41 & 3.29 & 4.89 & 3.82 & 4.06 \\
\hline 4.65 & 5.11 & 5.00 & 5.69 & 5.57 & 4.25 & 5.38 & 6.53 & 4.48 & 4.24 & 3.10 & 3.56 & 4.23 & 4.24 & 2.04 & 4.99 & 3.36 \\
\hline 3.75 & 3.52 & 3.52 & 3.70 & 3.80 & 3.35 & 5.03 & 5.15 & 4.59 & 4.42 & 3.25 & 4.39 & 5.43 & 2.01 & 4.57 & 5.00 & 4.12 \\
\hline 6.28 & 2.70 & 4.12 & 5.56 & 4.74 & 1.26 & 3.20 & 3.30 & 3.82 & 3.45 & 4.39 & 4.59 & 3.82 & 4.98 & 3.14 & 3.98 & 3.40 \\
\hline 4.92 & 3.71 & 2.88 & 4.52 & 4.09 & .73 & 5.85 & 3.39 & 2.69 & 3.74 & 3.58 & 4.15 & 3.97 & 3.75 & 2.41 & 5.20 & 2.25 \\
\hline 3.77 & 4.94 & 5.65 & 4.61 & 5.24 & 0.00 & 4.50 & 2.66 & 5.31 & 5.16 & 3.91 & 4.55 & 3.57 & 4.77 & 4.78 & 5.39 & 5.46 \\
\hline 5.20 & 5.43 & 4.57 & 3.84 & 3.83 & 3.85 & 4.12 & 3.68 & 4.88 & 2.87 & 3.67 & 4.17 & 3.58 & 3.96 & 4.02 & 4.43 & 4.47 \\
\hline 4.26 & 5.40 & 6.35 & 4.94 & 4.93 & 1.88 & 4.65 & 1.91 & 4.01 & 5.32 & 5.17 & 5.10 & 2.98 & 3.55 & 3.43 & 4.36 & 4.67 \\
\hline 4.02 & 5.71 & 4.64 & 4.31 & 4.59 & 3.79 & 6.67 & 3.63 & 3.77 & 6.94 & 4.33 & 2.32 & 5.05 & 7.23 & 5.06 & 3.27 & 3.38 \\
\hline 5.95 & 3.02 & 4.45 & 5.25 & 4.45 & 6.41 & 4.95 & 4.90 & 4.32 & 4.73 & 6.03 & 3.83 & 2.88 & 3.91 & 5.16 & 5.39 & 4.33 \\
\hline 5.66 & 4.90 & 5.09 & 4.34 & 4.68 & 5.92 & 4.65 & 6.17 & 4.59 & 5.23 & 5.47 & 3.88 & 4.41 & 3.42 & 4.48 & 4.15 & 4.22 \\
\hline 5.91 & 6.99 & 4.37 & 3.48 & 5.02 & 4.03 & 7.42 & 4.95 & 3.79 & 4.16 & 3.44 & 4.08 & 4.33 & 4.47 & 4.26 & 5.84 & 5.28 \\
\hline 7.99 & 6.94 & 5.47 & 5.39 & 7.27 & 6.59 & 8.17 & 6.57 & 5.41 & 3.95 & 5.92 & 5.87 & 6.29 & 5.95 & 5.24 & 5.30 & 5.58 \\
\hline 6.55 & 9.42 & 9.09 & 9.13 & 7.64 & 7.09 & 7.91 & 5.79 & 7.56 & 5.90 & 6.19 & 6.79 & 5.80 & 4.21 & 3.36 & 7.20 & 8.34 \\
\hline 8.65 & 11.17 & 12.24 & 1.31 & 10.39 & 8.60 & 8.41 & 6.58 & 9.11 & 7.72 & 9.22 & 8.28 & 8.53 & 8.06 & 7.71 & 7.07 & 8.95 \\
\hline 9.56 & 8.48 & 8.46 & 10.06 & 9.02 & 7.77 & 13.17 & 11.29 & 10.66 & 9.27 & 10.68 & 9.09 & 11.22 & 10.74 & 10.40 & 8.40 & 10.92 \\
\hline 5.60 & 4.55 & 6.06 & 7.32 & 6.67 & 7.55 & 5.46 & 8.60 & 8.28 & 9.92 & 7.39 & 6.89 & 6.91 & 6.82 & 7.50 & 9.26 & 9.69 \\
\hline 6.11 & 2.85 & & 4.02 & 4.04 & 3.99 & 6.08 & 5.43 & 4.17 & 5.50 & 4.78 & 7.21 & 5.38 & 5.26 & 5.84 & 6.67 & 4.87 \\
\hline 0.00 & 1.43 & & 2.35 & 1.82 & 1.76 & 3.30 & 1.65 & 2.07 & 2.14 & 1.76 & 1.65 & 4.43 & 3.30 & 3.73 & 2.61 & 2.67 \\
\hline 0.00 & 0.00 & 0.00 & 0.00 & 0.00 & 0.00 & 0.00 & 0.00 & 0.00 & 1.32 & 0.00 & 0.00 & 0.00 & 0.00 & 0.00 & 0.00 & 0.00 \\
\hline 0.00 & 0.0 & $0.0 c$ & 0.00 & 0.00 & $0.0 c$ & 0.00 & 0.0 & 0.00 & 0.00 & 0.00 & 0.00 & 0.00 & 0.00 & 0.00 & 0.00 & 0.00 \\
\hline 0.00 & 0.00 & 0.00 & 0.00 & 0.00 & 0.00 & 0.00 & 0.00 & 0.00 & 0.00 & 0.00 & 0.00 & 1.22 & 0.00 & 0.00 & 0.00 & 0.00 \\
\hline 1.28 & 0.00 & 0.00 & 0.00 & 0.00 & 0.00 & 0.00 & 0.00 & 0.00 & 0.00 & 1.18 & 0.00 & 0.00 & 0.00 & 0.00 & 0.00 & 0.00 \\
\hline 0.00 & 1.32 & 0.00 & 0.00 & 0.00 & 0.00 & 0.00 & 0.00 & 0.00 & 0.00 & 0.00 & 1.26 & 0.00 & 0.00 & 0.00 & 0.00 & 0.00 \\
\hline
\end{tabular}




\section{Outer Ring 07 Axial Middle}

Angles for Middle OR-07 Scan
$0.122 .5 \quad 45 \quad 67.6$
$90 \quad 112.5$
$134.9 \quad 157.5 \quad 18011.2$
33.8
56.2
78.8
$\begin{array}{llll}101.1 & 123.9 & 146.2 & 168.8\end{array}$

\section{Cs-137 Input R matrix}

$\begin{array}{lllllllllllllllllll}0.00 & 0.00 & 0.00 & 0.00 & 0.00 & 0.00 & 0.00 & 0.00 & 0.00 & 0.00 & 0.00 & 0.00 & 0.00 & 0.00 & 0.00 & 0.00 & 0.00\end{array}$ $\begin{array}{llllllllllllllllll}0.00 & 0.00 & 0.00 & 0.00 & 0.00 & 0.00 & 0.00 & 0.00 & 0.00 & 0.00 & 0.00 & 0.00 & 0.00 & 0.00 & 0.00 & 0.00 & 0.00\end{array}$ $\begin{array}{llllllllllllllllllll}0.00 & 0.00 & 0.00 & 0.00 & 0.00 & 0.00 & 0.00 & 0.00 & 0.00 & 0.00 & 0.00 & 0.00 & 0.00 & 0.00 & 0.00 & 0.00 & 0.00\end{array}$ $\begin{array}{lllllllllllllllll}0.00 & 0.00 & 0.00 & 0.00 & 0.00 & 0.00 & 1.25 & 0.00 & 0.00 & 0.00 & 0.00 & 0.00 & 0.00 & 0.00 & 0.00 & 0.00 & 0.00\end{array}$ $\begin{array}{llllllllllllllllllll}1.78 & 2.15 & 0.00 & 0.00 & 2.24 & 2.37 & 2.12 & 1.91 & 0.00 & 2.39 & 2.54 & 2.67 & 0.00 & 1.68 & 2.33 & 0.00 & 2.33\end{array}$ $\begin{array}{lllllllllllllllllllll}3.21 & 3.17 & 2.51 & 3.12 & 3.21 & 3.10 & 1.78 & 3.77 & 4.51 & 4.76 & 4.18 & 1.99 & 4.01 & 3.13 & 4.04 & 2.51 & 2.95\end{array}$ $\begin{array}{llllllllllllllllllllll}3.44 & 5.09 & 4.58 & 4.15 & 5.75 & 4.83 & 4.93 & 5.17 & 4.44 & 4.49 & 5.26 & 4.36 & 5.03 & 4.98 & 4.88 & 3.87 & 4.14\end{array}$ $\begin{array}{lllllllllllllllllllll}5.65 & 4.86 & 5.19 & 6.14 & 7.16 & 5.19 & 7.52 & 5.98 & 5.57 & 5.25 & 6.53 & 6.73 & 5.98 & 6.25 & 7.44 & 6.60 & 6.52\end{array}$ $\begin{array}{llllllllllllllllllll}7.39 & 5.86 & 8.46 & 6.49 & 7.20 & 6.04 & 7.65 & 7.00 & 7.70 & 7.94 & 6.60 & 6.05 & 6.76 & 5.35 & 6.46 & 8.09 & 6.96\end{array}$ $\begin{array}{lllllllllllllllllll}6.79 & 4.32 & 5.98 & 6.73 & 5.46 & 6.50 & 5.50 & 4.50 & 6.05 & 5.01 & 5.92 & 5.86 & 4.20 & 5.00 & 5.75 & 5.11 & 5.49\end{array}$ $\begin{array}{llllllllllllllllllllll}5.17 & 5.51 & 5.36 & 4.94 & 5.15 & 3.65 & 5.33 & 6.09 & 3.47 & 2.91 & 5.52 & 5.14 & 3.98 & 4.15 & 5.27 & 4.87 & 4.61\end{array}$ $\begin{array}{llllllllllllllllllll}5.06 & 4.62 & 3.78 & 3.88 & 4.09 & 4.71 & 3.39 & 3.44 & 4.51 & 3.94 & 4.95 & 4.00 & 3.73 & 5.05 & 3.72 & 4.66 & 3.62\end{array}$ $\begin{array}{llllllllllllllllllll}4.57 & 3.08 & 4.93 & 4.02 & 3.03 & 4.39 & 3.61 & 3.33 & 3.78 & 4.81 & 4.59 & 4.04 & 4.83 & 5.12 & 3.80 & 3.80 & 4.32\end{array}$ $\begin{array}{llllllllllllllllllllll}4.07 & 3.85 & 3.76 & 3.58 & 3.95 & 5.12 & 3.64 & 3.08 & 3.82 & 3.44 & 3.32 & 3.69 & 2.86 & 3.56 & 3.95 & 3.34 & 4.60\end{array}$ $\begin{array}{lllllllllllllllllllll}3.62 & 2.60 & 4.16 & 4.15 & 3.18 & 2.56 & 3.63 & 3.59 & 4.29 & 4.26 & 4.86 & 3.88 & 2.93 & 3.09 & 2.19 & 2.86 & 4.04\end{array}$ \begin{tabular}{ll|l|l|l|l|l|lllllllllllll}
3.86 & 2.35 & 2.90 & 3.66 & 3.91 & 2.98 & 4.32 & 3.83 & 4.40 & 3.32 & 3.25 & 4.05 & 4.03 & 2.49 & 2.89 & 2.05 & 3.17
\end{tabular} $\begin{array}{llllllllllllllllll}3.23 & 3.84 & 4.04 & 3.62 & 3.87 & 3.16 & 3.65 & 3.38 & 2.96 & 4.20 & 4.08 & 4.32 & 1.83 & 3.77 & 3.02 & 3.57 & 3.29\end{array}$ $\begin{array}{lllllllllllllllllllll}3.57 & 2.84 & 2.81 & 3.79 & 3.83 & 3.28 & 3.82 & 1.58 & 3.21 & 3.62 & 3.51 & 4.19 & 4.00 & 3.14 & 3.67 & 3.53 & 3.20\end{array}$ $\begin{array}{lllllllllllllllllll}4.12 & 3.87 & 4.00 & 3.43 & 1.98 & 4.37 & 3.10 & 3.05 & 4.72 & 3.52 & 3.35 & 2.50 & 4.16 & 3.12 & 3.51 & 3.68 & 2.83\end{array}$ $\begin{array}{llllllllllllllllllll}4.86 & 2.74 & 3.96 & 3.06 & 4.47 & 2.72 & 2.89 & 2.73 & 3.15 & 2.59 & 4.81 & 3.14 & 4.33 & 3.10 & 3.89 & 3.30 & 3.90\end{array}$ $\begin{array}{llllllllllllllllllllll}2.61 & 2.81 & 4.08 & 2.79 & 3.04 & 2.79 & 3.26 & 2.41 & 3.05 & 3.51 & 2.79 & 3.78 & 2.71 & 3.86 & 3.75 & 4.44 & 3.31\end{array}$ $\begin{array}{lllllllllllllllllllllll}3.19 & 4.26 & 4.19 & 3.84 & 3.29 & 3.93 & 4.47 & 3.49 & 3.33 & 4.06 & 4.45 & 3.72 & 4.33 & 3.06 & 2.63 & 2.77 & 3.10\end{array}$ $\begin{array}{lllllllllllllllllllll}3.30 & 2.92 & 4.40 & 4.10 & 4.14 & 4.04 & 4.28 & 3.66 & 3.31 & 3.01 & 4.27 & 3.67 & 3.65 & 3.45 & 3.05 & 3.53 & 4.13\end{array}$ $\begin{array}{lllllllllllllllllllll}3.16 & 3.30 & 3.71 & 2.73 & 2.53 & 2.64 & 3.04 & 4.40 & 4.02 & 3.12 & 3.87 & 4.27 & 4.05 & 3.71 & 4.19 & 3.98 & 2.74\end{array}$ $\begin{array}{llllllllllllllllllllll}4.38 & 4.08 & 3.79 & 4.83 & 3.89 & 4.17 & 3.63 & 4.91 & 4.80 & 5.48 & 3.04 & 3.98 & 4.82 & 2.50 & 4.57 & 4.31 & 3.85\end{array}$ $\begin{array}{lllllllllllllllllllll}5.06 & 4.72 & 4.42 & 5.10 & 6.00 & 4.97 & 5.73 & 4.35 & 5.17 & 3.77 & 4.21 & 4.64 & 4.35 & 3.82 & 4.35 & 3.34 & 4.49\end{array}$ $\begin{array}{lllllllllllllllllllll}5.89 & 5.19 & 5.22 & 4.37 & 3.39 & 4.63 & 5.59 & 6.87 & 6.49 & 4.87 & 6.60 & 4.75 & 5.35 & 5.13 & 5.26 & 4.62 & 3.91\end{array}$ $\begin{array}{llllllllllllllllllllllll}3.13 & 6.46 & 6.45 & 6.41 & 6.93 & 6.80 & 7.80 & 4.70 & 7.40 & 7.22 & 7.37 & 7.12 & 9.11 & 8.43 & 5.47 & 7.33 & 5.99\end{array}$

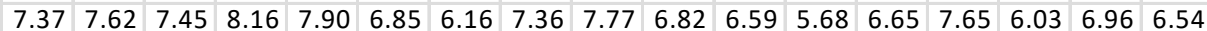
$\begin{array}{llllllllllllllllllll}6.13 & 6.57 & 5.44 & 6.00 & 5.76 & 4.92 & 4.99 & 4.79 & 3.81 & 4.95 & 4.97 & 3.96 & 4.44 & 3.43 & 5.47 & 6.00 & 5.22\end{array}$ $\begin{array}{lllllllllllllllllll}5.29 & 5.43 & 2.88 & 5.07 & 5.07 & 3.32 & 3.89 & 4.02 & 4.24 & 2.73 & 3.25 & 2.95 & 2.72 & 3.29 & 4.45 & 3.37 & 4.91\end{array}$ $\begin{array}{lllllllllllllllllllllll}2.31 & 3.26 & 2.45 & 3.14 & 3.50 & 2.40 & 2.53 & 3.13 & 2.27 & 1.94 & 2.89 & 1.63 & 2.52 & 1.92 & 2.11 & 2.62 & 2.54\end{array}$ $\begin{array}{llllllllllllllllllllllll}2.32 & 1.58 & 2.35 & 1.74 & 1.63 & 0.00 & 1.98 & 2.05 & 0.00 & 1.27 & 0.00 & 0.00 & 0.00 & 0.00 & 1.45 & 0.00 & 1.20\end{array}$ $\begin{array}{lllllllllllllllllll}0.00 & 0.00 & 0.00 & 0.00 & 0.00 & 0.00 & 0.00 & 0.00 & 0.00 & 0.00 & 0.00 & 0.00 & 0.00 & 0.00 & 0.00 & 0.00 & 0.00\end{array}$ $\begin{array}{lllllllllllllllllllll}0.00 & 0.00 & 0.00 & 0.00 & 0.00 & 0.00 & 0.00 & 0.00 & 0.00 & 0.00 & 0.00 & 0.00 & 0.00 & 0.00 & 0.00 & 0.00 & 0.00\end{array}$

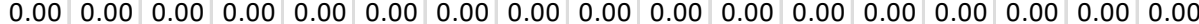
$\begin{array}{llllllllllllllllllll}0.00 & 0.00 & 0.00 & 0.00 & 0.00 & 0.00 & 0.00 & 0.00 & 0.00 & 0.00 & 0.00 & 0.00 & 0.00 & 0.00 & 0.00 & 0.00 & 0.00\end{array}$ 


\section{Cs-134 Input R matrix}

\begin{tabular}{|l|l|l|l|l|l|l|l|l|l|l|l|l|l|l|l|l|}
\hline 0.00 & 0.00 & 0.00 & 0.00 & 0.00 & 0.00 & 0.00 & 0.00 & 0.00 & 0.00 & 0.00 & 0.00 & 0.00 & 0.00 & 0.00 & 0.00 & 0.00 \\
\hline
\end{tabular} \begin{tabular}{|l|l|l|l|l|l|l|l|l|l|l|l|l|l|l|l|l|}
\hline 0.00 & 0.00 & 0.00 & 0.00 & 0.00 & 0.00 & 0.00 & 0.00 & 0.00 & 0.00 & 0.00 & 0.00 & 0.00 & 0.00 & 0.00 & 0.64 & 0.00 \\
\hline
\end{tabular}

\begin{tabular}{|l|l|l|l|l|l|l|l|l|l|l|l|l|l|l|l|l|}
\hline 0.00 & 0.00 & 0.00 & 0.00 & 0.00 & 0.00 & 0.00 & 0.00 & 0.00 & 0.00 & 0.00 & 0.00 & 0.00 & 0.00 & 0.00 & 0.00 & 0.00 \\
\hline
\end{tabular} \begin{tabular}{|l|l|l|l|l|l|l|l|l|l|l|l|l|l|l|l|l|l|l|l|l|l|}
\hline 0.00 & 0.79 & 0.00 & 0.00 & 0.69 & 1.30 & 0.00 & 0.81 & 0.00 & 0.94 & 1.00 & 1.63 & 1.34 & 1.05 & 0.78 & 0.69 & 1.10 \\
\hline
\end{tabular} \begin{tabular}{|l|l|l|l|l|l|l|l|l|l|l|l|l|l|l|l|l|}
\hline 2.18 & 1.60 & 1.78 & 2.62 & 1.92 & 2.99 & 2.00 & 3.25 & 2.11 & 3.19 & 2.34 & 3.08 & 3.78 & 3.33 & 2.38 & 2.30 & 2.37 \\
\hline
\end{tabular} \begin{tabular}{|l|l|l|l|l|l|l|l|l|l|l|l|l|l|l|l|l|l|l|l|l|}
3.47 & 3.67 & 4.64 & 3.05 & 4.38 & 4.86 & 4.72 & 3.73 & 3.12 & 5.59 & 4.51 & 4.72 & 4.41 & 5.26 & 4.38 & 3.51 & 4.11 \\
\hline
\end{tabular} \begin{tabular}{|l|l|l|l|l|l|l|l|l|l|l|l|l|l|l|l|l|}
\hline 4.90 & 5.04 & 6.09 & 5.36 & 5.35 & 6.67 & 5.88 & 6.14 & 6.03 & 5.69 & 5.25 & 7.28 & 6.03 & 6.71 & 5.75 & 6.53 & 6.74 \\
\hline
\end{tabular} \begin{tabular}{|l|l|l|l|l|l|l|l|l|l|l|l|l|l|l|l|l|l|}
\hline 8.39 & 7.77 & 6.40 & 7.18 & 9.91 & 9.00 & 8.06 & 8.41 & 8.23 & 7.80 & 8.00 & 7.97 & 7.89 & 7.76 & 8.86 & 8.40 & 6.14 \\
\hline
\end{tabular}

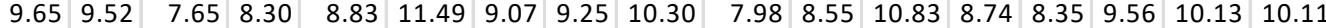
\begin{tabular}{|l|l|l|l|l|l|l|l|l|l|l|l|l|l|l|l|l|l|l|}
\hline 8.95 & 9.41 & 7.47 & 8.86 & 6.60 & 6.52 & 5.80 & 6.42 & 7.67 & 5.97 & 7.18 & 6.65 & 7.94 & 7.92 & 6.64 & 7.42 & 7.92 \\
\hline
\end{tabular}

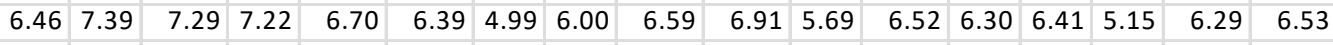

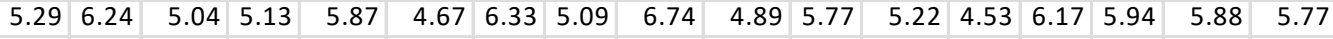
\begin{tabular}{|l|l|l|l|l|l|l|l|l|l|l|l|l|l|l|l|l|}
7.46 & 4.54 & 5.29 & 5.62 & 6.02 & 4.50 & 4.86 & 6.06 & 5.49 & 5.37 & 5.16 & 6.36 & 3.93 & 4.82 & 4.58 & 6.26 & 5.66 \\
\hline
\end{tabular}

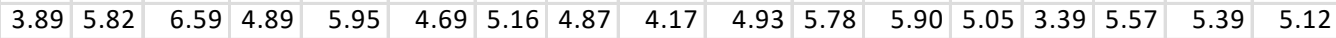
\begin{tabular}{|l|l|l|l|l|l|l|l|l|l|l|l|l|l|l|l|l|l|l|l|}
\hline 4.79 & 5.64 & 5.45 & 5.26 & 5.07 & 5.22 & 4.41 & 5.13 & 5.05 & 4.69 & 4.20 & 4.81 & 4.74 & 6.12 & 5.55 & 4.02 & 6.92 \\
\hline
\end{tabular} \begin{tabular}{|l|l|l|l|l|l|l|l|l|l|l|l|l|l|l|l|l|}
\hline 4.87 & 4.50 & 5.71 & 3.91 & 5.59 & 4.52 & 4.38 & 5.52 & 4.80 & 6.65 & 5.22 & 4.82 & 3.62 & 4.13 & 5.14 & 4.09 & 5.74 \\
\hline
\end{tabular} \begin{tabular}{|l|l|l|l|l|l|l|l|l|l|l|l|l|l|l|l|l|l|l|l|}
\hline 4.57 & 3.80 & 5.47 & 4.62 & 4.11 & 4.33 & 5.43 & 4.61 & 5.27 & 4.92 & 4.88 & 5.20 & 5.42 & 5.42 & 4.35 & 3.90 & 4.05 \\
\hline
\end{tabular} \begin{tabular}{|l|l|l|l|l|l|l|l|l|l|l|l|l|l|l|l|l|l|}
\hline 4.64 & 4.41 & 4.72 & 5.00 & 4.52 & 5.17 & 4.84 & 5.29 & 5.29 & 4.47 & 4.37 & 5.09 & 3.22 & 4.18 & 3.85 & 5.05 & 4.22 \\
\hline
\end{tabular} \begin{tabular}{|l|l|l|l|l|l|l|l|l|l|l|l|l|l|l|l|l|}
\hline 5.27 & 4.66 & 4.87 & 4.13 & 4.65 & 5.09 & 3.91 & 4.29 & 3.55 & 5.23 & 5.41 & 4.53 & 4.00 & 4.04 & 4.35 & 3.29 & 3.75 \\
\hline
\end{tabular} \begin{tabular}{|l|l|l|l|l|l|l|l|l|l|l|l|l|l|l|l|l|l|l|l|l|}
4.67 & 4.34 & 5.95 & 4.24 & 5.28 & 4.82 & 4.76 & 4.67 & 5.66 & 4.88 & 5.54 & 5.42 & 5.17 & 5.13 & 4.56 & 4.44 & 4.24 \\
\hline
\end{tabular} \begin{tabular}{|l|l|l|l|l|l|l|l|l|l|l|l|l|l|l|l|l|l|l|l|}
\hline 4.83 & 6.13 & 5.03 & 4.93 & 4.46 & 4.92 & 4.55 & 6.87 & 4.22 & 4.09 & 4.95 & 5.44 & 5.41 & 5.27 & 4.89 & 4.62 & 4.78 \\
\hline
\end{tabular} \begin{tabular}{|l|l|l|l|l|l|l|l|l|l|l|l|l|l|l|l|l|l|l|}
\hline 5.50 & 4.37 & 5.14 & 5.21 & 5.49 & 4.31 & 5.62 & 4.60 & 4.51 & 5.58 & 5.12 & 4.71 & 4.78 & 4.81 & 4.51 & 5.69 & 6.06 \\
\hline 4.26 & 4.39 & 4.78 & 5.86 & 6.11 & 5.47 & 5.21 & 5.79 & 4.67 & 4.91 & 4.07 & 5.30 & 6.10 & 4.81 & 4.84 & 5.43 & 6.25 \\
\hline
\end{tabular} \begin{tabular}{|l|l|l|l|l|l|l|l|l|l|l|l|l|l|l|l|l|}
\hline 4.26 & 4.39 & 4.78 & 5.86 & 6.11 & 5.47 & 5.21 & 5.79 & 4.67 & 4.91 & 4.07 & 5.30 & 6.10 & 4.81 & 4.84 & 5.43 & 6.25 \\
\hline
\end{tabular}

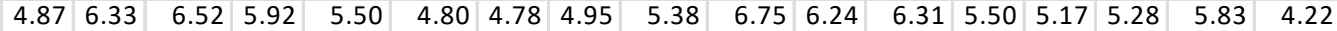
\begin{tabular}{|l|l|l|l|l|l|l|l|l|l|l|l|l|l|l|l|l|l|}
\hline 5.69 & 5.15 & 5.64 & 7.45 & 6.58 & 5.42 & 5.59 & 5.43 & 5.48 & 5.34 & 6.66 & 6.66 & 5.94 & 5.32 & 6.10 & 4.76 & 4.44 \\
\hline
\end{tabular} \begin{tabular}{|l|l|l|l|l|l|l|l|l|l|l|l|l|l|l|l|l|l|l|}
\hline 6.14 & 6.07 & 7.56 & 6.96 & 5.89 & 5.18 & 6.43 & 6.17 & 6.83 & 6.63 & 6.32 & 7.09 & 6.18 & 6.01 & 6.07 & 6.13 & 7.04 \\
\hline 8.96 & 7.44 & 6.79 & 7.07 & 7.01 & 8.06 & 6.38 & 7.09 & 8.00 & 7.83 & 8.20 & 7.83 & 7.88 & 7.48 & 6.86 & 8.41 & 9.01 \\
\hline
\end{tabular} \begin{tabular}{|l|l|l|l|l|l|l|l|l|l|l|l|l|l|l|l|l|l|}
8.96 & 7.44 & 6.79 & 7.07 & 7.01 & 8.06 & 6.38 & 7.09 & 8.00 & 7.83 & 8.20 & 7.83 & 7.88 & 7.48 & 6.86 & 8.41 & 9.01 \\
\hline
\end{tabular}

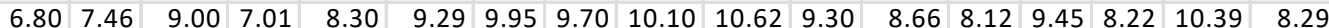
\begin{tabular}{|l|l|l|l|l|l|l|l|l|l|l|l|l|l|l|l|l|l|l|l|}
\hline 8.50 & 9.79 & 11.22 & 9.11 & 11.38 & 10.52 & 8.64 & 9.66 & 9.98 & 8.68 & 9.14 & 8.08 & 7.13 & 8.97 & 8.98 & 7.20 & 10.17 \\
\hline 9.21 & 6.53 & 7.02 & 7.39 & 7.98 & 7.79 & 6.06 & 6.82 & 6.26 & 6.30 & 6.84 & 5.81 & 5.51 & 6.61 & 7.22 & 7.38 & 6.72 \\
\hline
\end{tabular}

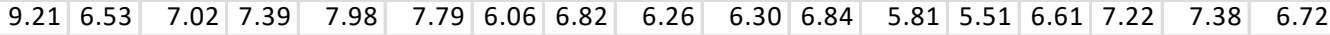
\begin{tabular}{|l|l|l|l|l|l|l|l|l|l|l|l|l|l|l|l|l|}
5.49 & 5.75 & 6.80 & 4.82 & 6.06 & 5.73 & 5.79 & 5.36 & 5.32 & 5.27 & 4.03 & 4.01 & 4.49 & 3.75 & 4.72 & 4.78 & 5.19 \\
\hline
\end{tabular} \begin{tabular}{|l|l|l|l|l|l|l|l|l|l|l|l|l|l|l|l|l|}
\hline 4.21 & 3.59 & 5.62 & 3.38 & 3.58 & 3.22 & 3.01 & 3.52 & 2.94 & 3.11 & 3.50 & 3.05 & 3.12 & 2.44 & 3.53 & 2.70 & 4.27 \\
\hline
\end{tabular} \begin{tabular}{|l|l|l|l|l|l|l|l|l|l|l|l|l|l|l|l|l|}
\hline 2.56 & 2.26 & 2.32 & 2.16 & 1.69 & 1.25 & 1.97 & 2.11 & 1.12 & 1.36 & 1.21 & 0.92 & 0.00 & 1.05 & 2.06 & 1.47 & 2.31 \\
\hline
\end{tabular} \begin{tabular}{|l|l|l|l|l|l|l|l|l|l|l|l|l|l|l|l|l}
0.00 & 0.00 & 0.00 & 0.00 & 0.00 & 0.00 & 0.00 & 0.00 & 0.00 & 0.00 & 0.00 & 0.00 & 0.00 & 0.00 & 0.00 & 0.00 & 0.00 \\
\hline
\end{tabular} \begin{tabular}{|l|l|l|l|l|l|l|l|l|l|l|l|l|l|l|l|l|}
\hline 0.00 & 0.00 & 0.67 & 0.00 & 0.00 & 0.00 & 0.00 & 0.00 & 0.00 & 0.00 & 0.00 & 0.00 & 0.00 & 0.56 & 0.00 & 0.00 & 0.54 \\
\hline
\end{tabular} \begin{tabular}{|l|l|l|l|l|l|l|l|l|l|l|l|l|l|l|l|l|}
\hline 0.00 & 0.00 & 0.00 & 0.00 & 0.00 & 0.00 & 0.00 & 0.00 & 0.00 & 0.00 & 0.00 & 0.00 & 0.00 & 0.00 & 0.00 & 0.00 & 0.00 \\
\hline
\end{tabular}

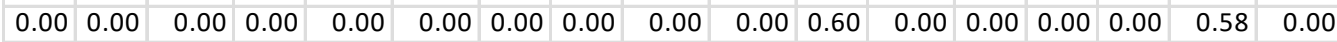




\section{Outer Ring 08 Axial Middle}

Angles for Middle OR-08 Scan

\section{$\begin{array}{llllllll}0.1 & 22.5 & 45 & 67.6 & 90 & 112.5 & 134.9 & 157.5\end{array}$}

\section{Cs-137 Input R matrix}

\begin{tabular}{|c|c|c|c|c|c|c|c|}
\hline & & & & & & & \\
\hline & & & & & & & \\
\hline .00 & & & & & & & \\
\hline 0.00 & & & & & & & \\
\hline 7.0 & & C & & & & & . \\
\hline 67 & 71 & 28 & & & & & .2 \\
\hline .25 & 78 & 35 & .80 & .61 & .49 & & 3.8 \\
\hline 6.39 & .70 & .14 & 8.07 & 3.49 & 2.41 & 7.14 & 9.9 \\
\hline .01 & .44 & 28 & L.16 & 2.04 & 9.28 & 4. & 3.1 \\
\hline .21 & 85 & .84 & 0.2 & & & & 1.3 \\
\hline 19 & .17 & .77 & .79 & & & & 9.9 \\
\hline 0.23 & .70 & .50 & 9.64 & 1.42 & 0.28 & 2.89 & 3.65 \\
\hline . 37 & & .52 & 7.29 & & & & .3 \\
\hline 96 & & & 4 & & & & 0.4 \\
\hline .72 & .25 & & & 39 & & & .1 \\
\hline 3.53 & .92 & .10 & 32.76 & 6.85 & 9.27 & 6.29 & 7.6 \\
\hline .96 & & & .47 & 13 & & 8. & 0.4 \\
\hline & & & & & & & .2 \\
\hline 8 & 2 & & 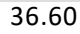 & & & & 3.4 \\
\hline .32 & .22 & .41 & 3.24 & 69 & 8.41 & & 0.8 \\
\hline 98 & & & 8 & & & & D. 3 \\
\hline 6 & & & & & & & 0.2 \\
\hline 5.28 & .31 & 3.59 & 2.9 & 5. & 1.21 & 6. & 8.6 \\
\hline 3.14 & .63 & 67 & 32.84 & 87 & 1.29 & 9.22 & 4.5 \\
\hline .41 & & & & & & & . \\
\hline 5.01 & & .73 & 33.89 & & & & 5.9 \\
\hline .38 & & .16 & 3 & & 2.49 & 5. & 7.2 \\
\hline .76 & & .87 & 46.4 & & & & 0.5 \\
\hline 2.82 & & & & & & & .1 \\
\hline 5.73 & & 7.68 & 64.62 & & 6.17 & 3.59 & 3.2 \\
\hline .66 & & .97 & 4 & U & 58.66 & 57.4 & 61.2 \\
\hline & & & & & & & \\
\hline & & & 37.39 & & .27 & .82 & 3.6 \\
\hline & & 89 & .94 & & .52 & 3.22 & 0.0 \\
\hline 17.0 & & 2.20 & 10 & & 19.17 & & 18.15 \\
\hline & & & & & & & .0 \\
\hline 0.00 & & & & & 00 & .00 & .0 \\
\hline $0 .($ & & & 0. & & 0.00 & 0.0 & $0.0 c$ \\
\hline 0.00 & 0.00 & 6.0 & 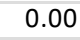 & & & 0.0 & 00 \\
\hline
\end{tabular}




\section{Cs-134 Input R matrix}

\begin{tabular}{|c|c|c|c|c|c|c|c|}
\hline & & & & & & & \\
\hline & & & & & & & \\
\hline .00 & & & & & & & \\
\hline .00 & & & & & & & \\
\hline 4 & 24 & & & & & & C \\
\hline 58 & & & & & & & . \\
\hline 99 & .99 & 85 & .27 & .05 & & & 3.3 \\
\hline .95 & .33 & .45 & .70 & .32 & & & 5.2 \\
\hline .15 & 57 & & 7 & 91 & & & 3.2 \\
\hline & & & & & & & \\
\hline .67 & .72 & 7 & 3 & 47 & & & 4.3 \\
\hline 5.32 & .77 & .22 & .66 & .37 & & 33 & 5.7 \\
\hline 02 & 59 & 16 & j. & .86 & & & 9.4 \\
\hline 92 & 2 & 89 & 5 & .10 & & & 5.4 \\
\hline .07 & .61 & .44 & 3.57 & 42 & .96 & & 7.1 \\
\hline .79 & .72 & .89 & 5.99 & 7.44 & & & 8.3 \\
\hline .25 & 47 & .66 & 38.65 & 5.58 & & & 9.0 \\
\hline 87 & & & 3 & & & & 4.2 \\
\hline & & & 2.31 & & .23 & & 5.1 \\
\hline .22 & .27 & .23 & 5.99 & 5.27 & 9 & 39 & 2.7 \\
\hline .07 & & & 3.78 & & & & 8.0 \\
\hline 98 & & & & & & & , \\
\hline 44 & & .79 & 8 & .25 & & & 8.1 \\
\hline .47 & & .48 & 05 & & & & 2.57 \\
\hline & & & & & & & 0.2 \\
\hline & & .93 & 3 & & & & 7.7 \\
\hline .24 & .28 & .24 & 3.57 & 98 & 9.54 & 48. & 7.0 \\
\hline 9.24 & & & & & 5.27 & & 4.32 \\
\hline .32 & & 19 & 52.05 & .88 & 0.77 & & 9.97 \\
\hline .37 & & .17 & 73.30 & 67.91 & 2.00 & 64. & 3.4 \\
\hline 72.57 & 2 & .84 & 61. & 67. & 5.96 & 73. & 7.7 \\
\hline & & & & & & & 4.10 \\
\hline 49.04 & & & 46.44 & & & 7.74 & 1.5. \\
\hline & & & .67 & .21 & .38 & 7.70 & 5.7 \\
\hline 17.88 & & & 10.59 & & 19.18 & 20.84 & $22.8 \varepsilon$ \\
\hline & & & & & & & .00 \\
\hline 0.00 & & & & & & 00 & .0 \\
\hline 0. & & & & & .00 & .00 & .06 \\
\hline & & & & & & & \\
\hline
\end{tabular}


Ag-110m Input R matrix

\begin{tabular}{|c|c|c|c|c|c|c|c|}
\hline 0.00 & 0.00 & 0.00 & 0.00 & 0.00 & 0.00 & 0.00 & \\
\hline 0.00 & 0.00 & 0.00 & 0.00 & 0.00 & 0.00 & 0.00 & 0.00 \\
\hline 0.00 & 0.00 & 0.00 & 0.00 & 0.00 & 0.00 & 0.00 & 0.00 \\
\hline 0.00 & 0.00 & 0.00 & 0.00 & 0.00 & 0.00 & 0.00 & 0.00 \\
\hline 0.00 & 0.00 & 0.00 & 0.00 & 0.00 & 0.00 & 0.00 & 0.00 \\
\hline 0.00 & 0.00 & 41.20 & 49.02 & 0.00 & 0.00 & 0.00 & 29.78 \\
\hline 41.17 & 56.56 & 64.00 & 0.00 & 81.45 & 48.50 & 58.33 & 53.71 \\
\hline 115.73 & 108.96 & 82.36 & 91.07 & 110.53 & 143.64 & 133.95 & 98.82 \\
\hline 174.15 & 158.21 & 134.34 & 160.16 & 167.12 & 184.17 & 138.69 & 105.39 \\
\hline 191.52 & 203.49 & 177.86 & 177.66 & 180.59 & 205.57 & 179.94 & 137.20 \\
\hline 137.39 & 153.27 & 132.90 & 152.36 & 167.97 & 169.20 & 159.77 & 117.94 \\
\hline 111.37 & 119.44 & 136.81 & 109.68 & 118.46 & 103.83 & 121.65 & 99.92 \\
\hline 85.16 & 95.37 & 110.53 & 125.42 & 116.32 & 82.49 & 81.58 & 77.93 \\
\hline 80.86 & 88.34 & 112.93 & 82.75 & 96.02 & 54.70 & 70.58 & 59.09 \\
\hline 73.90 & 88.93 & 102.33 & 97.71 & 78.97 & 64.42 & 47.51 & 73.06 \\
\hline 63.61 & 79.95 & 99.79 & 92.38 & 77.22 & 50.25 & 48.80 & 58.91 \\
\hline 55.35 & 75.07 & 93.61 & 72.86 & 64.98 & 51.37 & 45.33 & 53.23 \\
\hline 58.81 & 68.83 & 100.38 & 69.93 & 87.89 & 47.59 & 49.67 & 42.32 \\
\hline 59.66 & 66.48 & 80.93 & 78.65 & 77.28 & 45.13 & 32.61 & 64.43 \\
\hline 59.19 & 71.04 & 88.54 & 83.72 & 80.67 & 32.76 & 42.16 & 46.57 \\
\hline 53.09 & 60.72 & 78.78 & 67.79 & 56.73 & 58.09 & 47.42 & 43.24 \\
\hline 52.54 & 59.14 & 105.78 & 73.77 & 67.59 & 61.96 & 51.96 & 39.91 \\
\hline 47.64 & 52.67 & 58.78 & 72.47 & 51.53 & 37.94 & 35.82 & 39.26 \\
\hline 57.29 & 46.04 & 47.57 & 58.81 & 44.39 & 56.52 & 26.03 & 46.84 \\
\hline 60.68 & 53.15 & 40.65 & 51.96 & 51.48 & 63.11 & 51.37 & 63.78 \\
\hline 52.86 & 71.69 & 51.16 & 46.66 & 74.42 & 58.99 & 62.00 & 51.26 \\
\hline 58.22 & 44.21 & 44.56 & 34.19 & 60.94 & 62.26 & 48.21 & 62.57 \\
\hline 62.56 & 60.07 & 54.79 & 36.09 & 48.26 & 70.06 & 70.71 & 75.33 \\
\hline 68.57 & 56.35 & 46.78 & 48.87 & 67.66 & 69.74 & 89.64 & 105.52 \\
\hline 107.40 & 57.14 & 44.14 & 54.46 & 60.92 & 73.90 & 124.58 & 144.03 \\
\hline 94.20 & 57.00 & 32.16 & 0.00 & 55.45 & 94.33 & 123.21 & 183.58 \\
\hline 82.23 & 43.98 & 0.00 & 26.34 & 59.11 & 69.35 & 128.48 & 169.79 \\
\hline 33.13 & 29.87 & 19.89 & 0.00 & 45.31 & 60.53 & 66.48 & 87.04 \\
\hline 33.57 & 0.00 & 0.00 & 22.77 & 0.00 & 41.54 & 31.47 & 0.00 \\
\hline 0.00 & 0.00 & 0.00 & 0.00 & 0.00 & 21.60 & 0.00 & 0.00 \\
\hline 0.00 & 0.00 & 0.00 & 0.00 & 0.00 & 0.00 & 0.00 & 0.00 \\
\hline 0.00 & 0.00 & 0.00 & 0.00 & 0.00 & 0.00 & 0.00 & 0.00 \\
\hline 0.00 & 0.00 & 0.00 & 0.00 & 0.00 & 0.00 & 0.00 & 0.00 \\
\hline 0.00 & 0.00 & 0.00 & 0.00 & 0.00 & 0.00 & 0.00 & 0.00 \\
\hline
\end{tabular}




\section{Appendix E Gamma Tomograms}

\section{Inner Ring 3 Axial Center}

Cs-137

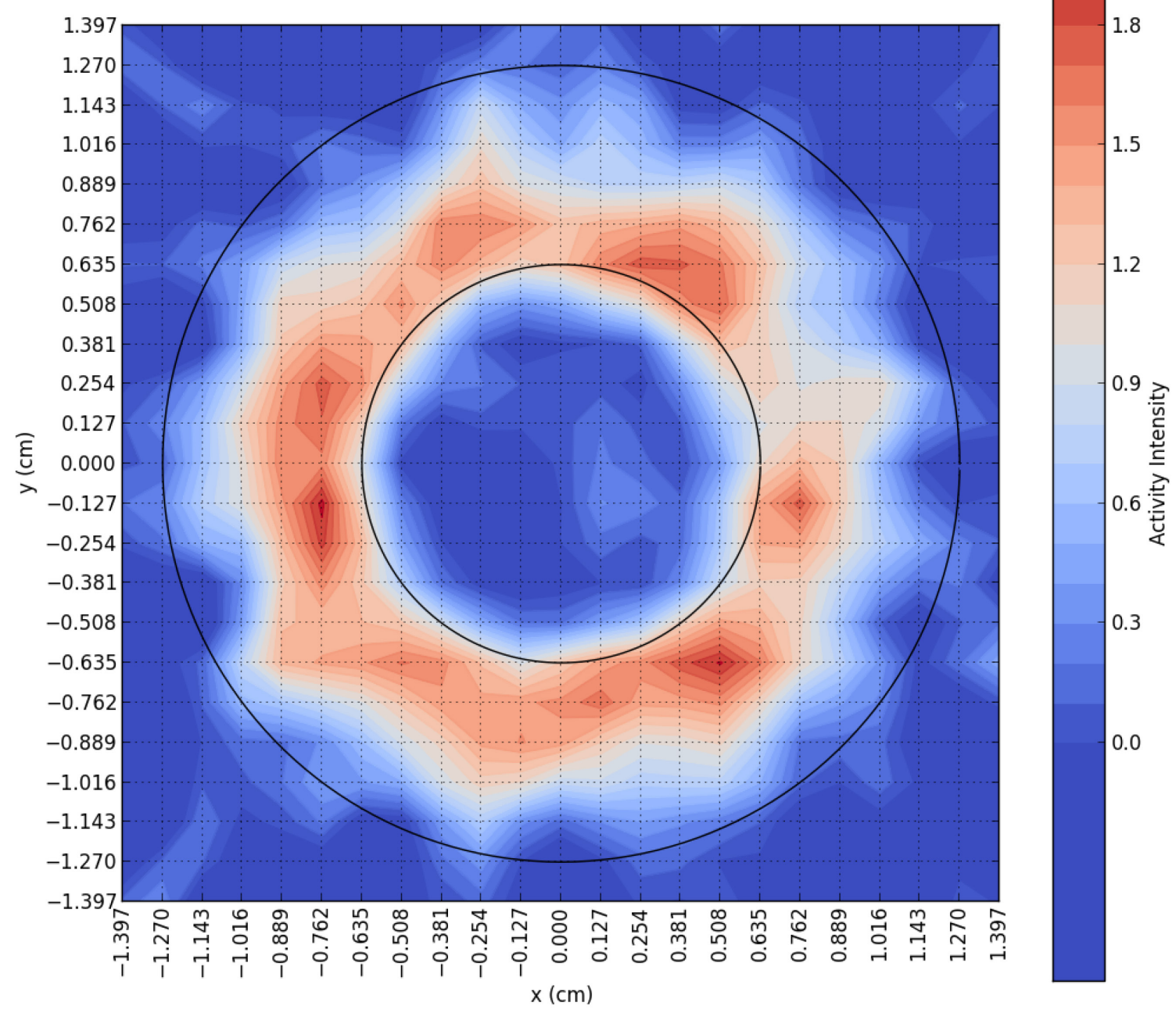


Cs-134

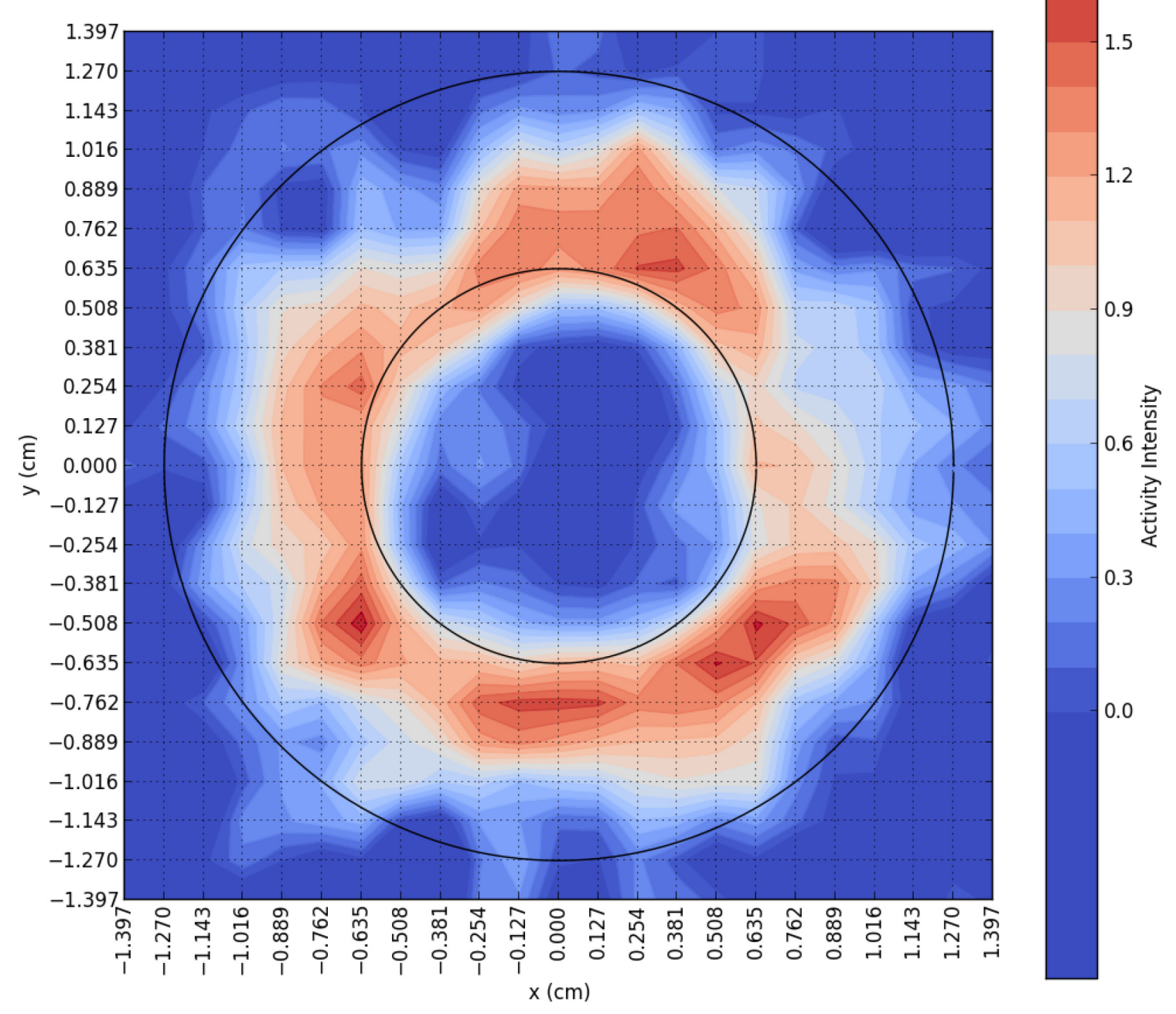


Co-60

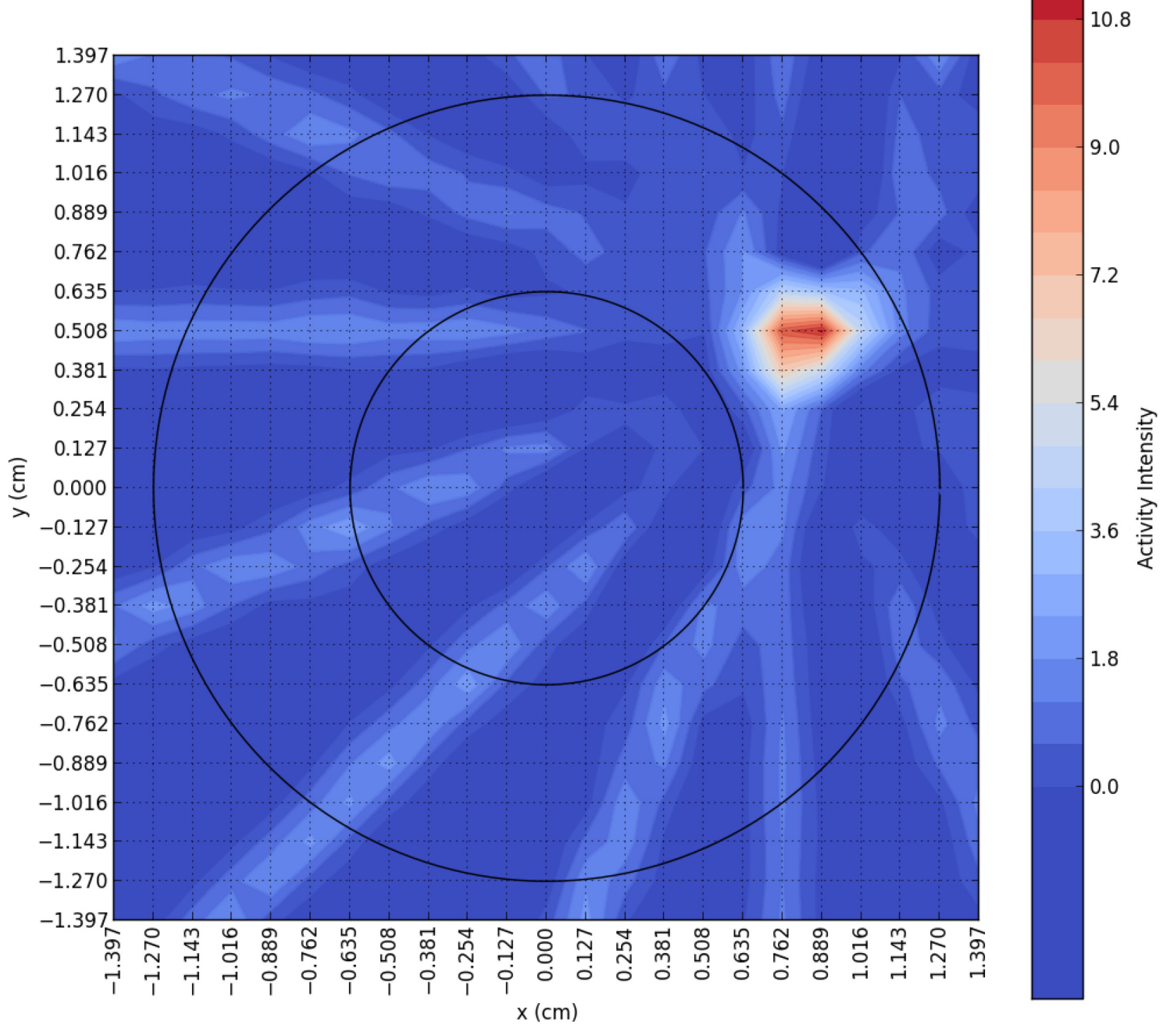




\section{Inner Ring 3 Axial Top}

Cs- 137

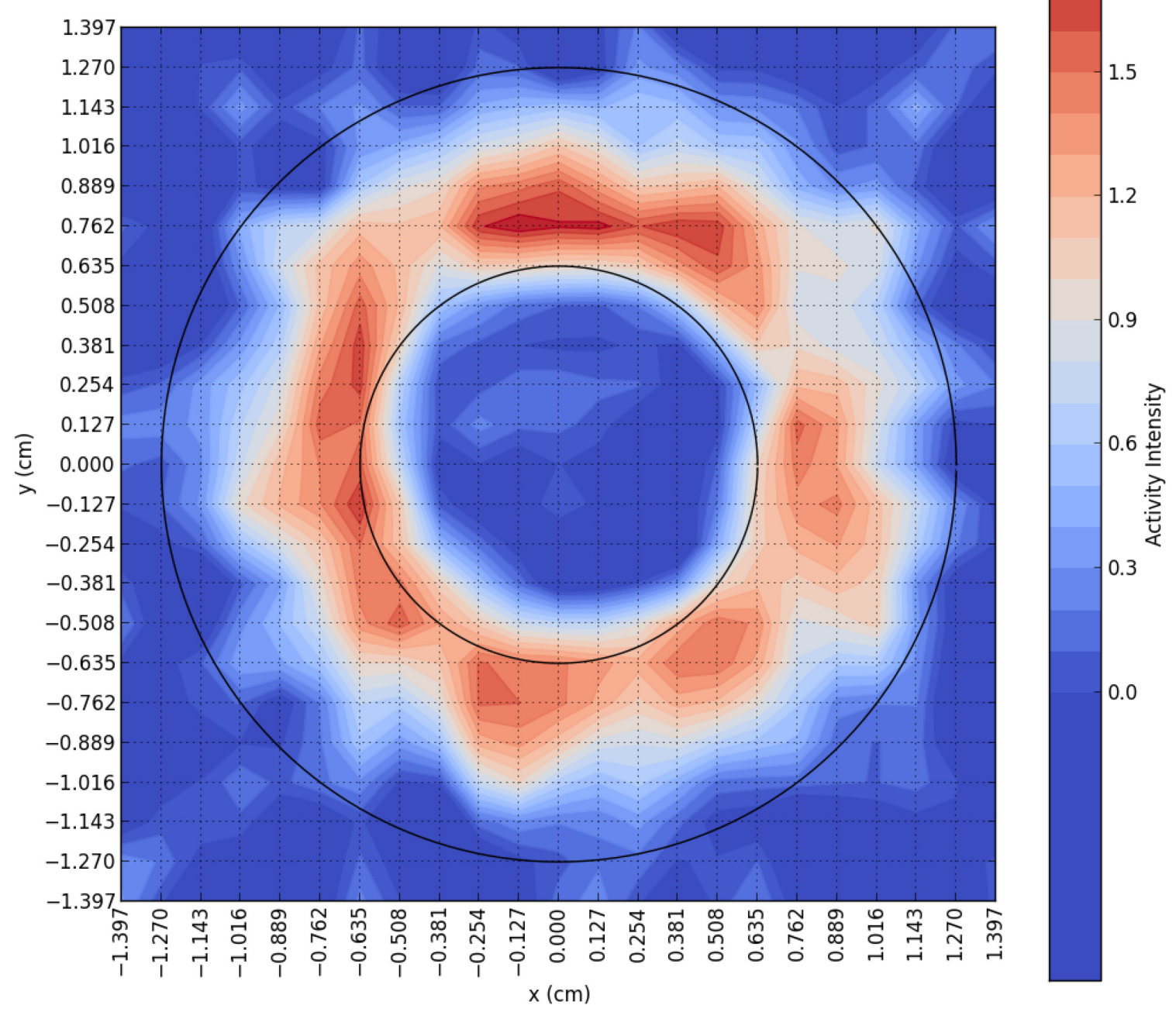


Cs-134

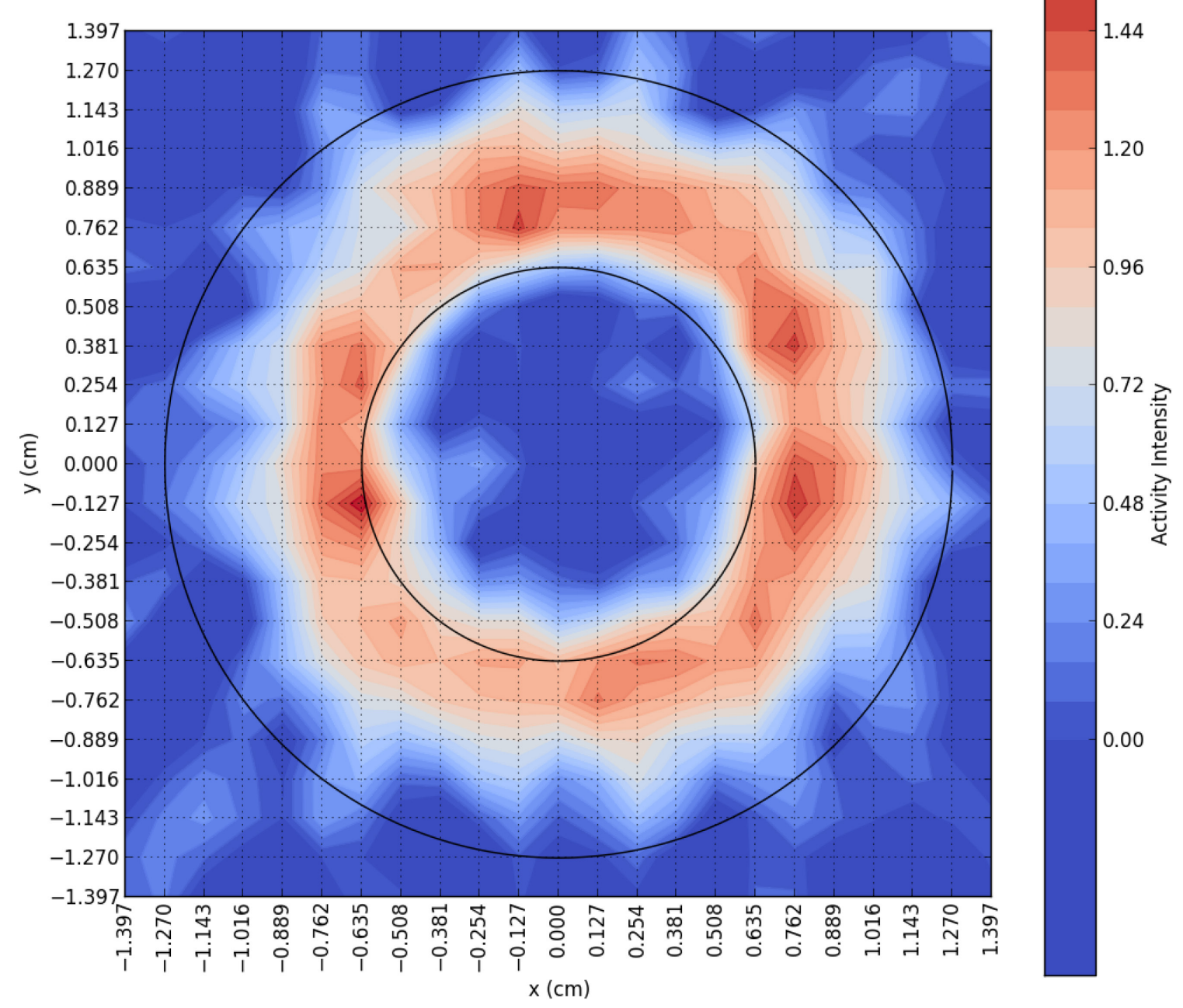


Co-60

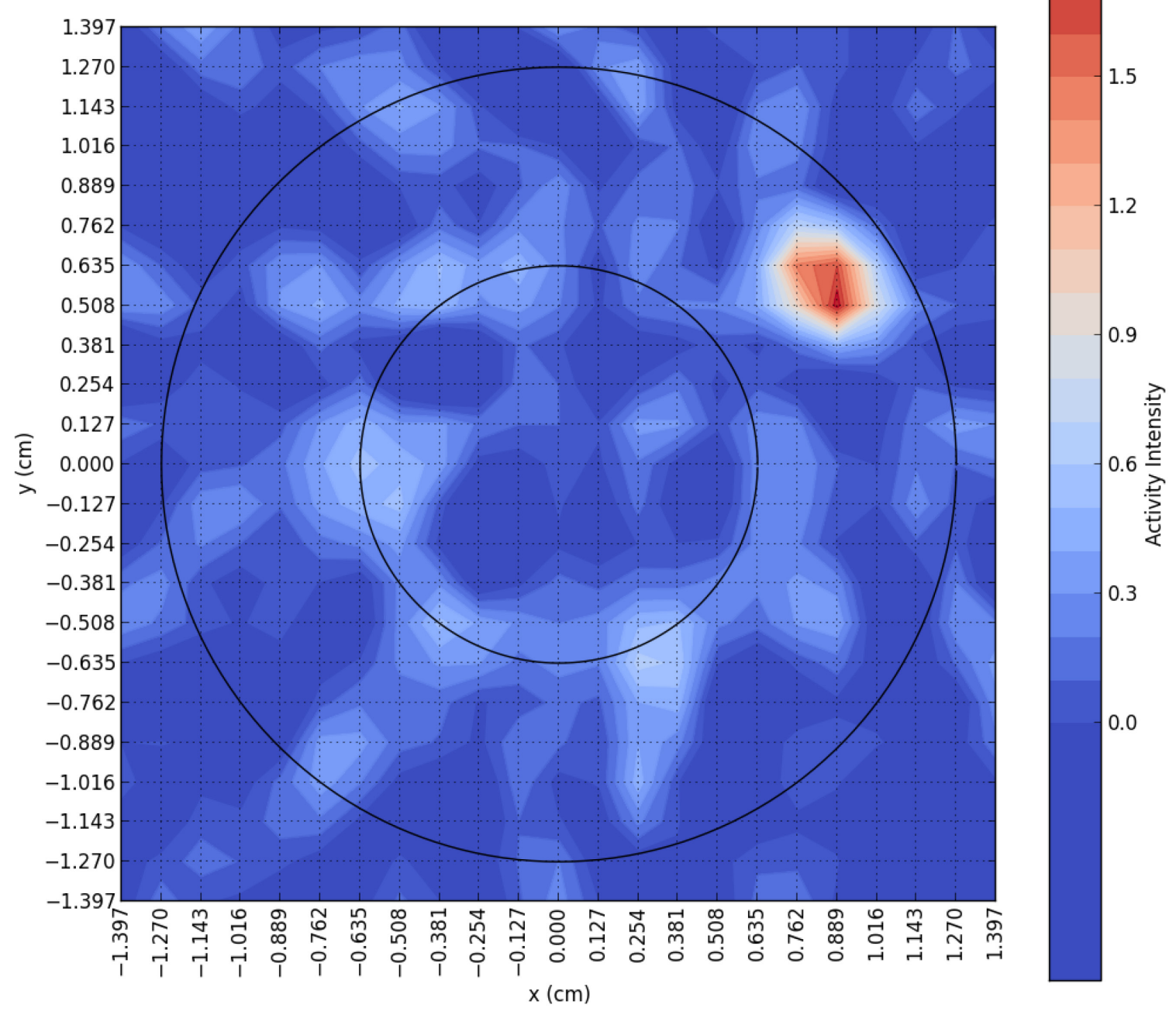


Ag-110m

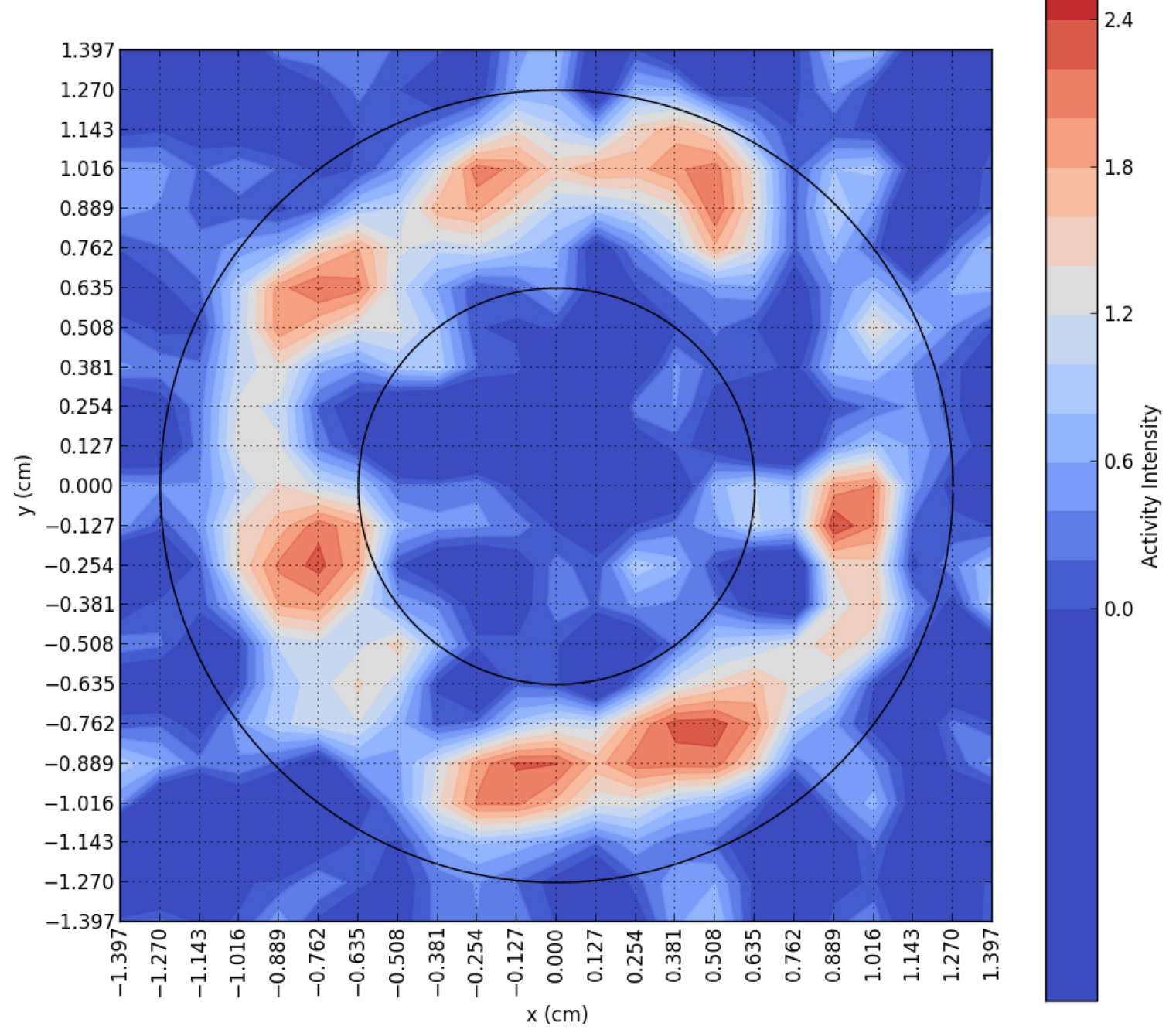




\section{Inner Ring 4 Axial Middle}

Cs-137

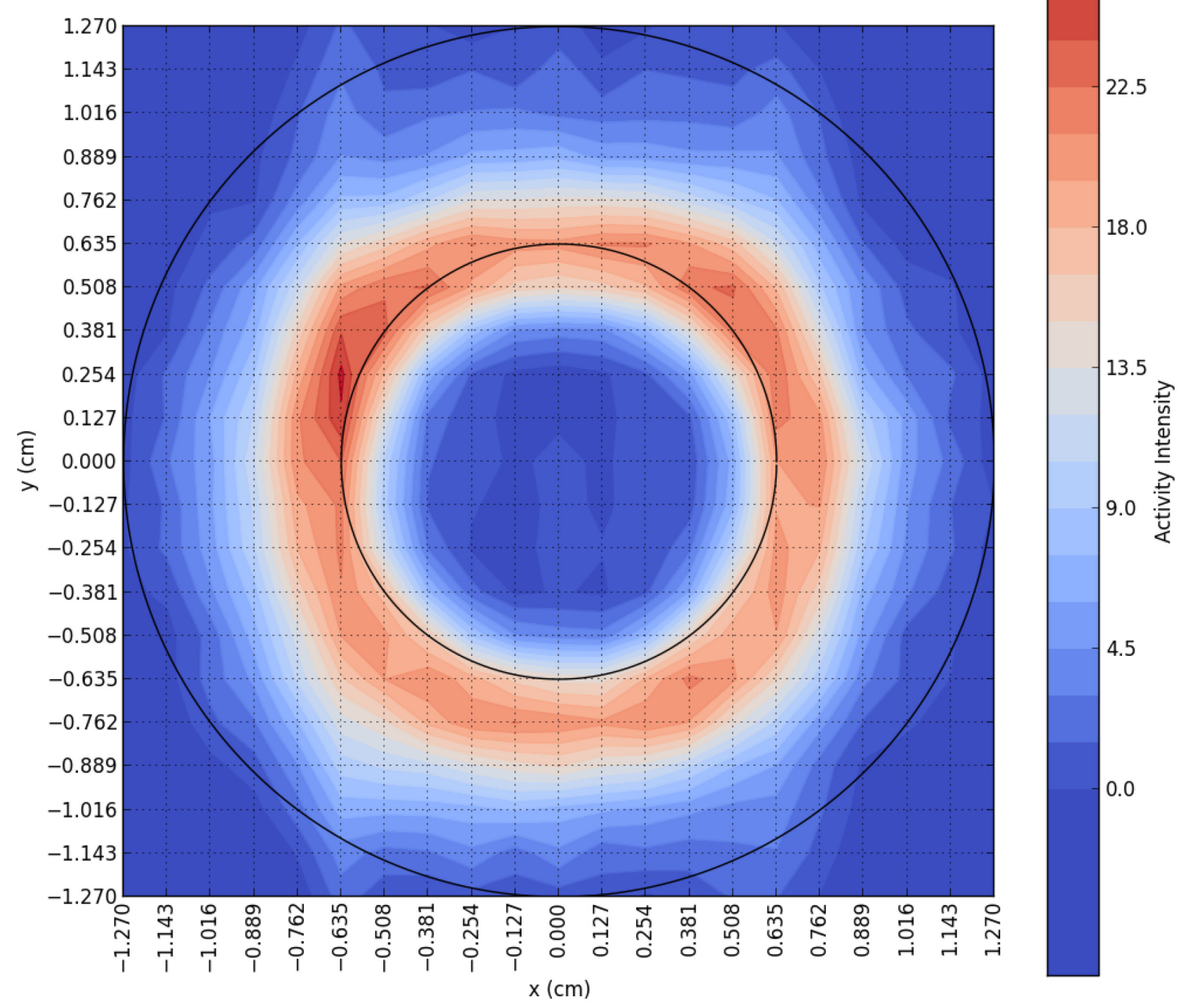


Cs-134

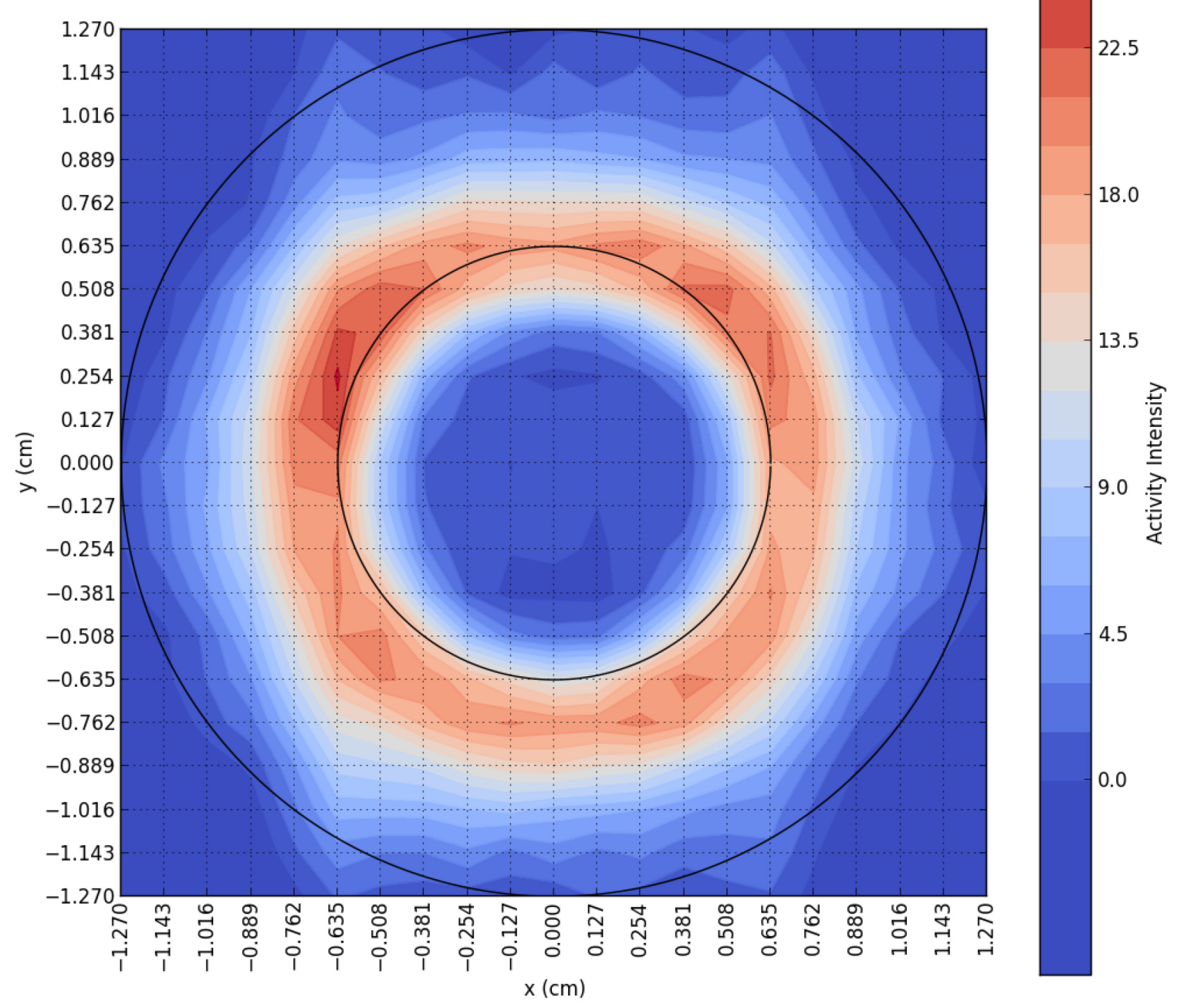




\section{Inner Ring 5 Axial Middle}

Cs-137

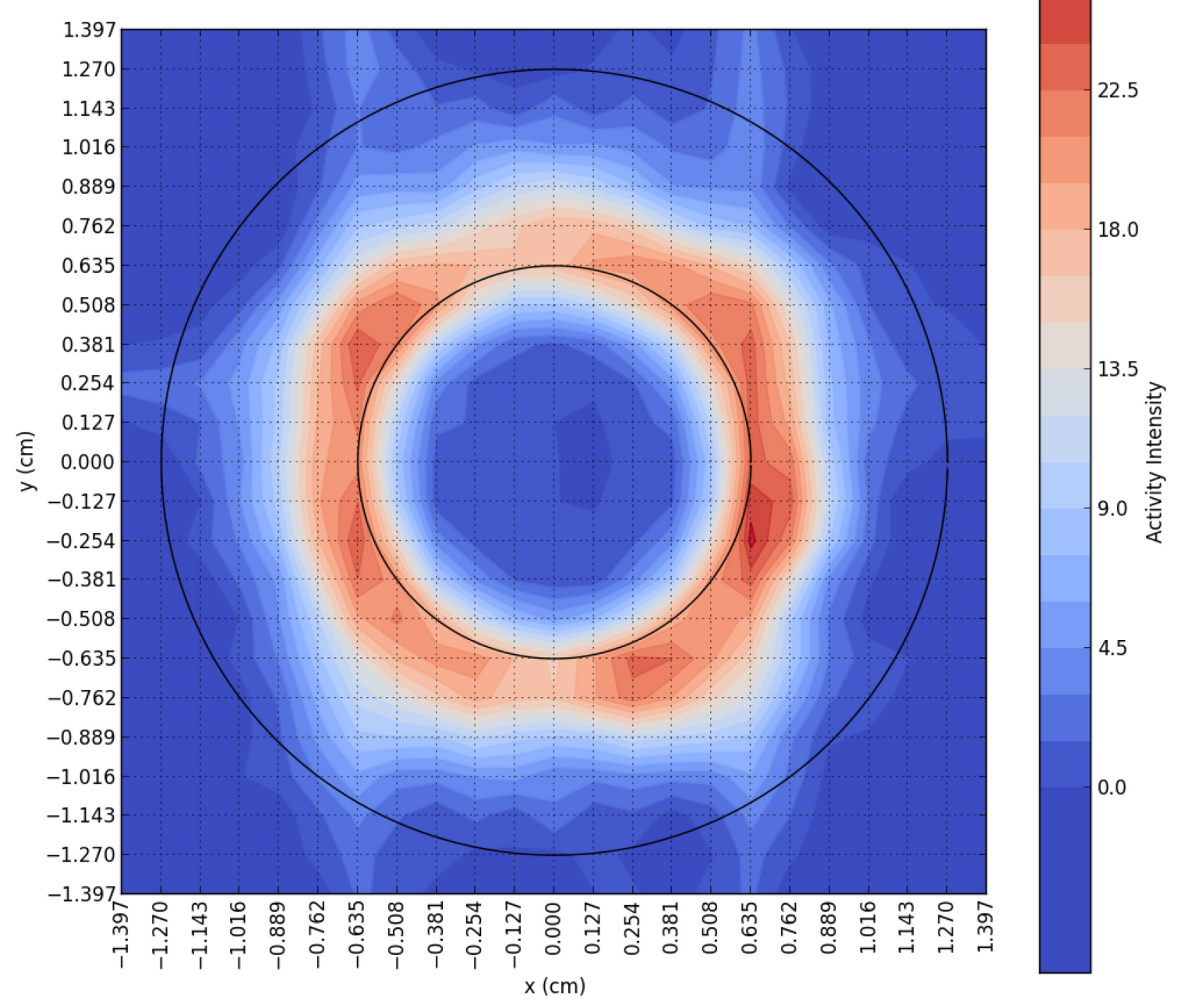


Cs-134

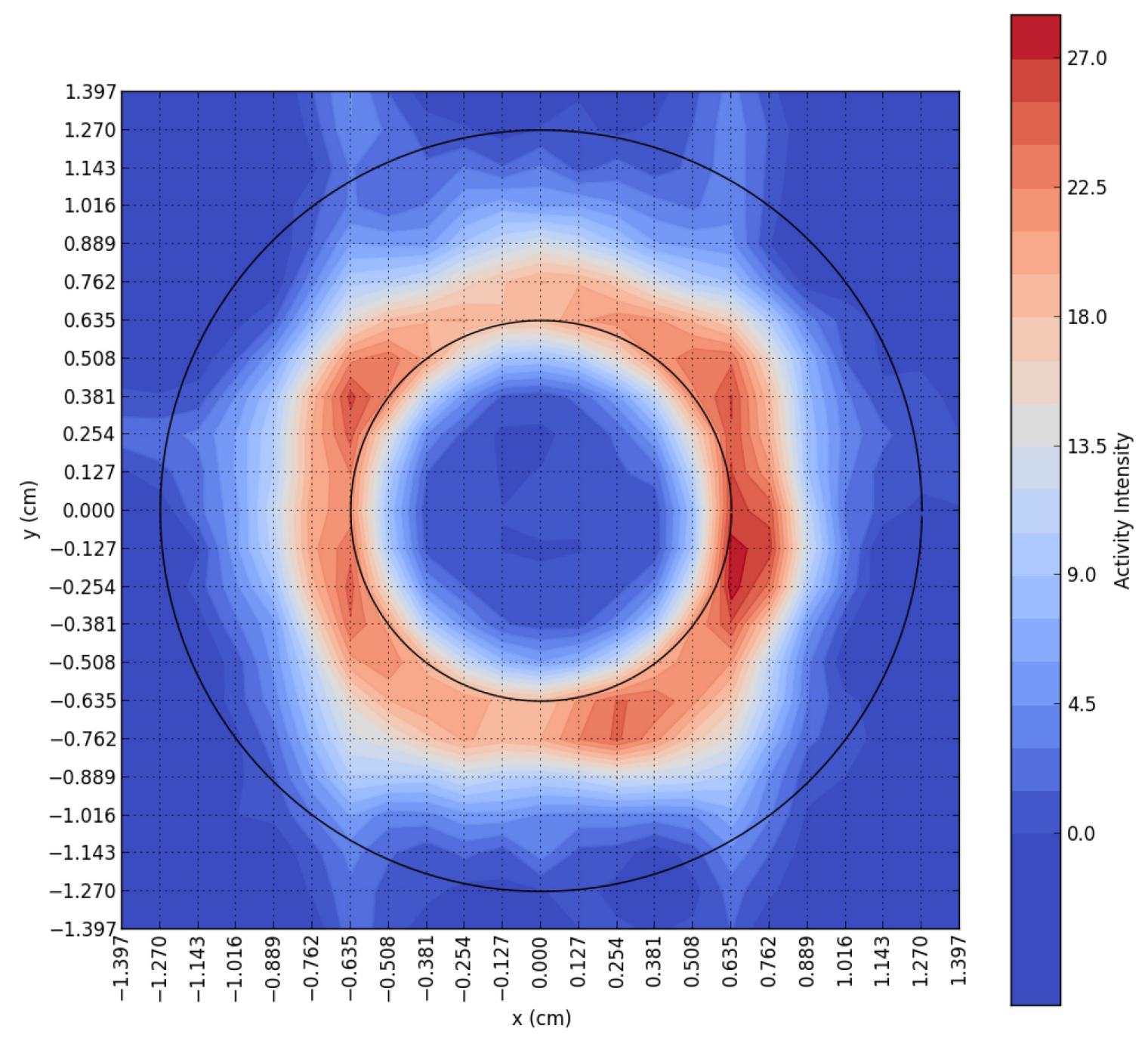




\section{Inner Ring 7 Axial Middle}

Cs-137

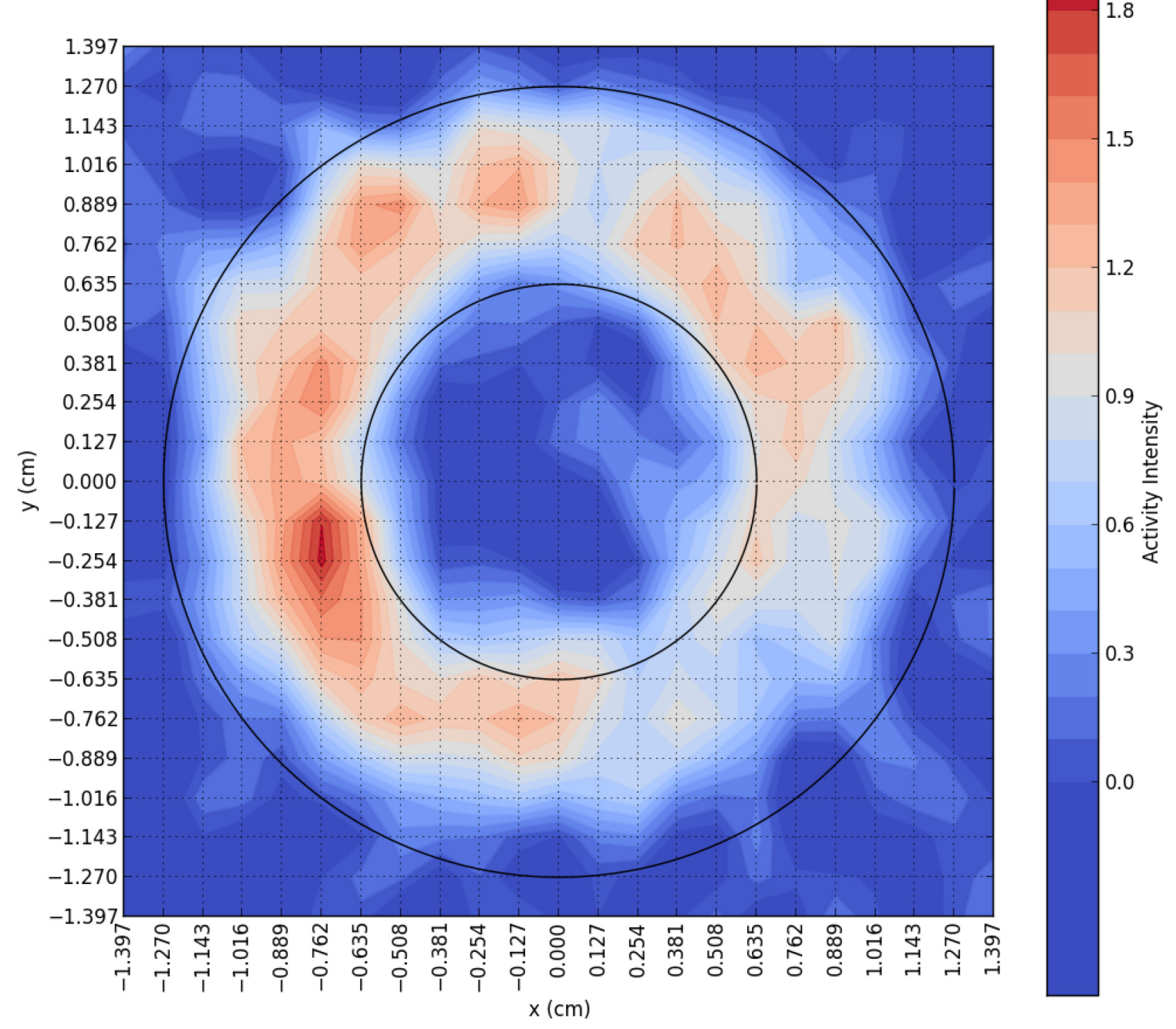


Cs-134

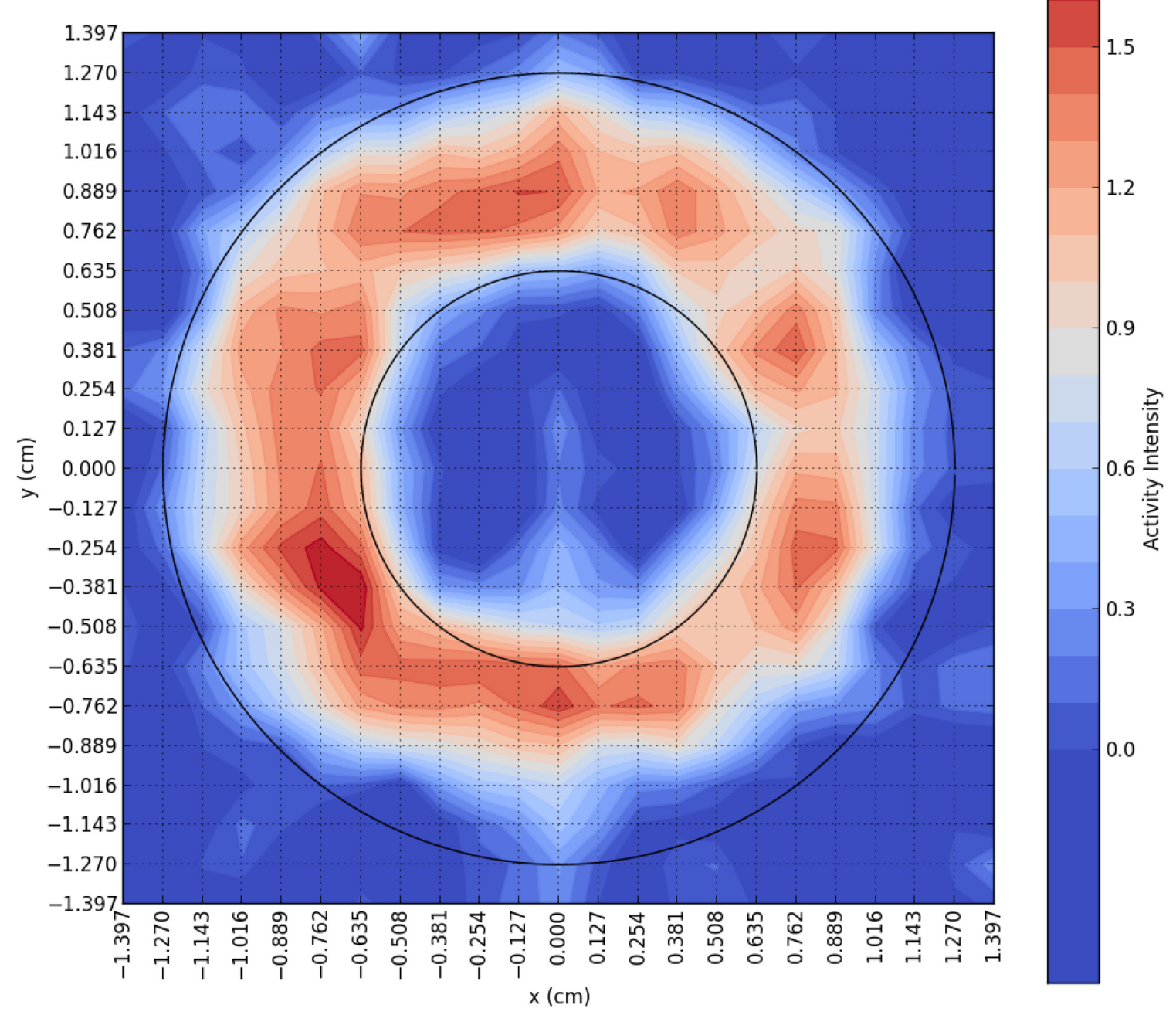


Eu-154

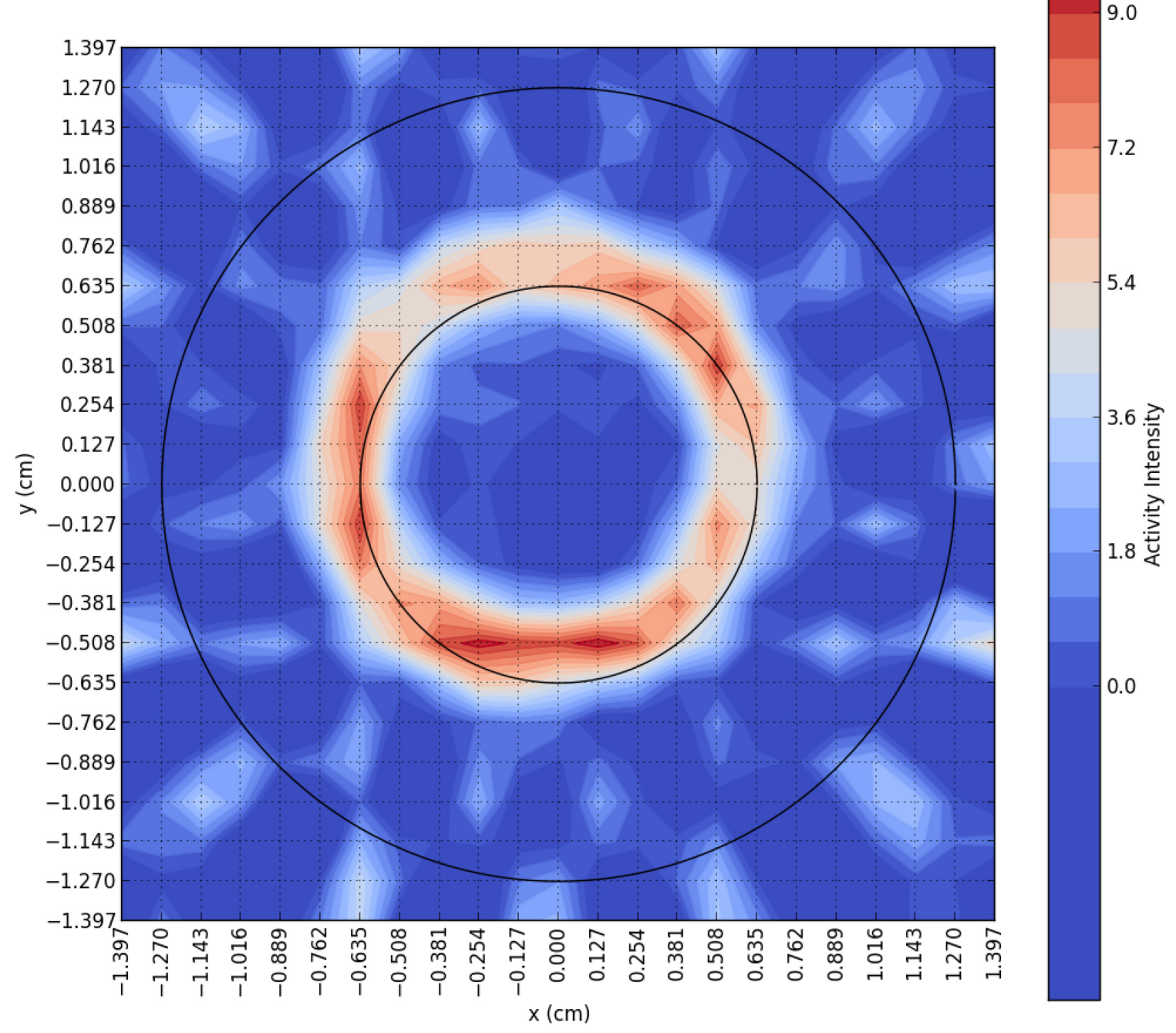




\section{Co-60}

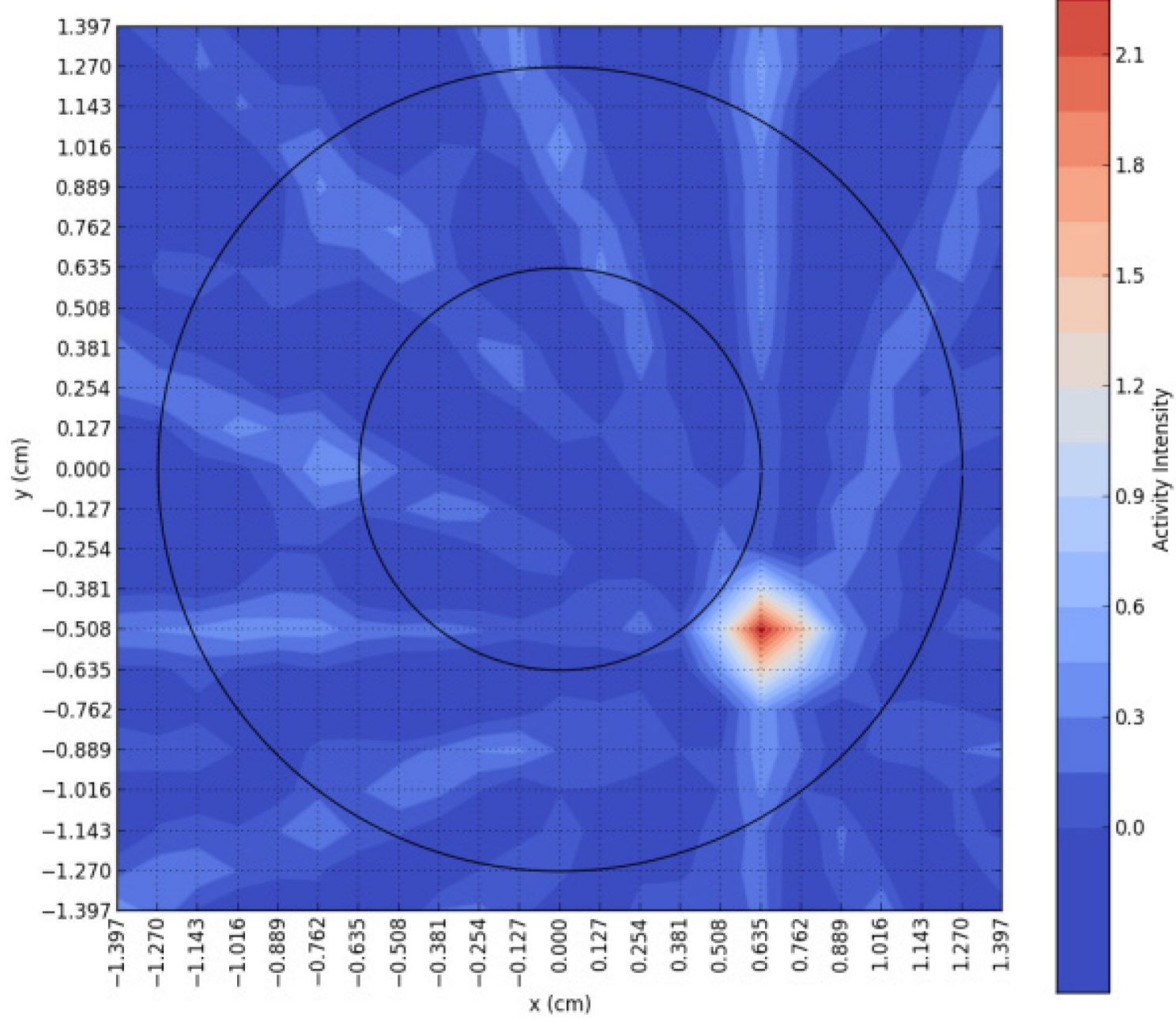




\section{Inner Ring 8 Axial Middle}

Cs-137

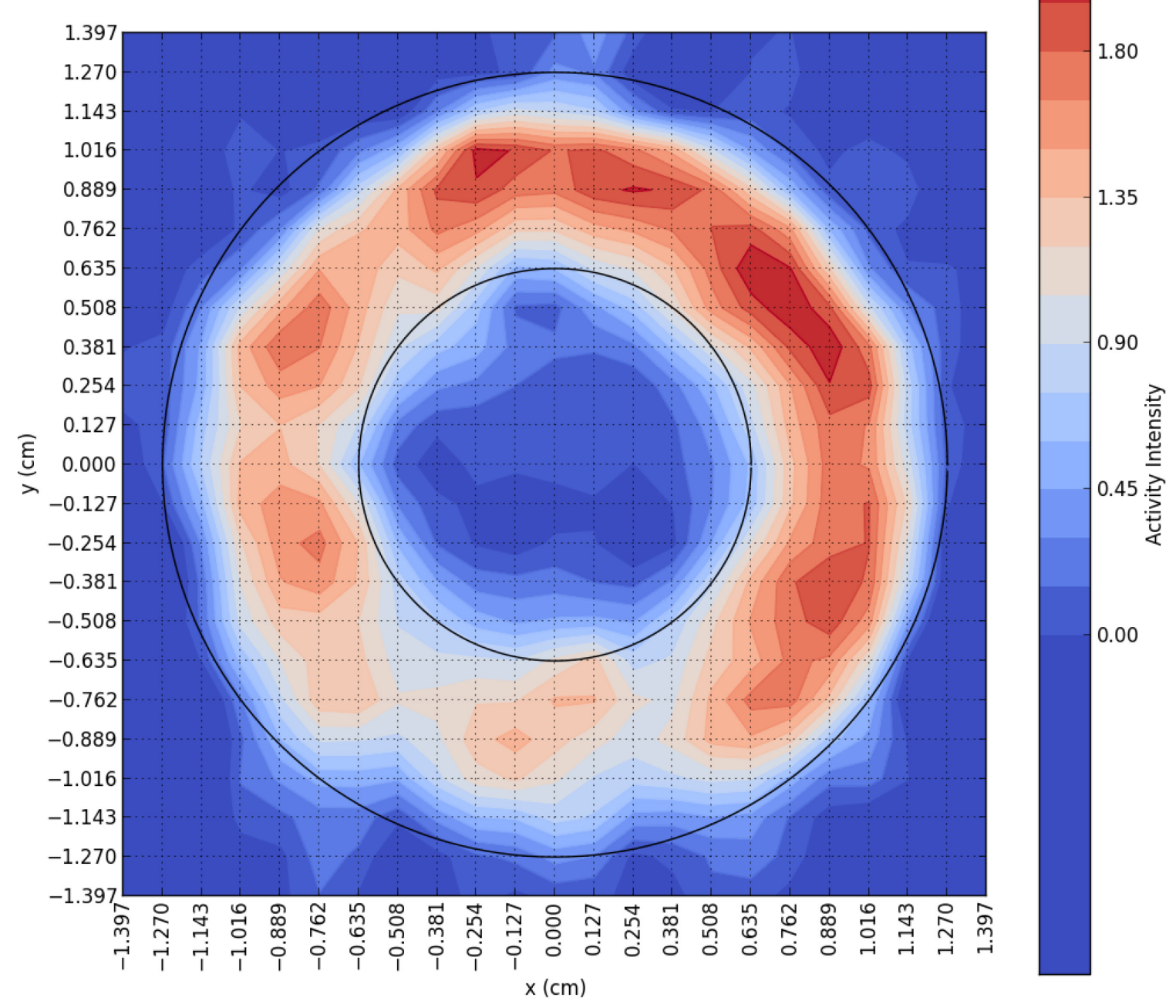


Cs-134

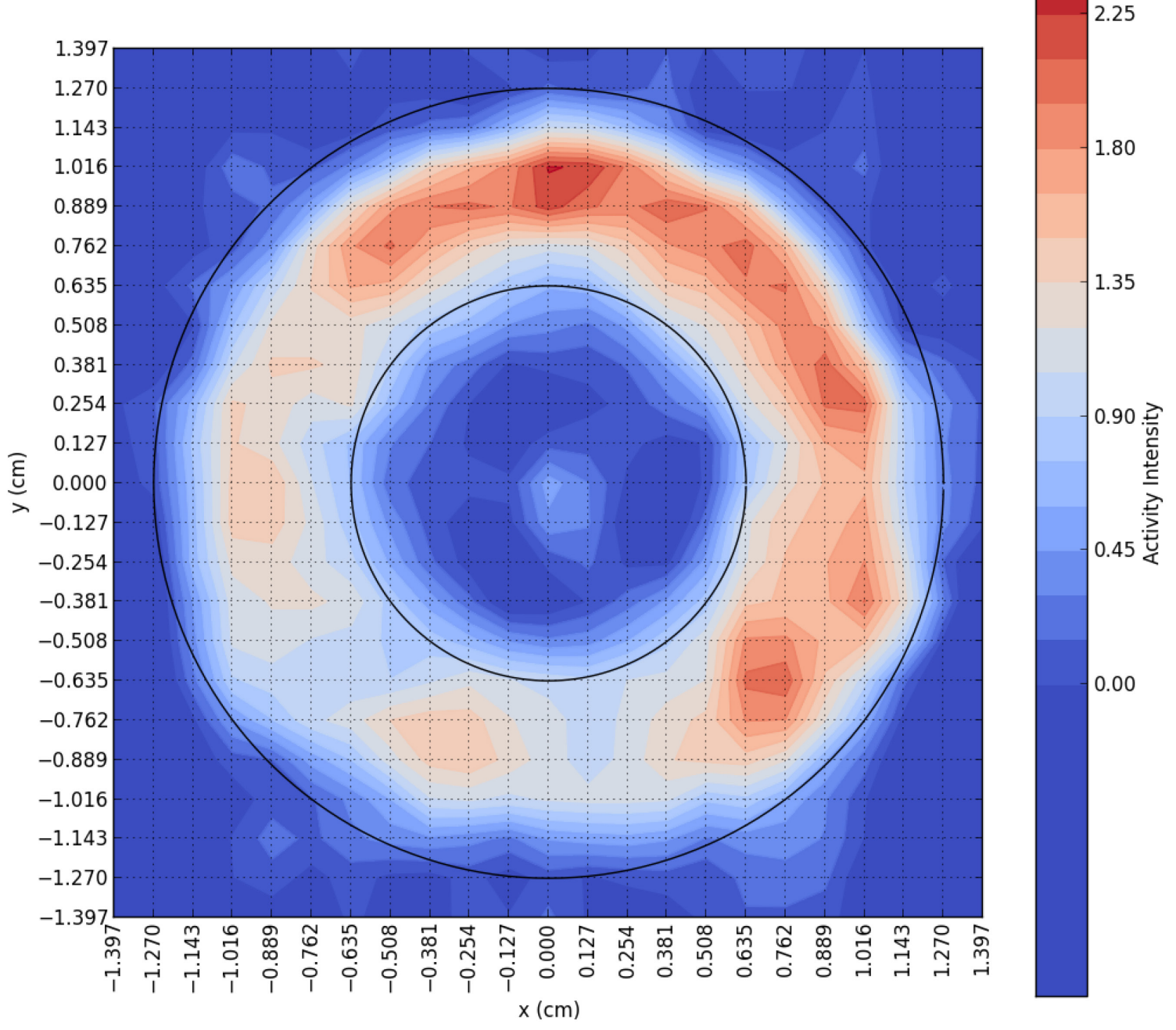




\section{Inner Ring 8 Axial Top}

Cs-137

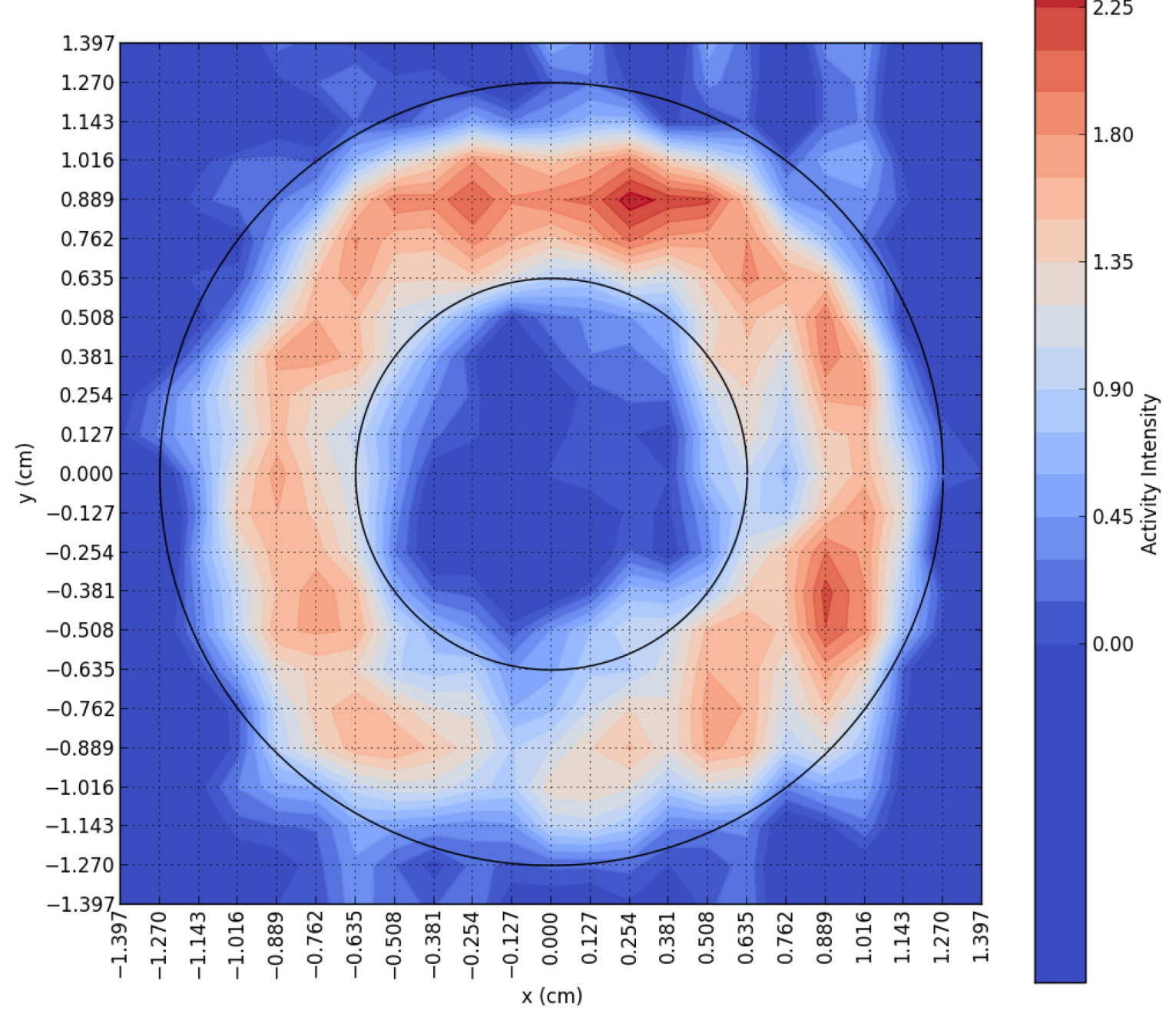


Cs-134

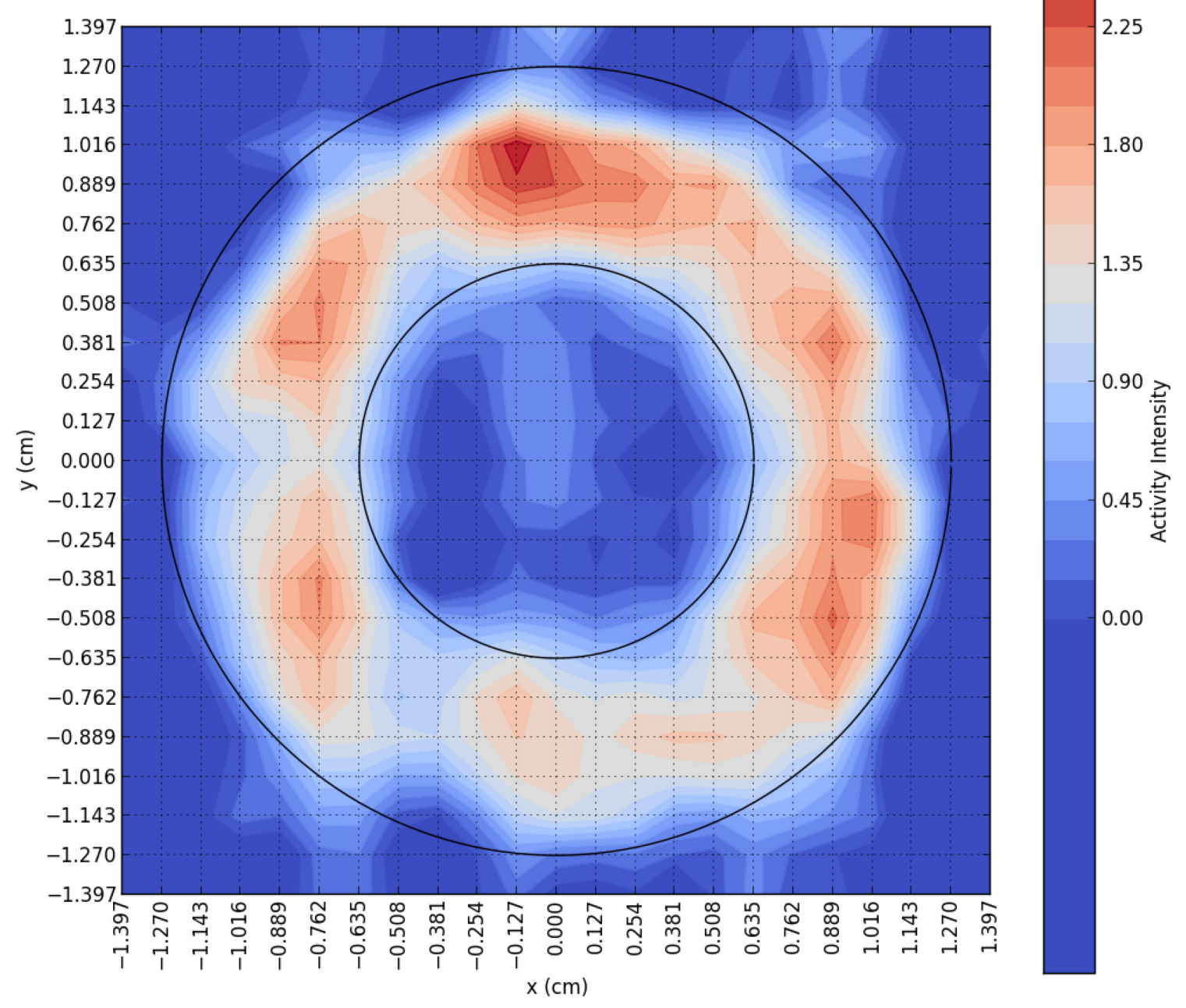




\section{Inner Ring 10 Axial Middle}

Cs-137

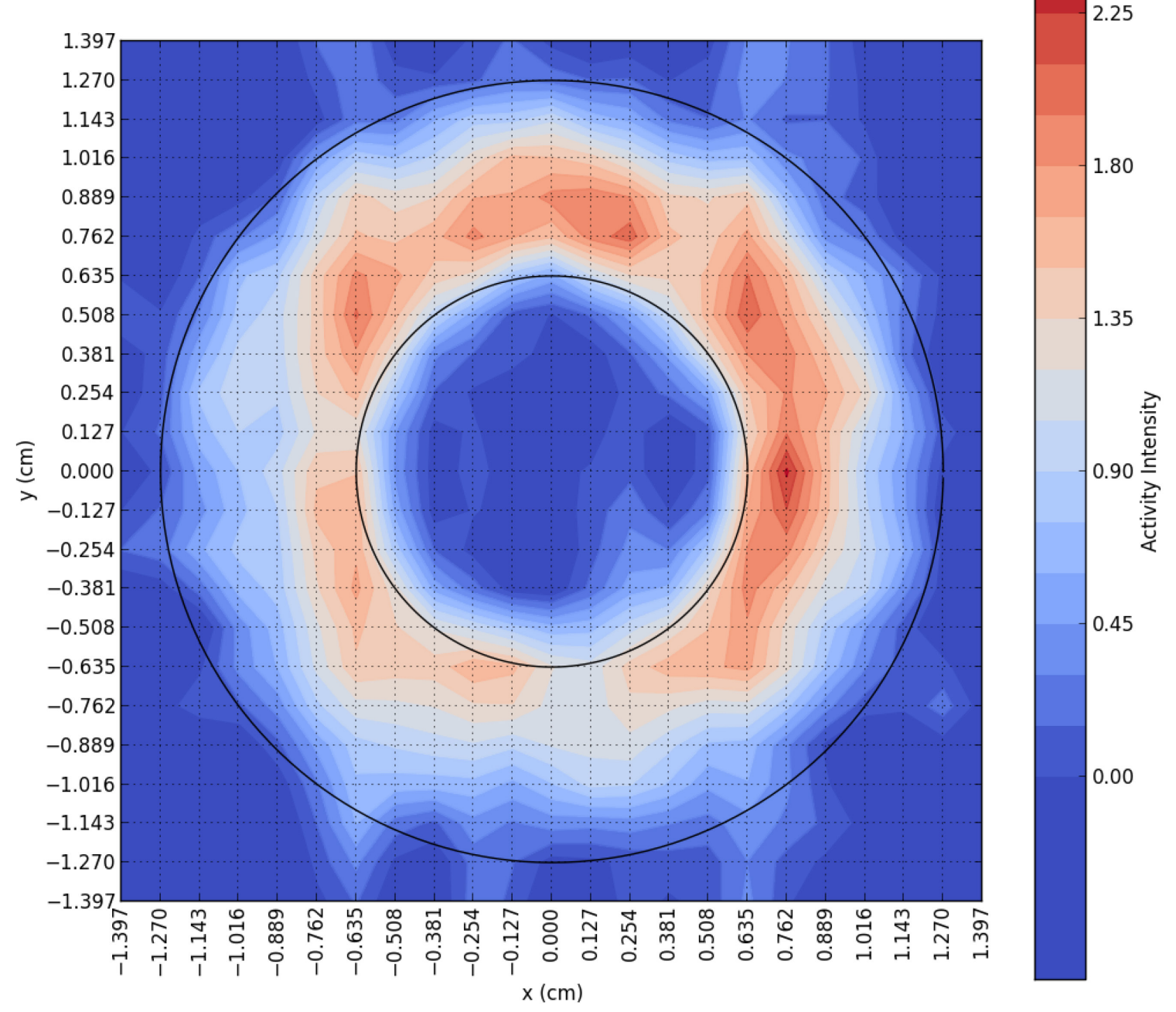


Cs-134

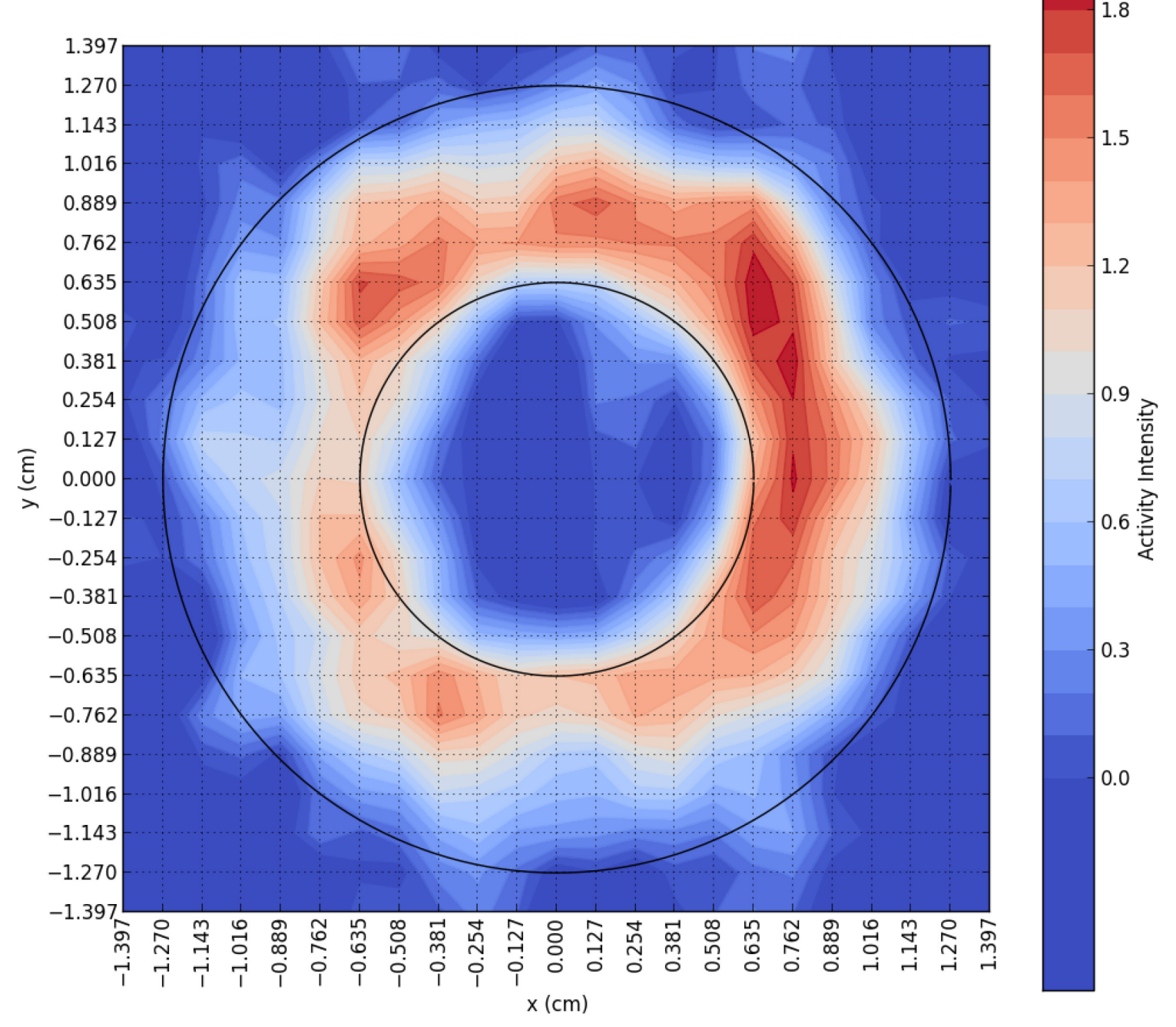


Ag-110m

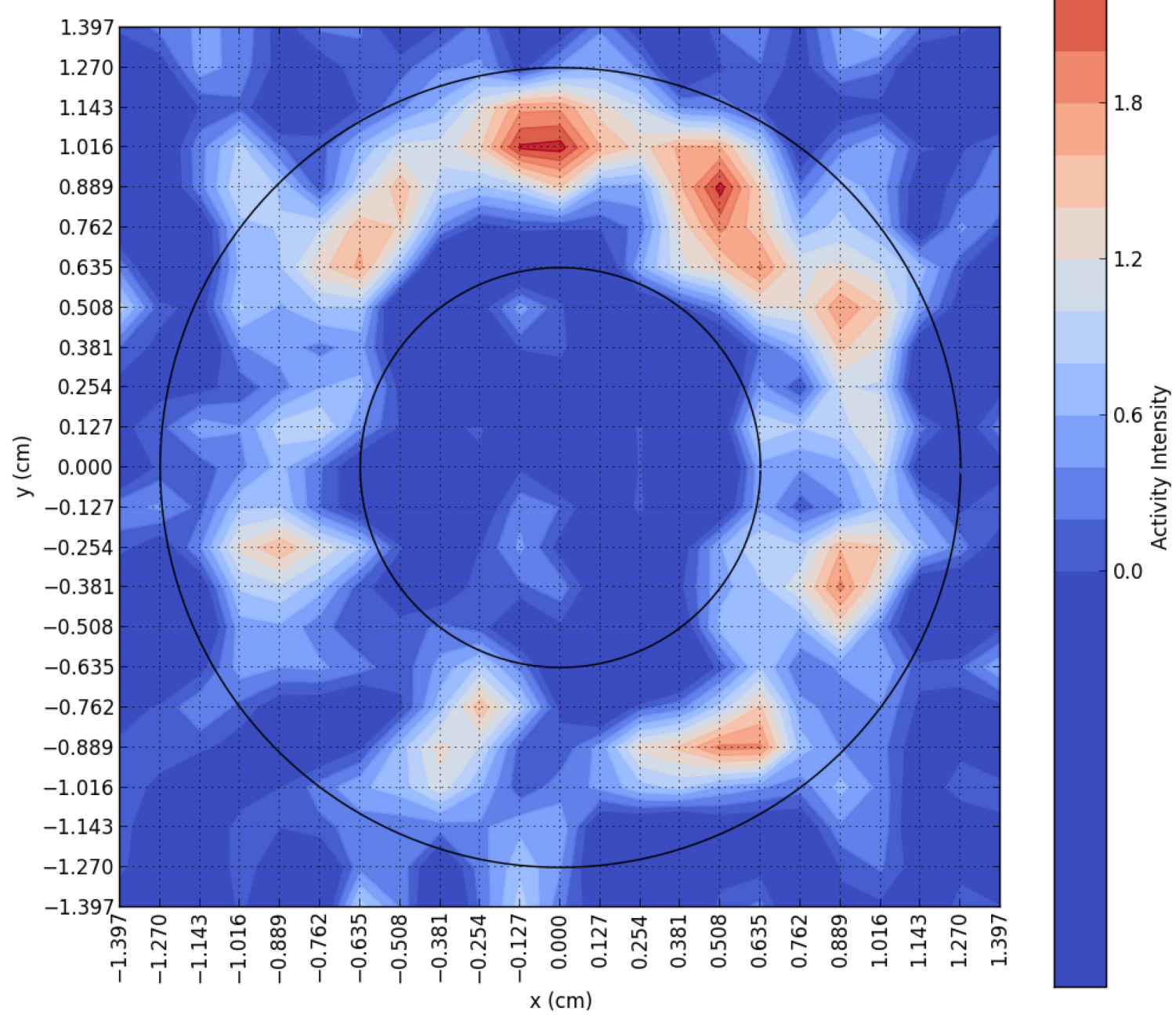




\section{Inner Ring 10 Axial Top}

Cs-137

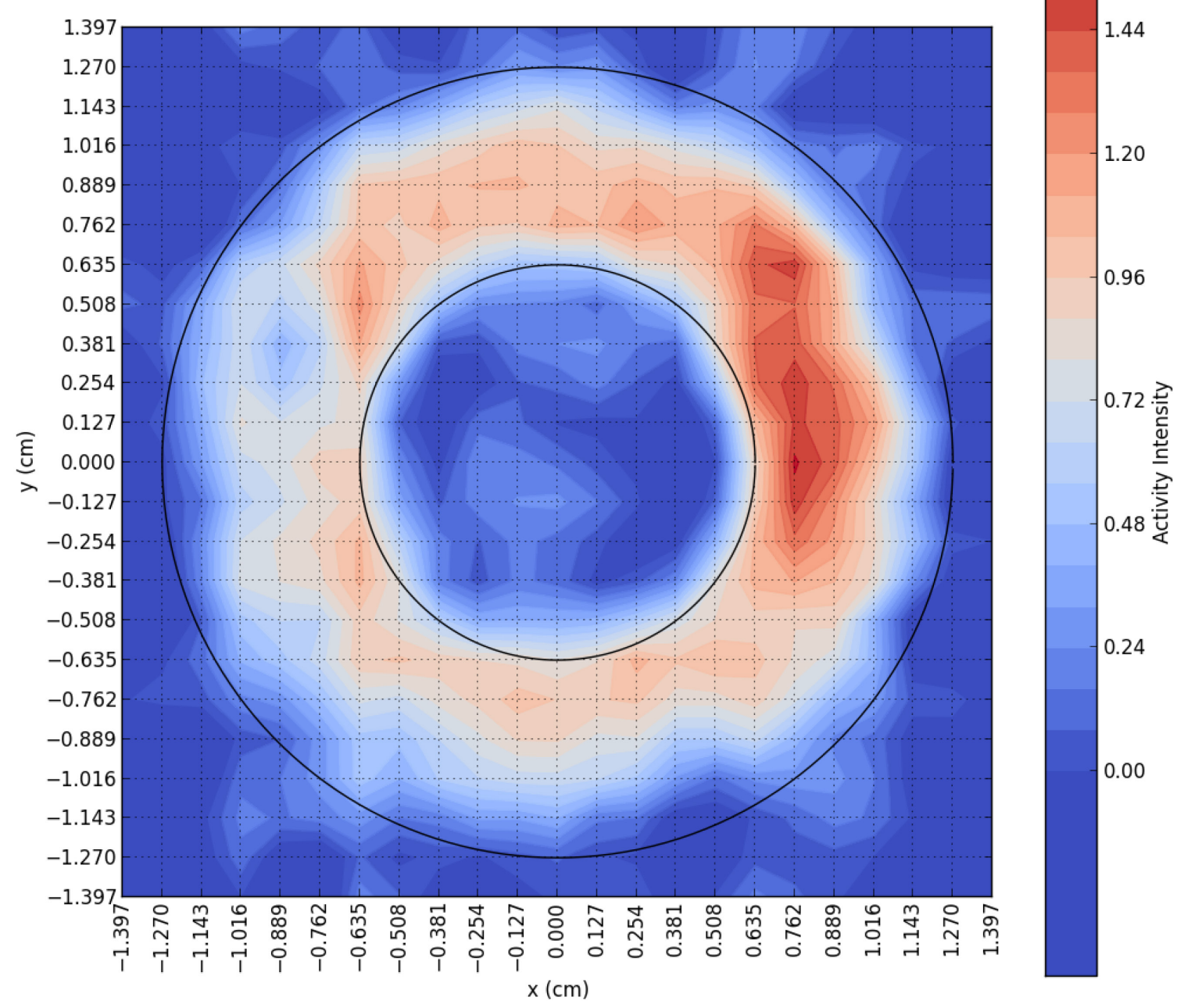


Cs-134

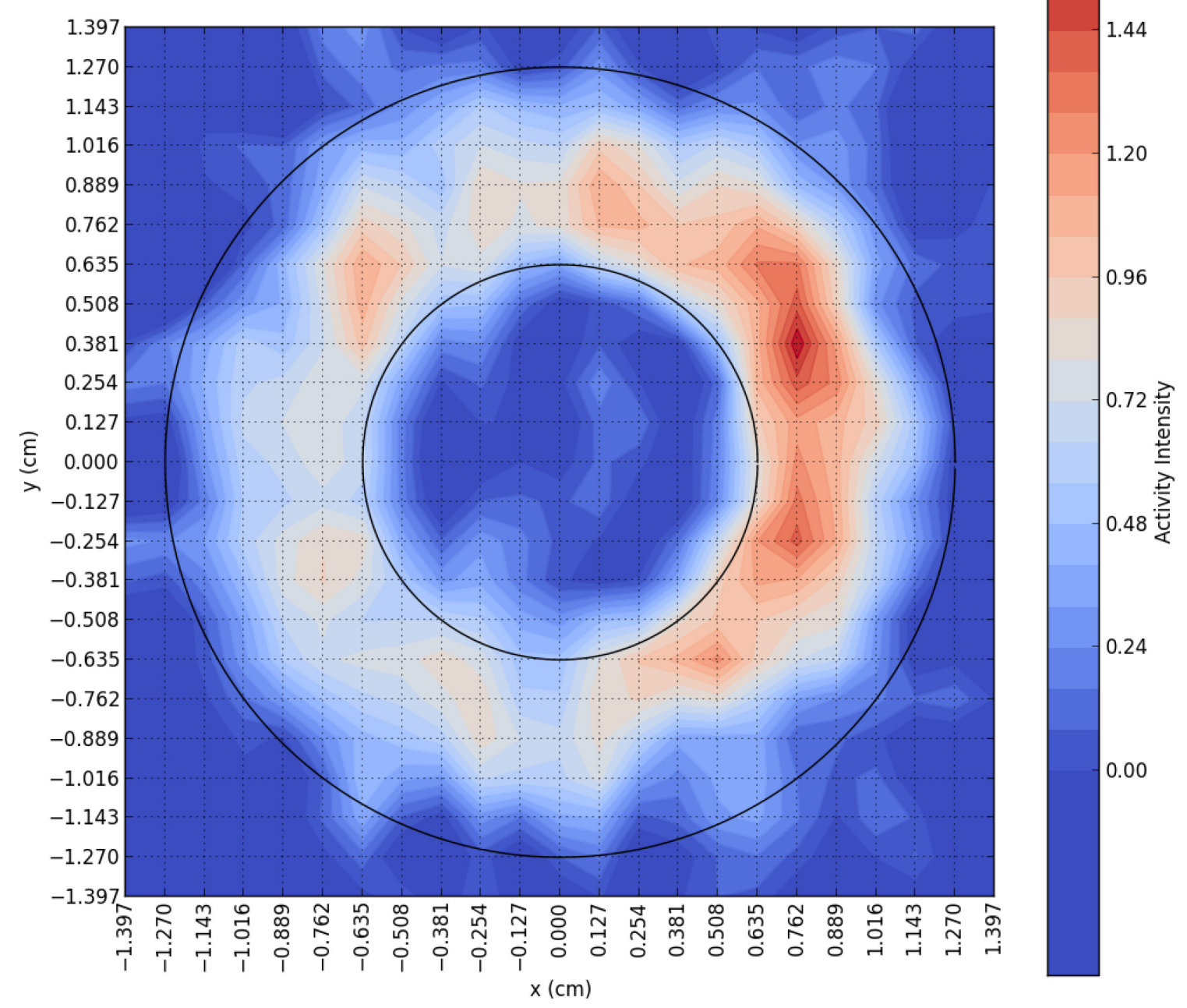


Ag-110m

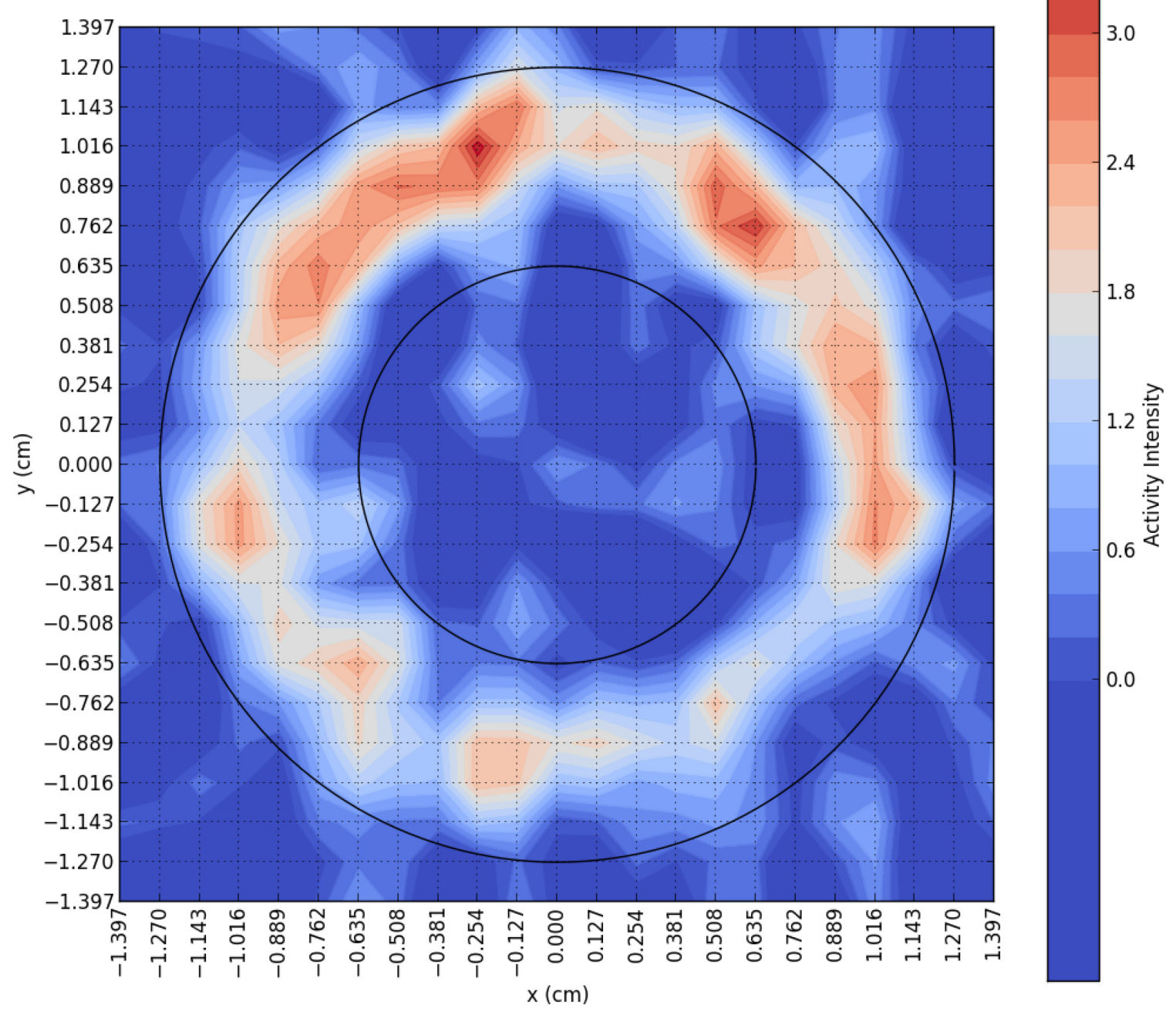




\section{Outer Ring 03 Axial Middle}

Cs-137

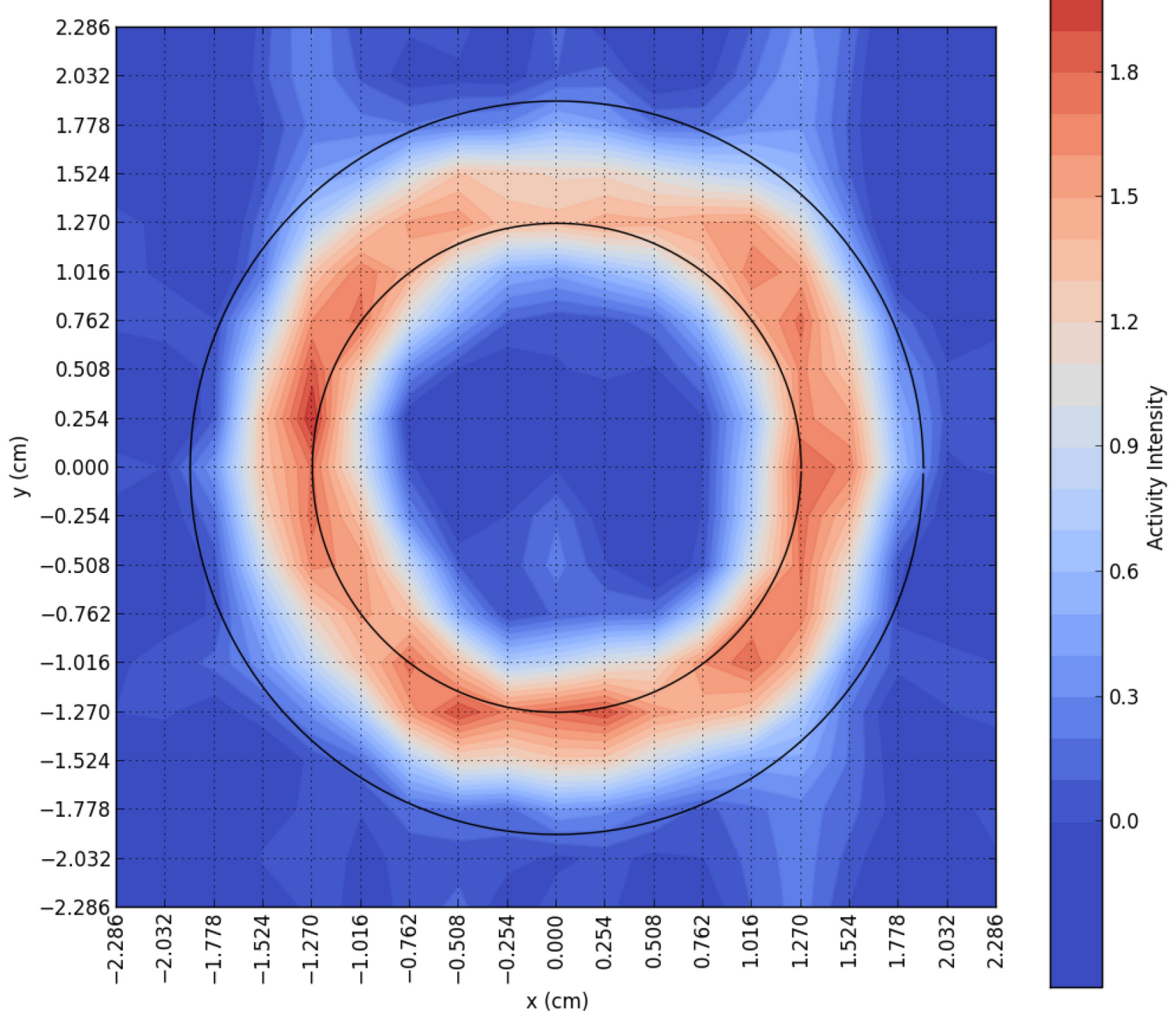




\section{Outer Ring 07 Axial Middle}

Cs-137

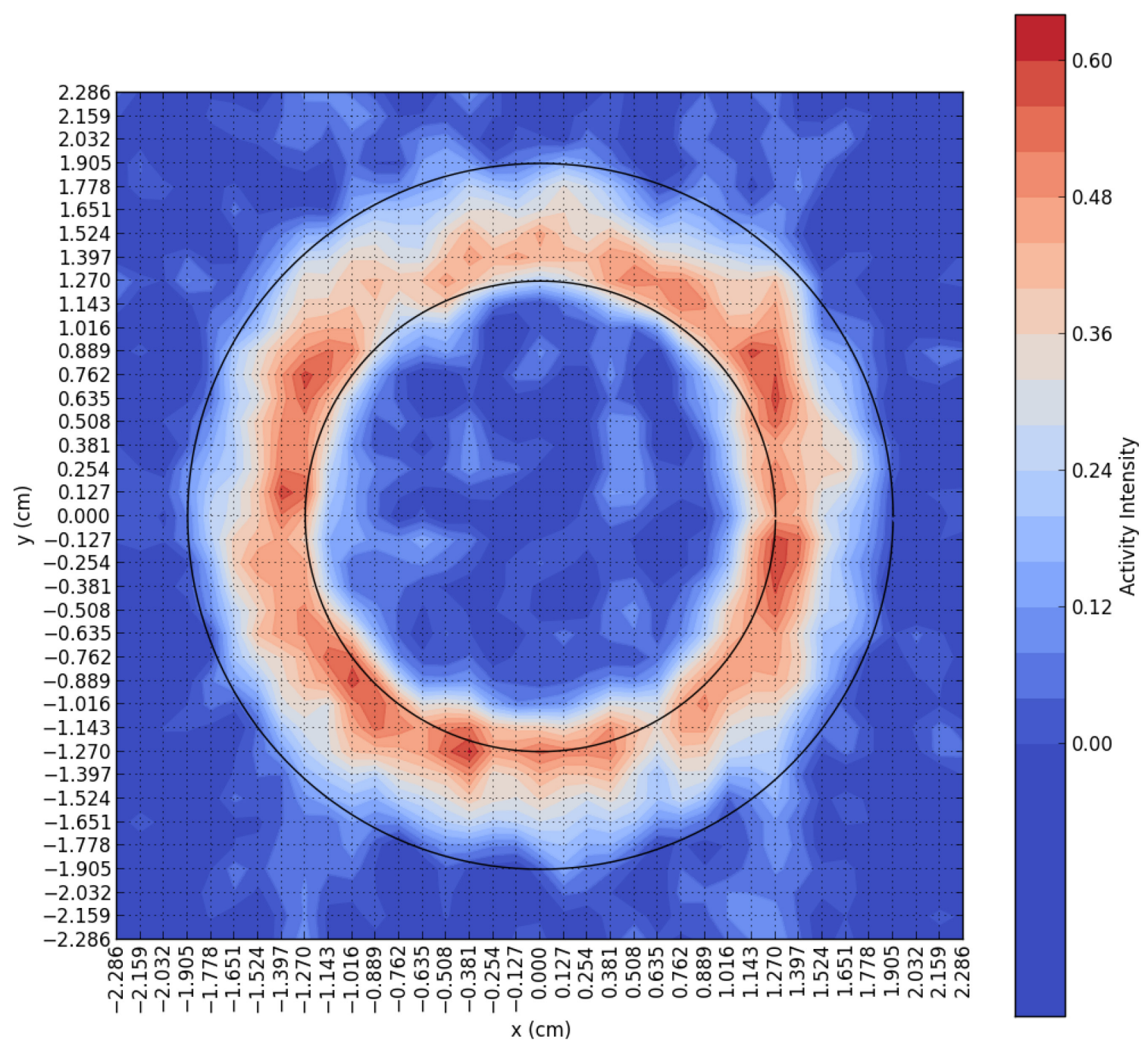


Cs-134

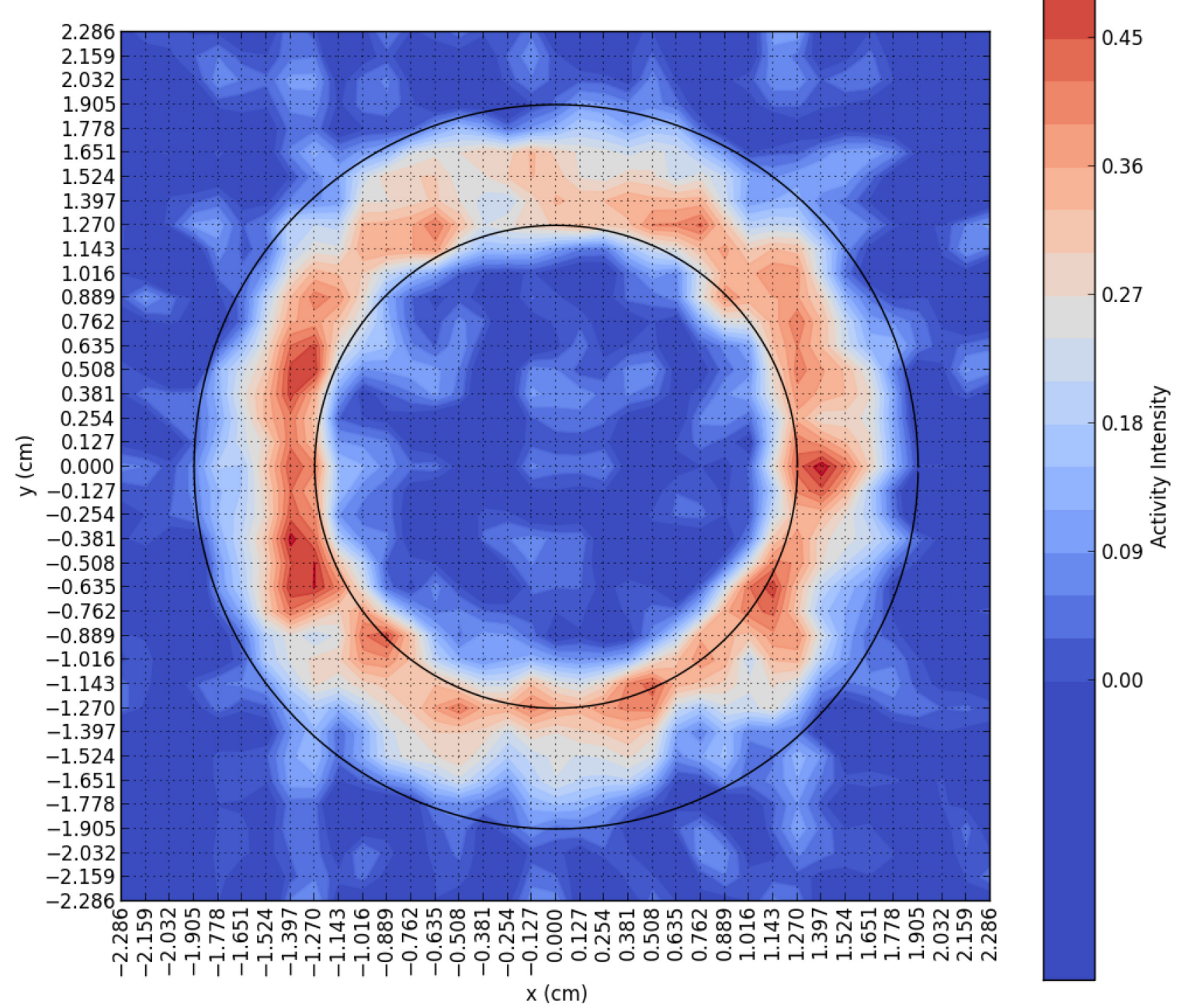




\section{Outer Ring 08 Axial Middle}

Cs- 137

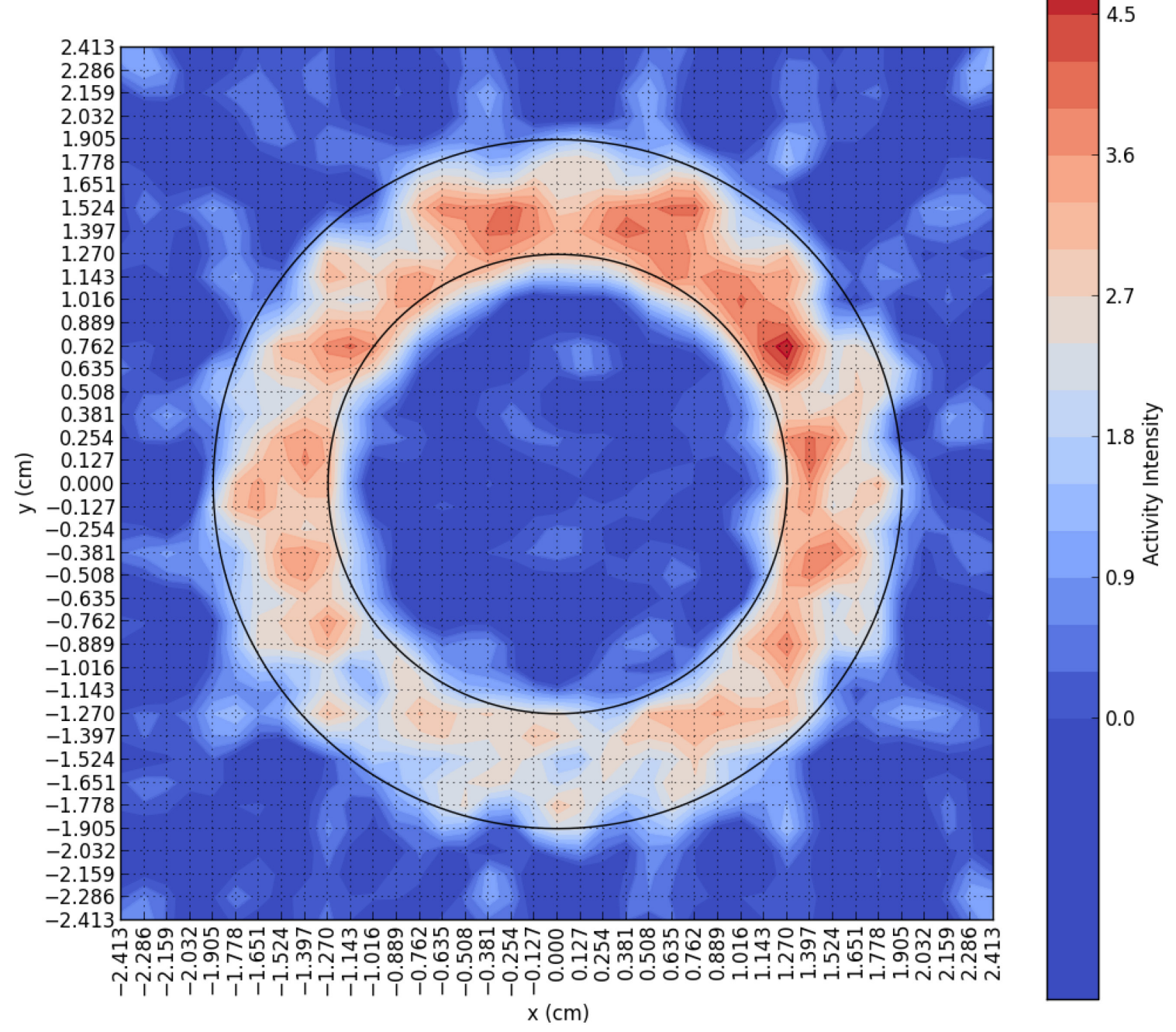


Cs-134

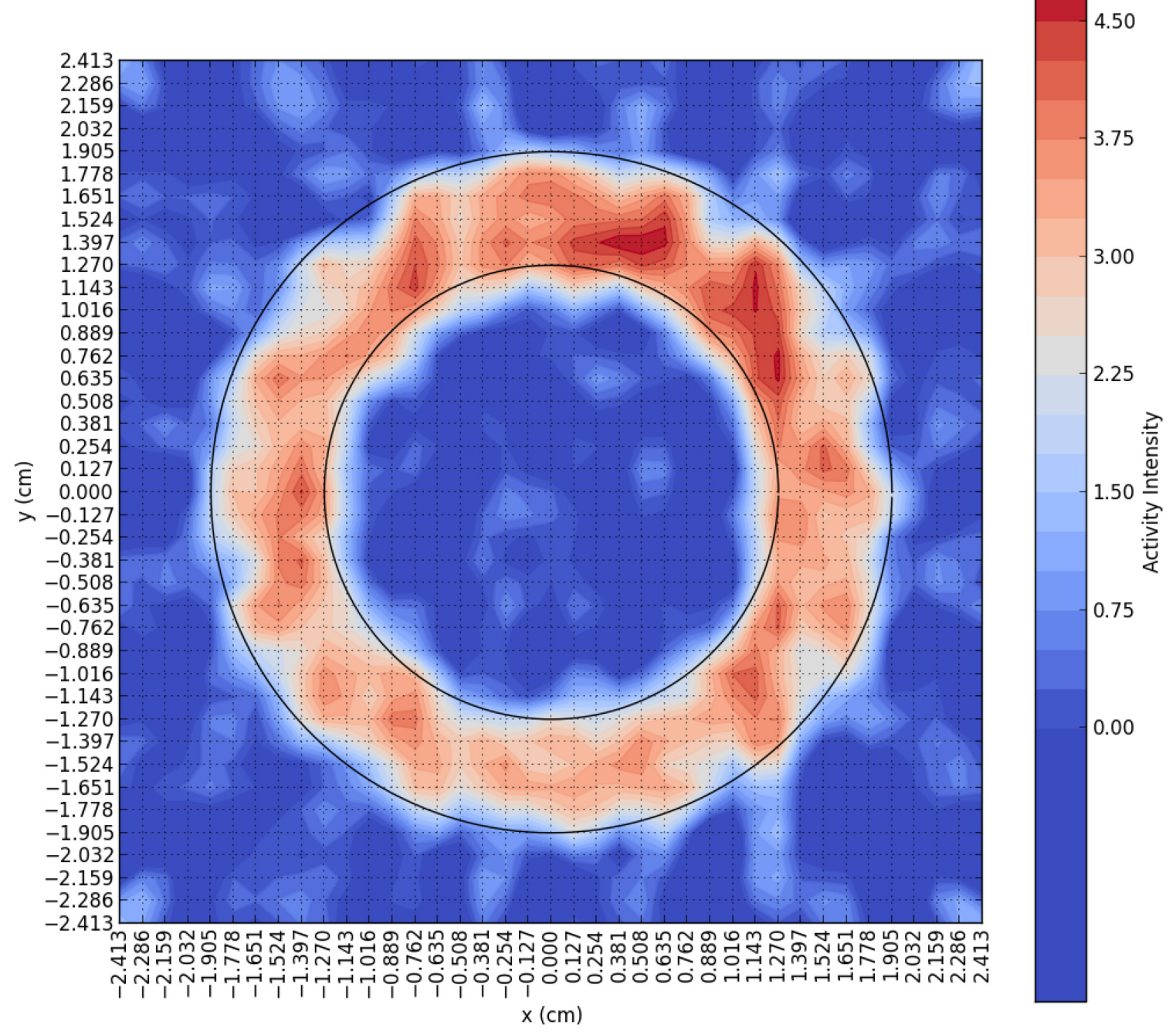


Ag-110m

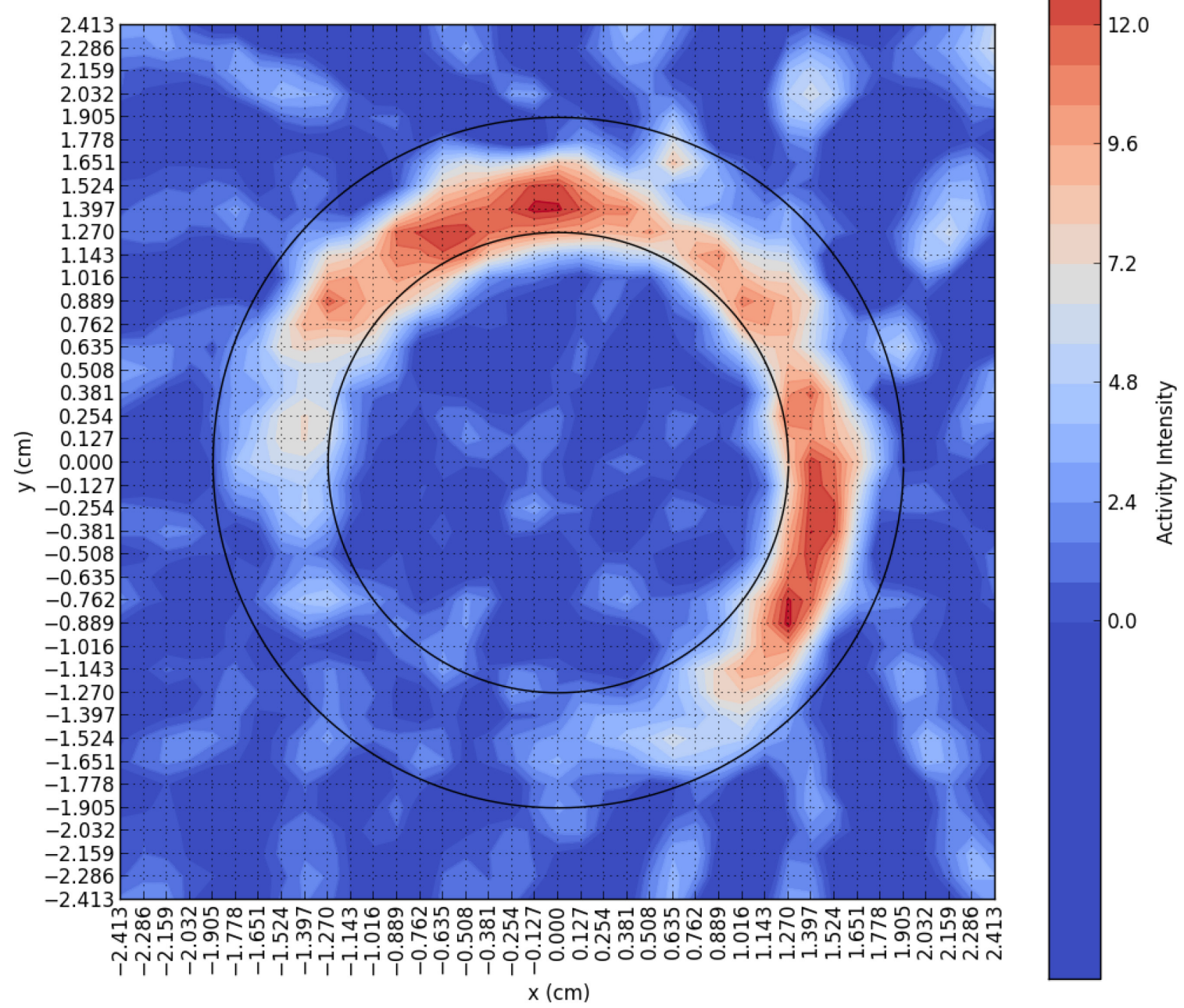

